Florida International University FIU Digital Commons

9-27-2013

\title{
Bionano Electronics: Magneto-Electric Nanoparticles for Drug Delivery, Brain Stimulation and Imaging Applications
}

Rakesh Guduru

rgudu001@fiu.edu

DOI: $10.25148 /$ etd.FI13112201

Follow this and additional works at: https://digitalcommons.fiu.edu/etd

Part of the Bioelectrical and Neuroengineering Commons, Biomaterials Commons, Biomedical

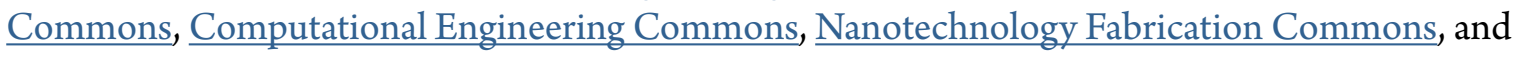
the Other Biomedical Engineering and Bioengineering Commons

\section{Recommended Citation}

Guduru, Rakesh, "Bionano Electronics: Magneto-Electric Nanoparticles for Drug Delivery, Brain Stimulation and Imaging Applications" (2013). FIU Electronic Theses and Dissertations. 979.

https://digitalcommons.fiu.edu/etd/979 


\section{FLORIDA INTERNATIONAL UNIVERSITY}

Miami, Florida

BIONANO ELECTRONICS: MAGNETO-ELECTRIC NANOPARTICLES FOR DRUG DELIVERY, BRAIN STIMULATION AND IMAGING APPLICATIONS

A dissertation submitted in partial fulfillment of the requirements for the degree of DOCTOR OF PHILOSOPHY

in

ELECTRICAL ENGINEERING

by

Rakesh Guduru 
To: Dean Amir Mirmiran

College of Engineering and Computing

This dissertation, written by Rakesh Guduru, and entitled Bionano Electronics: MagnetoElectric Nanoparticles for Drug Delivery, Brain Stimulation and Imaging Applications, having been approved in respect to style and intellectual content, is referred to you for judgment.

We have read this dissertation and recommend that it be approved.

Madhavan Nair

Osama A. Mohammed

Jean H. Andrian

Sakhrat Khizroev, Major Professor

Date of Defense: September 27, 2013

The dissertation of Rakesh Guduru is approved.

Dean Amir Mirmiran

College of Engineering and Computing

Dean Lakshmi N. Reddi

University Graduate School

Florida International University, 2013 


\section{DEDICATION}

I would like to dedicate this dissertation to my family and friends. Their love and support has been the driving force of my life. 


\section{ACKNOWLEDGMENTS}

This dissertation was only possible due to the continuous support from my mentor, family and friends. I would like to specially thank my wife Pallavi Joshi for showing unconditional love and support throughout my Ph.D. I also would like to express my gratitude towards my mentor Dr. Sakhrat Khizroev for providing $24 \mathrm{X} 7$ guidance throughout my research and constantly encouraging me to perform groundbreaking research. I can never imagine completing my Ph.D. without his valuable advises and continuous support. I would also like to thank our collaborator Dr. Madhavan Nair for providing valuable guidance in this cross-disciplinary (Immunology \& Electrical engineering) research. I would further like to extend my thanks to Dr. Jeongmin Hong for helping me in various aspects of my research. 


\begin{abstract}
OF THE DISSERTATION
BIONANO ELECTRONICS: MAGNETO-ELECTRIC NANOPARTICLES FOR

DRUG DELIVERY, BRAIN STIMULATION AND IMAGING APPLICATIONS
\end{abstract}

\author{
by \\ Rakesh Guduru \\ Florida International University, 2013 \\ Professor Sakhrat Khizroev, Major Professor
}

Nanoparticles are often considered as efficient drug delivery vehicles for precisely dispensing the therapeutic payloads specifically to the diseased sites in the patient's body, thereby minimizing the toxic side effects of the payloads on the healthy tissue. However, the fundamental physics that underlies the nanoparticles' intrinsic interaction with the surrounding cells is inadequately elucidated. The ability of the nanoparticles to precisely control the release of its payloads externally (on-demand) without depending on the physiological conditions of the target sites has the potential to enable patient- and disease-specific nanomedicine, also known as Personalized NanoMedicine (PNM). In this dissertation, magneto-electric nanoparticles (MENs) were utilized for the first time to enable important functions, such as (i) field-controlled high-efficacy dissipation-free targeted drug delivery system and on-demand release at the sub-cellular level, (ii) noninvasive energy-efficient stimulation of deep brain tissue at body temperature, and (iii) a high-sensitivity contrasting agent to map the neuronal activity in the brain noninvasively. First, this dissertation specifically focuses on using MENs as energy-efficient and dissipation-free field-controlled nano-vehicle for targeted delivery and on-demand release of a anti-cancer Paclitaxel (Taxol) drug and a anti-HIV AZT 5'-triphosphate 
(AZTTP) drug from 30-nm MENs $\left(\mathrm{CoFe}_{2} \mathrm{O}_{4}-\mathrm{BaTiO}_{3}\right)$ by applying low-energy DC and low-frequency (below $1000 \mathrm{~Hz}$ ) AC fields to separate the functions of delivery and release, respectively. Second, this dissertation focuses on the use of MENs to noninvasively stimulate the deep brain neuronal activity via application of a low energy and low frequency external magnetic field to activate intrinsic electric dipoles at the cellular level through numerical simulations. Third, this dissertation describes the use of MENs to track the neuronal activities in the brain (non-invasively) using a magnetic resonance and a magnetic nanoparticle imaging by monitoring the changes in the magnetization of the MENs surrounding the neuronal tissue under different states.

The potential therapeutic and diagnostic impact of this innovative and novel study is highly significant not only in HIV-AIDS, Cancer, Parkinson's and Alzheimer's disease but also in many CNS and other diseases, where the ability to remotely control targeted drug delivery/release, and diagnostics is the key. 


\section{TABLE OF CONTENTS}

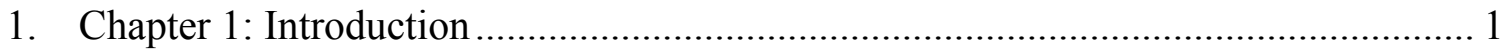

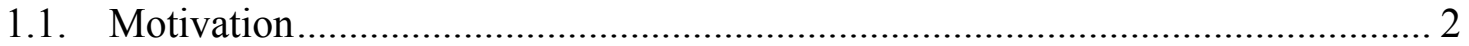

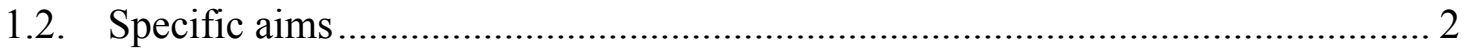

1.3. Nanomaterials for payload delivery, targeted therapy and diagnostics ................ 5

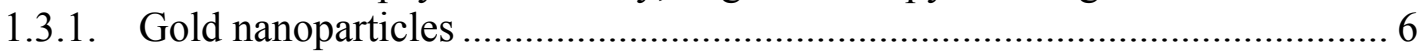

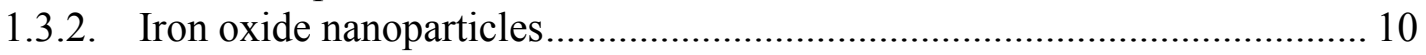

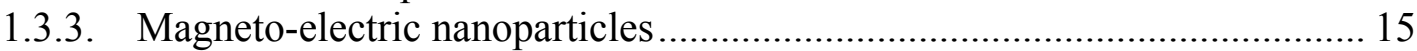

1.4. Artificial brain stimulation for treating neurological disorders ........................... 17

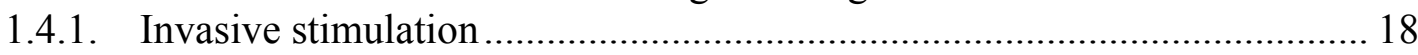

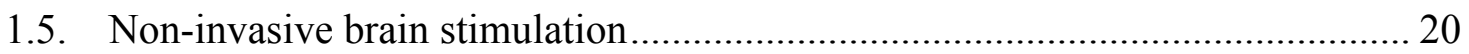

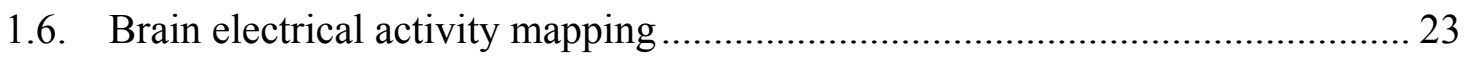

1.6.1. Source of EEG/MEG signal...................................................................... 23

1.7. Unique advantages of magneto-electric nanoparticles over the conventional

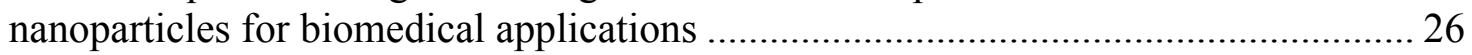

2. Chapter 2: Magneto-electric Nanoparticles for Eradicating HIV from CNS ............ 29

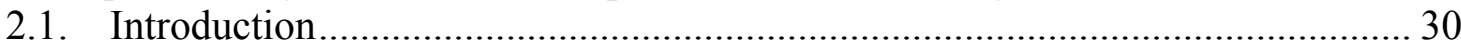

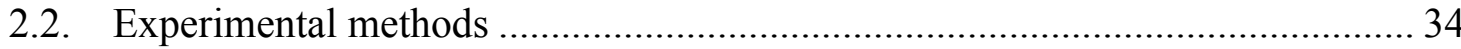

2.2.1. Preparation of CoFe2O4-BaTiO3 coreshell MENs ....................................... 34

2.2.2. Conjugating AZTTP with MENs (AZTTP-MEN Nanoformulations) ......... 35

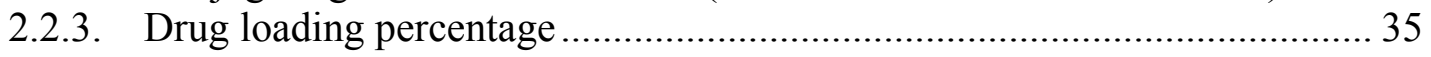

2.2.4. Magnetic Field Treatment....................................................................... 36

2.2.5. Surface Charge of MENs and AZTTP Molecules ...................................... 37

2.2.6. Mass spectrometry analysis of AZTTP molecules, AZTTP bound to MEN before and after the a.c.-triggered release............................................................. 37

2.2.7. Preparation of samples for AFM and FTIR imaging ................................... 39

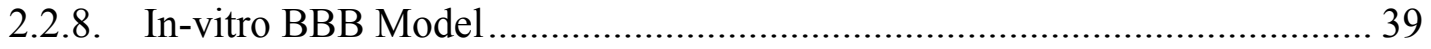

2.2.9. p24 Assay to confirm the integrity of AZTTP drug .................................. 40

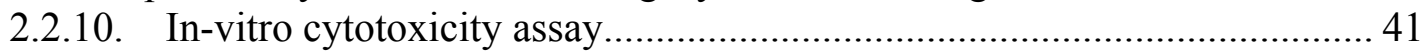

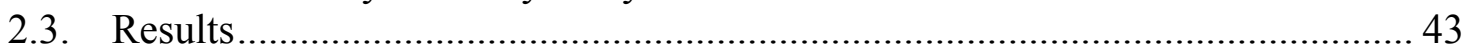

2.3.1. Transmission electron microscopy study of MENs ..................................... 43

2.3.2. Spectrophotometry study of the release ..................................................... 44

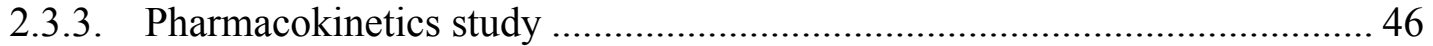

2.3.4. Fourier-transform infra-red analysis ......................................................... 47

2.3.5. Atomic force microscopy study of the release kinetics ............................... 48

2.3.6. Field-controlled delivery and drug release by MENs loaded with AZTTP across BBB in in-vitro model. .......................................................................... 49

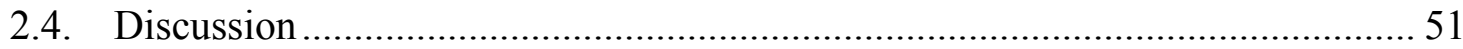

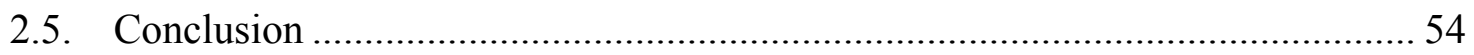


3. Chapter 3: Magneto-electric Nanoparticles to Enable Field Controlled Highspecificity Drug Delivery to Eradicate Ovarian Cancer Cells ........................................ 56

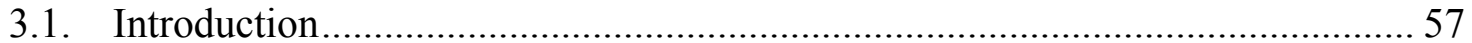

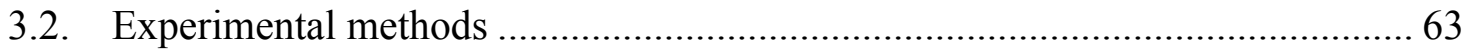

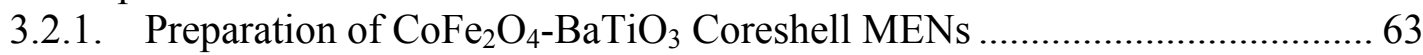

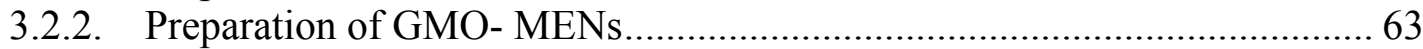

3.2.3. Preparation of HER-2 biomarker antibody conjugated GMO- MENs ........ 64

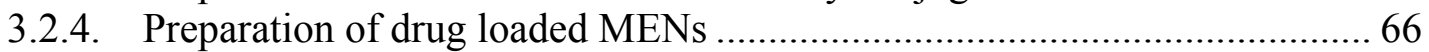

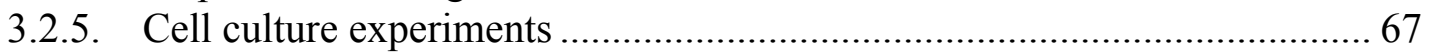

3.2.6. Fluorescence measurements and confocal imaging of drug uptake by SKOV-3 cells for different drug-carrier combinations .............................................. 68

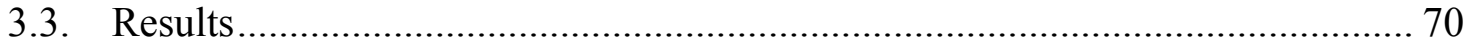

3.3.1. Field-controlled drug release by MEN-based carriers ................................. 71

3.3.2. Field-controlled drug uptake by tumor cells through the MEN-induced nano-

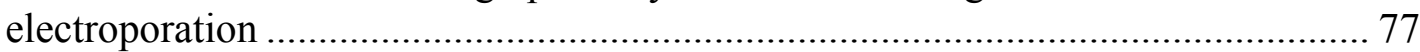

3.3.3. Confocal microscopy to visualize the internal drug localization in SKOV-3

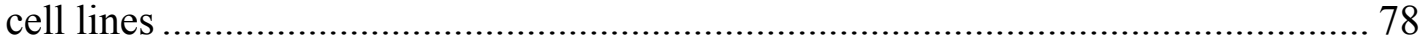

3.3.4. Field dependence of drug uptake in cancer and healthy cells...................... 78

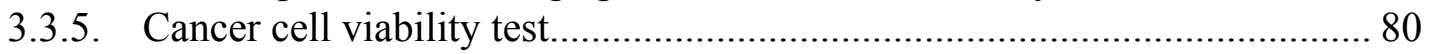

3.3.6. In-vitro Cytotoxicity Assay ....................................................................... 82

3.3.7. Heat-dissipation due to field-treatment with MENs ................................... 83

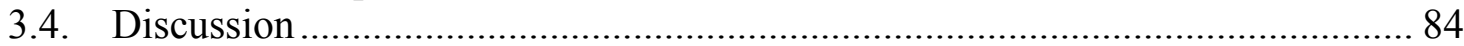

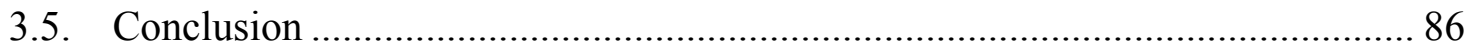

4. Chapter 4: Functionalized Magneto-electric Nanoparticles for Controlling the Drugreleasing Threshold Field........................................................................................ 88

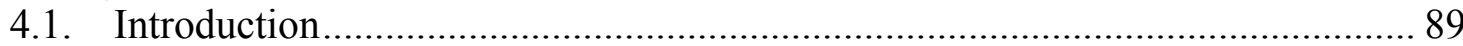

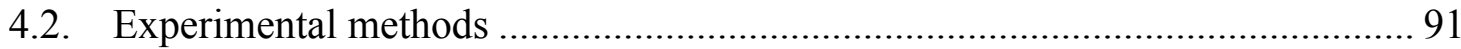

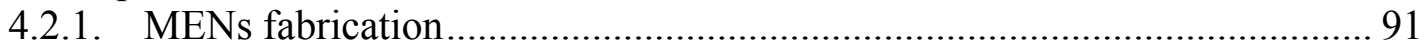

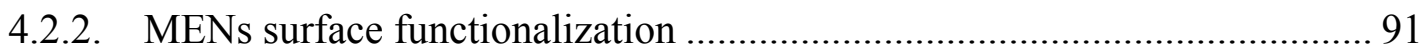

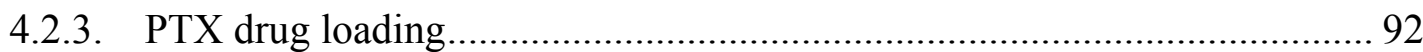

4.2.4. Remote magnetic field triggered PTX drug release..................................... 93

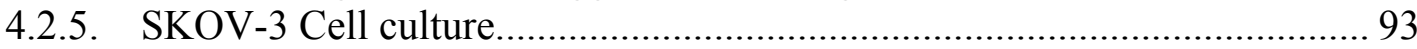

4.2.6. In-vitro Cytotoxicity measurements ............................................................. 94

4.2.7. MENs size and surface charge measurements ........................................... 94

4.2.8. Sample preparation for FT-IR measurements............................................. 95

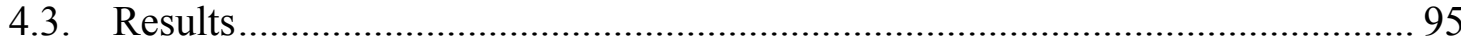

4.3.1. MEN's Intermediate (Functionalization) Layer........................................ 95

4.3.2. PTX Drug Loading onto MENs surface ..................................................... 98

4.3.3. On-Demand Paclitaxel Release Using Remote Magnetic Fields................. 99

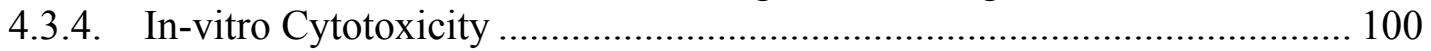

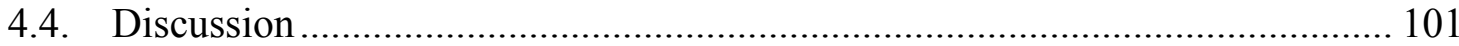

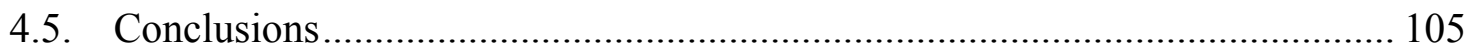


5. Chapter 5:Magneto-electric Nanoparticles for Non-invasive Brain Stimulation .... 107

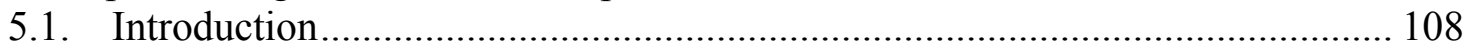

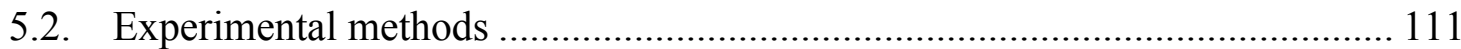

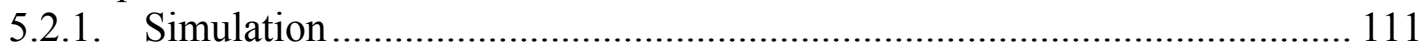

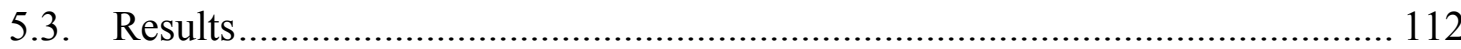

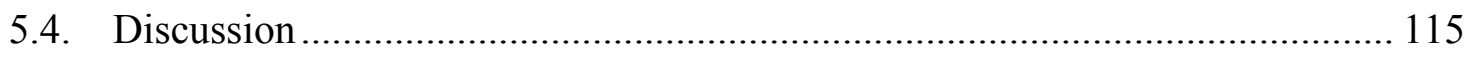

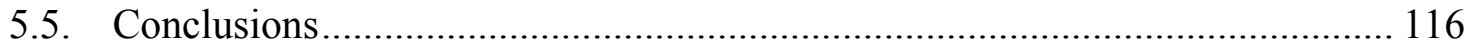

6. Chapter 6: Brain Activity Mapping Using Magneto-electric Nanoparticles Non-

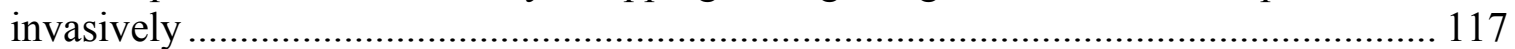

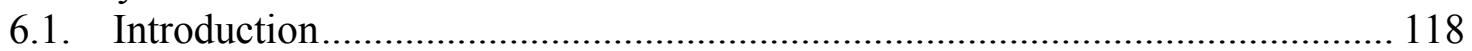

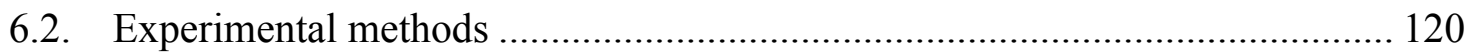

6.2.1. Simulation of relative magnetization of nanoparticles .............................. 120

6.2.2. MRI and MNI signal intensity simulations........................................... 122

6.2.3. Brain segmentation of the MRI data ..................................................... 123

6.2.4. Tissue properties ................................................................................ 123

6.2.5. Tracking the neuronal activity of the brain.............................................. 123

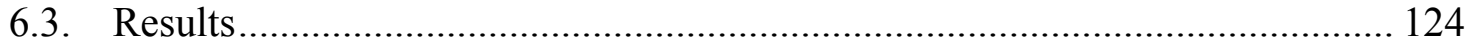

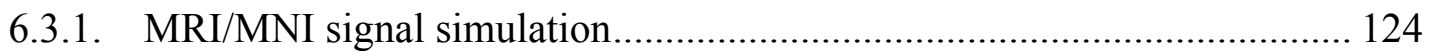

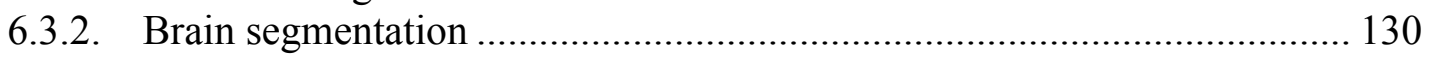

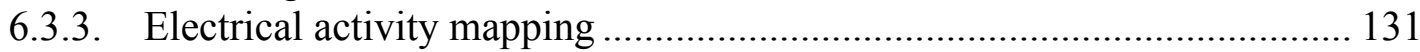

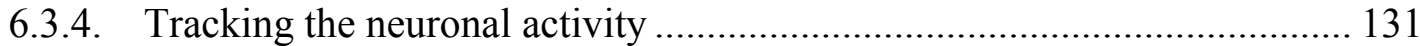

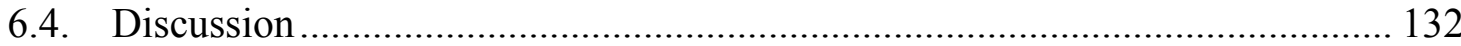

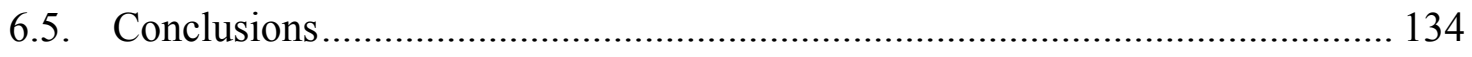

7. Chapter 7: Conclusions and Future Work ………………………………............ 135

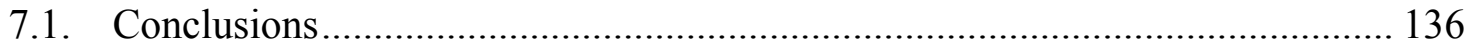

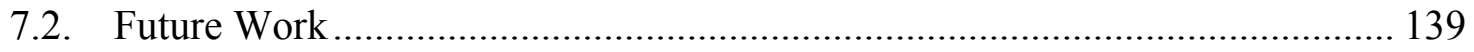

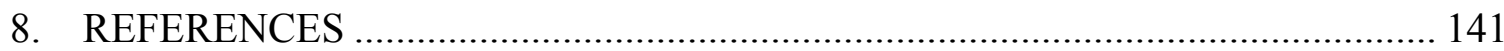

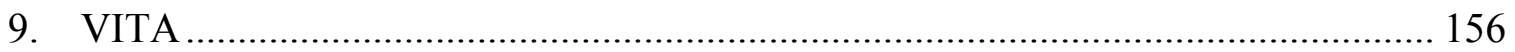




\section{LIST OF FIGURES}

FIGURE

PAGE

Figure 1: A) AuNPs for delivering the therapeutic agents. a) bare-AuNPs, b) AuNPs loaded with therapeutic agents (proteins/DNA/RNA/drugs), c) encapsulation of AuNPs loaded with therapeutic agents, and d) release of therapeutic agents by the physiological ( $\mathrm{pH}$ and temperature) and external stimulations (light). Figure 1B, a) cancer cell surface with biomarkers, b) AuNPs, tagged with anti-biomarkers, c) accumulations of biomarker tagged-AuNPs on cancer cell surface, and d) a surface plasmon resonant absorption spectroscopy image showing only cancer cells through AuNPs accumulation. 7

Figure 2: Advantages and disadvantages of AuNPs in various biomedical applications. 10

Figure 3: shows advantages and disadvantages of using magnetic nanoparticles for various applications. 14

Figure 4: The relationship between the magneto-electric and multiferroic materials..... 16

Figure 5: TMS and tDCS principles of non-invasive brain stimulation. 21

Figure 6: Signal in EEG/MEG. The synchronous firing of the neurons with the electrical currents and the associated magnetic fields are illustrated in the image. 25

Figure 7: Illustration of the underlying physics of the a.c.-field-triggered release. A simplified (one-directional) illustration of the concept of on-demand drug (AZTTP) release by MENs stimulated by a uniform a.c. magnetic field in X-direction: (a) At zero field, only the ionic charge is present in the MEN shell; (b) An additional dipole moment (proportional to the magnetic field) breaks the original symmetry of the charge distribution in the shell; (c) As the field is increased above the threshold value $\left(\sigma_{\text {ionic }}\right.$ $\left.\sigma_{\mathrm{ME}}\right)$, the bond on one side is broken. (d) and (e) The field is reversed to break the bond on the opposite side of the nanoparticle. The red arrows show the electric dipole due to the $\mathrm{ME}$ effect. In practice, due to the random configurations of nanoformulations with respect to the field, the effect is present along every central bond orientation................. 33

Figure 8: Magnetic fields treatment setup. Drug loaded MENs are treated in the uniform magnetic fields using a pair of Helmholtz coils

Figure 9: Mass spectrometry analysis: the number of counts versus the mass-to-charge ratio for (top) the free AZTTP, (middle) AZTTP and MENs in the bound state, (bottom) AZTTP and MEN after the a.c. release. 38

Figure 10: P24 study to prove that the on-demand release does not affect the inhibition efficacy of the AZZTP drug. The P24 concentration is in $\mathrm{pg} / \mathrm{ml}$ (units). 40 
Figure 11: XTT assay optimization assay results for human astrocyte cells for different treatment times $(2,4$, and $6 \mathrm{hrs}$.).

Figure 12: Transmission electron microscopy analysis of MENs. (a) A transmission electron microscopy (TEM) image of MENs. The core-shell structure of MEN is highlighted. The scale bar is $100 \mathrm{~nm}$. (b) An energy-dispersive spectroscopy (EDS) analysis of MENs.

Figure 13: Field strength and frequency dependence of the drug release. Chart showing the release efficacy of AZTTP drug bound to MENs by a remote magnetic field at different amplitudes and frequencies. 45

Figure 14: Pharmacokinetics study. Pharmacokinetics Study: 3-D Chart representation of the drug release percentage at various combinations of the field strength $(12,44$, and 66 Oe), the frequency $(0,100$, and $1000 \mathrm{~Hz})$, and the treatment duration $(1,5,10,60$, and $120 \mathrm{~min}$ ). 46

Figure 15: FTIR measurements. FTIR measurements for MEN-AZTTP system at three different stages: (i) (top) MENs only, (ii) AZTTP-loaded MENs, and (iii) MENs after the release by the a.c.-field treatment. 48

Figure 16: Atomic force microscopy study of the drug release kinetics. AFM images at different stages of the release process: (a) MENs and (b) AZTTP chains before the loading (binding) step, (c) AZTTP-MEN nanoformulations after the loading process, (d) MENs and (e) AZTTP after the drug release by a 44-Oe a.c. field at $1000 \mathrm{~Hz}$. The scale bar is $100 \mathrm{~nm}$. 49

Figure 17: AZTTP-MEN translocation of across BBB. 50

Figure 18: Hypothesis illustration: MENs as field-controlled nano-electroporation sites to let the drug through the cancer cell membranes. An artist's view that illustrates how the electric medium in the cell membrane in the vicinity of MENs can effectively act as a field-controlled array of nanoscale localized valves for the drug-loaded MENs to enter the cell. The "valves" are open as the remote magnetic field is above the first critical value, $H_{\text {th }}$, specific to the cell. This value for the tumor cell is at least a factor of two lower than that for the healthy cell. As the field is further increased above the second critical field, $\mathrm{H}_{\mathrm{r}}$, the release is initiated. 59

Figure 19: Energy dispersion spectroscopy of MENs. Energy dispersion spectroscopy (EDS) results depict the composition of (a) MENs only and (b) GMO-coated MENs.... 64

Figure 20: Standard HER-2 antibody concentration calibration curve. Standard HER-2 Antibody plot was obtained using Bio-Rad protein assay kit at $595 \mathrm{~nm}$ absorbance....... 66

Figure 21: PTX drug loading percentages. PTX drug loading percentage for MENs, GMO-MENs and HER-2-GMO-MENs after 3 hrs. incubation ( $\mathrm{n}=3$ ). 67 
Figure 22: PTX calibration plot. The absorption maxima and the standard linear calibration plot for PTX at different drug concentration values determined spectrophotometrically.

Figure 23: Photo-absorption measurements of the release kinetics. (A) PTX drug release form GMO-MENs, when treated at varying magnetic field strengths and frequencies after 1-minute treatment $(n=3)$. (B) 3-D Chart that illustrates the kinetics of the drug release. The data were measured spectrophotometrically as the absorbance at $230 \mathrm{~nm}$ wavelength.

Figure 24: Atomic force microscopy. Topography and phase-contrast atomic force microscopy (AFM) images of (A and B) GMO-MENs only, (C and D) GMO-MENs loaded with PTX, (E and F) GMO-MENs after the 36-Oe 1000-Hz field-triggered release, and $(\mathrm{G}$ and $\mathrm{H})$ PTX only. The imaging was performed in Tapping Mode ${ }^{\mathrm{TM}}$ using Nanoscope IIIa Multimode AFM (Veeco Metrology, CA). The samples were specially prepared for the imaging by dispersing desired MENs on the silicon wafer surface $(1 \times 1$ $\mathrm{cm}^{2}$ ). To achieve uniform dispersion of MENs, the nanoparticles of choice were first mixed in chloroform solution ( $1 \mathrm{mg}$ of particles in $600 \mu \mathrm{l}$ of chloroform). Then, $100 \mu 1$ of this solution was mixed with $200 \mu \mathrm{l}$ of isopropanol and the solution was set to 3500-rpm centrifugation for 3 minutes. Finally, the obtained pellet of MENs was dissolved in 200 $\mu \mathrm{l}$ of Chloroform and $20 \mu \mathrm{l}$ of this solution was placed on a silicon wafer and then dried at room temperature overnight. The silicon wafer containing the desired MENs were glued on to a metallic sample puck and mounted on the AFM stage. All the images were performed in $256 \times 256$ scan resolution at a scan rate of $0.5 \mathrm{~Hz}$ at a $40-\mathrm{nm} \mathrm{Z}$-scale and a $60^{\circ}$-Phase angle. 74

Figure 25: Four transform infra-red studies. Fourier Transform Infra-Red (FTIR) results showing the spectrum change at different stages of the drug release. 75

Figure 26: Mass spectroscopy results showing (A) the significant peak of PTX at $876 \mathrm{~nm}$ in the MBPS buffer, (B) GMO-MENs in the MPBS buffer, (C) the significant peak of PTX at $876 \mathrm{~nm}$ when conjugated to the GMO-MENs in the MPBS buffer, (D) PTXGMO-MENs after the field treatment, (E) PTX after the release. 76

Figure 27: XRD pattern. XRD results of GMO-MENs at the three important phases of the release: (RED) before and (GREEN) after binding with PTX before the release and (BLUE) after the field-triggered release. Prior to performing the measurements, the nanoparticles were dispersed on a Silicon wafer and dried for 24 hours. 76

Figure 28: Drug uptake by cancer cells via different carriers. Comparison of four different forms of Flutax-2 drug intake by SKOV-3 cells $(n=3)$. 77

Figure 29: Confocal microscopy imaging of the drug uptake by SKOV-3 with different drug carriers. Confocal microscopy image of SKOV-3 cells showing the sub-localization of Flutax-2 (drug) when bound to different carrier systems: (a) free drug, (b) drug-HER- 
2, (c) drug-GMO-MNs in 30-Oe field. (d) drug-GMO-MENs with no field, (e) drugGMO-MENs in 30-Oe field. The scale bar is $47 \mu \mathrm{m}$.................................................. 78

Figure 30: Field dependence of the drug uptake by SKOV-3 and HOMEC cells. Flutax-2 drug uptake by HOMEC and SKOV-3 cells in varying magnetic fields (B). The drug uptake quantitatively presented in (A) was measured by a fluoremeter $(n=3)$. The scale bar on the images is $47 \mu \mathrm{m}$. 79

Figure 31: Confocal imaging of SKOV-3 cell viability after treatment by different drugcarrier combinations with and without field. Confocal images of: (A) SKOV-3 Cells treated with PTX drug with different carrier combinations, (left) no career with no field, (middle) HER2-GMO-MENs with no field, and (right) GMO-MENs in a 30-Oe field and (B) SKOV-3 Cell Controls that include the same three carrier combinations with no PTX drug present. 81

Figure 32: XTT cytotoxicity assay. The chart shows the results of XTT Assay performed on SKOV-3 cells at different concentrations of GMO-MENs $(n=3)$. 82

Figure 33: Dissipation-free physics. Surface temperature measured via Infra-red (IR) light for (A) cancerous (SKOV-3) and (B) healthy (HOMEC) ovarian cells before and 100-Oe field treatment. 83

Figure 34: Paclitaxel drug calibration curve obtained using spectrophotometer. A) Absorption maxima at $230 \mathrm{~nm}$ for different drug concentration (50 $\mu \mathrm{g} / \mathrm{ml}$ to $3.12 \mu \mathrm{g} / \mathrm{ml}$ ) in PBS buffer. B) Linear dependence of $230 \mathrm{~nm}$ absorption maxima with the drug concentration. 92

Figure 35: SEM image showing of the MENs. A) Uncoated MENs, B) GMO-MENs C) Tween20-MENs, and D) EDC-MENs. Scale bar 100nm. 96

Figure 36: FT-IR for surface functionalized MENs. MENs (A), GMO-MENs (B), Tween20-MENs (C), and EDC-MENs (D). 97

Figure 37: PTX drug loading. A) Time kinetic of PTX binding onto different MENs surface at 1:10 weight ratio (0.1 mg of PTX and $1 \mathrm{mg}$ of MENs). B) Binding isotherm of PTX onto different MENs surface at varying PTX drug concentration and for $3 \mathrm{hrs}$. incubation time. 99

Figure 38: Percentage PTX release at different magnetic field strengths and frequencies. A) Uncoated MENs, B) GMO-MENs, C) Tween20-MENs, and EDC-MENs.............. 100

Figure 39: XTT cytotoxicity assay. Viability of the SKOV-3 cells was determined using XTT assay after treating with various concentrations of MENs (uncoated MENs, GMOMENs, Tween-20-MENs and EDC-MENs) for $48 \mathrm{hrs.}$ 101

Figure 40: Illustration of MENs technology for deep brain stimulation 110 
Figure 41: Typical electrical activity in four regions (thalamic, sub-thalamic, globus pallidus-external, and globus-internal) of the brain in a healthy individual.

Figure 42: Discrete electrical activity in four regions (thalamic, sub-thalamic, globus pallidus-external, and globus-internal) of the brain in an individual suffering from Parkinson's. 113

Figure 43: Restored-electrical activity in four regions (thalamic, sub-thalamic, globus pallidus-external, and globus-internal) of a brain in an individual suffering from Parkinson's after treating with MENs non-invasively..... 114

Figure 44: Restored-electrical activity in four regions (thalamic, sub-thalamic, globus pallidus-external, and globus-internal) of a brain in an individual suffering from Parkinson's after treating with invasive DBS treatment. 114

Figure 45: Artistic view of the MENs interaction with neurons. The surface property of the MENs changes under the influence of surroundings microenvironment thereby changing their magnetic moment. 119

Figure 46: Relative magnetization curve showing the dependence on surface charge of the magneto-electric nanoparticles.

Figure 47: Relative magnetization curve for MNs. The chart shows no change in the magnetization curve for MNs having different surface charge (Q1, Q2, Q3, Q4, and Q5).

Figure 48: Showing the corresponding MRI/MNI signal for Relative magnetization (Mrel) change for different surface charge of the particle at $\mathrm{H}=50$ Oe. Mrel for magnetite (a-e). Mrel for MENs (f-j).

Figure 49: Relative Magnetization of the MEN for different particle sizes $(\mathrm{d} 1=20 \mathrm{~nm}, \mathrm{~d} 2=$ $50 \mathrm{~nm}, \mathrm{~d} 3=75 \mathrm{~nm}, \mathrm{~d} 4=100 \mathrm{~nm}$ ) keeping the charge $\mathrm{Q}$ as constant. (Note: that Magnetization decreases when size is decreases, as Q was kept constant. In reality surface charge depends on the size of the particle). 127

Figure 50: Relative Magnetization of the $\mathrm{MN}$ for different particle sizes $(\mathrm{d} 1=20 \mathrm{~nm}, \mathrm{~d} 2=$ $50 \mathrm{~nm}, \mathrm{~d} 3=75 \mathrm{~nm}, \mathrm{~d} 4=100 \mathrm{~nm})$.

Figure 51: Showing the corresponding MRI/MPI signal for change in Relative magnetization (Mrel) at $\mathrm{H}=50$ Oe for different size particles. Mrel for magnetite (a-d). Mrel for MENs (e-f).... 128

Figure 52: Relative Magnetization of Magnetoelctric nanoparticles at different concentration $(\mathrm{Cl}=0.05 \mathrm{~mol} / \mathrm{lit}, \mathrm{C} 2=0.1 \mathrm{~mol} / \mathrm{lit}, \mathrm{C} 3=0.5 \mathrm{~mol} / \mathrm{lit}, \mathrm{C} 4=2 \mathrm{~mol} / \mathrm{lit}) \ldots . .129$

Figure 53: Relative Magnetization of Magnetic Particle (Magnetite) at different concentration $(\mathrm{Cl}=0.05 \mathrm{~mol} / \mathrm{lit}, \mathrm{C} 2=0.1 \mathrm{~mol} / \mathrm{lit}, \mathrm{C} 3=0.5 \mathrm{~mol} / \mathrm{lit}, \mathrm{C} 4=2 \mathrm{~mol} / \mathrm{lit}) \ldots . .129$ 
Figure 54: Showing the corresponding MRI/MPI signal for change in Relative magnetization (Mrel) at $\mathrm{H}=50$ Oe for Concentrations. Mrel for magnetite (a-d). Mrel for MENs (e-f). (NOTE: (a,e at $=0.05 \mathrm{~mol} / \mathrm{lit}$ Concentration, $\mathrm{b}, \mathrm{f}$ at $=0.1 \mathrm{~mol} / \mathrm{lit}$ Concentration, $\mathrm{c}, \mathrm{g}$ at $=0.5 \mathrm{~mol} / \mathrm{lit}$ Concentration, $\mathrm{d}, \mathrm{h}$ at $=3 \mathrm{~mol} / \mathrm{lit}$ Concentration $) \ldots 130$

Figure 55: Segmented MRI data. White matter (orange), gray matter (blue), CSF (red), skull (gray), and scalp (brown). 130

Figure 56: Isotropic electrical conductivity (A) and the electric field (B) map of the MRI segmented data. 131

Figure 57: MRI image and electrical field image generated by using MENs ad a contrasting agents. Images show a clear additional signal (green) solely generated by the change in surface charge of the MENs due to the change in membrane properties of the neurons during the propagation of the action potential. 132 


\section{LIST OF ACRONYMS}

AC: Alternating current

AIDS: Acquired immunodeficiency syndrome

AZTTP: 3'-azido-3'-deoxythymidine-5'-triphosphate

AFM: Atomic force microscope

AuNPs: Gold nanoparticles

ARV: Anti-retroviral

BBB: Blood brain barrier

CSF: Cerebrospinal fluid

CNS: Central nervous system

CT: Computed tomography

CNT: carbon nanotubes

DC: Direct current

DNA: Deoxyribonucleic acid

DBS: Deep brain stimulation

DOI: Diffusion optical imaging

EDS: Energy-dispersive spectroscopy

EDC: N-(3-Dimethylaminopropyl)-N'-ethyl-carbodiimide hydrochloride

EGFR: Epidermal growth factor receptors

EEG: Electroencephalography

EOC: Epithelial ovarian cancer

FTIR: Fourier Transform Infra-Red

fMRI: Functional magnetic resonance image 
GMO: Glycerol monooleate

GM: Gray matter

HA: Human astrocytes

HAART: Highly active anti-retroviral therapy

HER-2: Human epidermal growth factor receptor 2

HOMEC: Human ovarian Epithelial cells

HSC: Human oral squamous cell carcinoma

HIV: Human immunodeficiency virus

ME: Magneto-electric

MENs: Magneto-electric nanoparticles

MNs: Magnetic nanoparticles

MEG: Magnetoencephalography

MRI: Magnetic resonance image

MNI: magnetic nanoparticle image

NHS: N-hydroxysuccinimide

NRTI: Nucleotide reverse transcriptase inhibitor

PBMCs: Peripheral blood mononuclear cells

PTX: Paclitaxel

PEG: Poly ethylene glycol

PET: Positron emission tomography

PLGA: Poly(lactic-co-glycolic acid)

RNA: Ribonucleic acid

RES: Reticuloendothelial system 
rTMS: Repetitive transcranial magnetic stimulation

SEM: Scanning electron Microscope

SKOV-3: Human ovarian carcinoma cell

SPIONPs: Superparamagnetic iron oxide nanoparticles

SPECT: Single photon emission computed tomography

TE: Tris-EDTA

TEM: Transmission electron microscopy

TMS: Transcranial magnetic stimulations

tDCS: Transcranial direct current stimulation

WM: White matter

XTT: 2,3-Bis(2-methoxy-4-nitro-5-sulfophenyl)-2H-tetrazolium-5-carboxanilide 


\section{LIST OF TABLES}

TABLE

PAGE

Table 1: Magneto-electric coefficients in two-phase systems. (Note: BaTiO3 and $\mathrm{Pb}(\mathrm{Zr}, \mathrm{Ti}) \mathrm{O} 3$ (PTZ) are piezoelectric materials, and TbxDy1-xFe2 (terfenol-D), ferrites, and manganite are magnetostrictive materials. $\mathrm{ME}$ coefficient, $\left.\mathrm{mV} \mathrm{cm}{ }^{-1} \mathrm{Oe}^{-1}\right)$. 16

Table 2: Mass spectroscopy instrumental parameters used for LC/MSD analyses (general

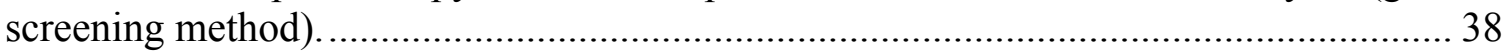

Table 3: Cell viability test (XTT) to illustrate non-toxicity of MENs at different concentrations.

Table 4: Drug release percentage at various combinations of field strength, frequency, and treatment duration

Table 5: The Malvern Zeta-sizer was used to measures size and Zeta-potential of the MENs, GMO-MENs, HER2-GMO-MENs, and PTX-GMO-MENs ( $\mathrm{n}=3$ ). Concentration of the nanoparticles used for the measurements was $500 \mathrm{ug} / \mathrm{ml}$ of D.I water. Note: The measurements represented are the average of three independent measurements. 71

Table 6: The field release dependence on the field, frequency, and the treatment duration. The table shows the percentage of the field-triggered released PTX at different combinations of the magnetic field strength $(12,44$, and 66 Oe) and frequency $(0,100$, and $1000 \mathrm{~Hz})$ and the treatment duration $(1,5,10,60$, and 120 minutes)....

Table 7: The table shows the percentage of cells viable after the 24-hour field-treatment period for different PTX-MEN combinations and field treatment conditions $(n=3)$. 81

Table 8: Change in zeta-potential and size measurements of the MENs surface before and after surface-functionalization. Zeta-potential values are the average and standard deviation of three independent trails $(n=3)$. 97 
Chapter 1: Introduction 


\subsection{Motivation}

The majority of the current nanoparticle based drug delivery systems are inefficient due to their dependence on the intrinsic properties of the cell/tissue physiological conditions to dispense cargo, thereby reducing the bioavailability of the therapeutic drugs. The primary motivation of the dissertation was to develop an energy-efficient drug delivery system that could release $\sim 100 \%$ of the therapeutic drug on-demand using the low field and low frequency remote magnetic field. The secondary motivation of the dissertation was to develop a non-invasive deep brain stimulation method to treat neurological disorders, such as Parkinson's, Alzheimer's, dementia, etc. Currently, the most widely used method is deep brain stimulation (DBS) that uses an extremely invasive direct contact electrode to stimulate the dysfunctional neurons in the deep brain region. Another goal of the dissertation was to develop a contrasting agent for magnetic resonance imaging and magnetic nanoparticle imaging to map neuronal activities of the brain (irrespective of its origin, i.e. superficial or deep tissue). Currently used techniques (electroencephalogram and magnetoencephalogram) can only record the neuronal activities in the superficial tissue (cortex) but not in the deep brain tissues.

\subsection{Specific aims}

Specific Aim 1: To develop an energy-efficient drug delivery vehicle that could release close to a $100 \%$ of the bound therapeutic payload on-demand using externally controlled remote magnetic fields.

Most of the current nanoparticle based drug delivery methods (both targeted and nontargeted) rely on the physiological conditions of the cell, such as temperature, redox, $\mathrm{pH}$, 
enzymatic reactions, etc. to release the therapeutic payloads. As a result, the drug releasing process is extremely inefficient due to the dependence on the minute changes in the physiological conditions in the microenvironment of the cell. Conversely, the MENsbased drug delivery method, which has been proposed by our research team, is independent of physiological conditions, as the drug-releasing process can be completely controlled using external magnetic fields. Similar to the conventional magnetic nanoparticle $(\mathrm{MN})$, the MEN have a non-zero magnetic moment and therefore can be controllably transported through a body via application of a remote magnetic field gradient. However, unlike the MN, the MEN display an entirely new property, which is the non-zero magneto-electric (ME) effect. The origin of this effect is the intrinsic coupling between electric and magnetic fields within the MEN, even at body temperature. Because of its quantum-mechanical nature, the coupling is extremely energy efficient and can be made dissipation free. Moreover, these payloads are steered to the site of delivery using external magnetic fields, thereby increasing the bioavailability of the drugs. This technology was evaluated on the two most researched areas, i.e. neurological-HIV and ovarian cancer, to deliver therapeutic drugs on-demand.

Specific Aim 2: To surface-functionalize the MENs for carrying a wide range of therapeutic drugs to treat multiple diseases and to maximize the payload carrying capacity.

Therapeutic drugs are attached to the nano-vehicles via covalent, non-covalent (electrostatic, Van der Waal, hydrogen), or ionic bonds. Modifying the surface properties (i.e. by controlling the surface charge and polarity) of the nano-vehicle could assist in 
attaching a wide range of therapeutic drugs on the surface. This is a key requirement for applying the technology to treat numerous diseases. Surface properties of the nanovehicles are altered using several functionalizing agents. Tailoring the surface properties of nano-vehicles not only enables them to carry wide varieties of payloads, but could also improve the payload carrying capacity and drug-binding efficacy.

Specific Aim 3: To develop a non-invasive neuronal stimulation method to restore the neuronal activity of the dysfunctional neurons using a low field and low frequency external magnetic field.

The signaling in a biological neuronal network is based on a highly collective system of electric charges, neurotransmitters and action potentials. The ability to incite the neuronal charge excitations from outside the body non-invasively with the purpose to artificially stimulate the neuronal network remains an important roadblock to enabling leapfrog advances in the important area of neuroscience and related applications in medicine and neural engineering. One potential solution to overcome this important roadblock for enabling the non-invasive control of the neuronal network is to exploit the magnetoelectric property of the MENs.

Specific Aim 4: To develop a new contrasting agent for imaging modalities (magnetic resonance imaging, MRI and magnetic nanoparticle imaging, MNI) that could not only act as a contrast enhancement agent, but could also provide the molecular information of the cell/tissue microenvironment. 
The signal in the imaging modalities (MRI and MNI) is mainly dependent on the magnetic saturation and the density of the contrasting agent. On the contrary, in MENs (as contrasting agent), due to the intrinsic magnetic-electric coupling at the human body temperature, the MRI signal also depends on electric fields that are involved in the molecular bonding between the MENs and the surrounding environment. Additionally, as the molecular bonds also control the fate of the MENs payloads, it could be possible to visualize the dynamic drug release process in vivo. Moreover, these particles could also give us information about electrical activity in the brain, as neuronal firing changes the surface charge of the MEN due to the generation of action potential.

\subsection{Nanomaterials for payload delivery, targeted therapy and diagnostics}

Researchers have long been struggling to find a better way to dispense the therapeutic payloads to the diseased tissues with high efficiency, precision, solubility, and stability while maximizing the biodistribution/bioavailability of the therapeutic payloads. Paul Ehrlich was the first person to propose the use of a delivery vehicle that could selectively target the diseased-tissue/organism and deliver a toxin for that specific disease/organism. Thus, the term "magic bullet" was coined to deliver the therapeutic payloads to a specific location or to destroy an organism exclusively. Recently, several nanomaterials with various compositions and biological properties have been designed to carry therapeutic drug payloads to the target sites (2-6). These nanomaterials have unique advantages over the conventional delivery systems, for example: (i) delivering the payloads to specific locations in the body; (ii) minimizing the drug dose levels, as the drugs are delivered right at the target site; and (iii) minimizing the drug levels at non-target sites, thereby 
reducing the toxic side effects. Besides the drug-delivery applications, nanomaterials are also widely used for several other applications, such as cell imaging, diagnostics, protein and nucleic acid (DNA/RNA) detection. Most widely used nanomaterials for drugdelivery and diagnostic applications include: Au nanoparticles, iron oxide nanoparticles, magneto-electric nanoparticles, Poly(lactic-co-glycolic acid) nanoparticles, carbon nanotubes, and mesoporous silica nanoparticles. Drug delivery and diagnostic applications using some of these nanomaterials are elaborated in detail in the following section.

\subsubsection{Gold nanoparticles}

First, gold nanoparticles (AuNPs) are used widely to delivery a wide range of payloads (including proteins, nucleic acids (DNA \& RNA), small and large drug molecules) to the target sites (7-13). The release of the payloads using AuNPs can be controlled using a micro-environmental physiological stimulus of the cell ( $\mathrm{pH}$ or glutathione-mediated) or an external stimulus (light or temperature) $(12,14-17)$, as shown in Figure 1A. Second, AuNPs are used in enhancing the dose amounts of low-energy x-rays and gamma rays $(<100 \mathrm{KeV})$, which appears to be a highly feasible and alternative approach to fulfill some technical and clinical limitations for eradicating tumors requiring higher dose levels (18). Third, AuNPs are used in photothermal therapy of cancer $(19,20)$. Here, AuNPs are accumulated at the tumor tissues and irradiated with an external laser source (650-900 $\mathrm{nm})$. Due to the strong electric fields at the surface of the noble metal, the absorption and scattering of the electromagnetic radiation is greatly enhanced, thus producing the heat to eradicate the surrounding tumor cells. The specificity of the photothermal therapy, i.e. to 
target only tumor tissues, is achieved by tagging the AuNPs with HER-2 antibodies, which now specifically bind only to the tumor cells $(21,22)$. Fourth, AuNPs are also used in cancer cell imaging (23). Epidermal growth factor receptors (EGFR) conjugated AuNPs are employed to bind specifically to human oral squamous cell carcinoma (HSC and HOC). Such cells, when irradiated with near-infrared light, produce strong absorption and scattering. The emitted radiation is captured using light scattering and surface plasmon resonant absorption spectroscope to produce an image specific to carcinomas (shown in Figure 1B).

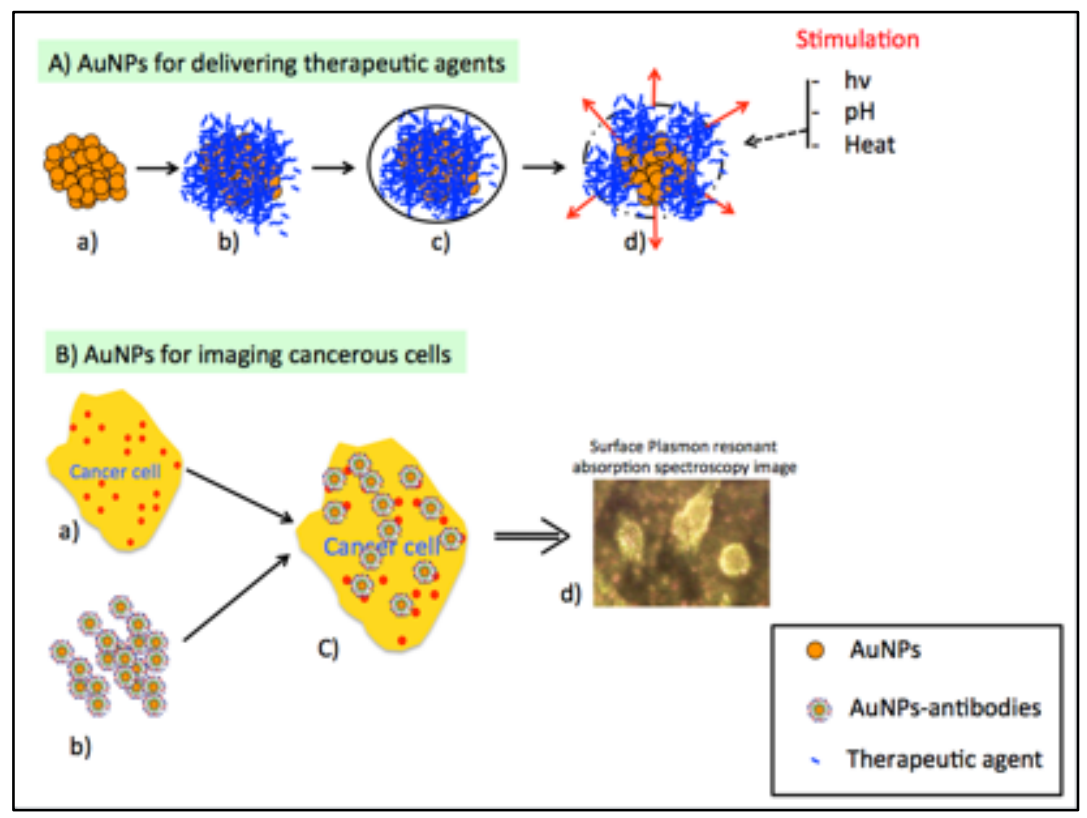

Figure 1: A) AuNPs for delivering the therapeutic agents. a) bare-AuNPs, b) AuNPs loaded with therapeutic agents (proteins/DNA/RNA/drugs), c) encapsulation of AuNPs loaded with therapeutic agents, and d) release of therapeutic agents by the physiological (pH and temperature) and external stimulations (light). Figure 1B, a) cancer cell surface with biomarkers, b) AuNPs, tagged with anti-biomarkers, c) accumulations of biomarker tagged-AuNPs on cancer cell surface, and d) a surface plasmon resonant absorption spectroscope image showing only cancer cells through AuNPs accumulation. 
All of the above described delivery and therapeutic applications of AuNPs are possible because of the unique chemical and physical properties of the gold metal. Moreover, the ease of fabrication techniques to synthesize uniform AuNPs of different sizes (1 to 150 nm) and shapes (cubes, spheres and rods) lead to their use in multiple clinical applications (24-27). Further, AuNPs are also inert and non-toxic in nature, which makes it possible to employ them, as drug delivery vehicles in human and animal subjects with no toxic side effects (28-32).

- Advantages of AuNPs

a. Biocompatibility: AuNPs show less or no toxicities in the in-vitro and invivo applications.

b. Ease of fabrication: chemical synthesis of AuNPs of different sizes and shapes is extremely simple.

c. Ease of tailoring surface properties: ability to functionalize the nanoparticles to carry a wide range of therapeutic payloads is mandatory in treating a wide range of diseases.

d. Eradication of radiation-resistant tumors: enhancement of low energy xrays and gamma rays to eradicate radiation resistant carcinomas.

e. Diagnostics: tumor detection based on the cell-specific biomarkers.

- Disadvantages of AuNPs

a. Inefficient release: The primary disadvantage of using AuNPs for payload delivery is their dependence on micro-environmental physiological 
conditions of the cell as a stimulus, resulting in the drug release process being extremely inefficient.

b. Low penetration depths of visible light: the tissue penetration depth is a huge limitation in treating deep-tissue carcinomas. (Note: The visible light tissue penetration depth is only $2-3 \mathrm{~cm}$ ).

c. Antibody tagging: targeted delivery is completely driven by the cellspecific antigen and antibody reactions, thereby limiting its application only to the biomarker specific targets.

d. Specificity: use of AuNPs, as contrasting agents also demands surface functionalization of the nanoparticles with cell-specific antibodies. Thus, only the carcinomas carrying the counter antibodies (or antigen) can be detected.

Note: AuNPs for biomedical applications and their fundamental limitations are summarized in Figure 2. 


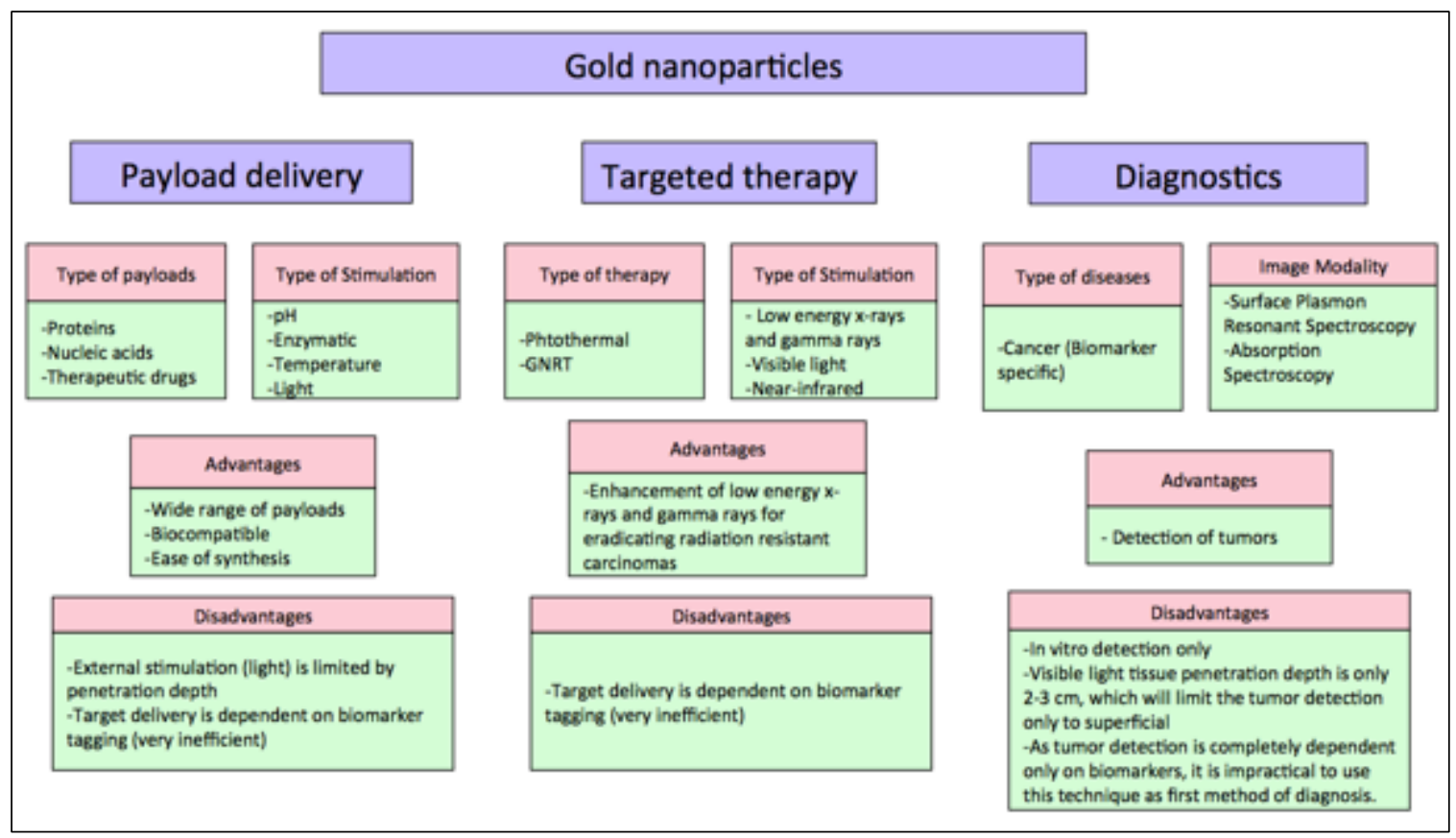

Figure 2: Advantages and disadvantages of AuNPs in various biomedical applications.

\subsubsection{Iron oxide nanoparticles}

The use of iron oxide $\left(\gamma-\mathrm{Fe}_{2} \mathrm{O}_{3}\right.$ or $\left.\mathrm{Fe}_{3} \mathrm{O}_{4}\right)$ particles for in vitro medical diagnostics applications has been practiced for more than 50 years (33). However, over the last decade, the use of nanosized iron oxide particles, especially $\mathrm{Fe}_{3} \mathrm{O}_{4}$ (magnetite) has become extremely popular due to their ability to function at the molecular level, having distinctive physical properties, and for showing biocompatibility (34-36). The unique physical properties, i.e. quantum-size and large surface area of the magnetite dramatically change its magnetic properties, to exhibit quantum tunneling and superparamagnetism. These unique properties offer high potential for numerous biomedical applications, such as cell labeling cell isolation, payload delivery, tissue repair, MR contrast enhancement, and hyperthermia. Surface functionalized magnetite nanoparticles could be dispersed into appropriate solvents to form homogeneous ferrofluids $(37,38)$. To perform therapeutic 
procedures, these ferrofluids can be steered to any part of the subject's body (human/animal) using an external magnetic field gradient system (39-41). Intravenous administration of ferrofluids is relatively non-toxic and such preparations are used in current clinical applications (42).

The main advantages of using magnetic nanoparticles (MNs) over the non-magnetic particles includes: (i) MNs can be visualized using MRI and MNI modalities, thus ensuring that the payloads are delivered to the right target sites; (ii) MNs can be steered/positioned at any location in the body with a micrometer scale precision using an external magnetic field gradient system, thus eliminating the dependence on cell-specific antigen-antibody reactions; and (iii) The temperature of the MNs can be increased using the high frequency magnetic fields. The radiated heat can assist in releasing the therapeutic drug and/or in ablation of the surrounding tissues (for example cancer tissue). Various biomedical applications of the MNs are discussed in detail in the following section.

\section{- Cellular labeling and cell isolation}

Antibody tagged MNs are up-taken by the desired cells through cell-specific antigenantibody reaction or receptor mediated endocytosis or phagocytosis (43-45). The uptaken cells are imaged using MR/MN image modalities for their specific location in the body. Desired cells can be isolated from the biological fluids by incubating with antibody-coated magnetic beads. The desired cells are attached to the magnetic beads 
through cell-specific antigen-antibody reaction. Washing the cell-labeled magnetic beads several times on magnetic racks isolates the desired cells.

\section{- $\quad$ Tissue repair}

Synthetic-polymer/protein-coated MNs are used in the tissue repair procedure (46). Briefly in this technique, two opposing tissues coated with nanoparticles are heated to 50 ${ }^{\circ} \mathrm{C}$ using a soldering gun or a laser source to seam them together (47). This technique greatly minimizes the tissue damage, as it requires low temperatures and low energy laser sources. The technique has a great potential to repair/transplant the desired tissues using the stem cell payloads (48). Stem cells are specialized cells having a unique ability to self-renew and differentiate into any other cell type. Stem cell-loaded MNs could be navigated to the specific locations (damaged tissue) to perform the tissue development/repair process. This type of technology has tremendous potential to treat diseases, such as Alzheimer's, Parkinson's, and heart disease non-invasively.

\section{- Payload delivery}

Like AuNPs, MNs can also carry a wide range of therapeutic payloads (drugs, proteins, nucleic acids) to the target sites (49-51). To enable this, size, charge, and surface chemistry of the MNs need to be tailored. For example, to conjugate the protein payloads with the MNs, the surface of the nanoparticle is modified with poly ethylene glycol (PEG) (52). These PEG modified MNs will also further benefit from being unrecognized by the reticuloendothelial clearance system thus facilitating the tissue specific uptakes. Moreover, surface modification using biodegradable materials (such as poly(lactic-co- 
glycolic acid)) also reduces the toxicity and immunological responses to inorganic material (such as MNs) $(53,54)$.

\section{- Magnetic resonance imaging}

Superparamagnetic iron oxide nanoparticles (SPIONPs) are used, as a contrast enhancement agent in MR imaging modality to better differentiate the pathological tissue from the healthy tissue. SPIONPs are also capable of achieving near-microscopic resolution in in-vivo MR imaging applications (55). MNs tagged with stem cells can be visualized and tracked in the body using MR imaging (56). Further, stem cell tagged MNs will not affect the growth, differentiation, viability, and proliferation of the attached cell. SPIONPs are also used in visualizing the transgene expression and in detecting cell apoptosis through MR imaging $(57,58)$.

\section{- Hyperthermia}

The use of hyperthermia (heat) to eradicate the cancer cells is, as old as medicine itself. Hippocrates first proposed the use of hot iron to burn the surface of carcinomas to eradicate the diseased tissue. Currently, more advanced methods, such as perfusion heating, ferrofluid hyperthermia, irreversible electroporation, and high-frequency radiation are being used to heat, denature, and destroy the cancer cells (59-61). MNs can also be used to generate substantial amount of heat to destroy tumors using the high frequency magnetic fields $(62,63)$. The amount of heat generated using this phenomenon (due to the hysteresis loss) depends on the material properties of the particles and also on 
the strength of the applied magnetic field and frequency. The applications of MNs and their advantages and disadvantages are shown in Figure 3.

\section{Magnetic nanoparticles}

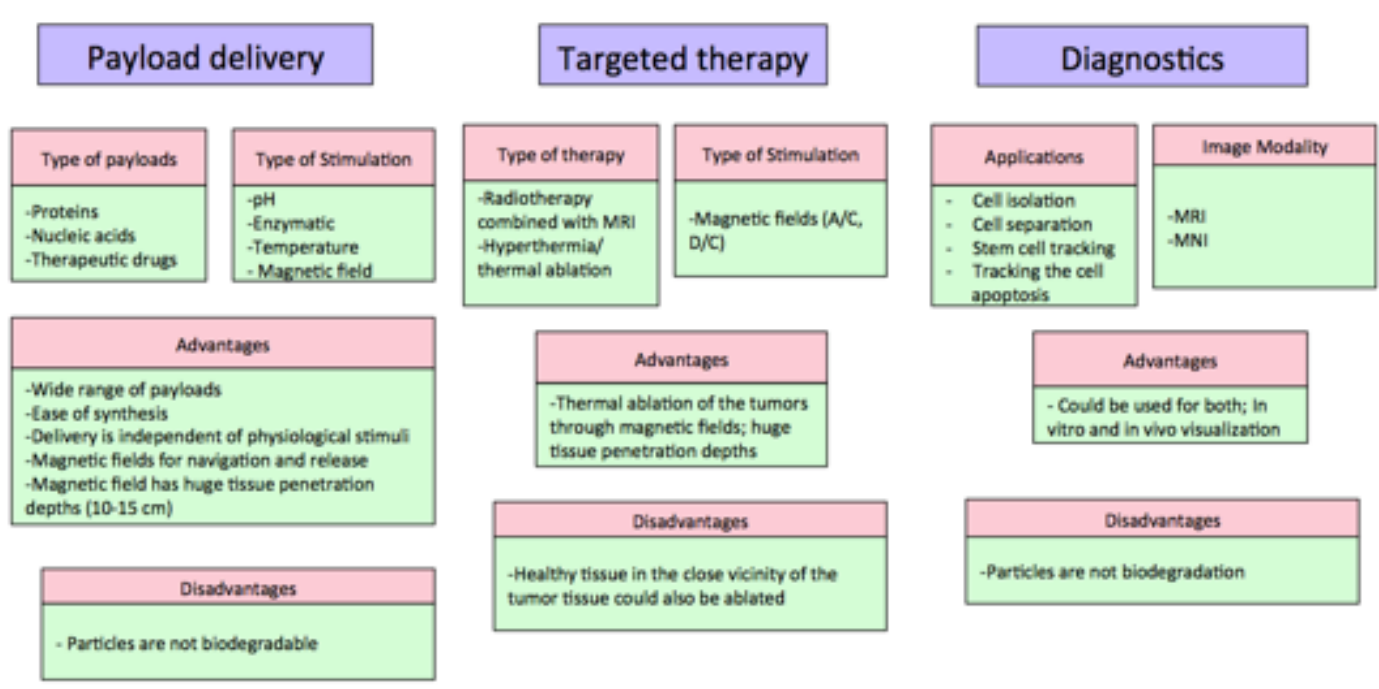

Figure 3: shows advantages and disadvantages of using magnetic nanoparticles for various applications.

Current state-of the-art MNs based technology for delivering anti-cancerous drugs or for ablating tumor tissues (through hyperthermia) can only be applicable to a well-defined tumor. However, treating small-tumors and metastatic neoplasms with this technology still remains a challenge. Finally, MNs based technology can play a major role in treating tumors in the regions, where conventional surgery methods cannot be applied. 


\subsubsection{Magneto-electric nanoparticles}

Any material that exhibits a strong quantum coupling of magnetic and electric properties is generally called as magneto-electric material. These materials come under the multiferroic category, as they simultaneously demonstrate both ferroelectric and ferromagnetic properties (64). Figure 4 shows the relationship between the magnetoelectric and multiferroic materials. Borocite $\left(\mathrm{Ni}_{3} \mathrm{~B}_{7} \mathrm{O}_{13} \mathrm{I}\right)$ was the first material reported to exhibit magneto-electric switching below $60 \mathrm{~K}$ (65). Magnetic to electric energy conversion exploits the advantages of properties from two different phases of the material, i.e. from the ferromagnetic phase, piezomagnetic or magnetostrictive property and from the ferroelectric phase, piezoelectric or electrostrictive property. Thus, when an external magnetic field $(\Delta \mathrm{H})$ is applied, magnetostrictive stress formed in the magnetic phase is transferred to the ferroelectric phase changing the polarization $(\Delta \mathrm{P})$ and the associated electric field $(\Delta \mathrm{E})$ due to the piezoelectric effect. Performance of multiferroic materials is determined by this transfer efficiency, which is called as magneto-electric coefficient $(\alpha=\Delta \mathrm{P} / \Delta \mathrm{H})$ or as magneto-electric voltage coefficient $\left(\alpha_{\mathrm{E}}=\Delta \mathrm{E} / \Delta \mathrm{H}\right)$. Magneto-electric coefficients of various multiferroic materials are shown in Table 1. Electric flux density (D) in multiferroic materials (Equation(1) is determined by the magneto-electric coefficient tensor $\left(\alpha_{\mathrm{ij}}\right)$, permittivity tensor $\left(\varepsilon_{\mathrm{ij}}\right)$, and the piezoelectric coefficients $\left(e_{\mathrm{ij}}\right)$ in an applied vector magnetic, electric field, and external stress S (64).

$$
D_{i}=\sum_{j-1}^{j-3} \varepsilon_{i j} E_{j}+\sum_{j-1}^{j-6} e_{i j} S_{j}+\sum_{j-1}^{j-3} \alpha_{i j} H_{j}
$$




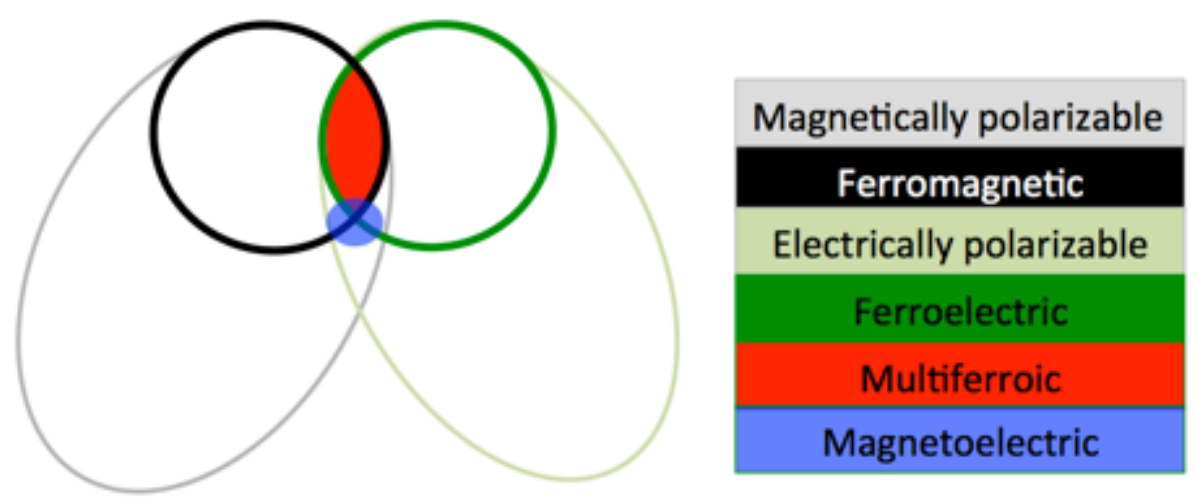

Figure 4: The relationship between the magneto-electric and multiferroic materials.

Table 1: Magneto-electric coefficients in two-phase systems. (Note: BaTiO3 and $\mathrm{Pb}(\mathrm{Zr}, \mathrm{Ti}) \mathrm{O3}$ (PTZ) are piezoelectric materials, and TbxDy1-xFe2 (terfenol-D), ferrites, and manganite are magnetostrictive materials. ME coefficient, $\left.\mathrm{mV} \mathrm{cm}^{-1} \mathrm{Oe}^{-1}\right)$.

\begin{tabular}{|c|c|c|c|}
\hline Morphology & Materials & $\begin{array}{c}\text { ME } \\
\text { coefficient }\end{array}$ & Ref. \\
\hline Composite & $\mathrm{BaTiO}_{3}$ and $\mathrm{CoFe}_{2} \mathrm{O}_{4}$ & 50 & $(66)$ \\
\hline Composite & Terfenol-D and PZT in polymer matrix & 42 & $(67)$ \\
\hline $\begin{array}{l}\text { Laminated- } \\
\text { composites }\end{array}$ & Terfenol-D and PZT/PZT (in polymer matrix) & 3000 & $(68)$ \\
\hline Laminate & $\mathrm{Terfenol-D/PZT}_{0.7} \mathrm{Sr}_{0.3} \mathrm{MnO}_{3} / \mathrm{PZT}$ & 4800 & $(69)$ \\
\hline Laminate & $\mathrm{NiFe}_{2} \mathrm{O}_{4} / \mathrm{PZT}$ & 60 & $(70)$ \\
\hline Laminates & & 1,400 & $(70)$ \\
\hline
\end{tabular}

Multiferroic materials can be used in two forms, i.e. either in the laminate form or in the particulate form. However, using multiferroic materials in the particulate form provides excellent magneto-electric properties due to the below reasons:

a. Shape effects: spherical shape of the particles provides better transfer of magnetostrictive stress from magnetic phase to the ferroelectric phase. 
b. Zero-substrate clamping effect: laminates/films needs to be clamped, which restricts the magnetostrictive strains.

c. Binding efficiency: mechanical contact in the interface of particulate composites is much higher as compared to the laminate/films composites. (Note: laminate/films composites need to be either glued or sintered which often results in poor binding efficacies).

So far, multiferroic materials are extensively used in microwave filters, signal amplification devices, data storage devices, transducers, optical-diodes, power generation systems, high frequency switches, miniature-antennas and spintronics (71-77), but for the first time in this dissertation, we presented the biomedical applications of the multiferroic materials (Note: details of these applications are provided in the next chapters). Unique advantages of multiferroic materials for biomedical applications are discussed in detail in the following sections.

\subsection{Artificial brain stimulation for treating neurological disorders}

The signaling in a biological neural network is based on a highly collective system of electric charges, neurotransmitters and action potentials. The ability to incite the neuronal charge excitations from outside with the purpose of artificially stimulating the neural network remotely (non-invasively) remains an important roadblock to enable significant advances in the important areas of neuroscience and related applications in medicine and neural engineering. The progress of the existing invasive and non-invasive technologies, 
such as deep brain stimulation (DBS), transcranial magnetic stimulations (TMS), repetitive transcranial magnetic stimulation (rTMS), and transcranial direct current stimulation (tDCS) are discussed in detail in the following sections along with their advantages and disadvantages.

\subsubsection{Invasive stimulation}

Invasive brain stimulation methods are currently being practiced for curing multiple neurological disorders, such as Parkinson's disease, neuropathic pains, major depression, Alzheimer's disease, Tourette syndrome, Epilepsy, Dystonia, obsessive-compulsive disorders, tremors, and self-injury syndromes. These invasive brain stimulation methods include: deep brain stimulation (DBS), thalamotomy, bilateral lesions, and pallidotomy. Most of these techniques (except the DBS) are nearly discontinued due to their irreversible complications and curtail therapeutic effects $(78,79)$. Currently, the only practiced neurosurgical method for brain stimulation is DBS. In this technique, programmable neuropacemakers (pulse generators) are connected to the implants (electrodes), which are chronically inserted into the desired regions of the brain to artificially restore its neural activity by periodic electric stimulation. Implants are held in a desired location for weeks to years to stimulate the region of brain depending on the type of disorder and response to the treatment $(80)$. The location of the implant also plays a major role in curing/reducing the condition of the disease (81-83). For example, in case of Parkinson's, stimulating the ventral intermediate nucleus of the thalamus (VIM) may reduce only limbic tremor but has no effect on other manifestations of the disease; 
whereas stimulating the globus palidus (GPi) may reduce most of the major manifestations of the Parkinson's including the painful cramps, sensory symptoms, dopainduced dyskinesia and involuntary movements (84). In case of nociceptive pain, stimulating the periaqueductal gray or periventricular gray may help in minimizing the pain and for differentiation pain, stimulating the sensory thalamus may help (84).

- Advantages of DBS

a. It is a highly reversible process; therefore there is no damage to the neuronal tissue.

b. Programmable implants can control the dosage of stimulation depending on the response to the treatment.

c. Stimulating specific regions of the brain can treat multiple neurological disorders.

- Disadvantages of DBS

a. Extremely invasive technique.

b. A mechanical and functional failure demands replacement of implants through neurosurgeries (usually once in 2-3 years).

c. Implantation of neurostimulators invasively is often followed by high risk of infection. Extreme measures must be taken to protect the patient from developing neural infections.

d. There is a small chance of permanent neurological complications that leads to memory loss, change in thinking, personality disorders, loss of vision, and paralysis. 
e. Cost of the surgery and maintenance of functional integrity of the implants could be very expensive.

\subsection{Non-invasive brain stimulation}

Over the last decade the use of non-invasive brain stimulation methods for treating multiple neurological (Parkinson's, tics, epilepsy, tinnitus, stuttering, or dystonia) and psychiatric (depression, drug craving, obsession, hallucination, catatonia, bipolar disorders, mania, or trauma) disorders has rapidly increased. Even though these reported (in literature) applications are poorly supported by the clinical data, the demand for the non-invasive brain stimulation procedures is still enormous, benefiting the millions of patients suffering from these dreadful disorders. Despite such a rapid growth and vast applications of this procedure, most of the current techniques are either less effective or require high intensity magnetic fields to stimulate the brain tissue. Hence, it is necessary to develop a better technology to increase the efficiency and effectiveness of the treatment. TMS, rTMS, and tDCS are the three most commonly used non-invasive brain stimulation techniques to treat wide variety of neurological disorders. In TMS, local magnetic pulses are generated using an insulated TMS coil (butterfly-shaped) placed over the scalp by passing high amounts of electrical currents $(>5 \mathrm{KA})$ at the frequency of $1000 \mathrm{~Hz}$ or greater. The local magnetic pulses generated by the TMS coil penetrate through the skull and induce small eddy currents in the local conductive regions of the brain tissue to depolarize the neurons. In rTMS, these currents are applied repetitively to

control the cortical excitability (increase/decrease) for longer periods (85). In tDCS, low 
amplitude direct currents $(\sim 2 \mathrm{~mA})$ are generated constantly using a current generator that is connected to a pair of saline-soaked sponge electrodes $\left(20-35 \mathrm{~cm}^{2}\right)$. These electrodes are placed on the subject's head as shown in Figure 5 to stimulate the brain tissue (86). The direction of the flow of current through the sponge electrodes defines the type of the tDCS, i.e. "cathodal" or "anodal". The polarity of the tDCS plays a major role in the modulation of cortical excitability. For example, cathodal tDCS will reduce the cortical excitability, whereas anodal tDCS will increase it.

\section{TMS}

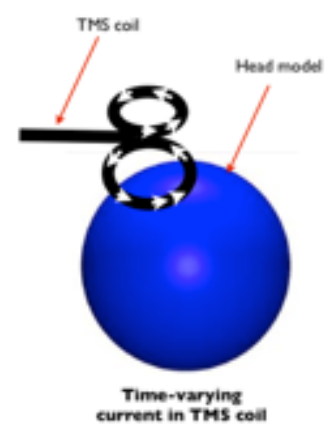

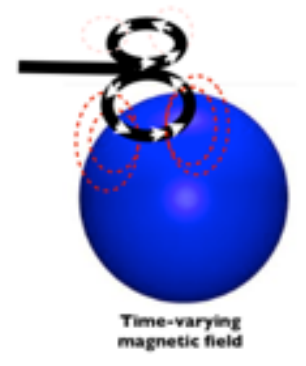

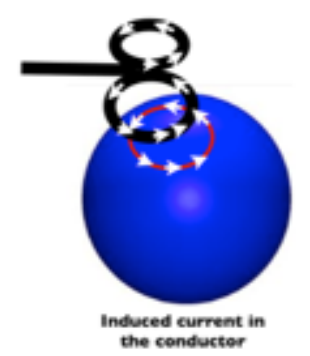

tDCS

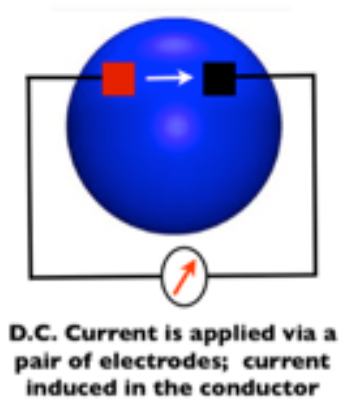

Figure 5: TMS and tDCS principles of non-invasive brain stimulation.

Recent pharmacology studies show that tDCS does not stimulate the neuronal firing; it rather modulates the stimulated neurons (87). This property of tDCS separates it from other stimulation techniques, such as TMS/rTMS. Note: during the stimulation process, the subjects might feel an itching or tangling sensation on the scalp beneath the sponge electrodes, which could be extremely painful (87). The advantages and disadvantages of the TMS, rTMS, and tDCS technologies are explained in the following section. 
- Advantages of TMS, rTMS, and tDCS techniques

a. They are cheap, safe, painless, and non-invasive.

b. No chance to develop post-surgical infections, as treatment is non-invasive

c. Zero recovery time. No restriction on patient's activity before/after the treatment procedure.

d. Highly reversible. No cognitive or systemic after effects (memory loss, personality disorders, paralysis etc.,) due to non-invasive treatment.

- Disadvantages of TMS, rTMS, and tDCS techniques

a. High magnetic field used in TMS/rTMS leads to undesirable side effects.

b. The penetration depths and locality of focusing fields often limit the effectiveness of the treatment.

c. Loud noises produced in TMS/rTMS often cause discomfort throughout the treatment procedures. Moreover, patients undergoing tDCS also feel a constant discomfort due to itching/tangling sensation beneath the sponge electrodes.

d. Patients need to undergo the treatment procedure more often to make the treatment effective.

In the next few chapters, we will introduce a new concept of using magneto-electric particles (MENs) for non-invasive brain stimulation to address several disadvantages of current non-invasive and invasive techniques to enable better neuronal stimulation treatment procedures for eradicating neurological disorders. 


\subsection{Brain electrical activity mapping}

Brain imaging is either a direct or an indirect method of imaging the structural, functional or the pharmaceutical information of the brain. Currently, brain imaging is performed by several techniques, such as Magnetic resonance imaging (MRI), Positron emission tomography (PET), Single photon emission computed tomography (SPECT), Computed tomography (CT), Diffusion optical imaging (DOI), Functional-MRI (fMRI), Magnetoencephalography (MEG), and Electroencephalography (EEG). Of all these techniques, only MEG and EEG can provide the substantial information about the brain's electric fields. Electric fields in the brain (of the order femtovolts) are generated by the net flow of ions through the nerve cell during the synaptic transmission (shown in Figure 6). These generated electric fields can be captured using either a MEG or an EEG technique to track the neuronal firing $(88,89)$. Neurons are fired by all kinds of activities performed by one's brain, whether it is a heartbeat, locomotion or a basic conscious thought. Tracking these brain dynamics through the electrical signal and correlating with the associated activities is the ultimate dream of all the neurologists. Understanding this neuronal activity plays a pivotal role in diagnostics and treatment of multiple neurological disorders non-invasively $(90,91)$. In the following section, basic function of the MEG/EEG along with their advantages, and disadvantages are discussed in detail.

\subsubsection{Source of EEG/MEG signal}

As previously stated, electric fields in the brain are generated by the net flow of ions through the neurons during the synaptic transmission. The electric field by a single 
neuron cannot be detected at the scalp level due to the infinitesimal amplitude of the signal, but can be detected if synaptic transmission takes in alpha rhythm $(8-13 \mathrm{~Hz})$ by thousands of neurons $(\sim 50,0000)$. When the wave of these cumulative electric fields reaches the scalp of the subject, the EEG electrodes mounted on the scalp can harvest, as shown in Figure 6. In MEG, signal detection on the scalp level is from the orthogonal magnetic fields produced by the flow of ions through the bundles of axons (called volume conduction), which are oriented tangentially to the scalp surface as shown in Figure 6 . Spatial orientation of these axons plays a crucial role in determining the strength of the EEG/MEG signal at the scalp surface. For example, if the axons are oriented oppositely, the net magnitude of current generated due to the flow of ions will be zero and further, will mitigate the process of volume conduction. Therefore, the signal in the EEG/MEG is thought to be produced only by the pyramidal neurons in the cortex, as they are well oriented and fired together (92). The signals from the deep tissue activities will fall off (square of distance) before reaching the surface and would be impossible to detect at the scalp level. Note: recording the signals at the scalp level over a period of time gives the EEG/MEG signature of a particular activity in the cortex or sub-cortex but not from the deep brain tissue (93). 


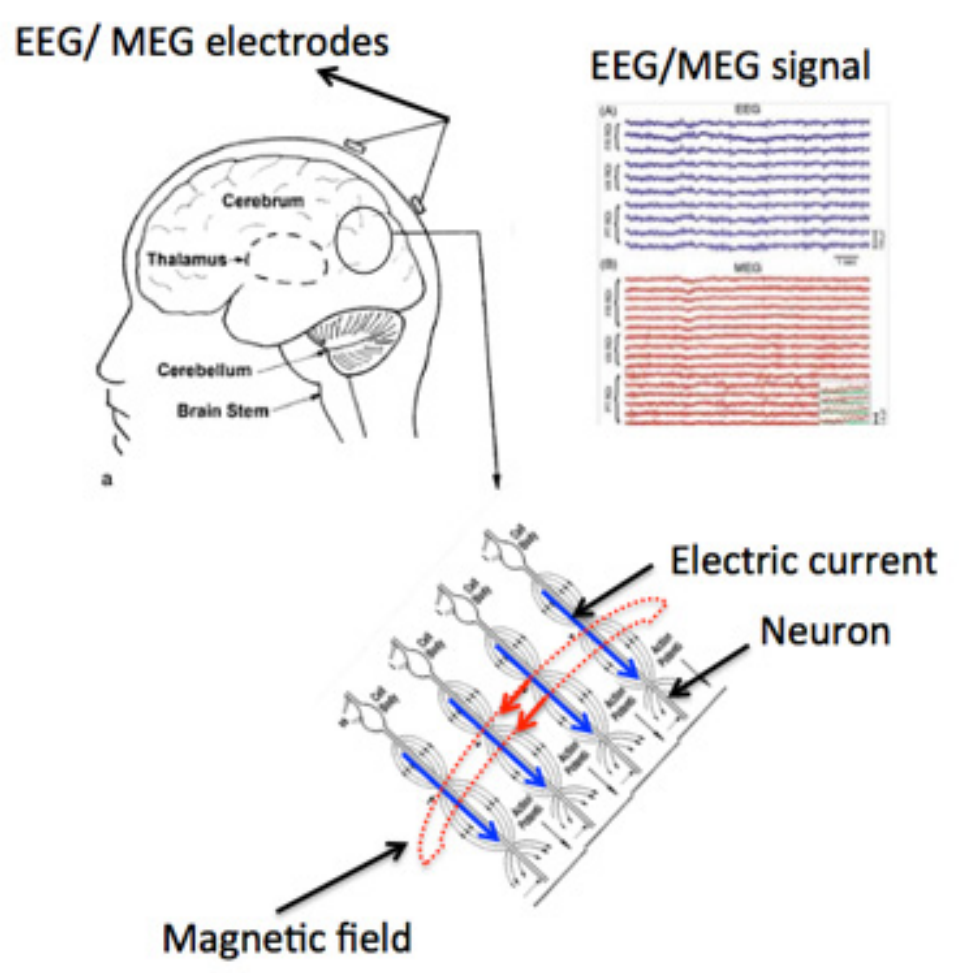

Figure 6: Signal in EEG/MEG. The synchronous firing of the neurons with the electrical currents and the associated magnetic fields are illustrated in the image.

- Advantages of brain mapping using EEG/MEG

1. Cheap, non-invasive, extremely sensitive to the cortical signals, and has unlimited virtual time resolution.

2. Both the methods can provide information regarding the location and the strength of the electrical activity in the brain (cortical).

3. Can be used, as a diagnostic tool in identifying the malfunctioning neurons in diseased subjects (neurological). This information can be used later in stimulating associated neurons by non-invasive brain stimulation methods in the treatment process. 
- Disadvantages of brain mapping using EEG/MEG

a. The primary disadvantage of both the techniques is requirement of neuronal firing to be alpha rhythmic (for volume conduction) and also demands the orientation of the neurons to be pyramidal.

b. Activities associated with the deep brain regions cannot be detected.

c. Very low spatial resolution.

d. Low signal-to-noise ratio.

e. Sophisticated data analysis.

\subsection{Unique advantages of magneto-electric nanoparticles over the conventional nanoparticles for biomedical applications}

There are many different types of nanoparticle systems used in medicine for drug delivery purposes alone. To mention a few, they rely on using thermally-responsive polymers, optically (UV, Visible-Wavelength, and IR) or acoustically activated materials, liposomes, electrochemical processes, and magnetic forces (94). In this case, the speed of delivery is limited by the external field sources and not just by the blood circulation. For instance, as it is related to the release across the blood-brain barrier (BBB), the magnetic delivery provides a unique way to transfer drugs sufficiently fast to avoid their engulfing by the reticuloendothelial system (RES) (95). However, all the existing delivery technologies suffer from the lack of certainty of drug release from the carrier if and when the nano-carrier reaches the target. Probably, the main stumbling block is the inability of the current nanoparticle systems to remotely control the intrinsic phenomena that define 
the interaction of the nanoparticles with the surrounding cells and tissues. The conventional approaches are extrinsic in their nature and often fail to adequately regulate cellular phenomena, such as exocytosis of drug with intracellular vesicle, control of ionic channels, using externally controlled signals (96). In other words, the fundamental physics that underlies the nanoparticle system characteristics in the perspective of their intrinsic interaction with the surrounding cells and tissues in the aforementioned applications is barely exploited. Revealing and controlling the interaction of nanoparticles at the nanoscale, whether it is electric field-, magnetic spin-, photon-, or phonon-triggered, is vital for enabling perfect diagnostics and/or recovery/regeneration of all the medical functions.

Our discovery of the unprecedented capabilities of MENs paves a way to fill this gap through nanotechnology approaches. In this dissertation, we have shown that the intrinsic quantum-mechanically defines coupling between electric dipoles and magnetic spins within each nanoparticle, MENs could be used, as an energy-efficient and a dissipationfree field-controlled nano-vehicles for targeted delivery and on-demand release of antiCancer and anti-HIV drugs as well as nano-stimulators for non-invasive treatment of patients with central nervous system (CNS) disorders, such as Parkinson's Disease, Alzheimer's Disease, and other dementia (97). Further, this intrinsic coupling between electric and magnetic fields within MENs provides molecular composition specificity that can enable an entirely new dimension even to the conventional diagnostic methods, such as MRI and PET-CT. In addition, the specificity allows assigning unique "passport" features to small groups of nanoparticles thus pushing the real-time health monitoring 
capability to a new level. Finally, because the coupling between magnetic and electric fields is achieved at the intrinsic level, ideally the described field-controlled PNM functions (delivery, drug release, CNS stimulation, diagnostics) can be accomplished with desirable $(\sim 100 \%)$ efficacy and without any destructive heat dissipation (with no need to use high-power electronics as with the conventional NPs).

This dissertation presents the detailed fabrication protocols and characterization procedures for MENs that could be used for energy-efficient field-controlled targeted drug delivery and on-demand release with no heat dissipation and unprecedented high efficacy. As examples, we discuss HIV and Ovarian Cancer models (In chapter 2, 3, and 4). Moreover, we also presented the potential applications of MENs in restoring the neuronal activity in the brain and to function, as contrasting agents (for MRI and MNI modalities) to capture molecular information of the surrounding tissue/microenvironment (In chapter 5 and 6). 
Chapter 2: Magneto-electric Nanoparticles for Eradicating HIV from CNS 


\subsection{Introduction}

Although highly active anti-retroviral therapy (HAART) has resulted in remarkable decline in the morbidity and mortality in AIDS patients, zero or inadequately low delivery of anti-retroviral (ARV) drugs across the blood-brain barrier (BBB) and into the brain and other tissue organs results in virus persistence (98-100). Hence, the elimination of HIV-1 reservoirs still remains a formidable task $(101,102)$. In recent years, use of nanotechnology in medicine has shown exciting prospect for the development of novel remotely controlled drug delivery systems $(103,104)$. Besides magnetic nanoparticles (MNs) (105-107), other delivery systems rely on using thermally responsive polymers, optically (UV, Visible-Wavelength, and IR) and acoustically activated nanostructures, liposomes, electrochemical processes, and others. An extensive review of these and other active triggering approaches was presented in the article by Timko et al (94). The unique advantages of magnetically forced triggering place this approach in a class of its own. In this case, the speed of delivery is determined by the external magnetic field. For instance, as it is related to the delivery across the BBB (108), this approach provides a way to deliver drugs sufficiently fast to avoid their engulfing by the reticuloendothelial system (RES). Nucleotide reverse transcriptase inhibitor (NRTI) 3'-azido-3'-deoxythymidine-5'-triphosphate (AZTTP) is among the most challenging antiretroviral (ARV) drugs to deliver across the BBB (109). Previously we have shown that MNs tagged with AZTTP transmigrated across the BBB by application of an external magnetic field without affecting the integrity of the BBB and the transmigrated AZTTP demonstrated significant inhibition of HIV-1 p24 antigen production in an in-vitro infection model system compared to the free AZTTP (95). 
However, this MN-based delivery suffers from the lack of certainty of drug release from the carrier if and when the nano-carrier reaches the target. The current presumptive mechanisms of drug delivery depend on manually uncontrollable cellular phenomena, such as exocytosis of drug containing intracellular vesicle, intracellular $\mathrm{Ca} 2+$ concentrations and pathology-specific responses (change in $\mathrm{pH}$, temperature, etc.) (96). As a result, to obtain a relatively small physiologic change and ensure the release of the drug, the binding force between the nano-carrier and the drug must be maintained relatively weak. Consequently, more than $99 \%$ of the drug/carriers are deposited in liver, lungs and other lymphoid organs before they reach the target. Therefore, an approach for finely controlled and enhanced release of AZTTP in sufficient therapeutic levels in in the brain or in other target organs is still sought after, as it is critical for the complete eradication of HIV.

We present a study to demonstrate that dissipation-free, energy-efficient and low-field on-demand drug release can be achieved if the conventional MN carriers are replaced by magneto-electric (ME) nanoparticles (MENs) (110). ME materials represent a relatively recently introduced class of multi-functional nanostructures in which magnetic and electric fields can be strongly coupled at body temperature (64). Similar to the MNs, MENs can be designed to have adequately high magnetic moments and therefore, also can be used for targeted delivery by applying remote direct-current (d.c.) magnetic fields. However, due to their non-zero magneto-electricity, unlike the traditional MNs, MENs offer an additional feature that can enable a new dissipation-free mechanism to force a 
high-efficacy externally controlled drug release process at the sub-cellular level using remote low-energy d.c. and/or alternating-current (a.c.) magnetic fields.

An exaggerated illustration in Figure 7 explains the concept of the field-triggered ondemand drug release from MENs. To simplify the description, we use an example with a remote magnetic field in one specific direction, e.g. along X-axis, with respect to the MEN-drug nano-complex. The original (zero-field) ionic bond, with charge $Q_{i o n i c}$ of the nanoparticle, is schematically shown in Figure 1a. AZTTP molecules (typically interconnected in chains) surround each MEN in a symmetric fashion. As shown in Figure 1b, as a non-zero magnetic field is applied, a non-zero electric dipole moment is formed in the nanoparticle due to the non-zero ME effect. For simplicity assuming an isotropic model, the triggered dipole moment $\Delta P=\alpha H$, where $\alpha$ is the $1^{\text {st }}$ order ME coefficient and $H$ is the magnetic field. The amplitude of the dipole charge surface density on each side of the nanoparticle along the field would be of the order of $\sigma_{M E} \sim \pm \alpha H$, where "positive" and "negative" signs are applied to the opposite sides of the dipole, respectively. The dipole moment breaks the original symmetry of the charge in the MEN shell. Consequently, as the magnitude of the magnetic field is further increased above the threshold value at which the dipole charge density on the "negative" side becomes comparable to the positive ionic charge density in the shell, $\sigma_{M E} \sim Q_{i o n i c} / \pi \mathrm{d}^{2}$, i.e. $H_{t h} \sim$ $Q_{\text {ionic }} / \pi \mathrm{d}^{2} \alpha$, where $d$ is the diameter of the MEN, the bond in this direction along the $\mathrm{X}$ axis will be broken while the opposite bond will be further strengthened, as illustrated in Figure 1c. By symmetry, to break the bond in the opposite direction, the field sequence should be repeated in the reverse direction, as illustrated in figures 1D-E. This simplified 
scenario doesn't take into account the randomness of the orientations of the nanoformulations. Ideally, applying an AC magnetic field that equivalently sweeps all bond orientations will create a more uniform and efficient bond-breaking process over the surface of the nanoformulation and thus enhance the drug release efficacy. In the next generation of the technology, this goal can be achieved by using a spatially rotating field, which in turn can be accomplished, for example, by using an array of coils that generate AC fields with non-zero phase shifts with respect to each other.

a. $\mathrm{H}=0 \mathrm{Oe}$

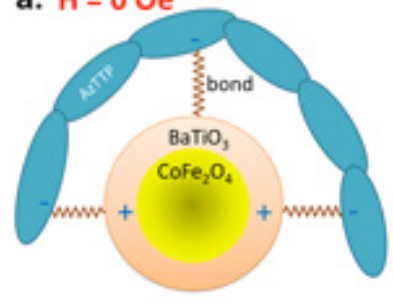

d. $-10<\mathrm{H}<0$ Oe
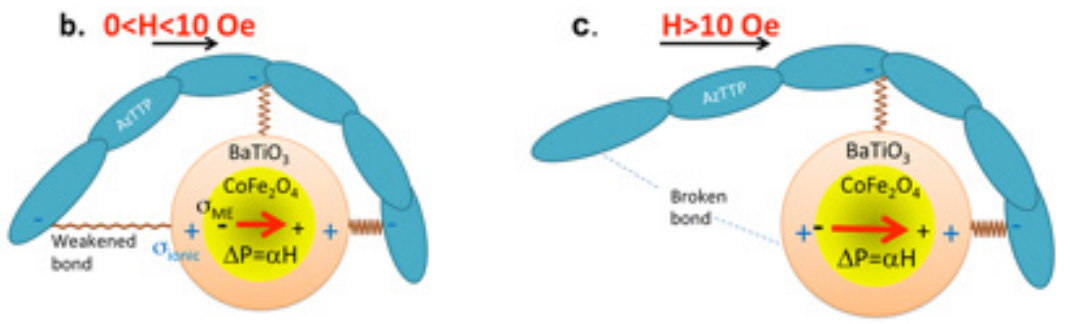

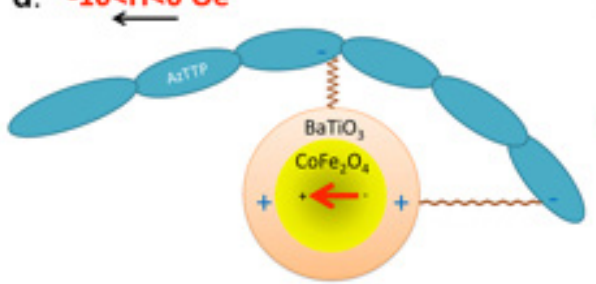

e. $\mathrm{H}<-10 \mathrm{Oe}$

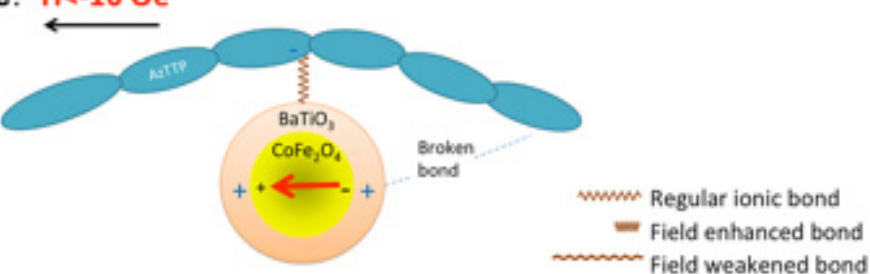

Figure 7: Illustration of the underlying physics of the a.c.-field-triggered release. A simplified (one-directional) illustration of the concept of on-demand drug (AZTTP) release by MENs stimulated by a uniform a.c. magnetic field in X-direction: (a) At zero field, only the ionic charge is present in the MEN shell; (b) An additional dipole moment (proportional to the magnetic field) breaks the original symmetry of the charge distribution in the shell; (c) As the field is increased above the threshold value $\left(\sigma_{i o n i c} \sim \sigma_{M E}\right)$, the bond on one side is broken. (d) and (e) The field is reversed to break the bond on the opposite side of the nanoparticle. The red arrows show the electric dipole due to the ME effect. In practice, due to the random configurations of nanoformulations with respect to the field, the effect is present along every central bond orientation.

In this study, we verified the hypothesis by demonstrating the on-demand release of AZTTP from 30-nm $\mathrm{CoFe}_{2} \mathrm{O}_{4} @ \mathrm{BaTiO}_{3} \mathrm{MENs}$ by applying low a.c. and d.c. magnetic fields. We showed that a 44-Oe a.c. field at $1000 \mathrm{~Hz}$ was sufficient to trigger over $89 \%$ 
release. Further, to support the dissipation-free release model, we could use a 66-Oe d.c. field to trigger a comparable release level.

\subsection{Experimental methods}

\subsubsection{Preparation of CoFe2O4-BaTiO3 coreshell MENs}

$\mathrm{CoFe}_{2} \mathrm{O}_{4}$ nanoparticles were prepared by hydrothermal method. In this method, $15 \mathrm{ml}$ of aqueous mixture of $0.058 \mathrm{~g}$ of $\mathrm{Co}\left(\mathrm{NO}_{3}\right)_{2} \cdot 6 \mathrm{H}_{2} 0,0.16 \mathrm{~g}$ of $\mathrm{Fe}\left(\mathrm{NO}_{3}\right)_{3} \cdot 9 \mathrm{H}_{2} \mathrm{O}$ and $0.2 \mathrm{~g}$ of polyvinylpyrrolidone is dissolved in $5 \mathrm{ml}$ of aqueous $0.9 \mathrm{~g}$ of sodium borohydride at $120 \mathrm{C}$ for 12 hours. Next, precursor solution of $\mathrm{BaTiO}_{3}$ was prepared by mixing $30 \mathrm{ml}$ of aqueous $0.029 \mathrm{~g}$ of $\mathrm{BaCO}_{3}$ and $0.1 \mathrm{~g}$ of citric acid with $30 \mathrm{ml}$ of ethonalic solution of 0.048ml titanium isopropoxide and 1g of citric acid. $\mathrm{CoFe}_{2} \mathrm{O}_{4} @ \mathrm{BaTiO}_{3}$ coreshell MENs are prepared by dispersing $0.1 \mathrm{~g}$ of $\mathrm{CoFe}_{2} \mathrm{O}_{4}$ nanoparticles in the precursor solution. The mixture was sonicated for 2 hours. Well-dispersed mixture was dried at $60^{\circ} \mathrm{C}$ for overnight while stirring continuously. Later the mixture was subjected to calcination at $780^{\circ} \mathrm{C}$ for 5 hours. By reducing the cooling rate (controlled by furnace CMF 1100) from above 52 to below $14 \mathrm{C} / \mathrm{min}$, the average diameter of MENs could be controlled from below $25 \mathrm{~nm}$ to over $100 \mathrm{~nm}$, respectively, with an adequate size distribution of less than $30 \%$. The particle size distribution was measured by a Zetasizer Nano series via the standard dynamic light scattering (DLS) approach. For the $25-\mathrm{nm}$ process $(14 \mathrm{C} / \mathrm{min}$ cooling rate), the average size distribution ranged from approximately 19 to $31 \mathrm{~nm}$. 


\subsubsection{Conjugating AZTTP with MENs (AZTTP-MEN Nanoformulations)}

$10 \mu 1$ of AZTTP drug $(10 \mathrm{mM}$, concentration) was added to the solution of $190 \mu \mathrm{l}$ of TE buffer and $50 \mu 1$ of $\mathrm{CoFe} 2 \mathrm{O} 4-\mathrm{BaTiO} 3$ coreshell MENs $(5 \mathrm{mg} / \mathrm{ml})$. Then, the solution was incubated for $2 \mathrm{hrs}$ and $3 \mathrm{hrs}$. After the incubation, the solution was subjected to the magnetic field in-order to precipitate the MENs (conjugated with AZTTP). After the supernatant was isolated, its absorption was measured at $267 \mathrm{~nm}$ using spectrophotometer Agilent Cary 100.

\subsubsection{Drug loading percentage}

Drug loading percentage $=($ Absorbance of total amount of drug used - Absorbance of drug used in supernatant after incubating the drug and the MEN for a specific incubation time) x $100 \%$ :

After $2 \mathrm{hr}$ incubation, the percentage of the drug in the supernatant was $\sim 89 \%$, which corresponded to approximately $11 \%$ (100-89) of the drug bound to the MENs. After $3 \mathrm{hr}$ incubation, the percentage of the drug in the supernatant was $\sim 76 \%$, which corresponded to approximately $24 \%$ (100-76) of the drug bound to the MENs.

Drug release percentage: drug release percentage $=($ absorbance of supernatant after magnetic field treatment)/(absorbance of supernatant after incubating the particles with drug) $\times 100 \%$. The absorbance signal was measured at the maximum wavelength of 267 nm. 


\subsubsection{Magnetic Field Treatment}

To eliminate any potential residual unbounded drug, after isolating the supernatant, the precipitate of the MENs conjugated with the drug was washed once with the TE buffer. Then, the drug-conjugated particles (AZTTP-MEN nanoformulations) were re-dispersed in $190 \mu 1$ of the TE buffer, and subjected to a magnetic field of varying field strength and frequency (shown in Figure 8). Finally, the solution was precipitated to pipette out the supernatant for further absorption measurements.

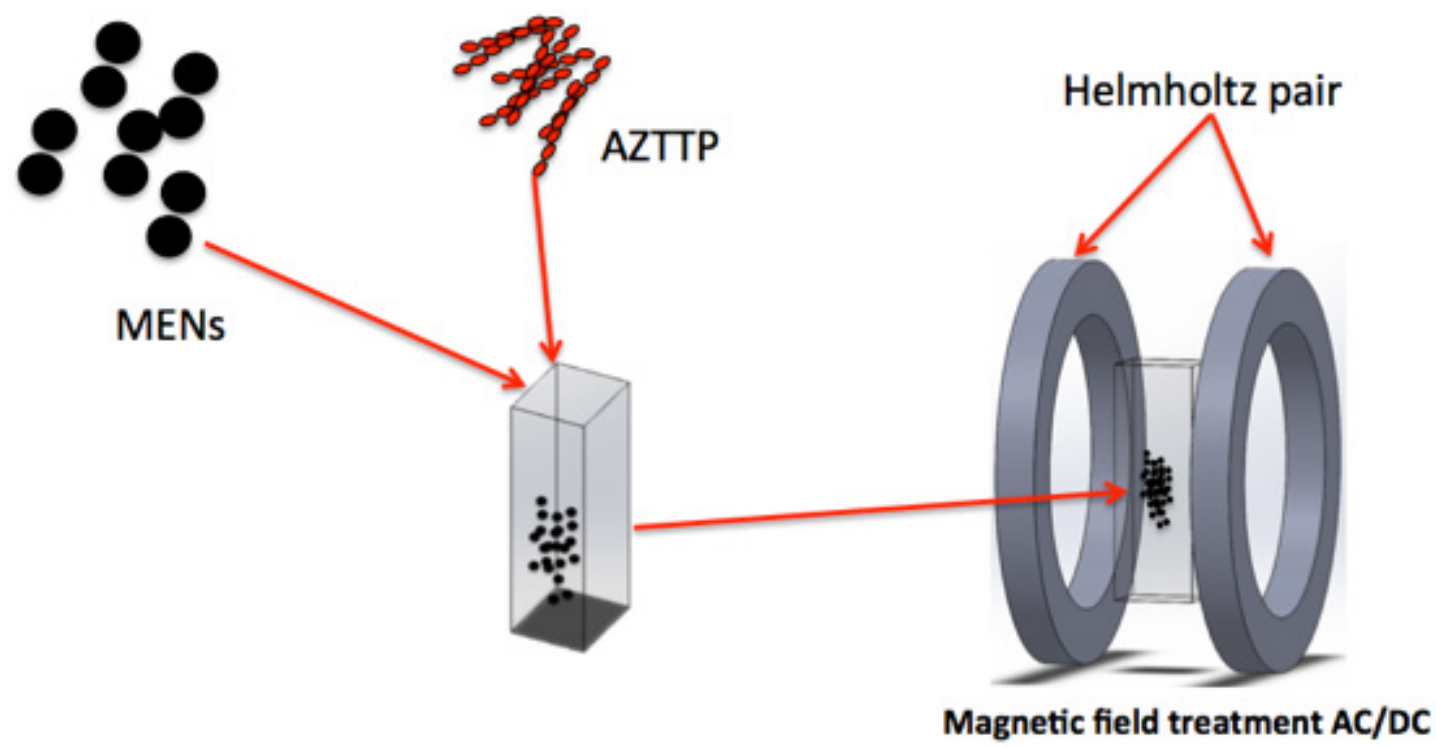

Figure 8: Magnetic fields treatment setup. Drug loaded MENs are treated in the uniform magnetic fields using a pair of Helmholtz coils. 


\subsubsection{Surface Charge of MENs and AZTTP Molecules}

Because of the triphosphate functional groups, in the chemical (covalent and/or ionic) bond between the MENs and the AZTTP molecular cluster chains, the $\mathrm{CoFe}_{2} \mathrm{O}_{4}-\mathrm{BaTiO}_{3}$ MENs and the AZTTP chains were positively and negatively charged, respectively.

\subsubsection{Mass spectrometry analysis of AZTTP molecules, AZTTP bound to MEN} before and after the a.c.-triggered release.

After the a.c.-triggered release by MENs, we conducted a mass spectrometry analysis. The analysis included a full scan qualitative identification of the target analyte, a chromatographic separation and an assessment of the presence of the drug in the composite material (AZTTP-MEN nanoformulations) using multiple reaction monitoring (MRM) for appropriate transitions. Different mixes were contained in Eppendorf vials. Using a $100-\mu \mathrm{L}$ micropipette, $100 \mu \mathrm{L}$ of each vial was transferred to a new disposable polypropylene 96-well plate (Part \# 5042-1386). The vials' contents were subjected to a flow injection analysis (FIA) using electrospray ionization with Jet Stream Technology in both positive and negative mode to assess the presence of the AZTTP drug. The measurements were conducted with mass spectrometer Agilent LC/MS Triple quadruple G6460 LC-QQQ. The ionization conditions are presented in Table 2. Figure 9 shows the number of counts versus the mass-to-charge ratio for the three key stages of the release process, (top) standard AZTTP, (middle) AZTTP bound to MENs, and (bottom) AZTTP and MEN after the a.c.-triggered release of the drug load, respectively. The molecular weight of the AZTTP molecule is approximately 507. 
Table 2: Mass spectroscopy instrumental parameters used for LC/MSD analyses (general screening method).

\begin{tabular}{|l|c|}
\hline Source Parameter & Value \\
\hline Drying Gas Temp & $325^{\circ} \mathrm{C}$ \\
\hline Gas Flow & $10 \mathrm{psi}$ \\
\hline Nebulizer & $30 \mathrm{psi}$ \\
\hline Sheath gas heater & $375^{\circ} \mathrm{C}$ \\
\hline Sheath Gas Flow (1/min) & 11 \\
\hline Nozzle Voltage & $0 \mathrm{~V}$ \\
\hline Capillary Voltage $($ Vcap) & $3500 \mathrm{~V}$ \\
\hline Scan Parameters & $50-1000$ \\
\hline Mass Range & $350 \mathrm{~ms}$ \\
\hline Scan time & $135 \mathrm{~V}$ \\
\hline Fragmentor voltage & $4 \mathrm{~V}$ \\
\hline Cell Acelerator voltage & Negative \\
\hline Polarity & \\
\hline
\end{tabular}
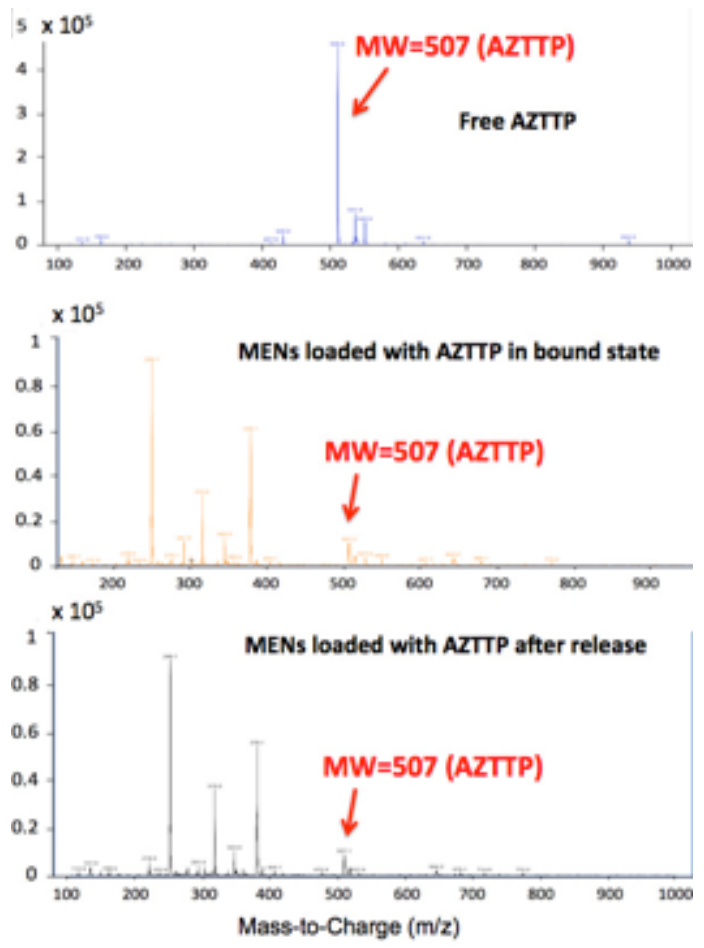

Figure 9: Mass spectrometry analysis: the number of counts versus the mass-to-charge ratio for (top) the free AZTTP, (middle) AZTTP and MENs in the bound state, (bottom) AZTTP and MEN after the a.c. release. 


\subsubsection{Preparation of samples for AFM and FTIR imaging}

$10 \mu 1$ of AZTTP drug $(10 \mathrm{mM}$, concentration) was added to the solution of $190 \mu 1$ of the TE buffer and $50 \mu 1$ of $\mathrm{CoFe}_{2} \mathrm{O}_{4}-\mathrm{BaTiO}_{3}$ coreshell MENs $(5 \mathrm{mg} / \mathrm{ml})$. Then, the solution was incubated for $3 \mathrm{hrs}$. After the incubation, one drop of solution was air dried on to a precleaned Si-wafer. Once dried AFM imaging (topography and phase contrast) were performed using Nanoscope IIIa Multimode:

--For AZTTP: the above solution without MENs.

--For MENs: the above solution without AZTTP.

Note: for FTIR measurements, the sample preparation procedures were similar to the one used for AFM.

\subsubsection{In-vitro BBB Model}

In-vitro BBB model was prepared according to the procedure described elsewhere (111). The BBB model consisted of two compartments (upper and lower), which were separated by the polyethylene terephthalate (PET) membrane of a $3.0 \mu \mathrm{m}$ pore size (Becton Dickinson Labware, NJ. USA). In a 24-well cell culture inserts, $2 \times 10^{5}$ human brain microvascular endothelial cells (HBMEC) were grown to confluence on the upper-side of the insert and the same amount of human astrocytes were grown on the underside. BBB cell culture was incubated for 4 days in-order to reach incubation confluence at $37^{\circ} \mathrm{C}$ and 5\% $\mathrm{CO}_{2}$. Transendothelial electrical resistance (TEER) was used to measure the intactness of the BBB. The average TEER value for individual BBB was about 150 to $200 \mathrm{ohms} / \mathrm{cm} 2$, which is consistent with formation of BBB 


\subsection{9. p24 Assay to confirm the integrity of AZTTP drug}

Normal peripheral blood mononuclear cells (PBMCs) $\left(10 \times 10^{6}\right.$ cells $)$ were infected with native HIV-1 $1_{\mathrm{Ba}-\mathrm{L}}(\mathrm{NIH}$ AIDS Research and Reference Reagent Program catalog no. 510) at a concentration of $2 \mathrm{ng} / \mathrm{ml}$ cells for 2 hours, washed with phosphate-buffered saline (PBS) (GIBCO-BRL, Grand Island, NY) and returned to culture with and without fresh (new) or MEN-released AZTTP at equimolar concentrations (200 nM). The culture supernatants were quantitated for p24 antigen using a p24 ELISA kit (ZeptoMetrix, Buffalo, NY) on the fifth day of infection. While the control sample showed the p24 concentration of approximately $33050 \mathrm{pg} / \mathrm{ml}$, the use of fresh and MENs-released AZTTP drugs resulted in the concentration values of approximately 1300 and 1800 $\mathrm{pg} / \mathrm{ml}$, respectively (Figure 10). Therefore, considering the standard deviation of approximately $300 \mathrm{pg} / \mathrm{ml}$, the two drug forms demonstrated equal p24 inhibition efficacy.

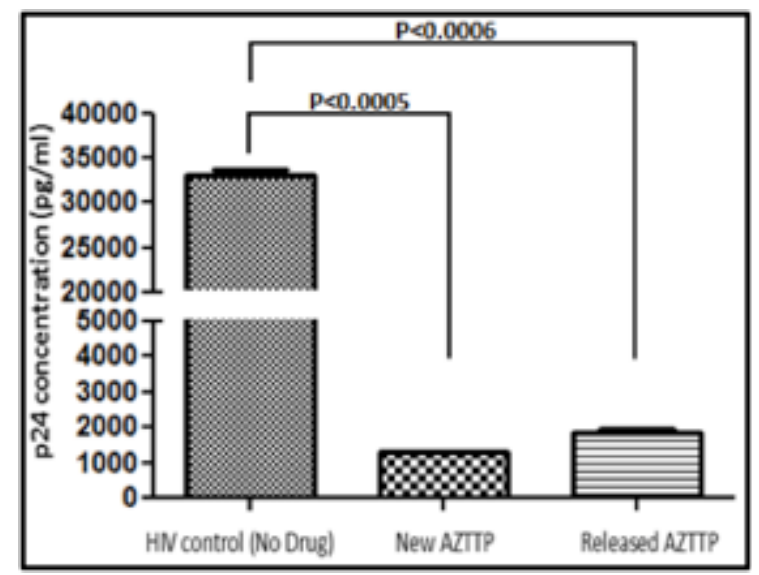

Figure 10: P24 study to prove that the on-demand release does not affect the inhibition efficacy of the AZZTP drug. The $\mathbf{P 2 4}$ concentration is in $\mathrm{pg} / \mathrm{ml}$ (units). 


\subsubsection{In-vitro cytotoxicity assay}

To understand the in-vitro cytotoxicity of MENs $\left(\mathrm{CoFe}_{2} \mathrm{O}_{4} @ \mathrm{BaTiO}_{3}\right)$, we performed XTT (sodium 2,3,-bis(2-methoxy-4-nitro-5-sulfophenyl)-5-[(phenylamino)-carbonyl]2H-tetrazolium inner salt) assay according to the manufacturer's protocol (ATCC). XTT is a quantitative colorimetric assay, which is based on the formation of an orange colored formazan dye by viable cells from the cleavage of XTT tetrazolium salts. The assay was performed on human astrocyte (HA) cells for neuronal cytotoxicity and on peripheral blood mononuclear cells (PBMc) for peripheral cytotoxicity. In this assay, desired cells were seeded in a 96-well cell culture plate at a $1 \times 10^{5}$ cells/well concentration and incubated for 48 hours. Note: performing a pre-assay optimization protocol standardized the cell concentration and incubation time (show in Figure 11). Following the incubation, the cell culture medium was replaced by a medium containing MENs at a differential concentration of 0 -to- $50 \mu \mathrm{g} / \mathrm{ml}$. The cells were incubated in the MEN solution for 24 hours. Once, the incubation period was completed the cells were washed with PBS buffer and later supplemented with a fresh medium. Cell viability test was performed by XTT test kit, as supplied by (ATCC) for 6 hours. The experiments that were performed in triplicate indicated no significant toxicity at a concentration of less than $50 \mu \mathrm{g} / \mathrm{ml}$ (shown in Table 3). 


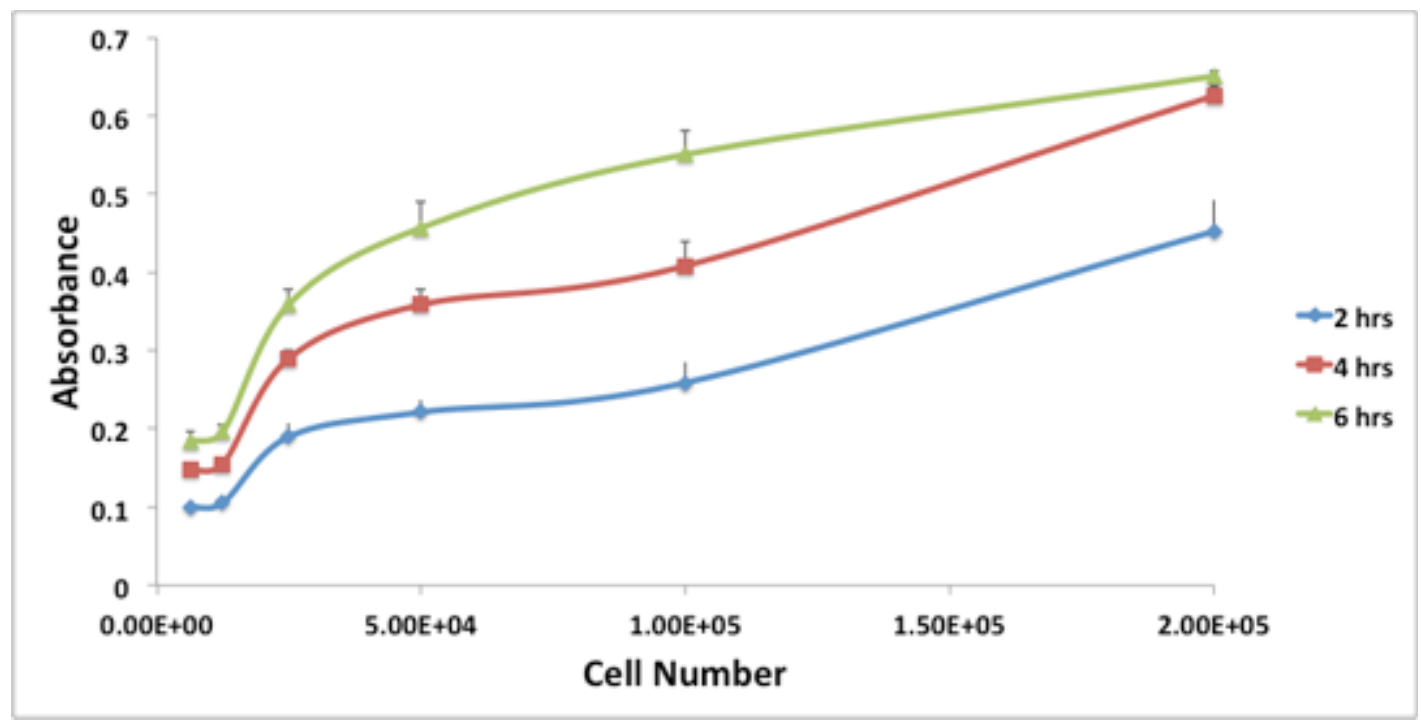

Figure 11: XTT assay optimization assay results for human astrocyte cells for different treatment times $(2,4$, and 6 hrs.).

Table 3: Cell viability test (XTT) to illustrate non-toxicity of MENs at different concentrations.

\begin{tabular}{|c|c|c|}
\hline \multirow{2}{*}{$\begin{array}{c}\text { MEN Concentration } \\
(\mu \mathrm{g} / \mathrm{ml})\end{array}$} & \multicolumn{2}{|c|}{ Cell viability percentage for different cell types (\%) } \\
\cline { 2 - 3 } & HA & PBMCs \\
\hline 0 & $100 \pm 0.3$ & $100 \pm 2.7$ \\
\hline 10 & $100.1 \pm 1.2$ & $99.91 \pm 1.1$ \\
\hline 20 & $99.8 \pm 3.6$ & $99.94 \pm 0.2$ \\
\hline 30 & $98.3 \pm 2.1$ & $99.76 \pm 2.7$ \\
\hline 40 & $96.1 \pm 3.5$ & $98.78 \pm 1.5$ \\
\hline 50 & $95.9 \pm 2.6$ & $98.38 \pm 0.2$ \\
\hline
\end{tabular}




\subsection{Results}

We used atomic force microscopy (AFM) in conjunction with UV-spectrophotometry, Fourier Transform Infra-Red (FTIR) analysis, and mass spectroscopy to directly trace the kinetics of the drug release process at different stages of the release under the influence of remote d.c. and a.c. magnetic fields. The three key stages included (i) the initial state with separate MEN carriers and AZTTP molecules, (ii) the loaded state in which MENdrug nano-formulations are formed, and (iii) the final state after the a.c.-field-forced separation of AZTTP and MENs, i.e. after the on-demand drug release. In-vitro experiments on HIV-infected human cells were conducted to demonstrate the structural and functional integrity of AZTTP after this physical release process. Below we present the key results of this experimental study. The experiments are described in more detail.

\subsubsection{Transmission electron microscopy study of MENS}

In the experiments described below, for the role of MENs we used nanoparticles made of the popular core-shell composition $\mathrm{CoFe}_{2} \mathrm{O}_{4} @ \mathrm{BaTiO}_{3}$, in which the relatively high moment $\mathrm{CoFe}_{2} \mathrm{O}_{4}$ core was used to enhance the magnetic moment (112). In general, nanoparticles as small as 5-nm in diameter can be fabricated with physical methods, such as ion beam proximity lithography or imprint lithography (113). In this study, considering the novelty of the approach, we focused on the main discovery of using MENs for on-demand drug release rather than on the development of scaling approaches. The default measurements were conducted with 30-nm MENs. A typical transmission electron microscopy (TEM) image of the fabricated MENs, with clearly visible core-shell 
structures, is shown in Figure 12A. The composition of the MENs was confirmed through energy-dispersive spectroscopy (EDS), as shown in Figure 12B. The ME coefficient for the nanoparticles was measured via point I-V methods in the presence of a field to be the order of $100 \mathrm{~V} \mathrm{~cm}^{-1} \mathrm{Oe}^{-1}$ using an approach described in our previous publication (114).
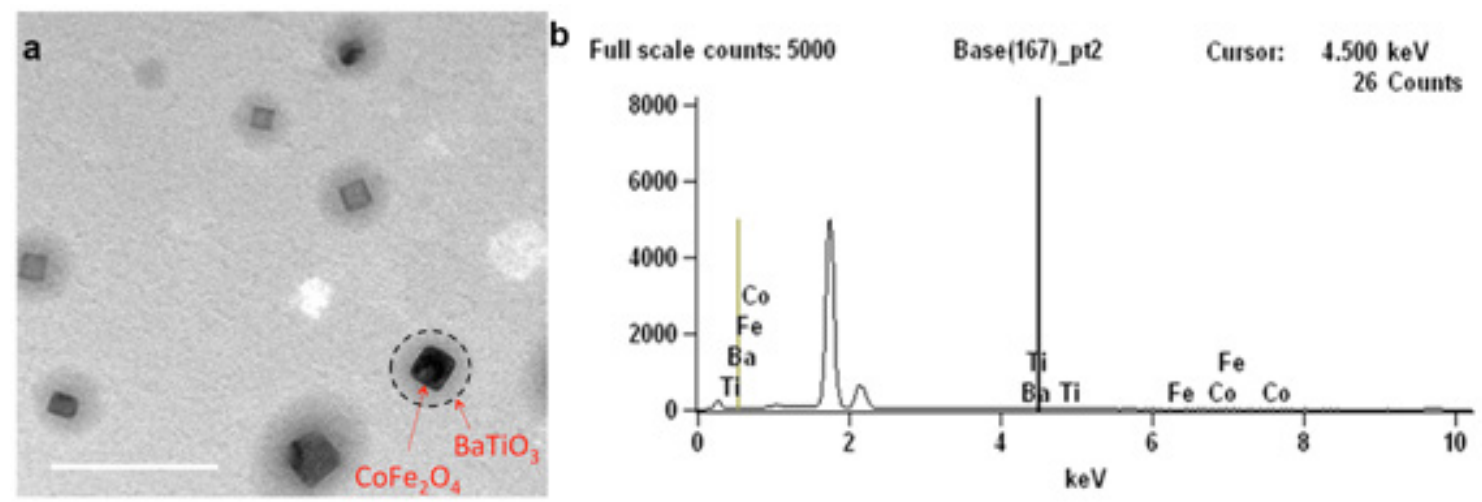

Figure 12: Transmission electron microscopy analysis of MENs. (a) A transmission electron microscopy (TEM) image of MENs. The core-shell structure of MEN is highlighted. The scale bar is $100 \mathrm{~nm}$. (b) An energydispersive spectroscopy (EDS) analysis of MENs.

\subsubsection{Spectrophotometry study of the release}

The goal of the first experiment was to measure the amount of the drug (AZTTP) at different stages of the release process using the conventional approach of spectrophotometry by measuring the UV-light absorption at the 267-nm maximum of the drug's absorption spectrum. We could bind approximately $24 \%$ of the drug to the nanoparticles by incubating AZTTP with the MENs in the Tris-EDTA (TE) buffer (pH 7.4) for 3 hours. The amount of the bound drug was determined by estimating the concentration of AZTTP in the unbound fraction (supernatant) of the incubation mixture by spectrophotometry. To apply remote d.c. and a.c. magnetic fields, we used a low- 
energy low-field Helmholtz pair connected to a function generator. The chart in figure 3 summarizes the key results of this experiment, in which we measured the amount of the unbound drug depending on the external field strength and frequency. It can be seen that application of a $44-\mathrm{Oe}$ field at $1000 \mathrm{~Hz}$ results in $89.3 \%$ of the drug being released, while application of a d.c. field of the same amplitude releases only $16.4 \%$ of the drug. The data also confirms that with higher frequency, a lower magnetic field is needed to break the bonds because oscillation of the bonds caused by a higher frequency field facilitates the breaking of the bonds. However, when a strong enough d.c. field ( $>65 \mathrm{Oe})$ is applied, the bond-breaking side of the drug chain can gain enough momentum, causing the chain to break free from the MEN shell even at zero frequency. To fully exploit the potential of the new controlled drug release nanotechnology, it is important to conduct a more detailed pharmacokinetics study.

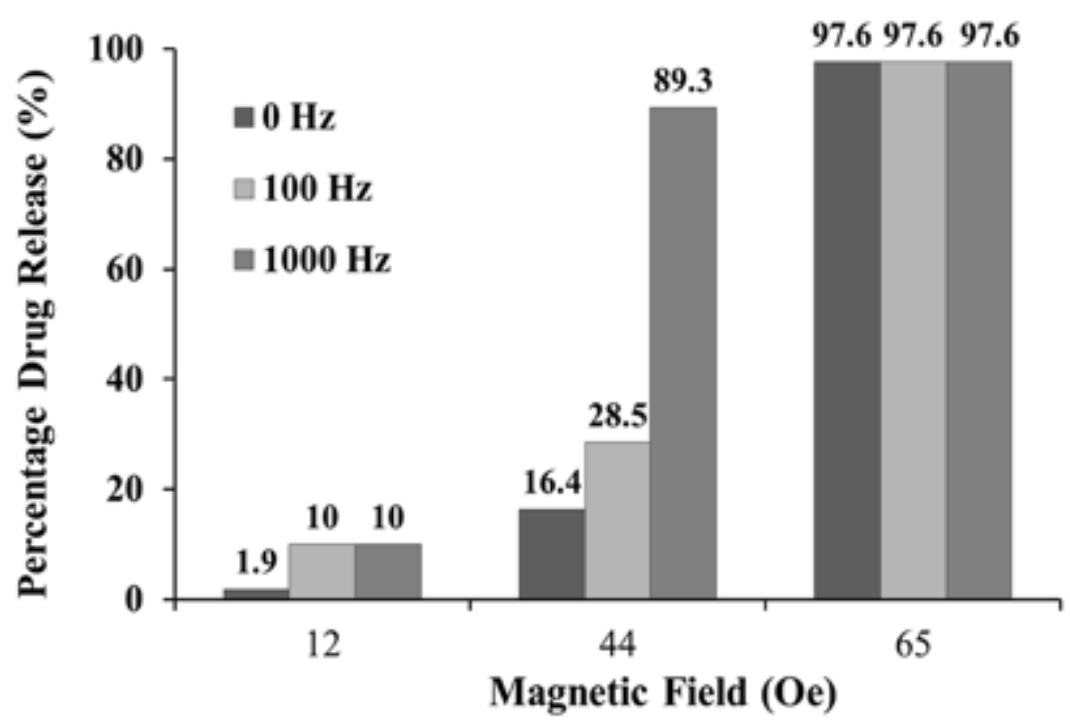

Figure 13: Field strength and frequency dependence of the drug release. Chart showing the release efficacy of AZTTP drug bound to MENs by a remote magnetic field at different amplitudes and frequencies. 


\subsubsection{Pharmacokinetics study}

In this experiment, MENs loaded with AZTTP drug were exposed to an external magnetic field at different strengths $(12 \mathrm{Oe}, 44 \mathrm{Oe}$ and $66 \mathrm{Oe})$ and frequencies $(0 \mathrm{~Hz}, 100$ $\mathrm{Hz}, 1000 \mathrm{~Hz})$ at different treatment durations $(1,5,10,60$, and 120 minutes) in-order to understand the release kinetics. Results are summarized in the 3-D chart in Figure 14. The quantitative values are also presented in Table 4. For every field-frequency combination set we used a fresh solution with AZTTP-loaded MENs.

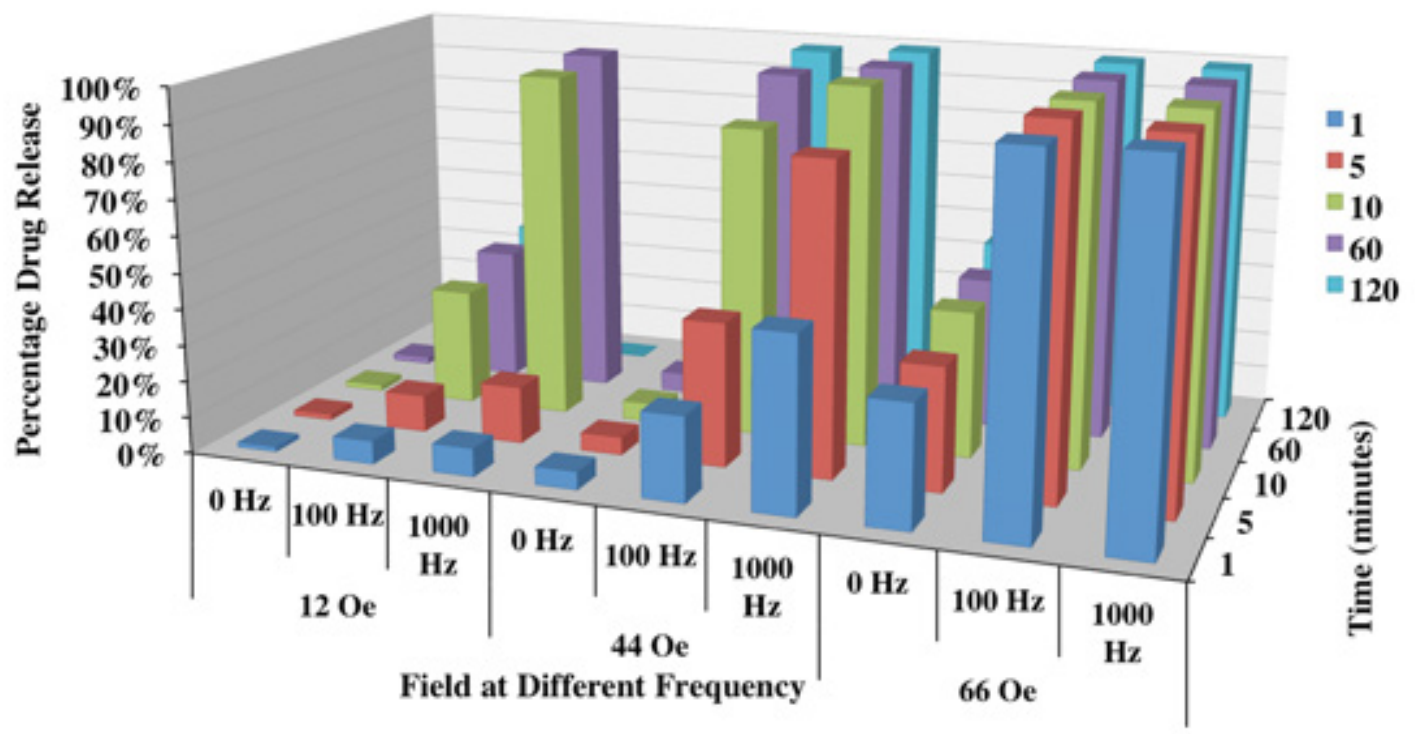

Figure 14: Pharmacokinetics study. Pharmacokinetics Study: 3-D Chart representation of the drug release percentage at various combinations of the field strength $(12,44$, and $66 \mathrm{Oe})$, the frequency $(0,100$, and $1000 \mathrm{~Hz})$, and the treatment duration $(1,5,10,60$, and $120 \mathrm{~min})$. 
Table 4: Drug release percentage at various combinations of field strength, frequency, and treatment duration

\begin{tabular}{|c|c|c|c|c|c|c|}
\hline \multirow{3}{*}{ Field } & \multirow{2}{*}{ Frequency } & \multicolumn{5}{|c|}{$\begin{array}{c}\text { Drug Release Percentage for Different Field Treatment } \\
\text { Durations (minutes) }\end{array}$} \\
\cline { 2 - 7 } & & $\mathbf{1}$ & $\mathbf{5}$ & $\mathbf{1 0}$ & $\mathbf{6 0}$ & $\mathbf{1 2 0}$ \\
\hline \multirow{3}{*}{$\mathbf{1 2}$ Oe } & $0 \mathrm{~Hz}$ & $1.31 \%$ & $1.50 \%$ & $1.52 \%$ & $1.87 \%$ & $1.81 \%$ \\
\cline { 2 - 7 } & $100 \mathrm{~Hz}$ & $6.40 \%$ & $10.14 \%$ & $32.20 \%$ & $36.80 \%$ & $37.89 \%$ \\
\cline { 2 - 7 } & $1000 \mathrm{~Hz}$ & $7.81 \%$ & $15.73 \%$ & $96.20 \%$ & $98.01 \%$ & $98.7 \%$ \\
\hline \multirow{3}{*}{$\mathbf{4 4 ~ O e}$} & $0 \mathrm{~Hz}$ & $5.01 \%$ & $4.90 \%$ & $5.10 \%$ & $5.32 \%$ & $5.41 \%$ \\
\cline { 2 - 7 } & $100 \mathrm{~Hz}$ & $23.30 \%$ & $39.58 \%$ & $85.60 \%$ & $95.66 \%$ & $98.00 \%$ \\
\cline { 2 - 7 } & $1000 \mathrm{~Hz}$ & $47.77 \%$ & $85.33 \%$ & $99.01 \%$ & $99.15 \%$ & $99.31 \%$ \\
\hline \multirow{3}{*}{$\mathbf{6 6}$ Oe } & $0 \mathrm{~Hz}$ & $33.30 \%$ & $33.90 \%$ & $40.23 \%$ & $41.60 \%$ & $44.77 \%$ \\
\cline { 2 - 7 } & $100 \mathrm{~Hz}$ & $98.05 \%$ & $99.21 \%$ & $98.71 \%$ & $99.21 \%$ & $99.27 \%$ \\
\cline { 2 - 7 } & $1000 \mathrm{~Hz}$ & $98.58 \%$ & $97.65 \%$ & $98.37 \%$ & $99.31 \%$ & $99.11 \%$ \\
\hline
\end{tabular}

\subsubsection{Fourier-transform infra-red analysis}

The concept of the drug release by a remote magnetic field was confirmed also through Fourier Transform Infra-Red (FTIR) analysis, as shown for the three key stages of the process kinetics in Figure 15: (top) free MENs and AZTTP before loading, (middle) loaded state: MEN-AZTTP nanoformulations, and (bottom) MENs after the AZTTP release by a remote $44-\mathrm{Oe}$ field at a $100-\mathrm{Hz}$ frequency. Compared to the initial and final unbound states, the loaded state showed almost $30 \%$ weaker absorbance in general and a transformed spectrum in the wavenumber region from 1750 to $1250 \mathrm{~cm}^{-1}$. 


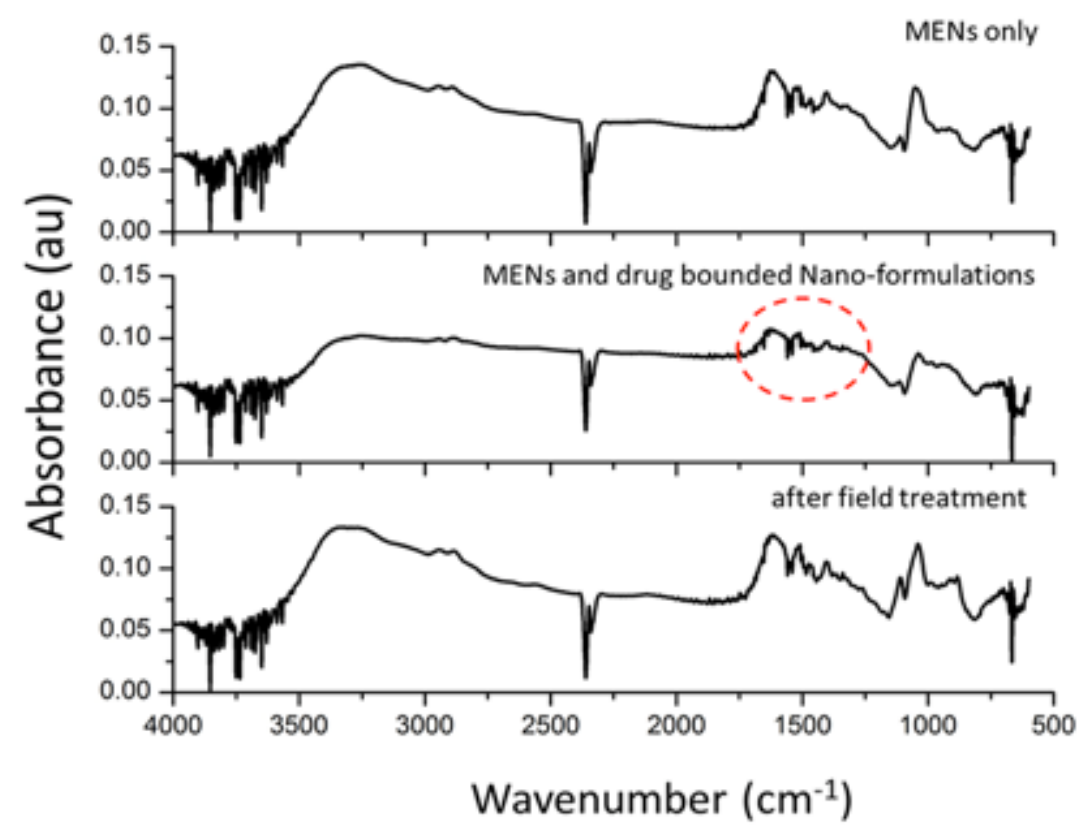

Figure 15: FTIR measurements. FTIR measurements for MEN-AZTTP system at three different stages: (i) (top) MENs only, (ii) AZTTP-loaded MENs, and (iii) MENs after the release by the a.c.-field treatment.

\subsubsection{Atomic force microscopy study of the release kinetics}

To observe the release process at the molecular level, we conducted the following AFM measurements. Figure 16 shows a sequence of AFM images that reflect the following four stages of the release process: free (a) MEN and (b) AZTTP chains, (c) loaded MENAZTTP nanoformulations, and (d) MENs and (e) AZTTP after the release by a 44-Oe a.c. field at $1000 \mathrm{~Hz}$. To obtain images $\mathrm{c}$ and d, the unloaded drug was washed away with the supernatant. One can observe that the MENs and AZTTP chains before the loading step and after the release process look similar. 

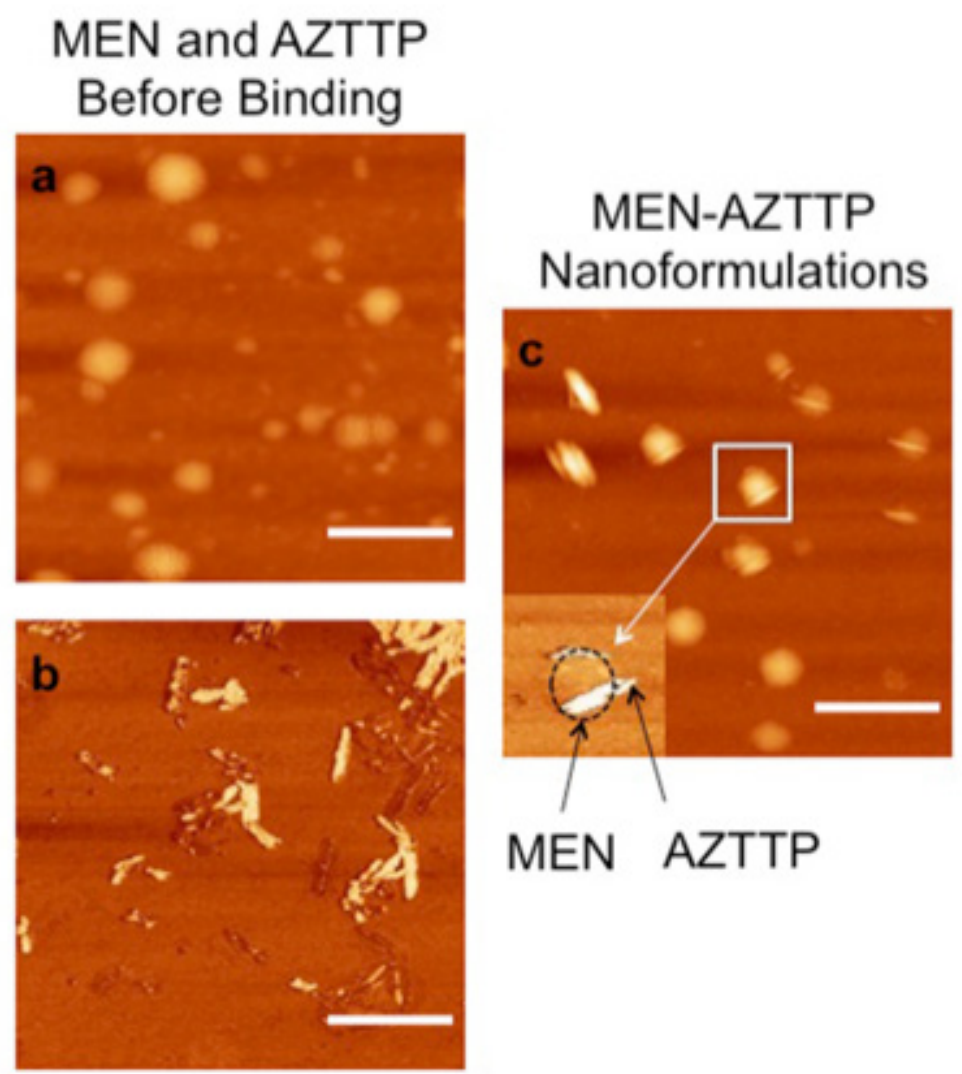

\section{MEN and AZTTP \\ After a.c. Release}
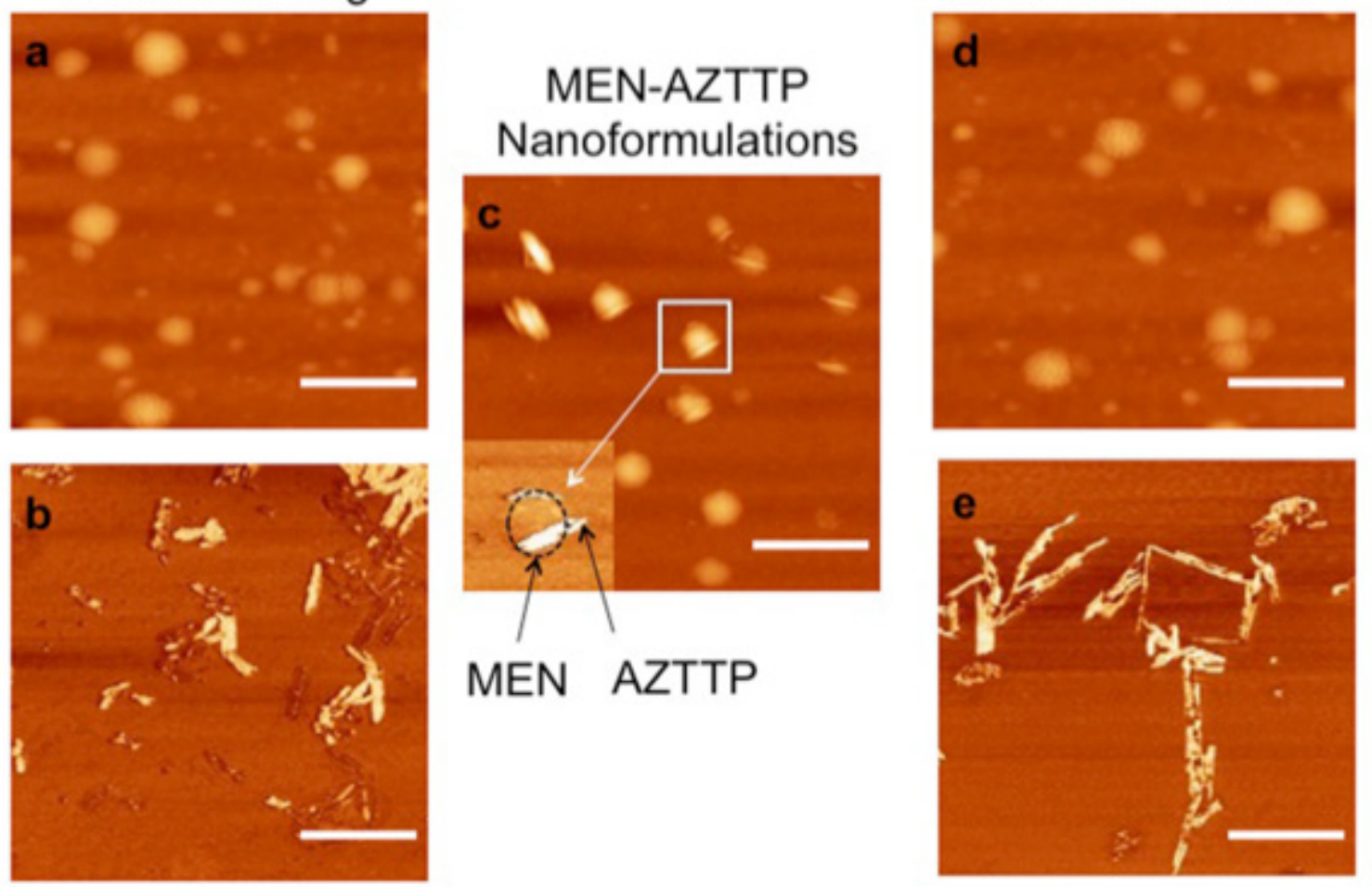

Figure 16: Atomic force microscopy study of the drug release kinetics. AFM images at different stages of the release process: (a) MENs and (b) AZTTP chains before the loading (binding) step, (c) AZTTP-MEN nanoformulations after the loading process, (d) MENs and (e) AZTTP after the drug release by a 44-Oe a.c. field at $1000 \mathrm{~Hz}$. The scale bar is $100 \mathrm{~nm}$.

2.3.6. Field-controlled delivery and drug release by MENs loaded with AZTTP across BBB in in-vitro model.

Translocation experiments were performed on day 5 of BBB cell culture. In-order to achieve the translocation of AZTTP across the BBB, AZTTP were loaded on MENs and subjected to an external magnetic field of 40 Oe (to avoid any unwanted drug release at higher fields) for 3 hours and a gradient of approximately $22 \mathrm{Oe} / \mathrm{cm}$ (to pull the nanoparticles across the BBB) for 6 hours of the incubation period. To apply a field 
normal to the BBB, the field coils were placed below the cell culture wells carrying the BBB model. Once the incubation was completed, the medium in the bottom chamber was isolated and subjected to the a.c. magnetic field (66 Oe at $100 \mathrm{~Hz}$ field for 5 minutes) to increase the release percentage. After the field treatment, the MENs were isolated via a magnetic separation technique. Once separated, the supernatant was measured for the drug concentration via spectrophotometry. The results indicated that approximately $40 \%$ of the drug was translocated across the $\mathrm{BBB}$, as a result of the process. These results are summarized in Figure 17. To further confirm the structural and functional integrity of the drug after the a.c. release process, we conducted: (i) a mass spectrometry analysis of the drug before and after the release (Figure 9), (ii) an in-vitro experiment to study the p24 inhibition efficacy of the drug before and after the release (Figure 10). Finally, to understand the cytotoxicity of MENs $\left(\mathrm{CoFe}_{2} \mathrm{O}_{4} @ \mathrm{BaTiO}_{3}\right)$, we performed an XTT assay on human astrocyte (HA) cells for neural cytotoxicity and on human peripheral blood mononuclear cells $(\mathrm{PBMc})$ for peripheral cytotoxicity. The results indicated no significant toxicity at a concentration of less than $50 \mu \mathrm{g} / \mathrm{ml}$ (Table 3).

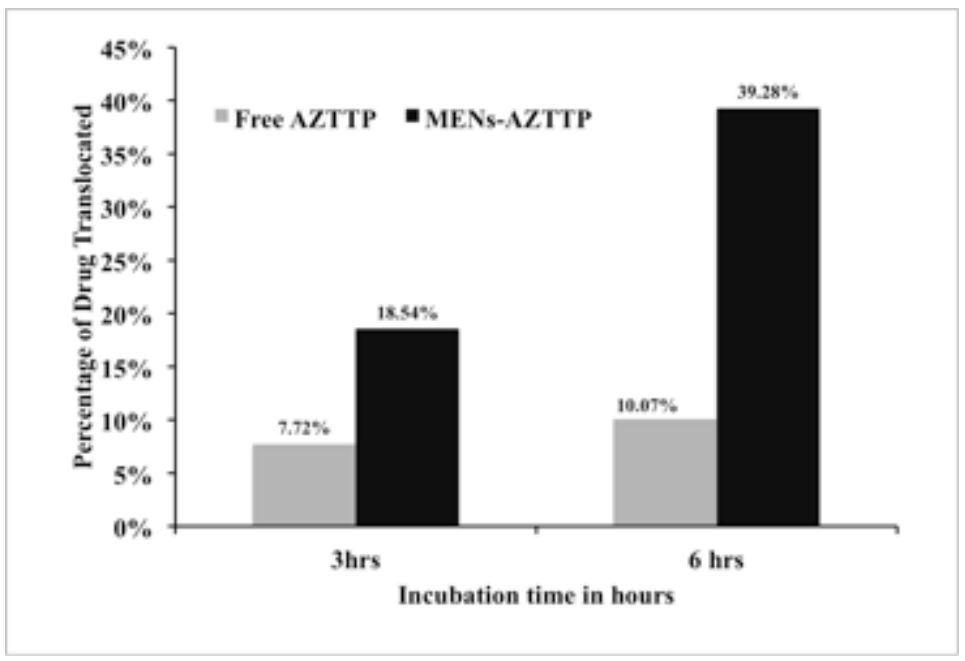

Figure 17: AZTTP-MEN translocation of across BBB. 


\subsection{Discussion}

The spectrophotometry experimental results (Figure 13) indicate the threshold value, necessary for the field to start the release of the AZTTP drugs by the MENs, to be of the order of 10 Oe, even though only $10 \%$ of the drug was released at such a low field value. Using the simplified expression we derived above for the release threshold field $\left(H_{t h} \sim\right.$ $\left.Q_{\text {ionic }} / \pi \mathrm{d}^{2} \alpha\right)$ and assuming the nanoparticle diameter $d=30 \mathrm{~nm}$ and the ME coefficient $\alpha$ $=100 \mathrm{~V} \mathrm{~cm}^{-1} \mathrm{Oe}^{-1}$, we can evaluate the value for the effective charge, $Q_{\text {ionic }}$ (responsible for the ionic/covalent bond between the MEN and AZTTP cluster), to be approximately

$10^{-17} \mathrm{C}$. The estimate seems reasonable and indicates that approximately a few hundred electrons are involved in the bond formation. Further, in agreement with the original hypothesis, the experiment also proves that the a.c. field provides a substantially more enhanced release compared to that of the d.c. field. Indeed, at 12-Oe field amplitude, d.c. and a.c. $(100 \mathrm{~Hz})$ fields results in 1.9 and 10\% released drug percentage, respectively. To achieve almost full $(89.3 \%)$ drug release, a field of 44 Oe at a frequency of $1000 \mathrm{~Hz}$ is required. The effect of the a.c.-field control is significant in this case. For comparison, with a field of $44 \mathrm{Oe}$ at the frequency of $100 \mathrm{~Hz}$, the released amount was $28.5 \%$ while for the d.c. case at the same field amplitude was only $16.4 \%$. The experiment indicates that such nanoformulations can be directed to the targeted cells via a d.c. magnetic field with a spatial gradient while the drug can be effectively released on demand via an external a.c. magnetic field at $1000 \mathrm{~Hz}$. Note that the highest applied field of $65 \mathrm{Oe}$ immediately saturated the system resulting in an almost full drug release even in the d.c. case. According to our hypothesis, the magnetic-field-triggered large electric force that gave enough momentum to break the bond can explain the latter. The results of the more 
detailed pharmacokinetics study (Figure 14) also indicate the presence of a certain threshold field that needs to be applied to trigger the release. The threshold values at different frequencies reach their saturation value in about 60 minutes of the incubation process. Again, this threshold field exists even in the d.c. case (zero frequency) and can be further reduced in the a.c. case by increasing the frequency of an a.c. field. To separate the d.c. and a.c. functions for drug delivery and release, respectively, it is important to maintain the amplitude of the field below the saturation value, e.g., at 44 Oe. This separation of the d.c.- and a.c.-driven delivery and release functions, respectively, was also confirmed in the in-vitro experiment with a human BBB cell culture (Figure 17). The FTIR results are in agreement with spectrophotometry data (Figure 15). Supporting the above release model, the initial state (before drug loading) and the final state (after the release) FTIR spectra look similar while the altered intermediate state reflects the effect of binding between MENs and AZTTP molecules. The AFM measurements (Figure 16) directly illustrate the physical difference between MEN-AZTTP nanoformulations and the free MENs before loading and after releasing the drugs. In agreement with the described spectrophotometry and FTIR measurements, the AFM analysis also indicates that the MENs return to their original unbound state after the drug release triggered by a.c. magnetic field. In addition, AFM also proves the nanoscale nature of the approach, with the potential to be applied at the sub-cellular level. The in-vitro experiment using human PBMCs infected with HIV-1 Ba-L $_{\text {L }}$ proves the intact integrity of the drug after the a.c.-triggered release (Figure 10). 
The fundamental difference between MNs and MENs is due to the presence in the latter of the quantum-mechanically caused ME effect that enables intrinsic coupling between the magnetic spin and the electric dipole. Consequently, energy-efficient and dissipationfree remote control of the intrinsic charge distribution in the MENs (and consequently, control of the bonding force between the MENs and the drug) can be enabled via application of an external magnetic field. Because of the intrinsic ME effect, even if the drug is strongly bonded to the MEN carriers (as required for high-efficacy delivery), it can be fully released at the target location via application of a local magnetic field with a strength above a certain threshold defined by the ME effect. For comparison, the drug release process using the conventional MNs is not controlled at the same fundamental level but instead, is based on an irreversible energy dissipation process caused by an external a.c. magnetic field. In one $\mathrm{MN}$ implementation, superparamagnetic nanoparticles and drug molecules are incorporated into temperature-sensitive synthetic polymers or other biomaterials that release the drug, as the nanoparticles are heated under the field exposure (115). In other implementations, superparamagnetic nanoparticles can be coated with intermediate linkers (tailored to specific load molecules), embedded in a ferro-gel, or directly connected to the load molecules (116). In either of these conventional cases, the release mechanism with $\mathrm{MNs}$ is based on extrinsic phenomena, e.g. mechanical deformation and/or heat dissipation that affects the release kinetics, that are triggered by a relatively high frequency a.c. field (in the range of hundreds of kilohertz or above) and consume a substantial amount of power (in the kilowatt range). On the contrary, the MEN-triggered release process is achieved at the intrinsic level and does not require any intermediate materials. It is dissipation free and extremely energy efficient. The release 
with MENs can be triggered by an a.c. magnetic field at a relatively low frequency (below $100 \mathrm{~Hz}$ ) and even at a d.c. field provided the field strength is above a certain threshold value, as described below in more detail, with power consumption in the subwatt range.

One can argue that MENs may also experience the potential problem of having a relatively large fraction of the nanoparticles (together or without the drug) trapped in the reticuloendothelial system. One solution to this problem is to apply a "zig-zag" shaped time-varying field profile to move the nanoparticles through the system. Then, besides the force along the main delivery path, there is a significant "jolting" force normal to this direction. This "jolting" force ensures that the nanoparticles don't become trapped in the fibers of the reticular connective tissue. However, application of the strongly inertial "zig-zag" force to the conventional MNs can also result in a significant loss of the drug. On the contrary, with MENs, the physical bond between the drug and the nanoparticle can be engineered to be adequately strong to avoid any loss until a command to release is given via an external field.

\subsection{Conclusion}

As a final conclusion, we would like to emphasize that although MENs indeed have a unique advantage of serving in both roles, (i) as regular MNs for drug delivery (via application of a d.c. remote field with a spatial gradient), and (ii) as drug release nanoscale sites, their capability of an on-demand drug release by application of a low remote magnetic field in an extremely low frequency range is unprecedented and 
therefore is a merit of its own (even without exploiting the drug delivery feature). In general, this physically controlled release method could be complementary to any other drug delivery and tagging mechanism, whether it is physical or chemical. Although AZTTP is used in the experiment, the ability of on-demand drug release by a MEN nanocarrier discovered in this study is also relevant to the treatment of other diseases, such as many CNS diseases, cancer and others where deep-tissue high-efficacy drug delivery at the sub-cellular level is key.

Note: How to cite this chapter: The content of this chapter is published in my Nature Communications article, hence please use this as a reference: Nair, Madhavan, Rakesh Guduru, Ping Liang, Jeongmin Hong, Vidya Sagar, and Sakhrat Khizroev. "Externally controlled on-demand release of anti-HIV drug using magneto-electric nanoparticles as carriers." Nature communications 4 (2013): 1707. 
Chapter 3: Magneto-electric Nanoparticles to Enable Field Controlled Highspecificity Drug Delivery to Eradicate Ovarian Cancer Cells 


\subsection{Introduction}

The development of a technology that is capable of high-specificity targeted delivery of antineoplastic drugs would be a significant breakthrough in cancer in general and ovarian cancer in particular. Although the circulatory system can deliver a drug to every cell in the body, delivering the drug specifically inside the tumor cell past its membrane without affecting the healthy cells remains a challenge (117-119). In ovarian cancer, intraperitoneal (IP) delivery through a surgically implanted catheter has shown improved survival rates. However, catheter complications and toxicity have precluded widespread adoption of this invasive means of delivery (120). Current research attempts to go around these limiting factors by using nanoscale systems. In this case, the drug is administered non-invasively through intravenous (IV) injection $(103,104,121)$. Often, as immunological reagents, monoclonal antibodies are used to recognize the tumor-specific biomarker while the nanoscale control further improves the specificity and targeted drug delivery capability in general. Nonetheless, in spite of the tremendous progress in this field during the last decades, the capability of targeted delivery with adequately high specificity (to tumor cells) remains an important roadblock to finding a cure for cancer. In this paper, we present a study in which we address this challenge through a new physical concept. It exploits (i) the difference in the electric properties of the membrane between the tumor and healthy cells and (ii) the ability of the recently discovered body-temperature magnetoelectric nanoparticles (MENs) to function, as nano-converters of remotely supplied magnetic field energy into the MENs' intrinsic electric field energy $(110,122$, 123). Like the conventional magnetic nanoparticles (MNs), MENs have a non-zero magnetic moment and therefore can be controlled remotely via application of an external 
magnetic field. However, unlike MNs, MENs offer a new far-reaching function, which is a dissipation-free and energy-efficient control of the intrinsic electric fields within the nanoparticles by an external magnetic field. This unprecedented capability is a result of the strong magneto-electric (ME) coupling in this new class of nanostructures even at body temperature. Like the conventional magnetic nanoparticles (MNs), MENs have a non-zero magnetic moment and therefore can be controlled remotely via application of an external magnetic field. However, unlike MNs, MENs offer a new far-reaching function, which is a dissipation-free and energy-efficient control of the intrinsic electric fields within the nanoparticles by an external magnetic field. This unprecedented capability is a result of the strong magneto-electric (ME) coupling in this new class of nanostructures even at body temperature $(64,124-126)$. As a result, MENs introduced in a biological microenvironment act, as localized magnetic-to-electric-field nano-converters that allow remote control and generation of the electric signals that underlie the intrinsic molecular interactions. Recently, we exploited this capability: (i) to achieve remotely-controlled brain stimulation in patients with Parkinson's Disease by applying low-energy a.c. magnetic fields to control the a.c. electric signals in the central nervous system (CNS) using intravenously injected MENs and (ii) to deliver and release on-demand (via an external field) anti-retroviral (ARV) drug AZTTP for treatment of HIV-1 reservoirs across the blood brain-barrier (BBB) $(1,97)$. In this study, we exploit this capability to achieve the field-controlled specificity of the drug-loaded MENs, as required to significantly improve the state of chemotherapy. 


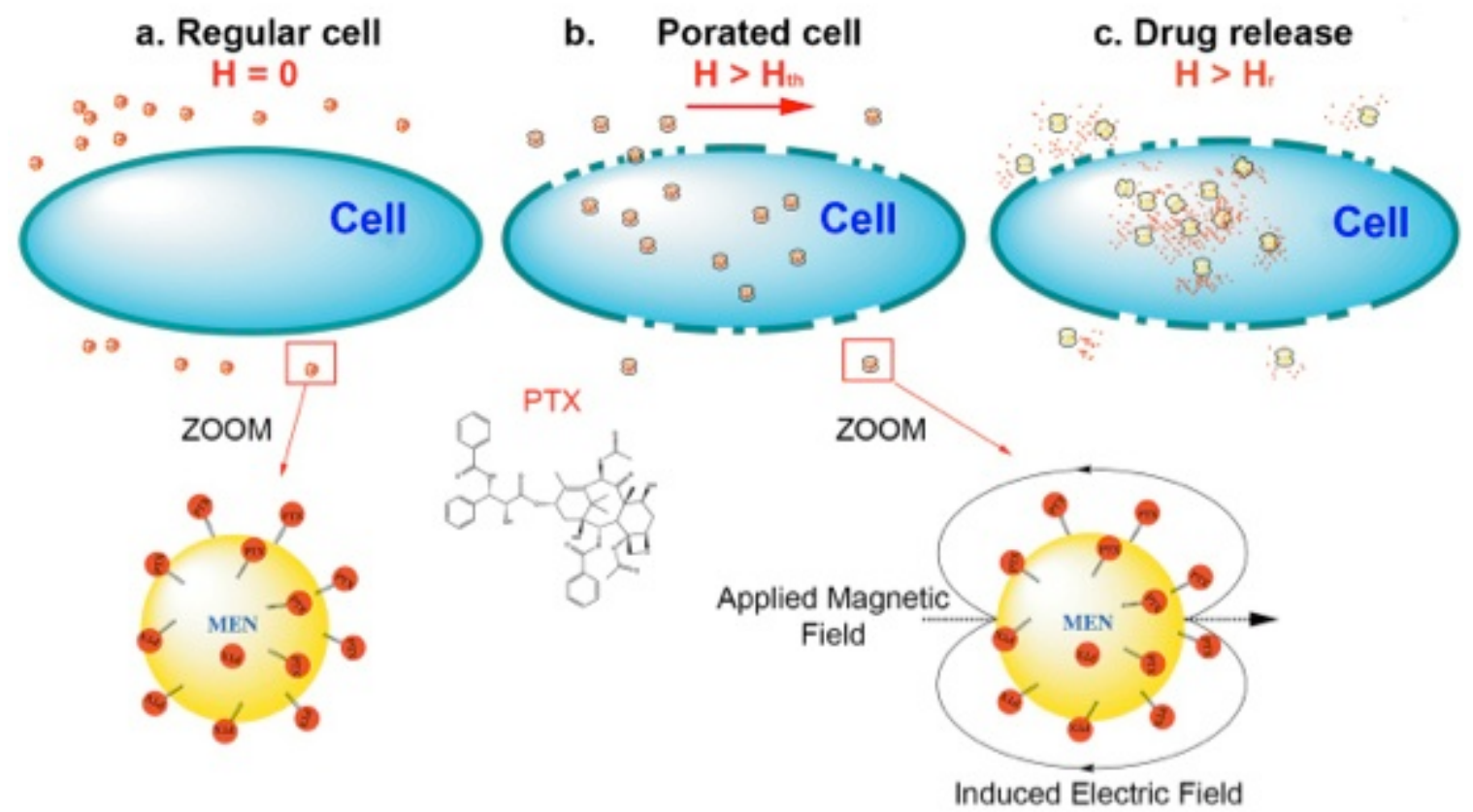

Figure 18: Hypothesis illustration: MENs as field-controlled nano-electroporation sites to let the drug through the cancer cell membranes. An artist's view that illustrates how the electric medium in the cell membrane in the vicinity of MENs can effectively act as a field-controlled array of nanoscale localized valves for the drug-loaded MENs to enter the cell. The "valves" are open as the remote magnetic field is above the first critical value, $H_{t h}$, specific to the cell. This value for the tumor cell is at least a factor of two lower than that for the healthy cell. As the field is further increased above the second critical field, $H_{r}$, the release is initiated.

With regards to achieving the required specificity to the tumor cells, the MEN's new capability to control the local electric fields remotely opens an exciting and previously unexplored path to exploit the intrinsic electric properties of the cell membrane. Due to the presence of ion channels and other electric-field driven properties, the cell membrane is an electrically polarizable medium. As a result, its properties can be significantly affected by an electric field. For example, electroporation is one such well-known characteristic that reflects the effect of the change in the membrane's porosity in the response to a large electric field (127). The electroporation has been widely studied, as a means to trigger drug delivery into the tumor cells. Through macroscale studies (with 
centimeter sizes) it is known that the electric field of higher than $1000 \mathrm{~V} / \mathrm{cm}$ creates sufficiently large pores for the drug to penetrate through the membrane. Our approach was to use MENs to generate such high large electric fields (by remotely exposing them to relatively small magnetic fields) only in a very small (nanoscale) region in the vicinity of individual MENs. By the law of scaling, when exposed to magnetic fields, MENs loaded with the drug and the biomarker-specific antibodies (for delivery to the tumor cells) can generate localized (at nanoscale) fields large enough to open up the membrane pores in their proximity only and thus let the drug inside the tumor cells. Because this process is extremely energy efficient, all the used energy goes to fulfill the main operation (of opening up the local pores, i.e. nanoscale electroporation) and consequently it doesn't result in any significant and potentially damaging energy dissipation, e.g., in terms of heat. This strongly localized near-field electric effect (with the localization defined by the size of the nanoparticles) is triggered by an external magnetic field because of the strong ME coupling within each nanoparticle. Due to the localized nanoelectroporation effect, this interaction between the MENs and the electric system of the membrane can effectively serve, as a field-controlled valve to let the drug-loaded nanoparticles enter specifically the tumor cells only. An artist's view of the main hypothesis is presented in Figure 18. In this case, the origin of the specificity to the tumor cells would be two-fold. First, the biomarker-specific antibodies steer the drug-loaded MENs (to which they also are attached) to the tumor cell membrane. Second, even higher specificity is achieved due to the fact that the tumor and healthy cells have different values of the threshold field, $\mathrm{H}_{\text {th }}$, for the valve to "open up". Indeed, it is well-known that the electric properties differ significantly between the healthy and tumor cells of the 
same type (128). For instance, the rat hepatocytes and hepatoma cells have mean membrane potentials of approximately -40 and $-20 \mathrm{mV}$, respectively. In general, the tumor cells have substantially lower values of the potential compared to that of the healthy cells. Consequently, the cancer cells must also have a significantly lower value of the threshold field to "open up" the membrane "valve" for the drug-loaded MENs to enter the cell. Considering the typical value for the ME coefficient $\alpha=100 \mathrm{~V} \mathrm{~cm}^{-1} \mathrm{Oe}^{-1}$, according to the simple isotropic expression for the ME effect, $\Delta P=\alpha H$, where $P$ and $H$ stand for the induced electric dipole field and the external magnetic field, respectively, the electric field of the order of $1000 \mathrm{~V} / \mathrm{cm}$ can be easily generated a few nanometers away from the MEN merely by applying a weak magnetic field of 10 Oe. Because this value is comparable to the field necessary for triggering the cell electroporation effect at the nanoscale, each MEN in the vicinity of the cell acts, as the cell's field-controlled nano-electroporation site. After the drug-loaded MENs penetrate into the cell cytosol through the "open" pores in the membrane, the drug can be released off the MENs by further increasing the field above the second critical value, $\mathrm{H}_{\mathrm{r}}$, necessary for overcoming the drug-MEN binding energy. We described the underlying physics of this fieldcontrolled drug release process in a recent article (1). This field strongly depends on the binding force between the MEN and the drug and consequently can be tuned in a large range through using different intermediate coating materials, field excitation frequencies and treatment durations. In summary, according to our hypothesis, there are two critical field values, $\mathrm{H}_{\text {th }}$ and $\mathrm{H}_{\mathrm{r}}$, which define the drug penetration threshold through the tumor cell membrane and the following release of the drug into the cell cytosol, respectively. To ensure adequately high efficacy of the uptake, we need $\mathrm{H}_{\mathrm{r}}>\mathrm{H}_{\text {th }}$. To ensure the 
required specificity of the uptake to the cancer cells only, the external applied field, $\mathrm{H}_{\mathrm{A}}$, needs to be higher than the release field for the tumor cells, $\mathrm{H}_{\mathrm{r}_{-} \text {cancer, }}$ and lower than the threshold field for the healthy cells, $\mathrm{H}_{\text {th }}$ healthy. In summary, using MENs not only can provide field-controlled delivery, but also can significantly improve the specificity to tumor (compared to the specificity defined by the monoclonal antibodies alone). When combined, monoclonal antibodies and MENs make a better delivery system. While the monoclonal antibodies steer the loaded drugs towards the surface of the tumor cells, the field-controlled MENs move the drugs across the cell membrane into the cytosol.

This new high-specificity nanotechnology can be applied to the treatment of cancer in general. In the current study, to prove our hypothesis, we use Epithelial Ovarian Cancer (EOC). EOC has been widely studied in the medical community (129). Cytoreductive surgery followed by chemotherapy with mitotic inhibitor Paclitaxel (PTX) with platinum is the gold standard in treating EOC. In most cases, the drug administration is intravenous (IV). Less commonly the administration is intraperitoneal (IP). As noted, there are technical considerations and limitations to IP therapy, although it is more effective than intravenous therapy. In either case, the specificity of the drug uptake is still relatively low and, as a result EOC remains a highly lethal malignancy. Therefore, the current study is relevant to this field. In addition, because of the high-specificity capability, the new nanotechnology can be used for targeted treatment of both localized and metastasized tumor cells. 


\subsection{Experimental methods}

\subsubsection{Preparation of $\mathrm{CoFe}_{2} \mathrm{O}_{4}-\mathrm{BaTiO}_{3}$ Coreshell MENs}

For preparation of coreshell MENs please refere to section 2.2.1.

\subsubsection{Preparation of $G M O-M E N S$}

In-order to load the PTX drug onto the MENs' surface; the nanoparticles were first coated with GMO to adjust the release field at about $30 \mathrm{Oe}$, as required for this application. To achieve this, $1 \mathrm{mg}$ of GMO was added to $5 \mathrm{mg}$ of MENs in $5 \mathrm{ml}$ of the PBS buffer. The mixture was then incubated for 12 hours while being slowly rotated in order to achieve uniform coating. Upon completion of the incubation process, the nanoparticles were centrifuged at $20,000 \mathrm{rpm}$ for 20 minutes at $10^{\circ} \mathrm{C}$. The pellet was washed in ethyl acetate:acetone (70:30) solution and re-centrifuged. The washing process was repeated thrice to completely remove the excess unbound GMO. Finally, the obtained pellet was lyophilized for 48 hours and stored for further use. The results of the energy dispersion spectrometry (EDS) that depict the materials composition of GMOMENs are summarized in Figure 19. 
Full scale counts: 1806

Base(3)_pt1

Cursor: $\quad 0.793 \mathrm{keV}$

89 Counts

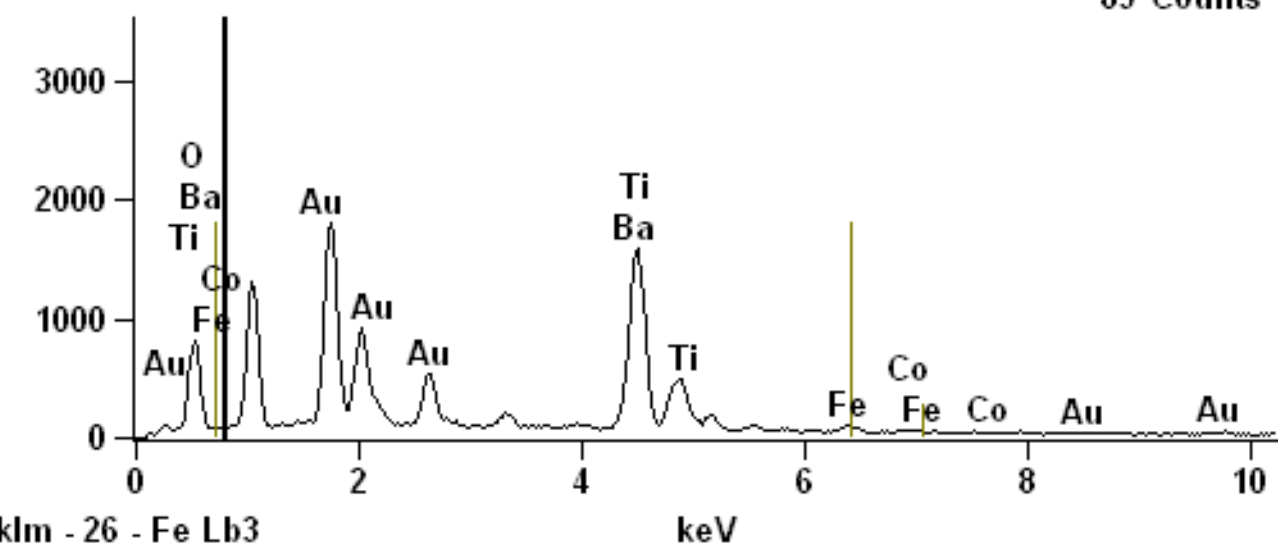

klm - 26 - Fe Lb3

keV

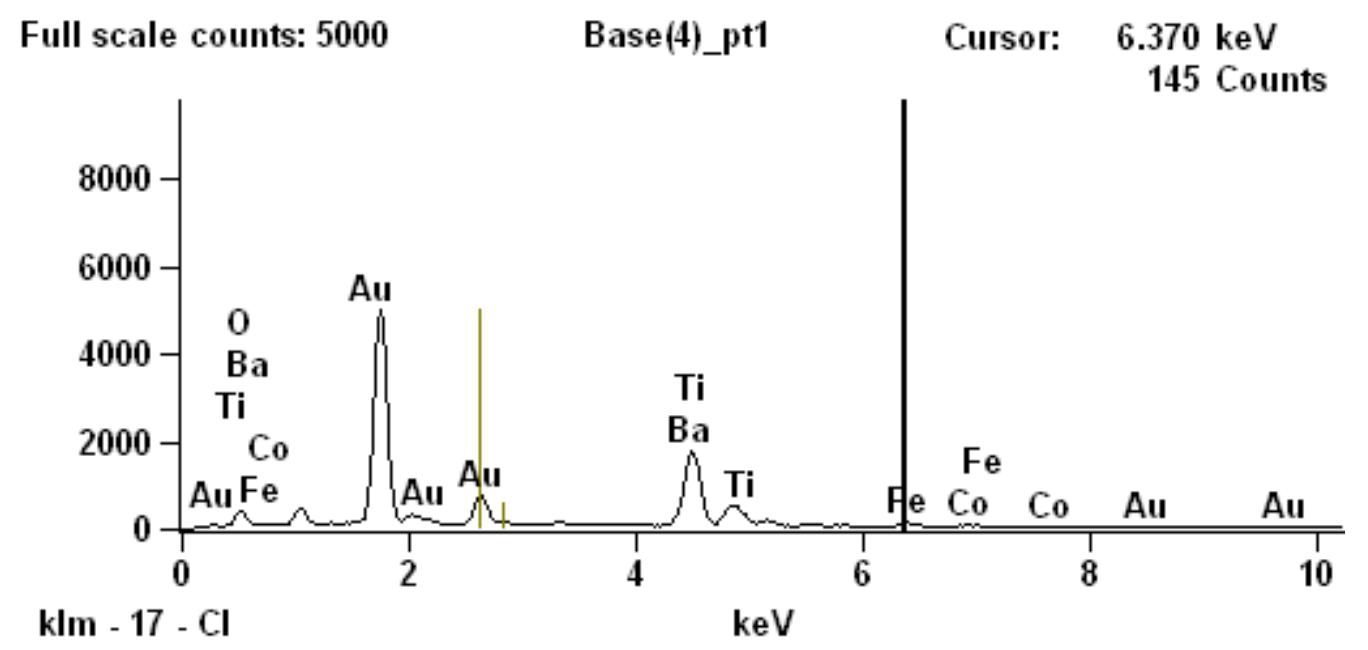

Figure 19: Energy dispersion spectroscopy of MENs. Energy dispersion spectroscopy (EDS) results depict the composition of (a) MENs only and (b) GMO-coated MENs.

\subsubsection{Preparation of HER-2 biomarker antibody conjugated GMO-MENS}

HER-2 biomarker antibodies were covalently attached onto the GMO-MENs' surface according to the protocol described by Kocbek et al (130). In-order to covalently attach the HER-2 antibodies, the nanoparticle surface was preliminarily functionalized. For this, 1mg of GMO-MENs were added to $500 \mu$ l of the PBS buffer ( $\mathrm{pH}$ 7.4). To this solution, 
$25 \mu 1$ of $\mathrm{N}$-(3-Dimethylaminopropyl)-N'-ethyl-carbodiimide hydrochloride (EDC) and $25 \mu l$ of $\mathrm{N}$-hydroxysuccinimide (NHS), at $1 \mathrm{mg} / \mathrm{ml}$ concentration in the PBS buffer ( $\mathrm{pH}$ 7.4) were added. The solution was incubated for 4 hours at room temperature while being stirred slowly. Then, the sample was centrifuged at $14,000 \mathrm{rpm}$ for 10 minutes at $10^{\circ} \mathrm{C}$ and the pellet was washed three times with $1 \mathrm{ml}$ of the PBS buffer ( $\mathrm{pH}$ 7.4). To bind HER-2 antibodies to the functionalized MENs, $10 \mu \mathrm{l}$ of the antibodies $(1 \mathrm{mg} / \mathrm{ml})$ was added to the pellet along with $300 \mu 1$ of the PBS buffer ( $\mathrm{pH}$ 7.4). The solution was incubated for 2 hours while being rotated slowly and kept further at $4^{\circ} \mathrm{C}$ overnight. The solution was centrifuged at $14,000 \mathrm{rpm}$ for 10 minutes at $10^{\circ} \mathrm{C}$. The pellet was washed thrice with $1 \mathrm{ml}$ of the PBS buffer ( $\mathrm{pH}$ 7.4) to remove any excess antibody. The supernatant was collected to determine the amount of the unconjugated HER-2 protein by comparing to the standard plot. A standard calibration plot for HER-2 was obtained by varying the concentration in the range of $1.25-10 \mu \mathrm{g} / \mathrm{ml}$ using Bio-Rad protein assay kit (Braford method) through measuring the absorbance at $595 \mathrm{~nm}$ using spectrophotometer Cary-100 (Figure 20). The percentage of the conjugated HER-2 was obtained using the following expression: the percentage of HER-2 conjugated $=($ the total amount of HER-2 added - the amount of the unconjugated HER2 present in the supernatant) $\times 100$. The results indicated that over $70 \%$ of the HER-2 antibodies were conjugated to the GMOMENs' surface. 


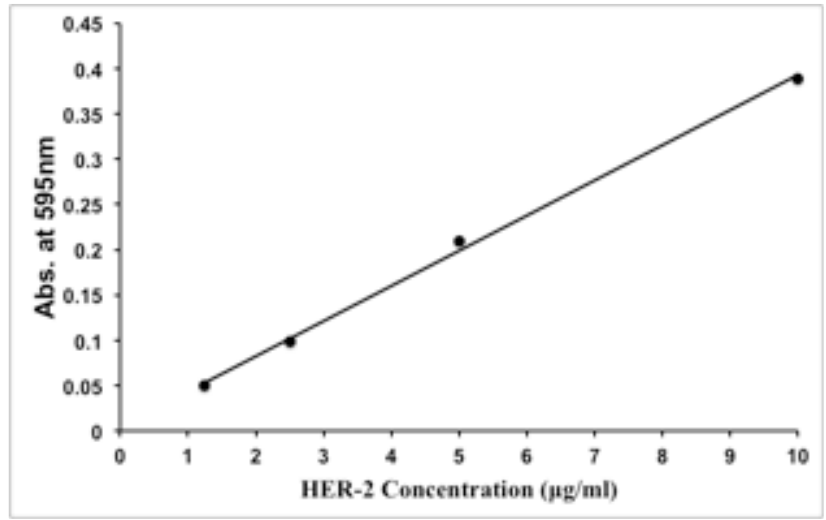

Figure 20: Standard HER-2 antibody concentration calibration curve. Standard HER-2 Antibody plot was obtained using Bio-Rad protein assay kit at $595 \mathrm{~nm}$ absorbance.

\subsubsection{Preparation of drug loaded MENs}

After $50 \mu \mathrm{g}$ of PTX drug was added to the solution of $900 \mu$ l of the modified PBS (MPBS) buffer and $100 \mu \mathrm{l}$ of the desired MEN combination (i.e. MENs, GMO-MENs, and HER-2-GMO-MENs at a $5 \mathrm{mg} / \mathrm{ml}$ concentration), the solution was incubated for 3 hours while stirred slowly to obtain uniform binding. Then, the solution was centrifuged at $14,000 \mathrm{rpm}$ for 10 minutes at $10^{\circ} \mathrm{C}$ to remove any unbounded drug. The supernatant was isolated and absorbance was measured spectrophometrically at $230 \mathrm{~nm}$ using Cary100 UV-VIS spectrophotometer (131). The PTX loading percentage is shown in Figure 21. A standard calibration plot for PTX was obtained by varying the drug concentration from 5 to $80 \mu \mathrm{g}$ in $1 \mathrm{ml}$ of the MPBS solution and the absorbance was measured at 230 $\mathrm{nm}$. The absorption maxima and the standard linear calibration plot of PTX at different drug concentration values are shown in Figure 22. 


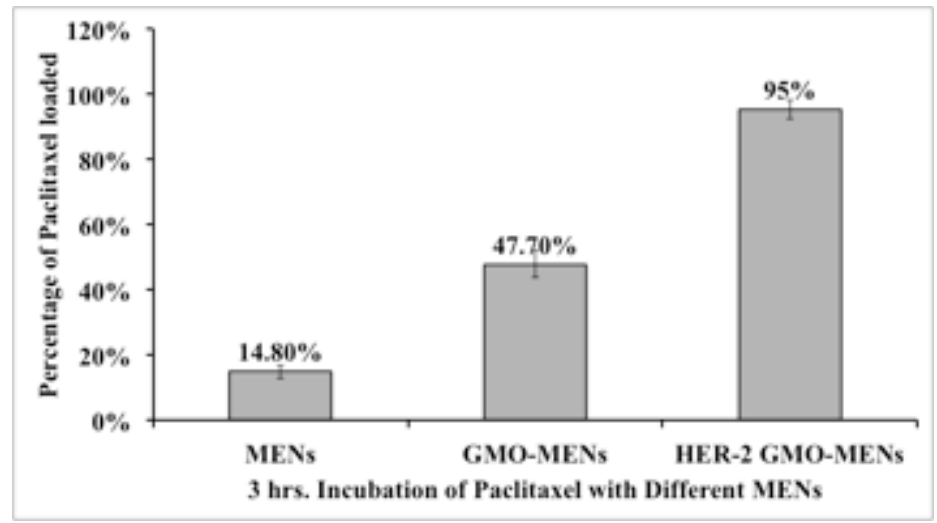

Figure 21: PTX drug loading percentages. PTX drug loading percentage for MENs, GMO-MENs and HER-2-GMO-MENs after 3 hrs. incubation (n=3).
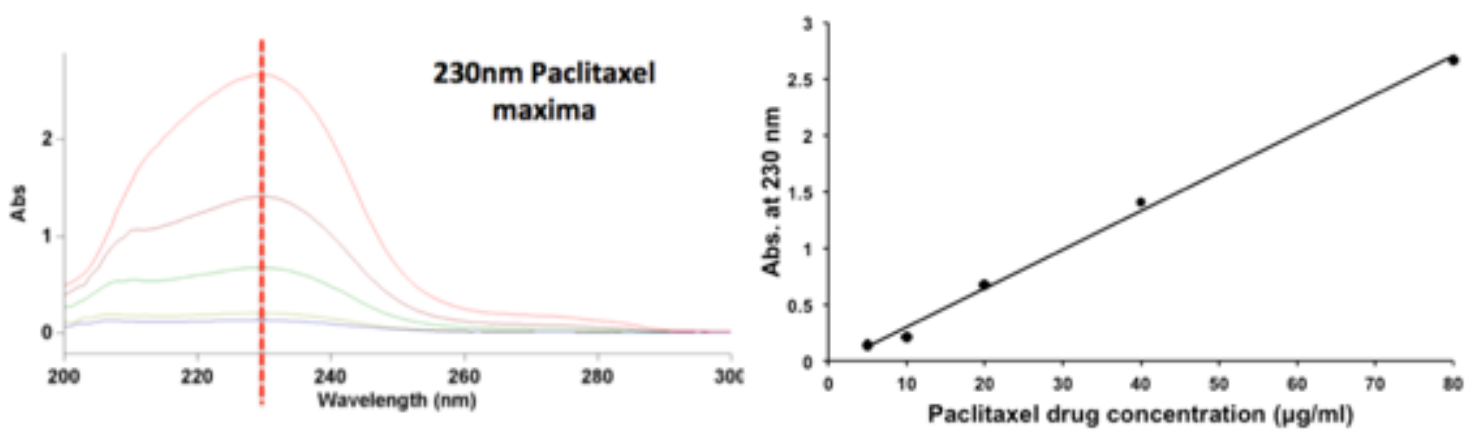

Figure 22: PTX calibration plot. The absorption maxima and the standard linear calibration plot for PTX at different drug concentration values determined spectrophotometrically.

\subsubsection{Cell culture experiments}

Cell culture experiments were performed using human ovarian carcinoma cell line (SKOV-3) purchased from American Type Culture Collection (Manassas, VA) and were cultured in McCoy's 5A medium (Life Technologies, NY) supplemented with $10 \%$ fetal bovine serum (Sigma-Alrich) and 1\% penicillin-streptomycin (science-cell). Human Ovarian Microvasicular endothelial cells (HOMEC) from ScienceCell (Carisbad, CA) and were cultured in endothelial cell medium with endothelial cell growth supplement 
(1\%), fetal bovine serum (5\%), and penicillin-streptomycin (1\%). All the cells were cultured at $37^{\circ} \mathrm{C}$ cell incubator with a $5 \% \mathrm{CO}_{2}$ and humidified atmosphere.

\subsubsection{Fluorescence measurements and confocal imaging of drug uptake by SKOV-3 cells for different drug-carrier combinations}

Cellular drug uptake measurements and fluorescence imaging were performed using an Oregon Green ${ }^{\circledR} 488$ paclitaxel (also called Flutax-2). The experiments were performed in dark. For the fluorescence measurements, the SKOV-3 cells were cultured in 24-well plate at a density of $2 \times 10^{5}$ cell per well. After 24 -hour incubation at $37^{\circ} \mathrm{C}$, the cell medium was replaced with $1 \mathrm{ml} /$ well of the medium containing either one of the four desired drug forms. The concentration of Flutax- 2 was normalized to $0.75 \mu \mathrm{M}(1.76$ $\mu \mathrm{g} / \mathrm{ml})$ for all the combinations. The cell culture plate was returned to the incubator and incubated for 10 hours. In addition, a set of controls containing no drug for all the combinations was cultured under similar conditions. The cell culture plates containing the Flutax-2-MNs and Flutax-2-GMO-MENs were kept under a 30-Oe field. Upon completion of the 10-hour incubation process, the cells were removed from incubator and the cell culture medium was discarded. The cells were washed with ice-cold PBS buffer thrice. Then, $1 \mathrm{ml}$ of dimethyl sufloxide (DMSO) was added to each well and incubated for 2 hours at $37^{\circ} \mathrm{C}$. After two hours, a rubber policeman was used to ensure the complete removal of the attached cells. The solution was centrifuged at 14,000 rpm for 10 minutes at $4^{\circ} \mathrm{C}$ to obtain the cell lysate. The cell lysate along with the in-taken Flutax-

2-GMO-MENs was collected and measured for the fluorescence of Flutax-2 (using 
BioTek instruments, synergy HT) at $\lambda_{\mathrm{ex}}=496 \mathrm{~nm}$ and $\lambda_{\mathrm{em}}=524 \mathrm{~nm}$ to determine the concentration. All the fluorescence measurements were recorded by subtracting the corresponding controls to adjust the background fluorescence from the cellular components. The protein content of the cell lysate was determined using Bio-Rad protein assay kit (Braford method) by measuring the absorbance at $595 \mathrm{~nm}$ using a Cary $100 \mathrm{UV}$ VIS spectrophotometer. As for the imaging studies, the cells were cultured on glass cover slips $\left(1 \times 1 \mathrm{in}^{2}\right)$ pre-coated with the poly-L-Lysine (used as a cell adhesion promoter) in a 6-well cell culture plate at a density of $5 \times 10^{4}$ were cultured and let rested for about 10 minutes. Then, $2 \mathrm{ml}$ of Cell medium was added along the walls of the wells. The cell culture plate was incubated for 24 hours at $37^{\circ} \mathrm{C}$. The cells were supplied with the fresh cell medium that contained either of the four drug forms under study normalized to the $0.75 \mu \mathrm{M}(1.76 \mu \mathrm{g} / \mathrm{ml})$ of Flutax-2 concentration. The cell plate containing the Flutax-2GMO-MENs was kept under a 30-Oe magnetic field. The cell plates were incubated for 10 hours at $37^{\circ} \mathrm{C}$. After the incubation process, cover slips were washed three times with the PBS buffer and fixed with $4 \%$ paraformaldehyde for 30 minutes followed by washing thrice with the PBS buffer. The cover slips were mounted onto a glass slide using a mounting medium (ProLong Gold Antifade Reagent). The excess mounting medium was removed by placing a small piece of Whatman paper around the edges. After the samples were dried for 2 hours, they were imaged through confocal microscopy (TCS SP2, Leica Microsystems, Germany) at $488 \mathrm{~nm}(100 \%)$ illusion of an argon-ion laser using $60 \mathrm{X}$ oil immersion objectives with a high numeric aperture and $1 \mathrm{X}$ confocal electronic zoom settings to visualize cells. 


\subsection{Results}

Here, we conduct in-vitro studies on human ovarian carcinoma cell (SKOV-3) and healthy ovarian cell (HOMEC) lines. We demonstrate that high-specificity uptake of PTX-loaded 30-nm $\mathrm{CoFe}_{2} \mathrm{O}_{4} @ \mathrm{BaTiO}_{3}$ MENs could be triggered by a low-energy 30-Oe d.c. external magnetic field with negligible heat dissipation. Through kinetics studies we confirm that the drug penetrates through the tumor cell membrane and completely eradicates the cells within a 24-hour period without affecting the surrounding healthy cells. The procedures to fabricate the nanoparticles with different sets of coatings and drug loadings are described in Section 4.2.2. The release threshold field, $\mathrm{H}_{\mathrm{r}}$, could be controlled in a wide range, from 10 Oe to substantially over 200 Oe, through different intermediate layers/coatings. A comparative analysis of the effect of the intermediate layer type on $\mathrm{H}_{\mathrm{r}}$ is summarized in Figure 36. By default, in order to provide adequate coupling between the MENs and Flutax-2 (to provide the initial release field of the order of $30 \mathrm{Oe}$ ), before being loaded with the drug, the MENs were coated with 3-Angstrom thick glycerol monooleate (GMO) layers. The zeta-potential and size of the MENs, GMO-MENs, HER2-GMO-MENs, and PTX-GMO-MENs are shown in Table 5. For the purpose of a comparative analysis, we studied the following combinations of nanoparticles: (i) MENs loaded with PTX, (2) MENs loaded with PTX and the popular cancer biomarker HER-2 antibody, (3) free PTX, and (4) conventional MNs loaded with PTX. As the conventional MNs, 30-nm magnetite nanoparticles were used. 
Table 5: The Malvern Zeta-sizer was used to measures size and Zeta-potential of the MENs, GMO-MENs, HER2-GMO-MENs, and PTX-GMO-MENs $(n=3)$. Concentration of the nanoparticles used for the measurements was $500 \mathrm{ug} / \mathrm{ml}$ of D.I water. Note: The measurements represented are the average of three independent measurements.

\begin{tabular}{|c|c|c|c|c|}
\hline \multirow{2}{*}{$\begin{array}{c}\text { Type of } \\
\text { measurement }\end{array}$} & MENs & GMO-MENs & $\begin{array}{c}\text { HER2-GMO- } \\
\text { MENs }\end{array}$ & $\begin{array}{c}\text { PTX-GMO- } \\
\text { MENs }\end{array}$ \\
\cline { 2 - 5 } & $28.6 \pm 7.5$ & $30.9 \pm 8.6$ & $43.0 \pm 3.6$ & $44 \pm 6.6$ \\
\hline Size (nm) & $-45.0 \pm 1.7$ & $-41.6 \pm 0.3$ & $-26.3 \pm 0.4$ & $-40.7 \pm 0.1$ \\
\hline $\begin{array}{c}\text { Zeta-Potential } \\
(\mathrm{mV})\end{array}$ & -40.6 & \\
\hline
\end{tabular}

\subsubsection{Field-controlled drug release by MEN-based carriers}

Drug release from these different MEN-based combinations was triggered by a magnetic field at different strengths and frequencies, according to the physics described in our earlier paper on the release of ARV drug AZTTP for treatment of HIV-1 virus in the brain (1). The pellet obtained after the drug loading procedure was washed thrice with the phosphate-buffered saline (PBS) buffer, to remove any residual unbounded drug. The drug-loaded-MENs' pellet was added to $1 \mathrm{ml}$ of the PBS buffer in a vial and subjected to a d.c. or a.c. magnetic field using a pair of Helmholtz coils connected to a d.c. or a.c. power supply, respectively. After exposing the vial to any magnetic field environment under study, the supernatant was obtained by spinning the sample at 3,000 rpm for 5 minutes and at $10^{\circ} \mathrm{C}$. The supernatant was measured for the amount of the released drug spectrophotometerically through the absorbance at the PTX maximum wavelength of 230 nm (131). 


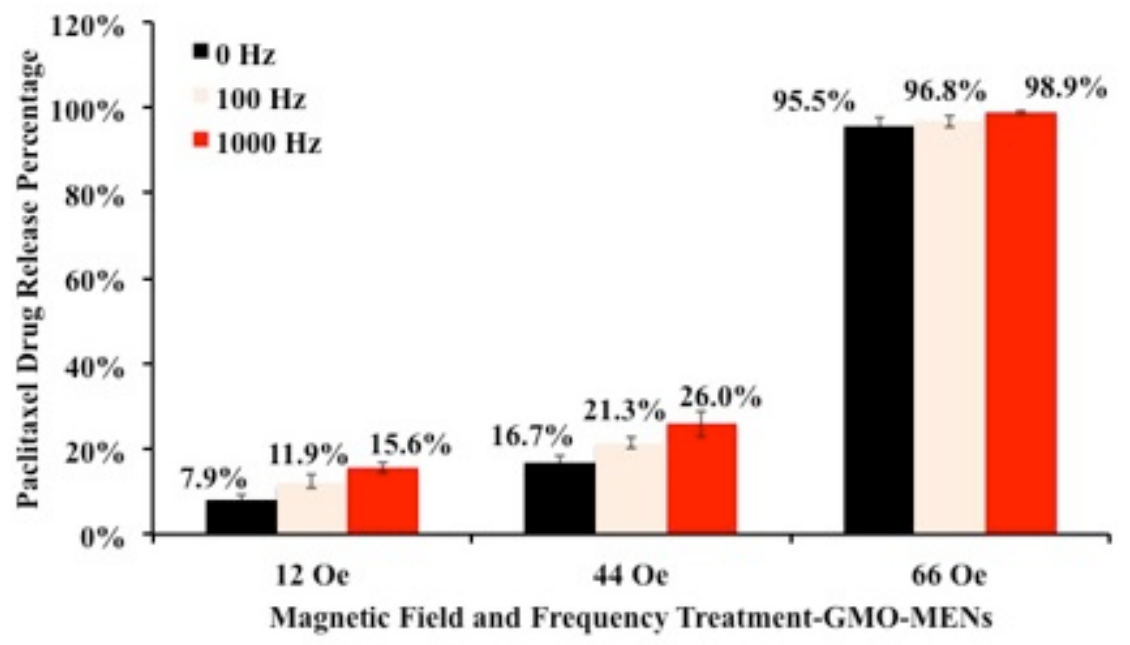

A.

B.

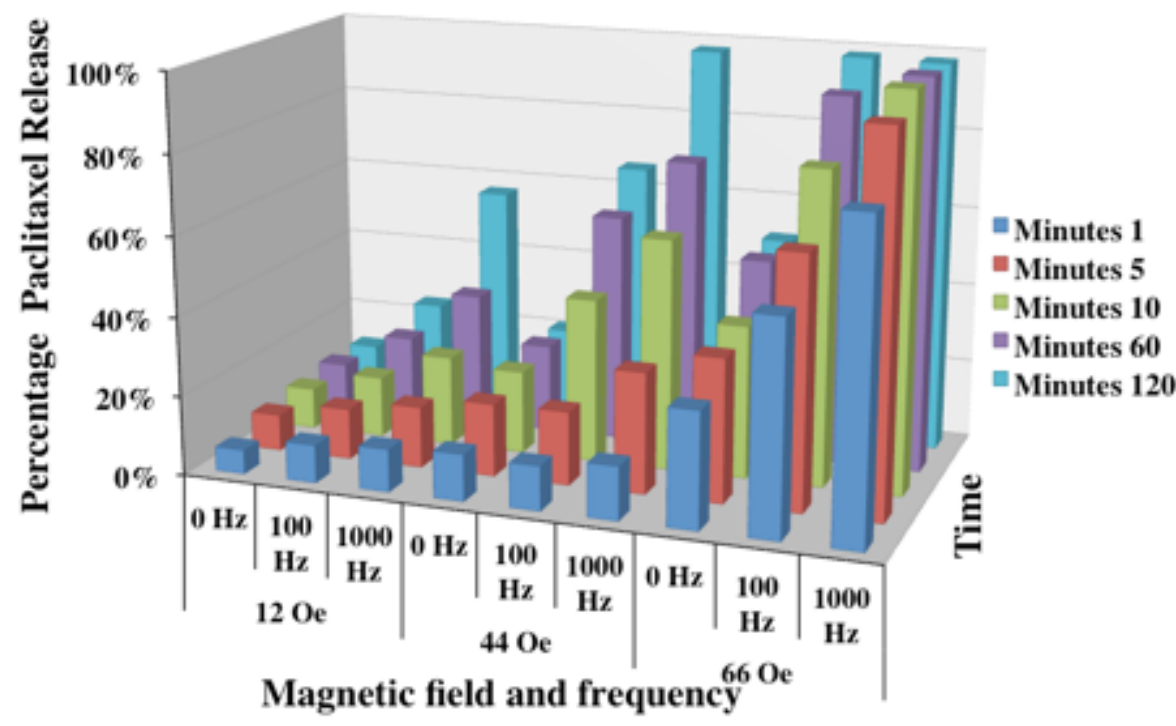

Figure 23: Photo-absorption measurements of the release kinetics. (A) PTX drug release form GMO-MENs, when treated at varying magnetic field strengths and frequencies after 1-minute treatment (n=3). (B) 3-D Chart that illustrates the kinetics of the drug release. The data were measured spectrophotometrically as the absorbance at $230 \mathrm{~nm}$ wavelength. 
Table 6: The field release dependence on the field, frequency, and the treatment duration. The table shows the percentage of the field-triggered released PTX at different combinations of the magnetic field strength $(12,44$, and $66 \mathrm{Oe})$ and frequency $(0,100$, and $1000 \mathrm{~Hz})$ and the treatment duration $(1,5,10,60$, and 120 minutes).

\begin{tabular}{|c|c|c|c|c|c|c|}
\hline \multirow{2}{*}{$\begin{array}{c}\text { Field } \\
\text { Strength }\end{array}$} & \multirow{2}{*}{$\begin{array}{c}\text { Frequenc } \\
\mathrm{y}\end{array}$} & \multicolumn{5}{|c|}{$\begin{array}{c}\text { Percentage Paclitaxel Release at Different } \\
\text { Treatment Time Durations (Minutes) }\end{array}$} \\
\cline { 2 - 7 } & & 1 & 5 & 10 & 60 & 120 \\
\hline \multirow{3}{*}{$12 \mathrm{Oe}$} & $0 \mathrm{~Hz}$ & $6.1 \%$ & $9.1 \%$ & $10.3 \%$ & $11.4 \%$ & $11.1 \%$ \\
\cline { 2 - 7 } & $100 \mathrm{~Hz}$ & $9.5 \%$ & $12.8 \%$ & $15.3 \%$ & $20.5 \%$ & $24.4 \%$ \\
\cline { 2 - 7 } & $1000 \mathrm{~Hz}$ & $10.7 \%$ & $15.4 \%$ & $22.6 \%$ & $33.7 \%$ & $56.7 \%$ \\
\hline \multirow{3}{*}{$44 \mathrm{Oe}$} & $0 \mathrm{~Hz}$ & $11.8 \%$ & $18.3 \%$ & $20.9 \%$ & $22.2 \%$ & $21.1 \%$ \\
\cline { 2 - 7 } & $100 \mathrm{~Hz}$ & $11.2 \%$ & $18.5 \%$ & $41.3 \%$ & $57.9 \%$ & $66.2 \%$ \\
\cline { 2 - 7 } & $1000 \mathrm{~Hz}$ & $13.4 \%$ & $30.4 \%$ & $58.2 \%$ & $73.2 \%$ & $97.8 \%$ \\
\hline \multirow{3}{*}{$66 \mathrm{Oe}$} & $0 \mathrm{~Hz}$ & $29.1 \%$ & $36 \%$ & $38.4 \%$ & $49.8 \%$ & $50.5 \%$ \\
\cline { 2 - 7 } & $100 \mathrm{~Hz}$ & $53.1 \%$ & $62.9 \%$ & $78.5 \%$ & $92.3 \%$ & $98.5 \%$ \\
\cline { 2 - 7 } & $1000 \mathrm{~Hz}$ & $78.1 \%$ & $93.7 \%$ & $98.5 \%$ & $98.2 \%$ & $98.2 \%$ \\
\hline
\end{tabular}

The results of the field-controlled drug release spectrophotometry (absorption) experiments are summarized in Figure 23. Figure 23A shows the percentage of the drug release after a 1-minute exposure to a magnetic field at three strengths, 12, 44, and $66 \mathrm{Oe}$, respectively, for three different frequencies, 0, 100, and $1000 \mathrm{~Hz}$, respectively. As expected and previously explained, for each frequency, there was a critical field, $\mathrm{H}_{\mathrm{r}}$, at which the drug release was significantly boosted. The increase of the frequency in the range up to $1000 \mathrm{~Hz}$ under study increased the release efficacy (by over 40\%) especially at the low field range. Figure 23B illustrates the kinetics of the field-strength-frequency dependence of the release for the five values of the field exposure times, 1, 5, 10, 60, and 120 minutes, respectively. The quantitative values are also presented in Table S1. For every exposure time setting, a fresh solution with PTX-loaded GMO-coated MENs was used. The field-triggered drug release was also confirmed through atomic force microscopy (AFM), Fourier Transform Infra-Red (FTIR), mass spectrometry, and X-ray 
diffraction (XRD) pattern studies. The results are summarized in Figure 24, Figure 25,

Figure 26, and Figure 27 respectively
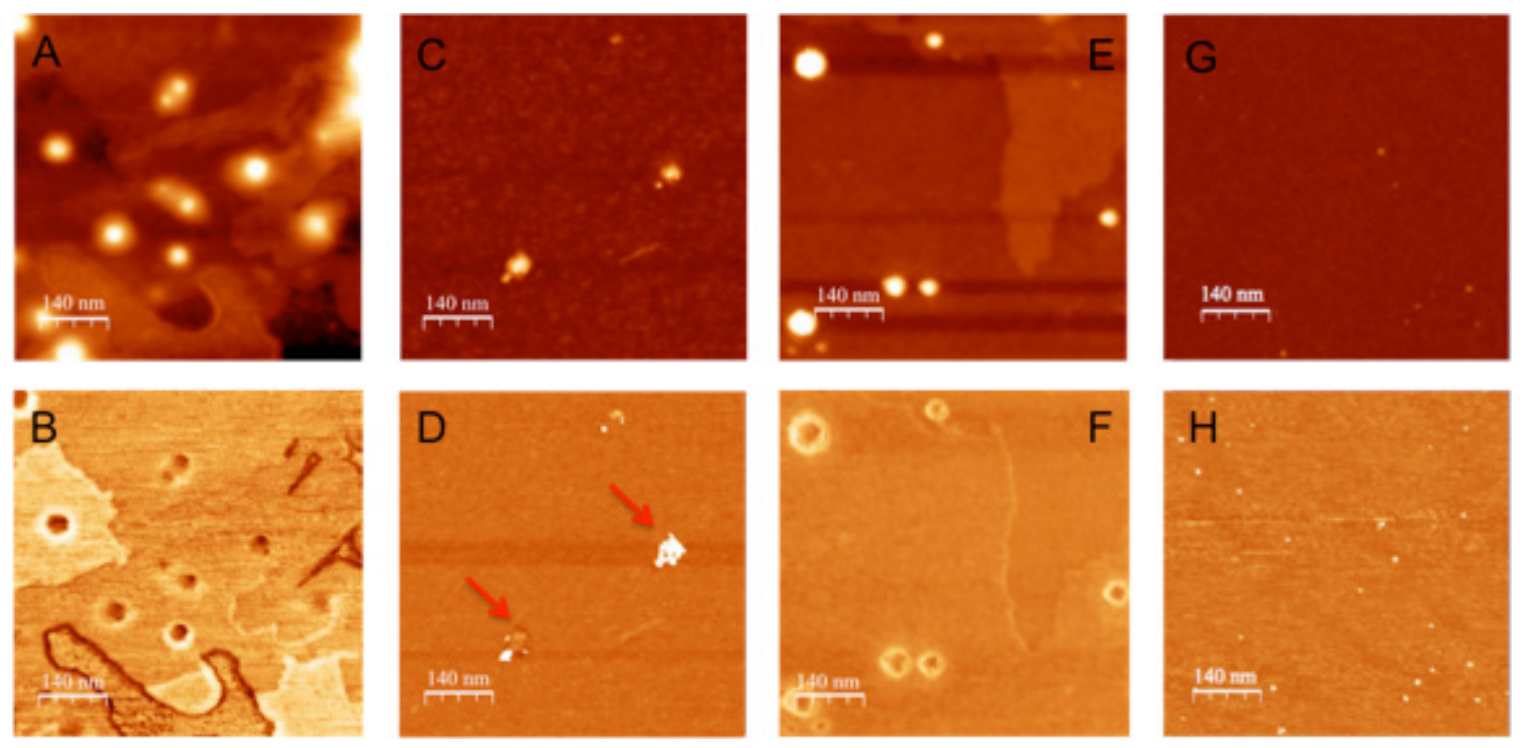

Figure 24: Atomic force microscopy. Topography and phase-contrast atomic force microscopy (AFM) images of (A and B) GMO-MENs only, (C and D) GMO-MENs loaded with PTX, (E and F) GMO-MENs after the 36-Oe 1000-Hz field-triggered release, and (G and H) PTX only. The imaging was performed in Tapping Mode ${ }^{\mathrm{TM}}$ using $^{-}$ Nanoscope IIIa Multimode AFM (Veeco Metrology, CA). The samples were specially prepared for the imaging by dispersing desired MENs on the silicon wafer surface $\left(1 \times 1 \mathrm{~cm}^{2}\right)$. To achieve uniform dispersion of MENs, the nanoparticles of choice were first mixed in chloroform solution ( $1 \mathrm{mg}$ of particles in $600 \mu \mathrm{l}$ of chloroform). Then, $100 \mu \mathrm{l}$ of this solution was mixed with $200 \mu \mathrm{l}$ of isopropanol and the solution was set to 3500-rpm centrifugation for 3 minutes. Finally, the obtained pellet of MENs was dissolved in $200 \mu$ l of Chloroform and 20 $\mu \mathrm{l}$ of this solution was placed on a silicon wafer and then dried at room temperature overnight. The silicon wafer containing the desired MENs were glued on to a metallic sample puck and mounted on the AFM stage. All the images were performed in $256 \times 256$ scan resolution at a scan rate of $0.5 \mathrm{~Hz}$ at a $40-\mathrm{nm} \mathrm{Z}$-scale and a $60^{\circ}$-Phase angle. 


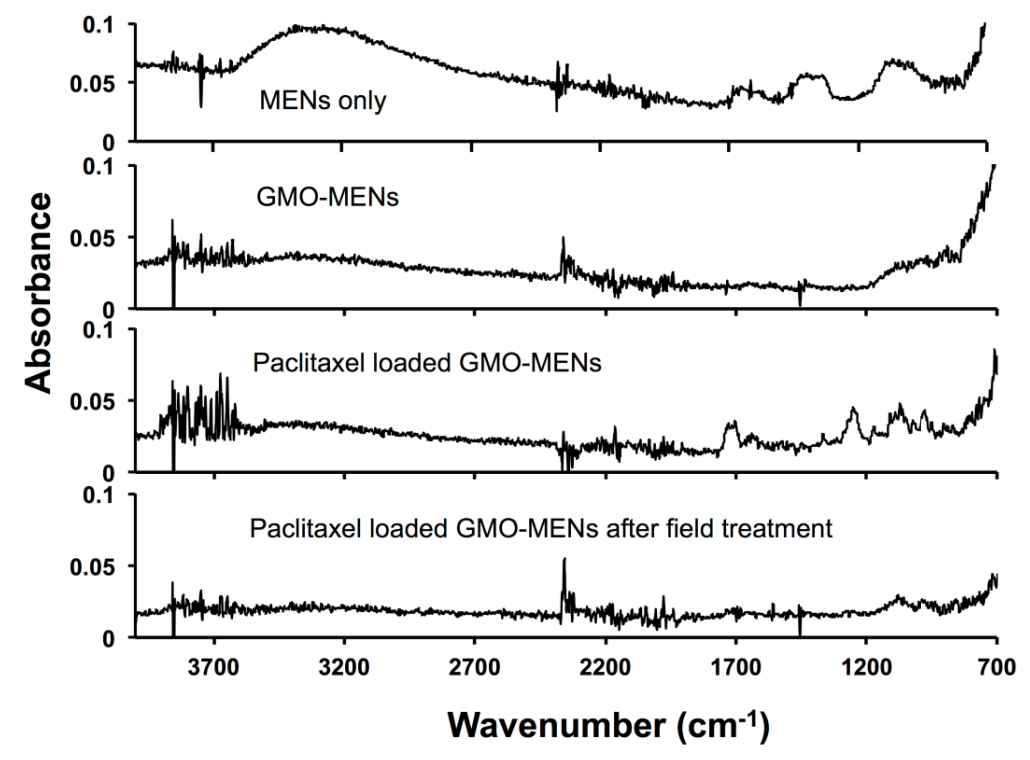

Figure 25: Four transform infra-red studies. Fourier Transform Infra-Red (FTIR) results showing the spectrum change at different stages of the drug release.

A.

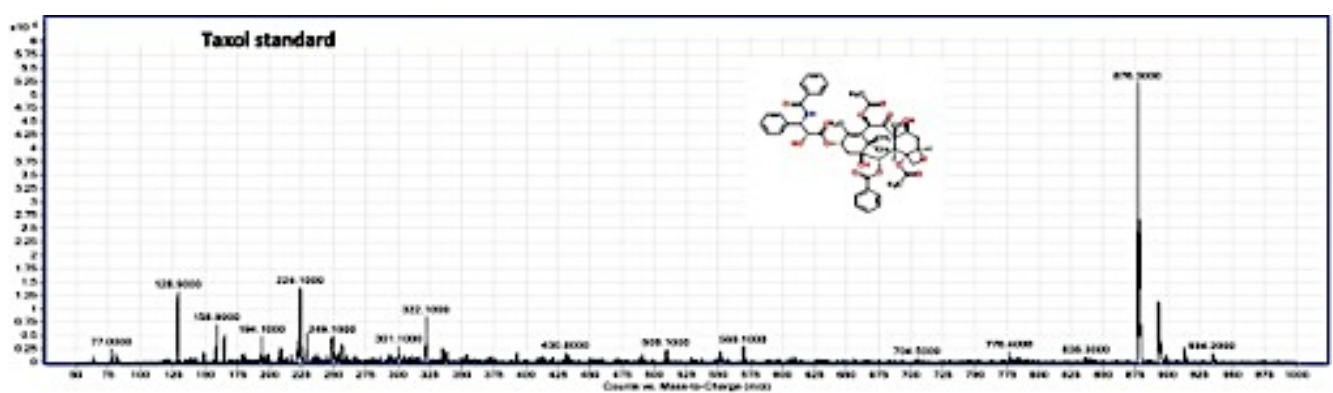

B.
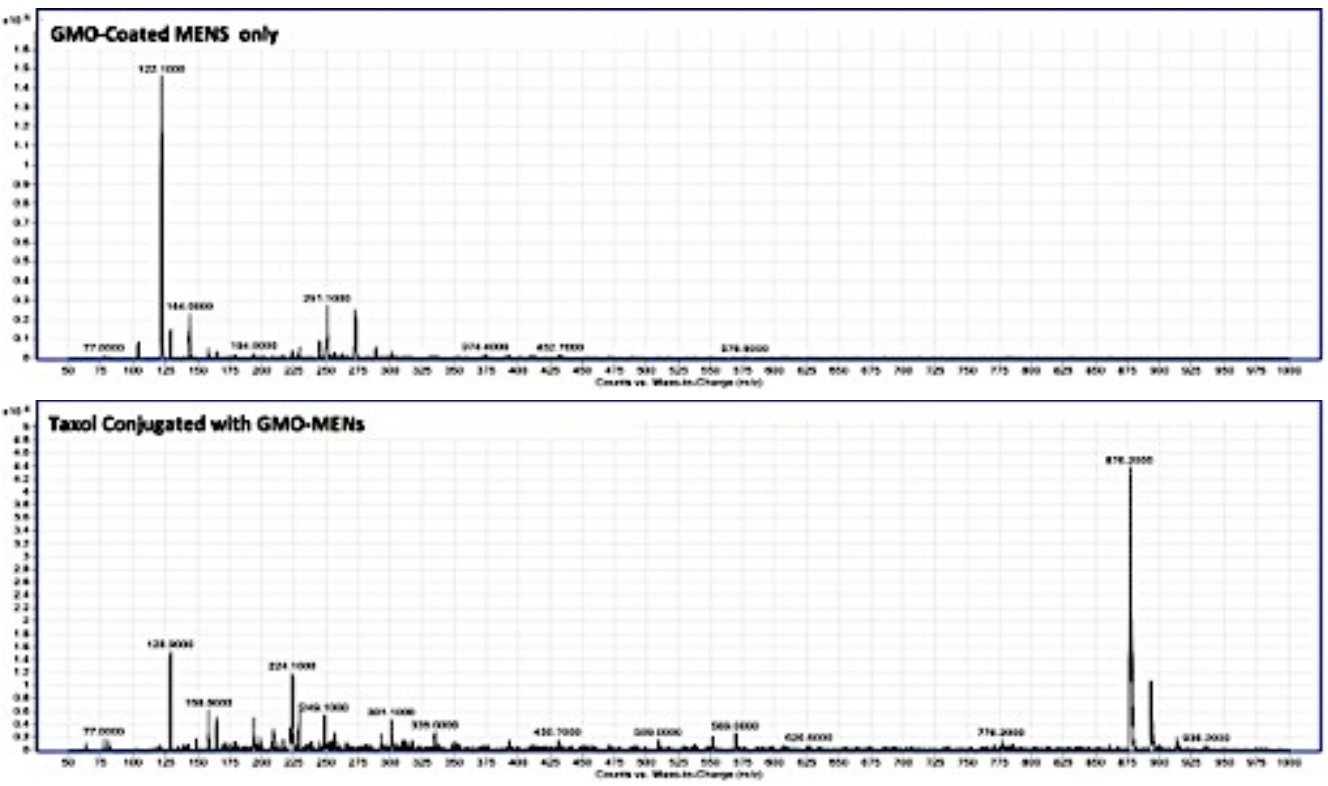


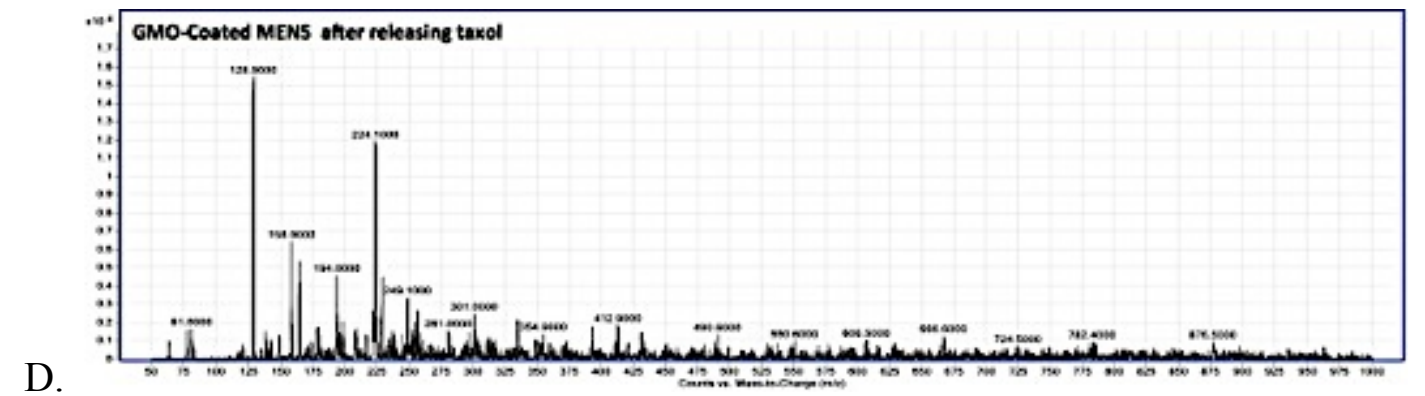

D.

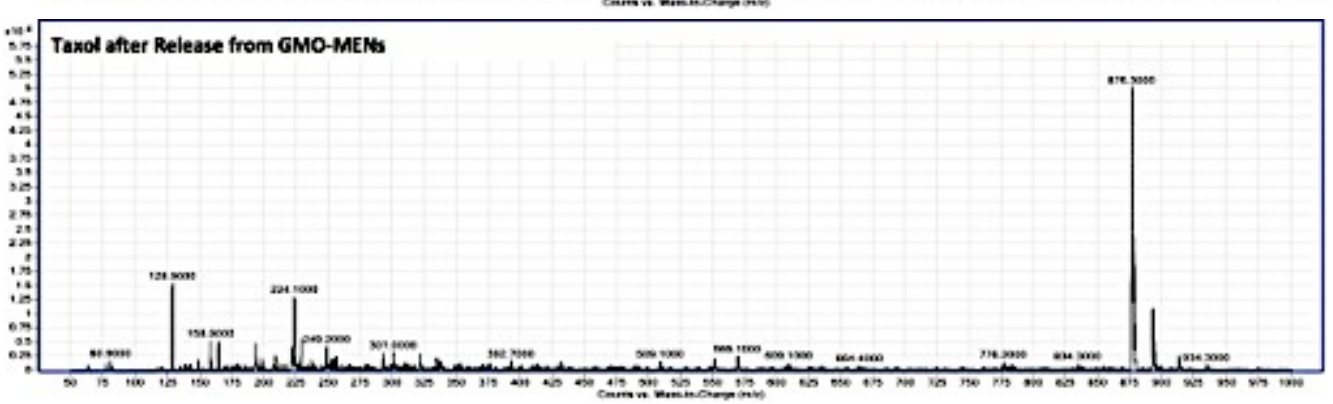

E.

Figure 26: Mass spectroscopy results showing (A) the significant peak of PTX at $876 \mathrm{~nm}$ in the MBPS buffer, (B) GMO-MENs in the MPBS buffer, (C) the significant peak of PTX at $876 \mathrm{~nm}$ when conjugated to the GMOMENs in the MPBS buffer, (D) PTX-GMO-MENs after the field treatment, (E) PTX after the release.

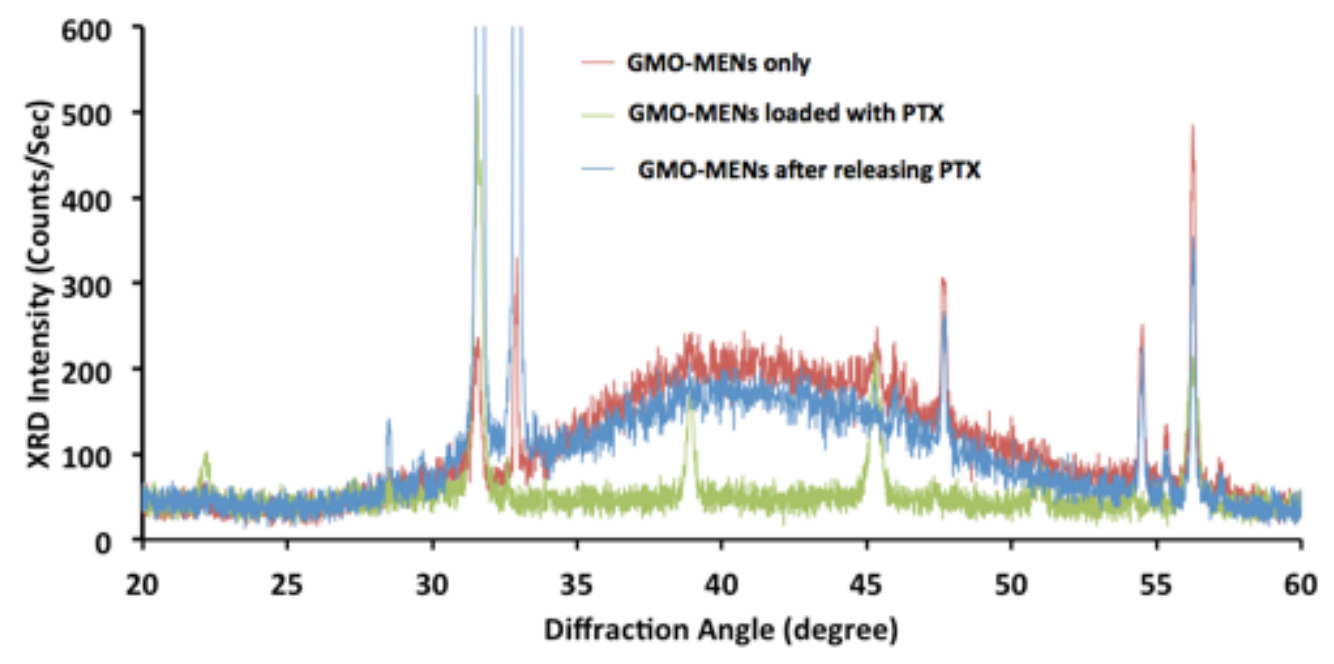

Figure 27: XRD pattern. XRD results of GMO-MENs at the three important phases of the release: (RED) before and (GREEN) after binding with PTX before the release and (BLUE) after the field-triggered release. Prior to performing the measurements, the nanoparticles were dispersed on a Silicon wafer and dried for 24 hours. 


\subsubsection{Field-controlled drug uptake by tumor cells through the MEN-induced nano-electroporation}

Fluorescent cellular drug uptake experiments were performed on the SKOV-3 cells using the four different drug forms under study, (i) free Flutax-2, (ii) Flutax-2 bound to the conventional MNs, (iii) Flutax-2 bound to GMO-MENs, and (iv) Flutax-2 bound to HER2-GMO-MENs, respectively. The obtained Flutax-2 concentration was normalized to the protein amount. The results of the experiment performed in triplicates are shown in Figure 28. These results showed that the drug uptake increased by a factor of five for the drug carried by field-controlled MENs compared to the drug driven by the HER-2 antibodies.

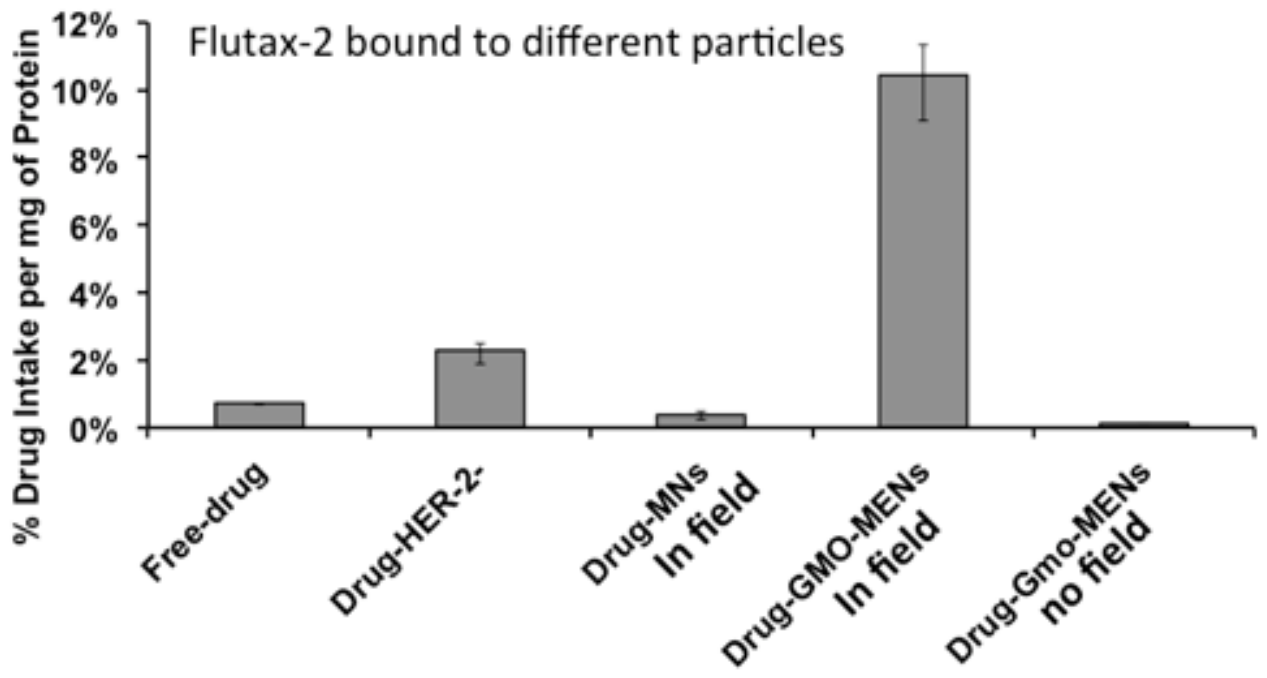

Figure 28: Drug uptake by cancer cells via different carriers. Comparison of four different forms of Flutax-2 drug intake by SKOV-3 cells $(n=3)$. 


\subsubsection{Confocal microscopy to visualize the internal drug localization in SKOV-3}

cell lines

To visualize the internal localization of each of the four drug forms under study, (i) free Flutax-2, (ii) Flutax-2-GMO-MENs, (iii) Flutax-2-HER-2, and (iv) Flutax-2-MNs, respectively, in SKOV-3 cell lines, we conducted the following fluorescence imaging experiments.

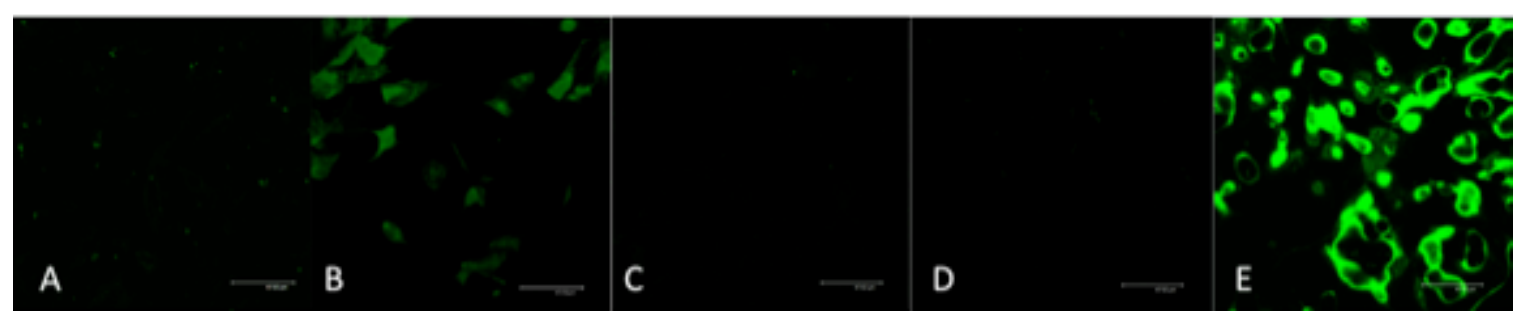

Figure 29: Confocal microscopy imaging of the drug uptake by SKOV-3 with different drug carriers. Confocal microscopy image of SKOV-3 cells showing the sub-localization of Flutax-2 (drug) when bound to different carrier systems: (a) free drug, (b) drug-HER-2, (c) drug-GMO-MNs in 30-Oe field. (d) drug-GMO-MENs with no field, (e) drug-GMO-MENs in 30-Oe field. The scale bar is $47 \mu \mathrm{m}$.

\subsubsection{Field dependence of drug uptake in cancer and healthy cells}

To understand the field dependence of the described process, we performed the cellular drug uptake experiments under a varying magnetic field strength on both cancer ovarian (SKOV-3) and healthy ovarian cell (HOMEC) lines. The HOMEC cells were cultured according to the same procedures that are described for the SKOV-3 cells in Section Methods. As a control, the cells with GMO-MENs only (without Flutax-2) were treated under the equivalent conditions. The cell culture plates with the MENs and drug-GMOMENs were exposed to three different field strengths, 5, 15, and 30 Oe, respectively. The results are summarized in Figure 30. The measurements showed that, as the field was 
increased above approximately 30 Oe, the drug penetration into the cancer cells (SKOV3) greatly increased. On the other hand, it can be noted that the drug barely affected the healthy cells (HOMEC) in the field range under study.

A.

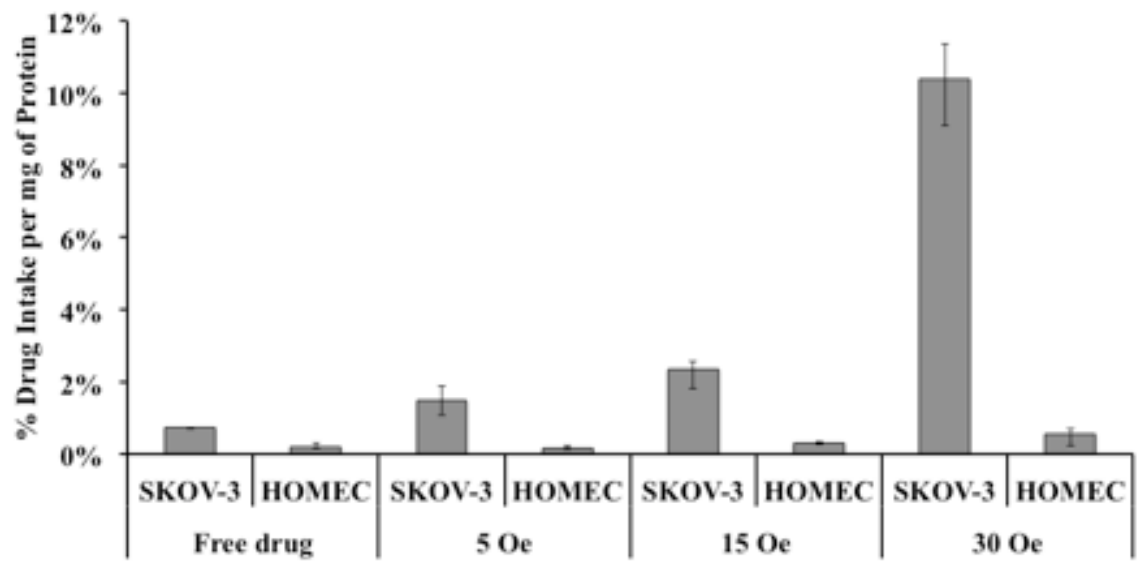

\section{SKOV-3}

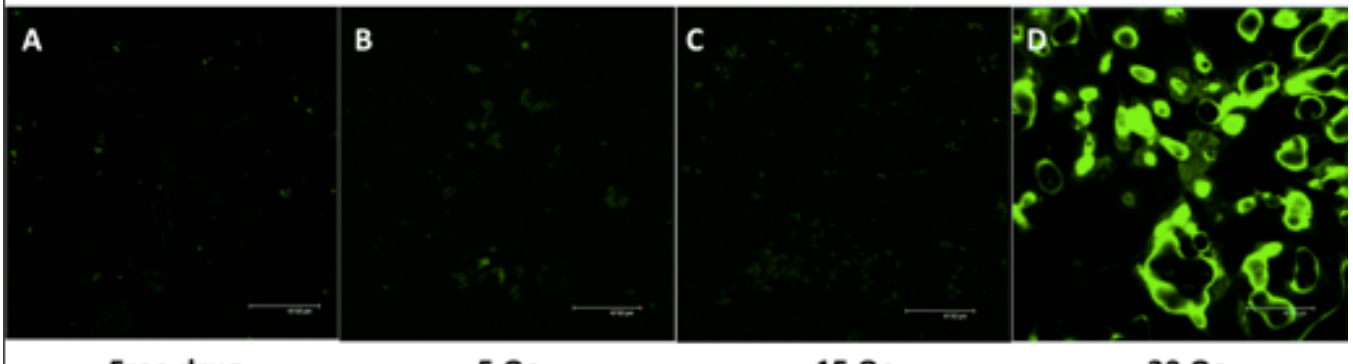

Free drug

5 Oe

15 Oe

30 Oe

HOMEC

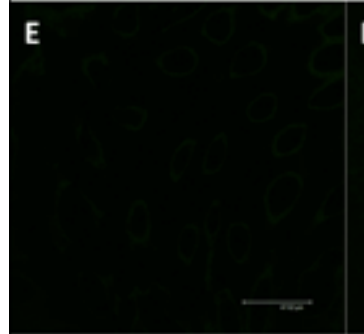

$\mathbf{F}$

G

H

B.

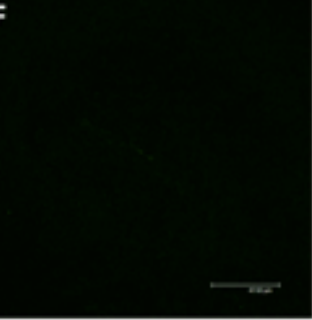

Figure 30: Field dependence of the drug uptake by SKOV-3 and HOMEC cells. Flutax-2 drug uptake by HOMEC and SKOV-3 cells in varying magnetic fields (B). The drug uptake quantitatively presented in (A) was measured by a fluoremeter $(n=3)$. The scale bar on the images is $47 \mu \mathrm{m}$. 


\subsubsection{Cancer cell viability test}

After we confirmed that the drug-loaded MENs in the vicinity of the cancer cells indeed acted, as a field-controlled valve to let the drug in (due to the effective nanoelectroporation effect according to our hypothesis), we studied the viability of the cancer cells for different combinations of the drug and the carrier after the drug penetrated through the cell membrane. (Here, maintaining the remote field at 30 Oe provided the specificity to the cancer cells or, in other words, ensured that the healthy cells were intact.) The summarizing images that were obtained in 24 hours after the penetration are shown in Figure 31. The three key combinations of the carrier included (i) no particle, (ii) HER-2-GMO-MENs (Note: Here, HER-2 stands for the HER-2 biomarker antibody), and (iii) GMO-MENs, respectively. Accordingly, the three images (from left to right) in Figure 31A show the morphology of the cancer cells after 24-hour treatment by (i) the free drug (with no particle carrier), (ii) drug-HER-2-GMO-MENs with no field applied, and (iii) drug-GMO-MENs in a 30-Oe d.c. field. The three control images in Figure 31B show the morphology of the cancer cells after the 24-hour treatment by the same three combinations of the carrier with no drug present. To account for the number of cells viable after the 24-hour treatment, Trypan-Blue viability assay was performed. The quantitative data of the cell viability tests are also summarized in Table 7. 


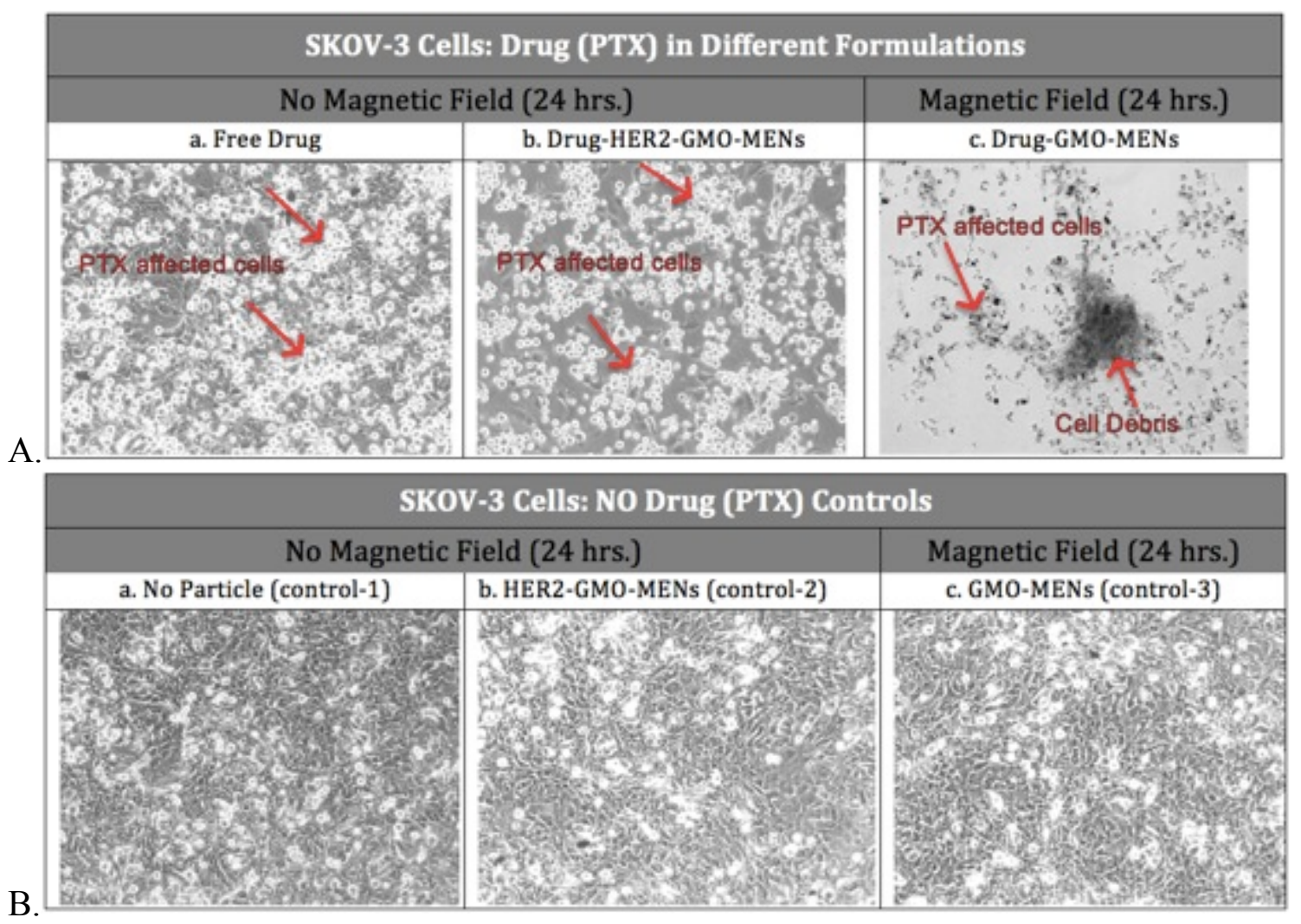

Figure 31: Confocal imaging of SKOV-3 cell viability after treatment by different drug-carrier combinations with and without field. Confocal images of: (A) SKOV-3 Cells treated with PTX drug with different carrier combinations, (left) no career with no field, (middle) HER2-GMO-MENs with no field, and (right) GMO-MENs in a 30-Oe field and (B) SKOV-3 Cell Controls that include the same three carrier combinations with no PTX drug present

Table 7: The table shows the percentage of cells viable after the 24-hour field-treatment period for different PTX-MEN combinations and field treatment conditions $(n=3)$.

\begin{tabular}{|c|c|c|c|}
\hline \multirow{3}{*}{ Drug } & Field (30 Oe) & Type of Treatment & Percentage Cells Alive \\
\hline \multirow{3}{*}{-} & - & $\begin{array}{c}\text { Control-1 } \\
\text { (No particle) }\end{array}$ & $99 \pm 1 \%$ \\
\cline { 2 - 4 } & - & $\begin{array}{c}\text { Control-2 } \\
\text { (GMO-MENs-HER2) }\end{array}$ & $98 \pm 1 \%$ \\
\hline \multirow{2}{*}{+} & + & $\begin{array}{c}\text { Control-3 } \\
\text { (GMO-MENs) }\end{array}$ & $98.5 \pm 0.7 \%$ \\
\cline { 2 - 4 } & - & FREE & $86 \pm 8.8 \%$ \\
\cline { 2 - 4 } & + & GMO-MENS-HER2 & $71 \pm 9.8 \%$ \\
\hline
\end{tabular}




\subsubsection{In-vitro Cytotoxicity Assay}

To determine the cytotoxicity of the GMO-MENs on SKOV-3 cells, a quantitative colorimetric XTT (sodium 2,3,-bis(2-methoxy-4-nitro-5-sulfophenyl)-5-[(phenylamino)carbonyl]-2H-tetrazolium inner salt) assay was performed. The assay is based on the reduction of XTT tetrazolium salt by the viable cells to form orange colored formazan derivative. In this assay, $1 \times 10^{5}$ cells were seeded per well in a 96-well plate and incubated at $37^{\circ} \mathrm{C}$ for 24 hours. After the incubation, the cell medium was replaced by the medium containing the GMO-MENs at a differential concentration of $0-100 \mu \mathrm{g} / \mathrm{ml}$ per well and the cells were incubated for another 24-hour period. Then, the cell medium was replaced with a fresh one and washed with the PBS buffer and cell viability assay was performed by adding $50 \mu \mathrm{l}$ per well of XTT-activated solution from the XTT test kit supplied by ATCC and incubate for 4 hours at $37^{\circ} \mathrm{C}$. The experiments were performed in triplicates. As summarized in Figure 32, no significant cytotoxicity was observed.

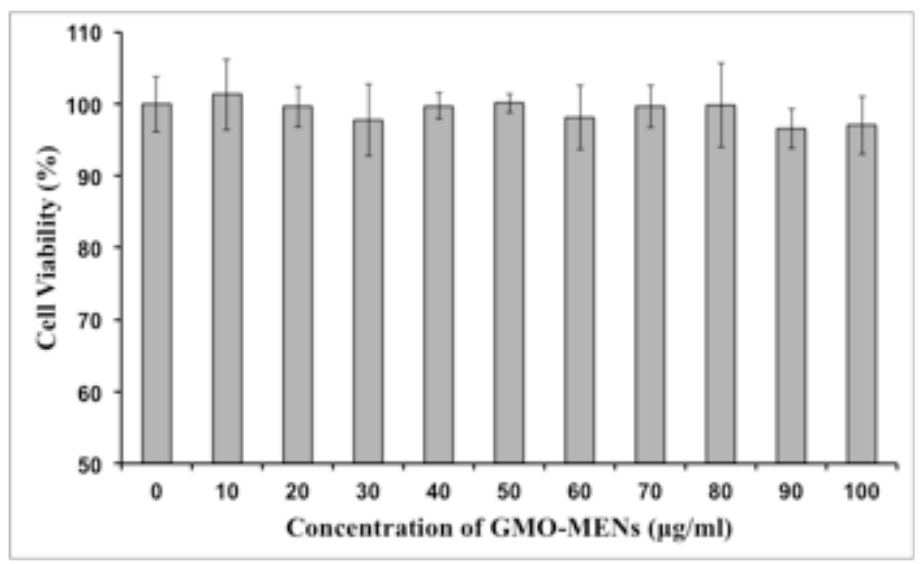

Figure 32: XTT cytotoxicity assay. The chart shows the results of XTT Assay performed on SKOV-3 cells at different concentrations of GMO-MENs $(n=3)$. 


\subsubsection{Heat-dissipation due to field-treatment with MENs}

In this experiment, the temperature was measured locally via Infra-red (IR) camera FLIRi3 on the surface of both cancer (SKOV-3) and healthy (HOMEC) ovarian cells before and after a field treatment, as shown in Figure 33. The experimental error of the setup was approximately $+/-2$ degree Celsius of the infrared camera. The magnetic field of 30 Oe was applied for a 24-hour period. No significant heat dissipation was observed, as a result of the field treatment. The negligible heat dissipation (compared to the conventional method) is a direct consequence of the intrinsic nature of the magnetoelectric coupling which resulted in the unprecedented high-efficacy control of intrinsic electric fields by external magnetic fields.

A. SKOV-3 before and after $\mathrm{H}$
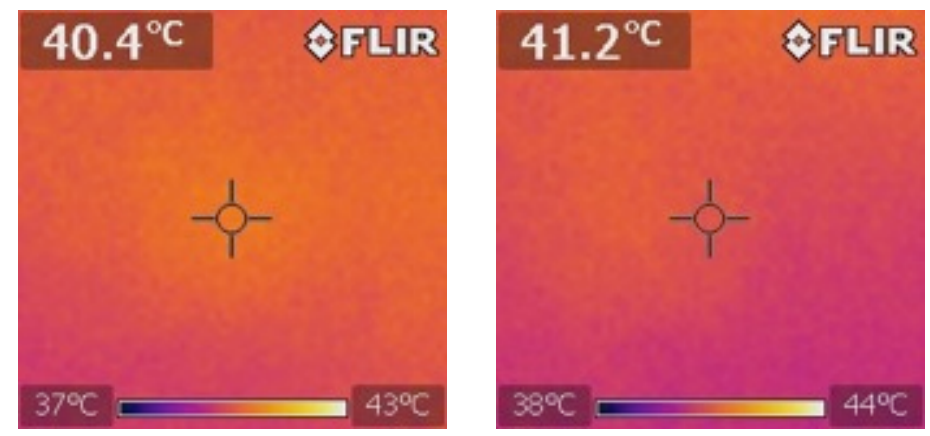

B. HOMEC before and after $\mathrm{H}$
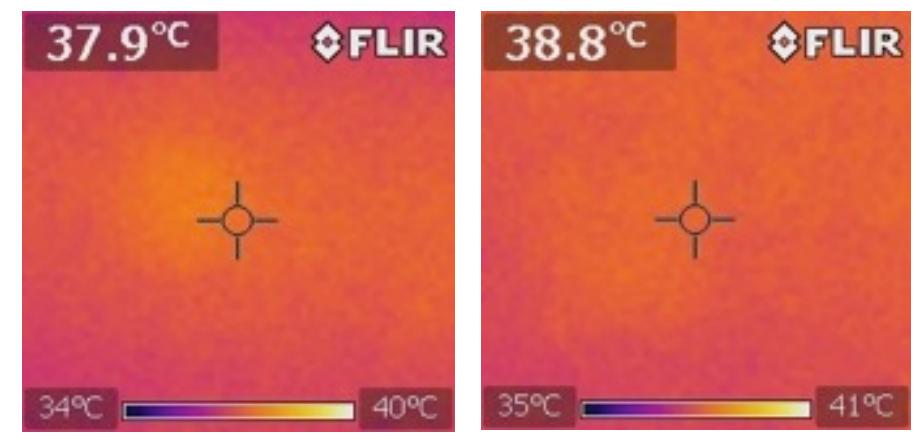

Figure 33: Dissipation-free physics. Surface temperature measured via Infra-red (IR) light for (A) cancerous (SKOV-3) and (B) healthy (HOMEC) ovarian cells before and 100-Oe field treatment 


\subsection{Discussion}

The results of the above experiments confirmed our hypothesis that MENs loaded with the drug PTX could serve, as high-specificity remotely controlled (via magnetic fields) delivery nanosystems to treat EOC. This function was achieved due to the localized electroporation effects induced by the MENs in the vicinity of the cancer cell membranes when exposed to an external magnetic field. The experiments were conducted to separate the two core field-dependent processes according to the main hypothesis. These two processes are defined by the following two critical fields, respectively: (i) the threshold field, $\mathrm{H}_{\text {th }}$, for MENs to penetrate through the cancer cell membrane to deliver the drug into the cell cytosol (by means of the field-induced localized nano-electroporation effect in the vicinity of MENs); and (ii) the release field, $\mathrm{H}_{\mathrm{r}}$, that triggers unloading of the drug after the drug-loaded MENs penetrated into the cell. The specificity to the cancer cells was defined not just by the typical HER-2 antibody chemistry, but also by the physical mechanism that relied on the significant difference in the threshold field between the healthy and cancer cells. This threshold field was measured to be of the order of $30 \mathrm{Oe}$ and above 200 Oe for the SKOV-3 and HOMEC cells, respectively. Moreover, these experiments indicated that this remote-magnetic-field-triggered electric-field-defined specificity to the cancer cells resulted in a more pronounced eradication of the cancerous cells. The percentage of the cell-penetrated drug was increased by at least a factor of five compared to the traditional antibody-mediated targeting (Figure 29). In addition, after the drug was efficiently transferred through the tumor cell membrane by the field-controlled MEN-initiated nano-electroporation, complete eradication of the cancer cells (without 
affecting the healthy cells) was observed within a 24-hour period of a low-energy 30-Oe treatment (Figure 30 and Figure 31).

To achieve adequately high efficacy of the drug delivery, the value of the release field, $\mathrm{H}_{\mathrm{r}},(\sim 30 \mathrm{Oe})$ was chosen to be higher than the value of the threshold field to penetrate through the membrane, $\mathrm{H}_{\mathrm{th}},(\sim 10 \mathrm{Oe})$ for the cell of the same type. It can be reminded that the release field is defined by the binding force between the MEN and the drug, while the penetration threshold field, $\mathrm{H}_{\mathrm{th}}$, is mostly determined by the electric properties of the cell membrane that lead to the localized electroporation effects. We could control the release field by the proper selection of the intermediate layer between the drug and the MEN. (As summarized in Figure 36, by choosing different intermediate layers we could control the initial release field in a wide range, from less than 10 Oe to over 200 Oe. By default, a 2-nm thin GMO layer was used, as the intermediate layer.) In addition, the release field depended on the field treatment duration and the frequency of the a.c. field, as shown in Figure 23B. For example, as shown in Figure 23A, the spectrophotometry measurements of the absorbance at $230 \mathrm{~nm}$ (for PTX) indicated that only 1 minute of field treatment at a 66-Oe d.c. magnetic field was sufficient to release over $95 \%$ of the drug. As shown in Figure 23B, the same release efficacy (of over $95 \%$ ) could be achieved also at a 1000-Hz a.c. field at smaller field strength of 44 Oe in 2 hours of treatment. This complex dependence can be explained by the fact the external field effectively reduces the energy barrier that holds together the MEN and the drug while an increase of the treatment duration increases the temperature-induced probability to overcome the barrier or, in other words, break the bond. As for the frequency 
dependence, in our previous paper we explained the underlying physics through field torque effects that break the bond,, the frequency increases (1). Here, it can be mentioned that although using a.c. external magnetic fields could indeed add another knob to control the targeted delivery, in this study to focus on the proof of the main hypothesis, as illustrated in Figure 18, we followed the d.c. field scenario. For example, a 3-angstrom thin GMO as the intermediate layer resulted in the d.c. release field to be of the order of 30 Oe for the 24-hour treatment period. The d.c.-field-controlled drug release kinetics was confirmed also through AFM, FTIR, and Mass Spectrometry, and X-ray diffraction pattern studies, as shown in Figure 24, Figure 25, Figure 26, and Figure 27, respectively. As confirmed by infrared measurements of the cellular surface temperature (Figure 33), the MENs's field action didn't trigger any significant temperature changes in the field and frequency range under study. This is in agreement with the fact that the MENsinduced delivery is an extremely energy-efficient process (because of the strong quantum-mechanically determined magneto-electric coupling), which causes only negligible heat dissipation.

\subsection{Conclusion}

As for the penetration threshold field, $\mathrm{H}_{\mathrm{th}}$, we found that for the cancer cell membrane the value was of the order of 30 Oe, i.e. less than the d.c. release field (for the default MEN carriers coated with GMO) of 60 Oe. Again, according to the main hypothesis, to ensure the specificity to the cancer cells, it is important to maintain the remote field above the release value for the tumor cells but lower than the threshold value for the healthy cells. 
Indeed, at a 30-Oe external d.c. field, the drug couldn't penetrate through the healthy cell membrane for the 24-hour treatment duration, which confirmed that the threshold field for the drug-loaded MENs to penetrate into the healthy cell exceeded 30 Oe during the entire treatment (Figure 30). Specifically, the GMO-MENs field-treated HOMEC cells showed a negligible drug intake per $1 \mathrm{mg}$ of the cellular protein content. The value was $0.18 \pm 0.07,0.30 \pm 0.04$, and $0.55 \pm 0.16 \%$ for the field strength of 5,15 , and $30 \mathrm{Oe}$, respectively. On the contrary, SKOV-3 cells showed significantly higher values of the drug intake, which was $1.50 \pm 0.41,2.36 \pm 0.48$, and $10.41 \pm 1.54 \%$ for the field strength of 5,15 , and 30 Oe, respectively.

Least but not last, the cytotoxicity measurements with the standard XTT assay performed on SKOV-3 cells at different concentration of MENs showed no significant toxicity even at the highest nanoparticles concentration value of $100 \mu \mathrm{g} / \mathrm{ml}$ (Figure 32). The chart shows the results of XTT Assay performed on SKOV-3 cells at different concentrations of GMO-MENs.

Note: How to cite this chapter: The content of this chapter is published in my Nature Scientific Reports article, hence please use this as a reference: Guduru, Rakesh, Ping Liang, Carolyn Runowicz, Madhavan Nair, Venkata Atluri, and Sakhrat Khizroev. "Magneto-electric Nanoparticles to Enable Field-controlled High-Specificity Drug Delivery to Eradicate Ovarian Cancer Cells." Scientific reports 3 (2013). 
Chapter 4: Functionalized Magneto-electric Nanoparticles for Controlling the Drugreleasing Threshold Field 


\subsection{Introduction}

Over the last decade, nanomaterials have been widely used to deliver the therapeutics drugs to the diseased tissue in order to increase the bioavailability and to reduce the toxic side effects to the healthy tissues (132-134). Using surface modification to further tailor important characteristics of the nanomaterials is a promising approach to develop future applications in medicine, biology, and biotechnology (135-138). In the field of drug delivery, surface-modification of nanomaterials plays a crucial role in determining drug binding \& release kinetics $(139,140)$. Commonly used surface-functionalizing agents to coat nanomaterials include poly(lactic-co-glycolic acid) (PLGA), glycerol monooleate (GMO), Poly-L-Lysine, Tween-20, and Ethyl-3-(3-dimethylaminopropyl) carbodiimide (EDC) (141-145). Drug delivery and release using most of these surfacefunctionalized nanomaterials are typically governed by the change in pathological conditions, such as $\mathrm{pH}$, temperature, redox microenvironments, and enzymatic reactions (146-149). Consequently, the capability to control the delivery and particularly release processes in a wide range of properties suited for different applications remains an important challenge. Alternatively, the recently introduced body-temperature magnetoelectric nanoparticles (MENs) exploit the magneto-electric (ME) effect to release the therapeutic drug cargo without compromising the structural and functional integrity of the drug at body temperature (1). The ME effect allows to use an external magnetic field to remotely access the intrinsic electric fields that govern the binding forces between the MEN's surface and the drug load. The ability to remotely control this binding force would be an important milestone in the development of nanostructures for controlled targeted drug delivery and release. Previously, we have shown that there is a certain 
frequency-dependent threshold magnetic field for the drug to be released. (Thereafter we refer to this field also, as the dissociation field.) This field is determined by the ionic and/or covalent interaction between the two bonded entities or, in other words, by the Coulomb's forces between the oppositely charged MEN and the drug molecules in the bond (polarized due to the chemical interaction). Therefore, this dissociation field can be strongly influenced by a thin $(\sim 2 \mathrm{~nm})$ intermediate layer, which changes the polarization of the two entities. Therefore, we refer to this intermediate layer also, as the functionalization layer. The important goal of the described study is to demonstrate how tailoring the composition of the intermediate layer could be used to control the dissociation field magnitude and frequency, specific for each application. Particularly, experiments were developed to dissociate the drug molecules from the MENs' surface at the following three different ranges of the remote magnetic fields, (i) low ( $<10 \mathrm{Oe})$, (ii) moderate (100 Oe), and (iii) high ( $>200 \mathrm{Oe})$, respectively, in a frequency range from 0 to over $1000 \mathrm{~Hz}$. These field ranges are relevant for many important applications, e.g., treatment of HIV in the brain, central nervous system (CNS) stimulation, deep-tissue drug delivery for cancer treatment, magnetic imaging, and others. Further, the control via the field frequency provides an additional knob particularly important for the release process (1). Introducing the intermediate functionalization layer will result in changing the binding affinity between the drug and the MEN's surface. One important requirement to this layer is to maintain sufficiently strong binding with the MEN's surface while keep binding to the drug molecules relatively weak. Accordingly, we implemented this concept by choosing the following three intermediate layers, (i) Glycerol monooleate (GMO), (ii) Tween-20, and (iii) Ethyl-3-(3-dimethylaminopropyl)-carbodiimide (EDC), 
respectively. In this study, we used Paclitaxel (PTX) as the model drug, as it is one of the most widely used antineoplastic agents against ovarian, breast, neck, head and non-smallcell lung carcinoma, and others $(120,150,151)$. We used $\mathrm{CoFe}_{2} \mathrm{O}_{4}-\mathrm{BaTiO}_{3}$ coreshell MENs, as a drug delivery vehicle, due to their ability to exhibit magneto-electric effect at body temperature $(152,153)$.

\subsection{Experimental methods}

\subsubsection{MENs fabrication}

$\mathrm{CoFe}_{2} \mathrm{O}_{4}-\mathrm{BaTiO}_{3}$ coreshell nanoparticles are fabricated according to the procedure described in section 2.2.1

\subsubsection{MENs surface functionalization}

GMO-MENs were prepared by incubating $0.1 \mathrm{mg}$ of GMO with $5 \mathrm{mg}$ of MENs in $5 \mathrm{ml}$ of PBS ( $\mathrm{pH} 7.4$ ) buffer for 12 hrs. (Note: to achieve uniform surface modification, the solution was slowly agitated during incubation). The solution was later subjected to centrifugation at $20,000 \mathrm{rpm}$ for 20 minutes at $10^{\circ} \mathrm{C}$ to remove excess GMO. The obtained pellet was re-suspended in ethyl acetate: acetone (70:30) solution and recentrifuged three times to obtain GMO-MENs. Tween-20-MENs were prepared by incubating $0.1 \mathrm{mg}$ of Tween-20 with $5 \mathrm{mg}$ of the MENs in $1 \mathrm{ml} \mathrm{PBS}(\mathrm{pH} 7.4$ ) buffer for 2 hrs. while agitating slowly. Later, the solution was centrifuged at 20,000 rpm for 20 minutes at $10^{\circ} \mathrm{C}$ to remove excess Tween-20. The previous step was repeated thrice to obtain Tween-20-MENs. EDC-MENs were prepared by incubating $125 \mu \mathrm{l}$ of EDC (conc. $1 \mathrm{mg} / \mathrm{ml}$ in PBS) with $5 \mathrm{mg}$ of the MENs in $5 \mathrm{ml}$ of PBS (pH 7.4) buffer for 4 hrs. while 
agitating slowly. Later, the solution was centrifuged at $20,000 \mathrm{rpm}$ for 20 minutes at $10^{\circ}$ C to remove excess EDC. The previous step was repeated thrice to obtain EDC-MENs. Surface modified MENs were lyophilized and stored at $4^{\circ} \mathrm{C}$ until further use.

\subsubsection{PTX drug loading}

$100 \mu \mathrm{g}$ of PTX drug was added to $1 \mathrm{ml}$ PBS (pH 7.4) buffer containing $1 \mathrm{mg}$ of desired nanoparticles (MENs, GMO-MENs, Tween-20-MENs, and EDC-MENs). The solution was later incubated for $3 \mathrm{hrs}$. while agitating slowly at room temperature. After incubation, the solution was centrifuged at $3000 \mathrm{rpm}$ for 10 minutes at $10^{\circ} \mathrm{C}$ to collect the pellet containing drug loaded nanoparticles. The separated supernatant was isolated and measured for absorbance spectrophotometrically (Cary-100 UV-VIS) at $230 \mathrm{~nm}$ to determine the unbounded drug (154). Figure 34 shows the PTX standard calibration plot obtained by varying the drug amount (50 $\mu \mathrm{g}$ to $3.25 \mu \mathrm{g}$ ) in $1 \mathrm{ml}$ of PBS buffer. The drugloading percentage was calculated using the below equation.

$$
\text { Drug loading }(\%)=(\text { total amount of drug added }- \text { amount of drug in the supernatant }) \times 100
$$
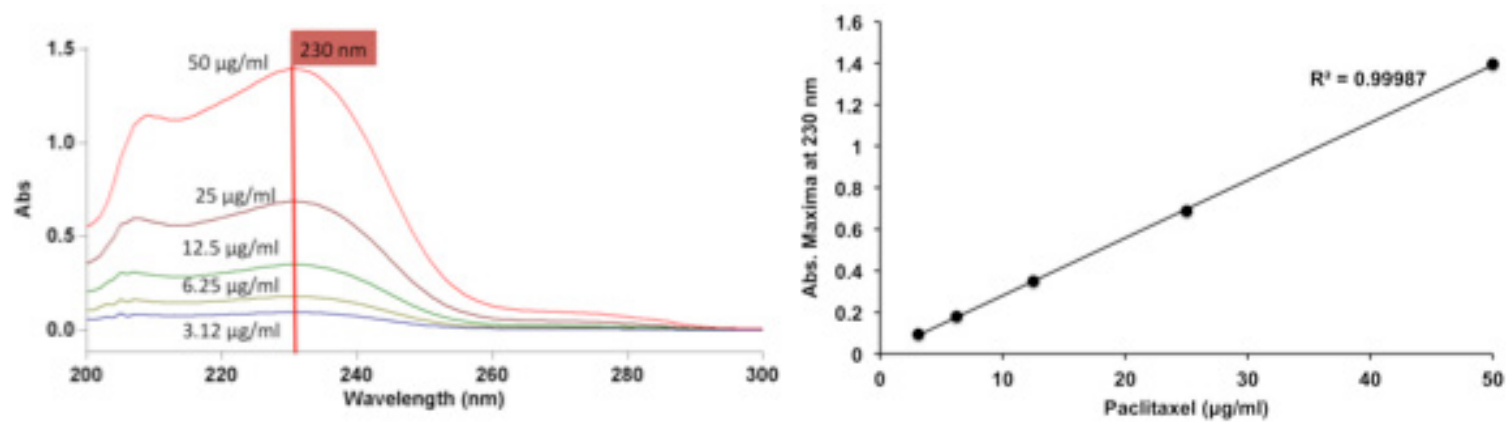

Figure 34: Paclitaxel drug calibration curve obtained using spectrophotometer. A) Absorption maxima at 230 $\mathrm{nm}$ for different drug concentration $(50 \mu \mathrm{g} / \mathrm{ml}$ to $3.12 \mu \mathrm{g} / \mathrm{ml})$ in PBS buffer. B) Linear dependence of $230 \mathrm{~nm}$ absorption maxima with the drug concentration. 


\subsubsection{Remote magnetic field triggered PTX drug release}

The isolated pellet after drug loading was washed twice with the PBS (pH 7.4) buffer to ensure the complete removal of the residual/unbounded drug. Then, the pellet was resuspended in $1 \mathrm{ml}$ of PBS buffer and subjected to varying magnetic field strengths and frequencies. The magnetic fields were generated using low-powered Helmholtz coils connected to a d.c. power supply of $0 \mathrm{~Hz}$ and to an a.c. generator of $100 \mathrm{~Hz}$ and $1000 \mathrm{~Hz}$. After every single treatment, the solution was subjected to centrifugation at $3000 \mathrm{rpm}$ for 3 minutes to separate the supernatant. This supernatant was used to measure the absorbance spectrophometrically at $230 \mathrm{~nm}$ to account for the amount of released drug. (Note: the setup of the magnetic field treatment is shown in Figure 8)

\subsection{5. $S K O V-3$ Cell culture}

Human ovarian carcinoma cell lines (SKOV-3) were used to investigate the in vitro cytotoxicity of the nanoparticles (MENs, GMO-MENs, Tween-20-MENs, and EDCMENs). SKOV-3 cells were purchased from American Type Culture Collection (Manassas, Virginia). McCoy's 5A medium (Life Technologies, NY) supplemented with $10 \%$ fetal bovine serum (Sigma-Alrich) and 1\% penicillin-streptomycin (science-cell) was used, as a cell culture medium. SKOV-3 cells were cultured in an incubator at $37^{\circ} \mathrm{C}$ temperature, $5 \% \mathrm{CO}_{2}$ and humidified atmosphere. 


\subsubsection{In-vitro Cytotoxicity measurements}

The cytotoxicity of the MENs, GMO-MENs, Tween-20-MENs, and EDC-MENs on SKOV-3 cells was measured using a quantitative colorimetric XTT (sodium 2,3,-bis(2methoxy-4-nitro-5-sulfophenyl)-5-[(phenylamino)-carbonyl]-2H-tetrazolium inner salt) assay (155). Briefly, approximately 0.1 million SKOV-3 cells were seeded per well in a 96-well cell culture plate along with a $100 \mu \mathrm{l}$ of growth medium. Cell culture plate was incubated for $24 \mathrm{hrs}$. at $37^{\circ} \mathrm{C}$ to reach confluency. Then, the growth medium was replaced with the medium containing desired nanoparticles at varying concentration 0 $100 \mu \mathrm{g} / \mathrm{ml}$ per well and allowed to incubate for another $24 \mathrm{hrs}$. After $24 \mathrm{hr}$. incubation, growth medium containing nanoparticles was removed and cells were washed with PBS buffer twice to remove the dead cells. Now, $100 \mu \mathrm{l}$ of the fresh medium along with a 50 $\mu \mathrm{l}$ of XTT-activated solution (from the XTT test kit supplied by ATCC) was added to each well containing the cells and was returned to the incubator for 4 more hours. (Note: XTT assay results presented were the average of three independent measurments).

\subsubsection{MENs size and surface charge measurements}

Size and surface charge measurements of the nanoparticles were performed using Malvern Zetasizer (156). $0.5 \mathrm{mg}$ of the desired nanoparticles (MENs, GMO-MENs, Tween-20-MENs, and EDC-MENs) was dispersed in $1 \mathrm{ml}$ of D.I water and this solution was used to measure the size (in standard cuvette) and charge (in folded capillary cuvette). (Note: The size and charge data presented in the results is the average of three independent measurements). 


\subsubsection{Sample preparation for FT-IR measurements}

One drop (10 $\mu$ l) of the desired nanoparticle solution (MENs, GMO-MENs, Tween-20MENs, and EDC-MENs) at concentration $1 \mathrm{mg} / \mathrm{ml}$ was taken on a pre-cleaned silicon wafer and dried over night to measure the FT-IR spectrum. All the FT-IR measurements were performed using JASCO-4100 instrument.

\subsection{Results}

\subsubsection{MEN's Intermediate (Functionalization) Layer}

As mentioned above, in order to tailor the dissociation field magnitude and frequency required to trigger the release of the bounded PTX from MENs in a specific application, we coated the MENs' surface with the following three intermediate layers, GMO, Tween20, and EDC, respectively, as shown in Figure 35. By its nature, Zeta-potential indirectly reflects the binding affinity between a nanomaterial and the drug. The higher Zeta potential implies the higher effective charge and therefore results in the higher Coulomb interaction and the higher dissociation field. We measured the surface zeta-potential, size, and Fourier Transform Infra-Red (FTIR) absorption spectrum of MENs before and after functionalization to understand the effects of the selected surface modification. Indeed, as summarized in Table 8 , the results show a significant dependence of the zeta potential values on the functionalized material (average of three independent measurements): -45.0 $\mathrm{mV} \pm 1.72$ for non-functionalized MENs, $-41.6 \mathrm{mV} \pm 0.26$ for GMO-MENs, $-34.9 \mathrm{mV} \pm$ 0.20 for Tween-20-MEN, and $-30.4 \mathrm{mV} \pm 0.81$ for EDC-MENs. The results also indicate a slight $(\sim 3 \%)$ increase in the average size $(n=3)$ of MENs after coating with the 
functionalization layer: $28.6 \mathrm{~nm} \pm 7.5$ for MENs, $30.9 \mathrm{~nm} \pm 8.9$ for GMO modified MENs, $29.5 \mathrm{~nm} \pm 5.6$ for Tween-20 modified MENs, and $31.1 \mathrm{~nm} \pm 4.4$ for EDCmodified MENs. FTIR spectrum of the GMO modified MENs surface (Figure 36B) showed peaks at $1738 \mathrm{~cm}^{-1}$ and $2941 \mathrm{~cm}^{-1}$, which correspond to the ester bond, and $\mathrm{CH}_{2}$ stretching modes, respectively. The peaks indicate the presence of the GMO layer on the MENs surface (143). The Tween-20 modified MEN's surface (Figure 36C) showed FTIR peaks at $1158 \mathrm{~cm}^{-1}$ and $2862 \mathrm{~cm}^{-1}$ that correspond to $\mathrm{C}-\mathrm{O}$ ester bonds and $\mathrm{C}-\mathrm{H}$ bonds, respectively (157). Figure 36D shows FTIR spectral peaks of the EDC modified MENs at 2852 to $2921 \mathrm{~cm}^{-1}$ and $1460 \mathrm{~cm}^{-1}$, which correspond to a C-H alkanes stretch and amide bonds, respectively (158).

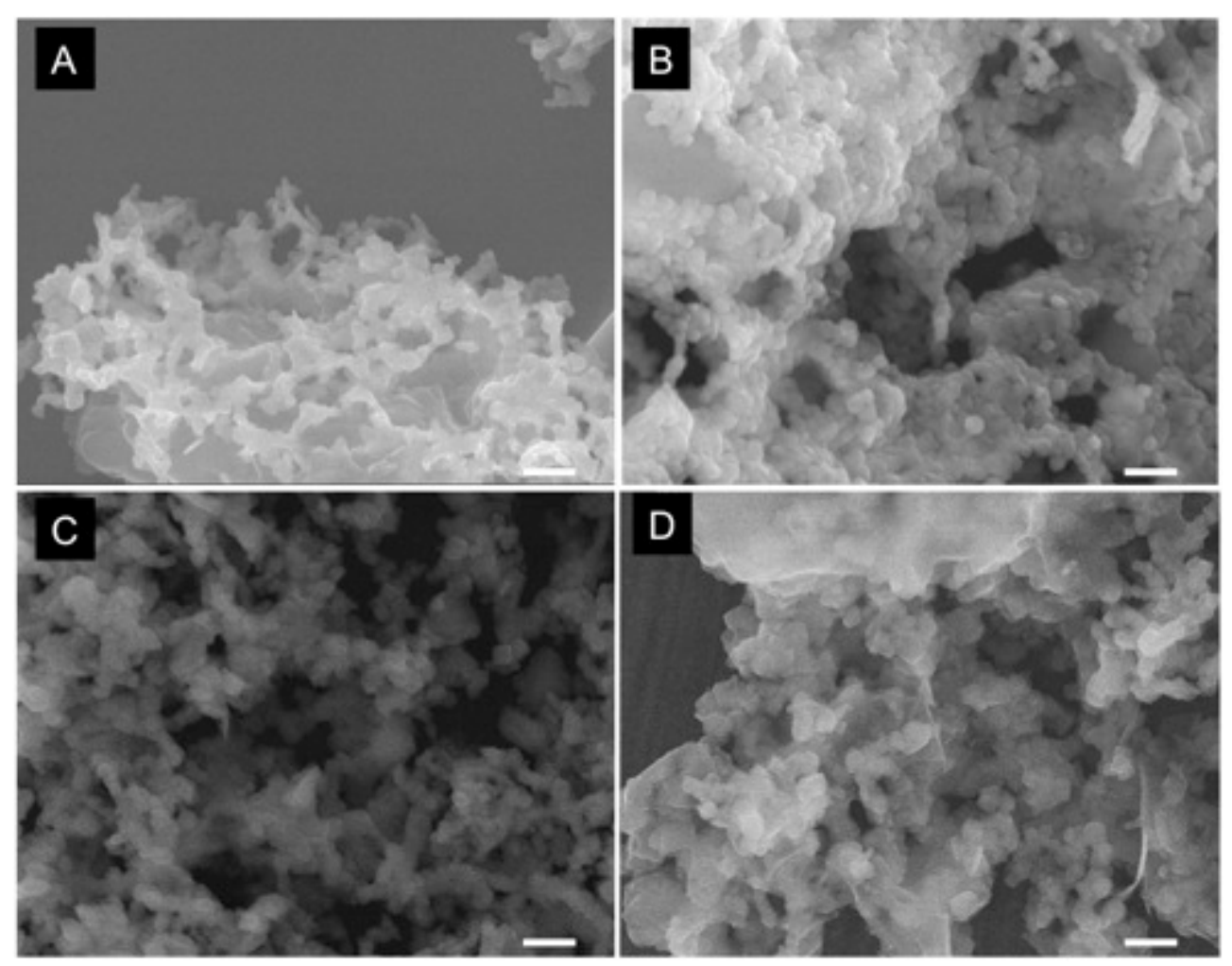

Figure 35: SEM image showing of the MENs. A) Uncoated MENs, B) GMO-MENs C) Tween20-MENs, and D) EDC-MENs. Scale bar 100nm. 
Table 8: Change in zeta-potential and size measurements of the MENs surface before and after surfacefunctionalization. Zeta-potential values are the average and standard deviation of three independent trails $(n=3)$.

\begin{tabular}{lcc}
$\begin{array}{l}\text { Functionalizing } \\
\text { agent }\end{array}$ & $\begin{array}{c}\text { Size } \\
{[\mathrm{nm}]}\end{array}$ & $\begin{array}{c}\text { Zeta potential } \\
{[\mathrm{mV}]}\end{array}$ \\
\hline Uncoated-MENs & $28.6 \pm 7.5$ & $-45.0 \pm 1.72$ \\
GMO-MENs & $30.9 \pm 8.6$ & $-41.6 \pm 0.26$ \\
Tween-20-MENs & $29.5 \pm 5.6$ & $-34.9 \pm 0.20$ \\
EDC-MENs & $31.1 \pm 4.4$ & $-30.4 \pm 0.81$ \\
PTX-MENs & $28.9 \pm 7.7$ & $-44.8 \pm 1.67$ \\
PTX-GMO- & $44 \pm 6.6$ & $-40.7 \pm 0.1$ \\
MENs & & \\
PTX-Tween20- & $32.3 \pm 6.1$ & $-31.1 \pm 0.51$ \\
MENs & & \\
PTX-EDC-MENs & $33.8 \pm 3.2$ & $-27.7 \pm 1.3$ \\
\hline
\end{tabular}

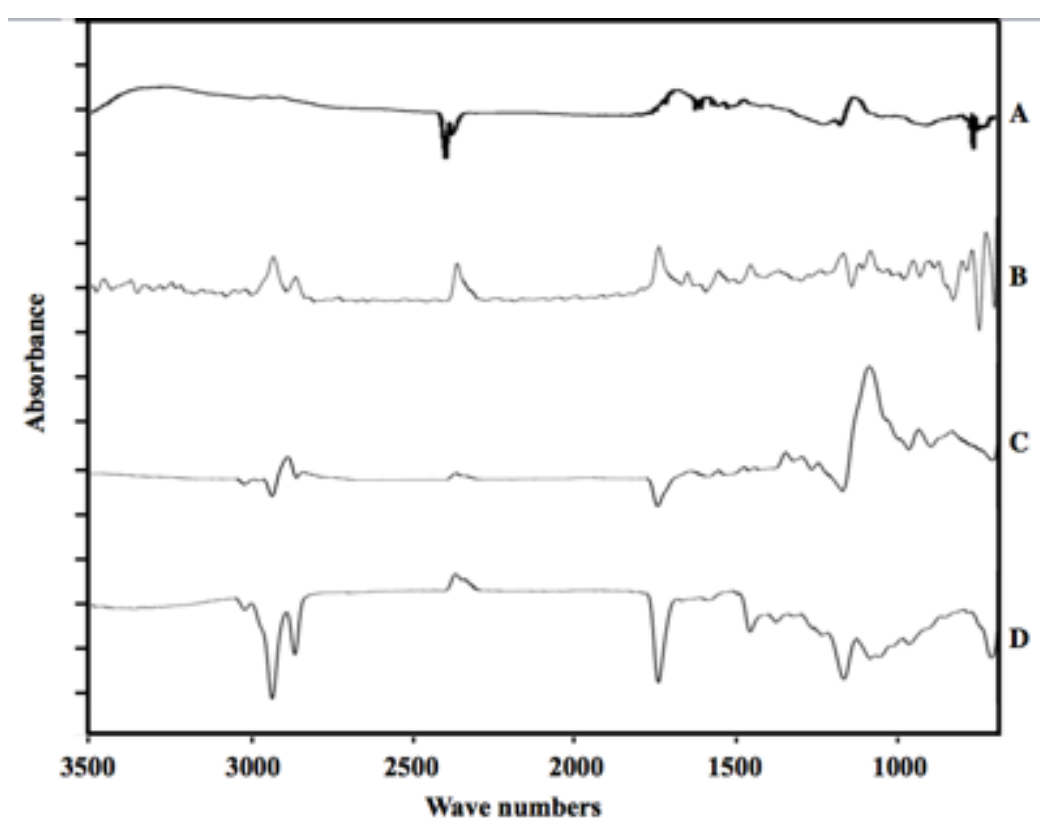

Figure 36: FT-IR for surface functionalized MENs. MENs (A), GMO-MENs (B), Tween-20-MENs (C), and EDC-MENs (D). 


\subsubsection{PTX Drug Loading onto MENs surface}

PTX drug loading onto differently functionalized MENs' surfaces was achieved by incubating the drug with the MENs in PBS buffer ( $\mathrm{pH}$ 7.2). Time-dependent drugbinding kinetics was performed to determine the ideal incubation time to maximize the drug loading percentage. The knowledge of the loading capability of the nano-carrier type (MENs) is important for optimizing its drug delivery characteristics. Figure 37A shows that $\sim 16 \%, \sim 42 \%, \sim 91 \%$, and $\sim 77 \%$ of PTX binds to the uncoated MENs, GMOMENs, Tween20-MENs and EDC-MENs, respectively for $3 \mathrm{hrs}$. of incubation. The drug loading percentage was determined by measuring the UV light absorption maxima at 230 $\mathrm{nm}$ in supernatant solution. Figure 34 shows the PTX absorption maxima at different drug concentrations. To determine the binding efficacy of the PTX drug with different MENs, we performed a binding isotherm study by varying the drug concentration from $25 \mu \mathrm{g} / \mathrm{ml}$ to $200 \mu \mathrm{g} / \mathrm{ml}$ per $1 \mathrm{mg}$ of MENs. As shown in Figure 37B, the results indicate the PTX binding efficacy was highest for EDC-MENs (79 $\mu \mathrm{g}$ to $86 \mu \mathrm{g})$ followed by Tween20-MENs (55 $\mu \mathrm{g}$ to $58 \mu \mathrm{g}$ ), GMO-MENs (34 $\mu \mathrm{g}$ to $39 \mu \mathrm{g}$ ), and the uncoated MENs $(3 \mu \mathrm{g}$ to $4 \mu \mathrm{g})$. To simulate the PTX binding efficacy in the human blood circulatory system, we continuously circulated these particles for 2 hours using a peristaltic pump at a flow rate of $8 \mathrm{ml} /$ minute. The measured percentage of PTX (loaded on MENs) that remained intact, as a result of the circulation experiment was $91.1 \%$ for EDC-MENs, $87.0 \%$ for Tween-20-MENs, $79.5 \%$ for GMO-MENs and $58.4 \%$ for nonfunctionalized MENs. 
A.

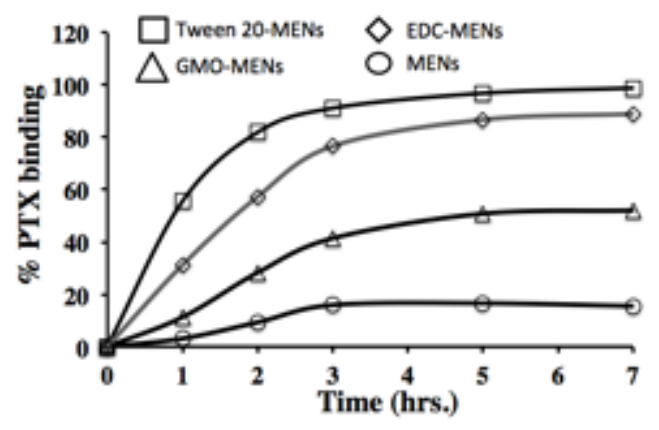

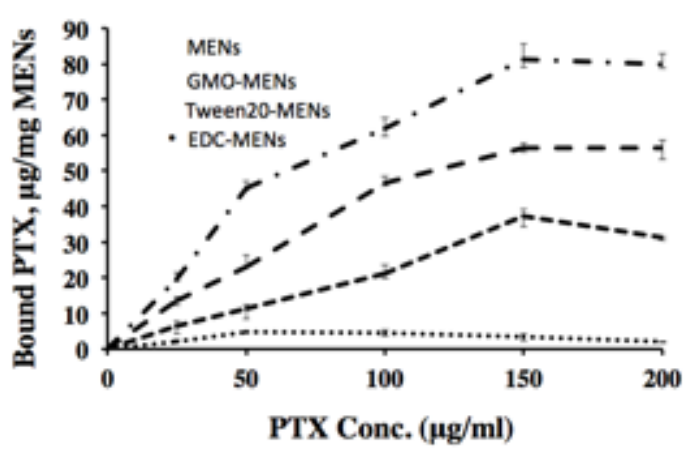

B.

Figure 37: PTX drug loading. A) Time kinetic of PTX binding onto different MENs surface at 1:10 weight ratio (0.1 mg of PTX and $1 \mathrm{mg}$ of MENs). B) Binding isotherm of PTX onto different MENs surface at varying PTX drug concentration and for 3 hrs. incubation time.

\subsubsection{On-Demand Paclitaxel Release Using Remote Magnetic Fields}

PTX bound to different functionalization layers (of MENs) was treated with d.c. and a.c. magnetic fields to release the drug without affecting its functional and structural integrity, according to the procedures described in detail in our previous publication (1). As shown in Figure 38, the results indicated that the threshold magnetic field and frequency required to trigger the release of over $70 \%$ of the drug from the uncoated MENs were approximately $10 \mathrm{Oe}$ and $1000 \mathrm{~Hz}$, respectively. . For GMO-MENs, the two values for releasing over 97\% of PTX became 100 Oe and $0 \mathrm{~Hz}$, respectively. For Tween-20-MENs, to release over $45 \%$ of the drug, the two values were 200 Oe and $1000 \mathrm{~Hz}$, respectively, while for EDC-MENs with the same field and frequency values we could release barely above $5 \%$ of the drug. (Note: due to the limitation of our experimental setup, we could not exceed the field and frequency values above $200 \mathrm{Oe}$ and $1000 \mathrm{~Hz}$, respectively.) 

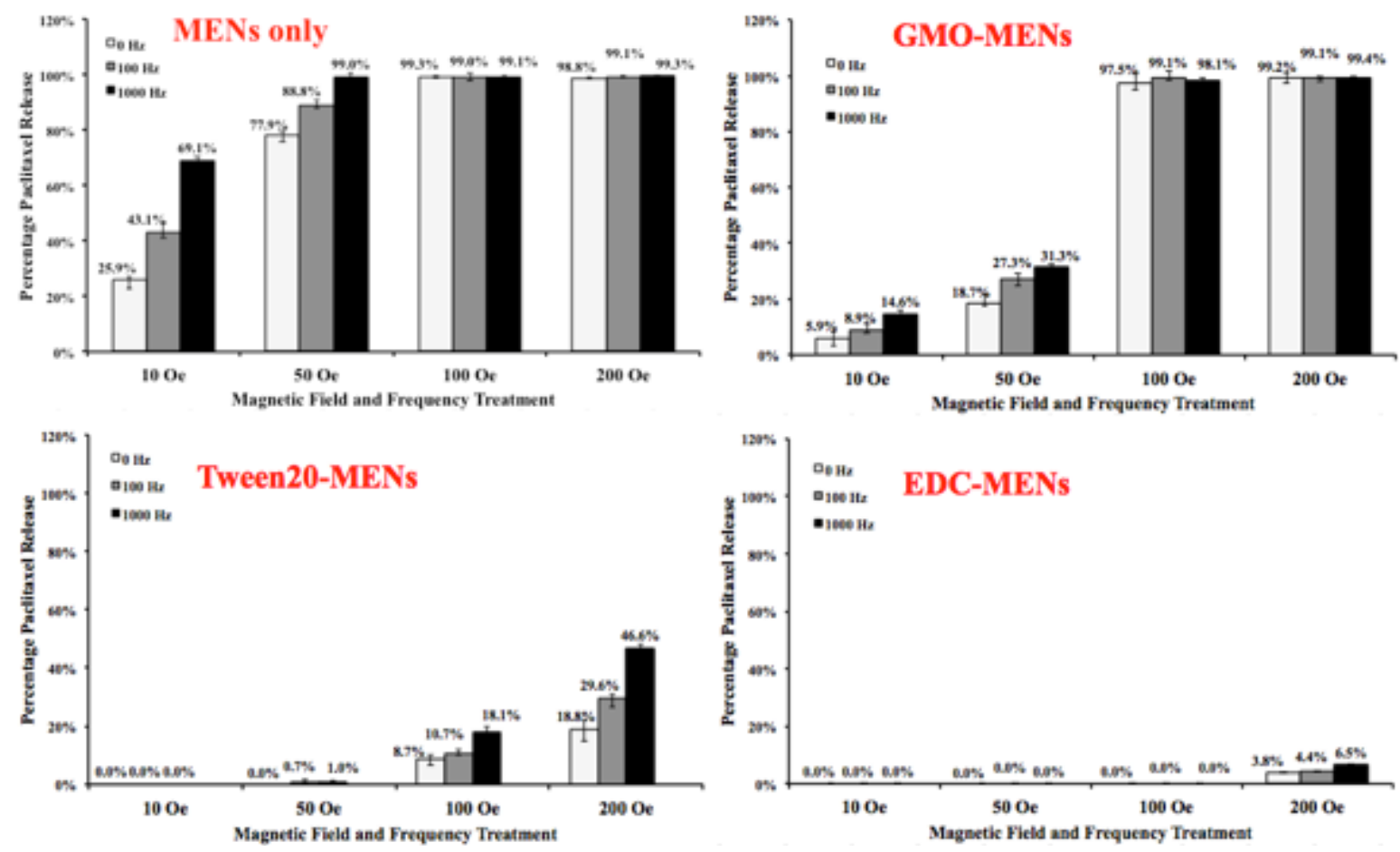

Figure 38: Percentage PTX release at different magnetic field strengths and frequencies. A) Uncoated MENs, B) GMO-MENs, C) Tween20-MENs, and EDC-MENs.

\subsubsection{In-vitro Cytotoxicity}

An in-vitro cytotoxicity study was performed using XTT assay to account for the cytotoxic effects of the surface modified MENs on human ovarian cancer cells (SKOV3). As summarized in Figure 39, the results showed no significant toxicity on SKOV-3 cells when treated with MENs ( $\sim 96 \%$ cells viable), GMO-MENs ( $\sim 100 \%$ cells viable) and Tween-20-MENs ( $\sim 89 \%$ cell viable) at the highest concentration level of $50 \mu \mathrm{g} / \mathrm{ml}$. On the other hand, EDC-MENs showed relatively low toxicity ( $\sim 77 \%$ cells viable) for the same concentration. 
- MENs only 圈 GMO-MENs 困Tween20-MENs $\mathbb{Q}$ EDC-MENs

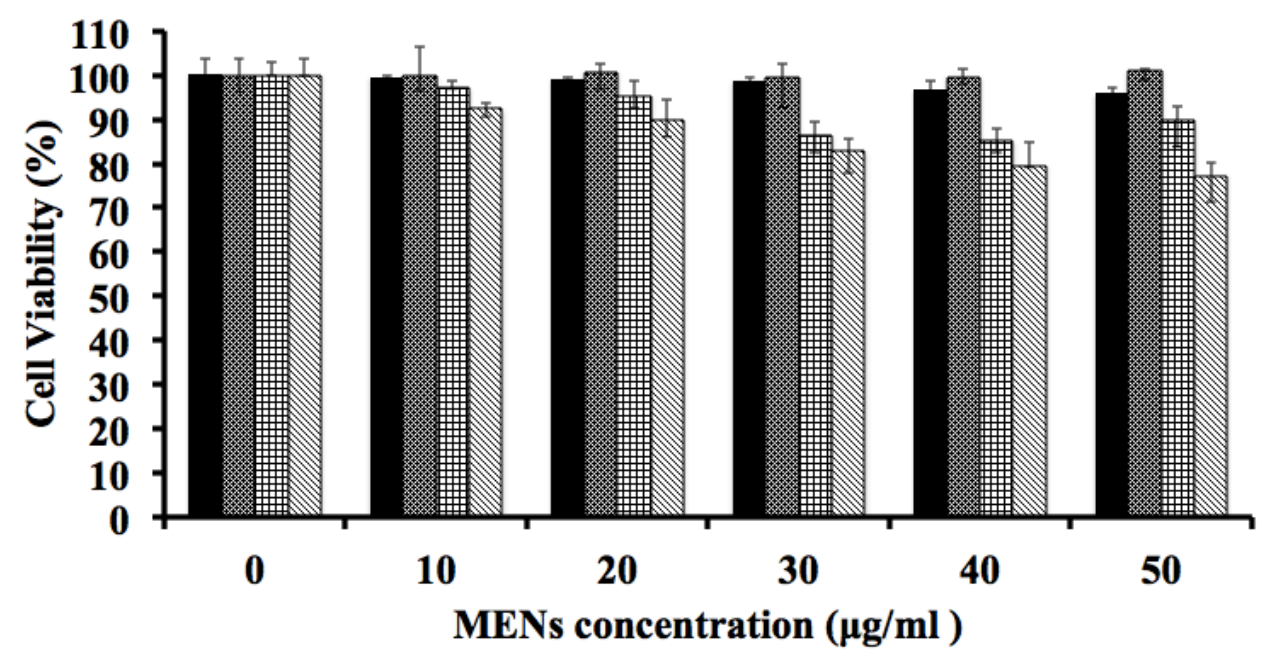

Figure 39: XTT cytotoxicity assay. Viability of the SKOV-3 cells was determined using XTT assay after treating with various concentrations of MENs (uncoated MENs, GMO-MENs, Tween-20-MENs and EDC-MENs) for 48 hrs.

\subsection{Discussion}

Previously we have shown how low-energy external magnetic fields could increase the bioavailability of the therapeutic drug cargo at the site of diseased tissue using the magneto-electric nanoparticles, instead of the conventional magnetic nanoparticles (MNs), as the delivery vehicle thereby overcoming the toxic side effects caused to healthy tissue $(1,16,159)$. One of the important advantages of using MEN-based delivery is the fact that different remote magnetic field modes can be used for delivery and release of the therapeutic payloads with unprecedented energy efficiency that promises widespread application in the future. Exactly, as with the conventional MNs, the drug delivery (navigation through the body) with MENs can be achieved by a d.c. magnetic field gradient with a field magnitude in the range specific to the targeted region 
(superficial or deep-tissue). As for the release process, it cannot be achieved with the conventional MNs and can be achieved with MENs via application of either low-energy a.c. or relatively high d.c. fields. Therefore, unlike the conventional MNs, MENs offer a new capability of field-controlled release. To enable high-efficacy independent control of delivery (navigation) and/or release, MENs allow to separate the respective functions in the magnetic field magnitude and frequency domains, as much as possible. Further, the ability to vary the control fields in the widest possible ranges (in magnitude and frequency domains) can significantly increase the range of potential applications for MENs. For example, it is known that to target deep tissue carcinoma a relatively high d.c. magnetic field (above $100 \mathrm{Oe}$ ) is required for the navigation. On the contrary, superficial drug delivery can be achieved by field gradients with a d.c. field weaker than 100 Oe. Further, the dependence of the release on the field frequency provides another powerful control knob. For example, for GMO-MENs at a $1000-\mathrm{Hz}$ frequency, less than $10 \mathrm{Oe}$ field was sufficient to achieve the same release percentage, as that achieved by the d.c. field at a higher than 100 Oe magnitude. In the current study, we demonstrated how the release fields could be varied in relatively wide magnitude and frequency ranges, from below 10 to over $200 \mathrm{Oe}$ and from 0 to over $1000 \mathrm{~Hz}$, respectively, by choosing the adequate intermediate (functionalization) layer between the MEN's surface and the drug molecules. Specifically, we studied the following three layer types, (i) GMO, (ii) Tween20, and (iii) EDC, respectively. As mentioned above, the navigation by MENs is similar to the navigation by the conventional MNs while the field-controlled drug release is specific to the MEN-based delivery only. Therefore, this study focused on the release kinetics defined. Previously, we have shown that the MEN-triggered release depended on 
both the field's magnitude and frequency. Particularly, we described a physical concept according to which the d.c. field component was used for the navigation purpose (through controlling spatial field gradients) while the a.c. field component was used for the controlled drug release off the MEN-based carrier (1). The physics of the a.c. triggered release was explained through the frequency-dependent spin-initiated torque. Indeed, our results confirmed that increasing the frequency reduced the disassociation field Figure 5. This trend was observed for all the three-functional layer types. The three materials allowed to significantly increase the magnitude and frequency control ranges.

To control the value of the dissociation field, we varied the effective binding strength between the MEN and the drug molecule $\left(F_{M D}\right)$. By introducing the intermediate (functionalization) layer between the MEN and the drug molecule, it is important to maintain the following inequality, $F_{M I}>F_{I D}$, where $F_{M I}$ and $F_{I D}$ stand for the binding forces between the MEN and the intermediate layer and between the intermediate layer and the drug molecules, respectively. The field-dependent photo-absorption measurements (Figure 5) indicated that the lowest and highest disassociation field strength and frequency values for GMO and EDC functionalization layers, respectively, with Tween-20 being in the middle. In fact, it should be mentioned that the full (100\%) release could be easily achieved for GMO (with field strengths of less than 100 Oe even at zero frequency), only $50 \%$ release could be achieved for Tween-20 and EDC with the field strength and frequency ranges available in the current study (due to the equipment limitations). In other words, a substantially higher threshold magnetic field (>200 Oe at zero frequency) is required to release over $50 \%$ of the PTX drug from either Tween-20- 
MENs or EDC-MENs. This observation correlates with the Zeta-potential measurements that show the deviation of the Zeta potential for the functionalized MENs from the Zeta potential of the non-functionalized MENs. The Zeta potentials values for Tween-20 and EDC-coated MENs, $-34.9 \pm 0.20 \mathrm{mV}$ and $-30.4 \pm 0.81 \mathrm{mV}$, respectively, are higher compared to that for the non-functionalized MENs, $-45.0 \pm 1.72 \mathrm{mV}$, and GMO-coated MENs, $-41.6 \pm 0.26 \mathrm{mV}$. The two latter combinations require only 10 and 100 Oe fields to release approximately $70 \%$ and $97 \%$ of PTX, respectively.

One side effect of the surface functionalization is the effect on the drug loading percentage and drug-binding efficacy (160-162). It is important to consider and if possible take advantage of this effect when tailoring the properties to control the dissociation field. This effect is reflected in our results (Figure 3). For example, the PTX loading amount was increased by over 75 and $80 \%$ after MENs were functionalized with Tween-20 and EDC, respectively, for a 3-hour incubation period, compared to that for the non-functionalized MENs. These results were also consistent with the circulatory system binding efficacy results, where EDC displayed over 91\% drug intactness to the nanoparticles (EDC-MENs) after circulating for 2 hours at $8 \mathrm{ml} /$ minute, which is the human coronary flow rate per minute per gram of the myocardium (163). Finally, the invitro cytotoxic experiment (Figure 39) indicated that neither of the nanoparticle types (MENs, GMO-MENs, of Tween-20-MENs) displayed any detectable toxicity, as long as the nanoparticle concentration was kept below $50 \mu \mathrm{g} / \mathrm{ml}$. Moreover, GMO-MENs displayed no toxicity at all ( $\sim 100 \%$ cell viability) even at the highest concentration values under study (above $100 \mu \mathrm{g} / \mathrm{ml}$ ), indicating the true biocompatible nature of the GMO 
$(164,165)$. The EDC-MENs showed a slightly higher toxicity, i.e. only $77 \%$ cells were viable after 48 -hour treatment at $50 \mu \mathrm{g} / \mathrm{ml}$ concentration. By reducing the concentration of EDC-MENs to $20 \mu \mathrm{g} / \mathrm{ml}$ we could increase the cell viability to over $90 \%$. (Note: AFM analysis, Mass spectroscopy analysis and XRD analysis of the drug release process is presented in the supplementary file)

\subsection{Conclusions}

This study demonstrated the unique ability of the recently developed body-temperature MENs-coated with intermediate (functionalization) layers, such as GMO, Tween-20, and EDC to control the drug release threshold field in wide ranges both in magnitude and frequency domains. While relatively small fields $(\sim 10$ Oe $)$ might be enough for navigating the drug in superficial body layers, relatively higher fields $(>100 \mathrm{Oe})$ are required for navigating the nanoparticles deep in the body. 30-nm $\mathrm{CoFe}_{2} \mathrm{O}_{4}-\mathrm{BaTiO}_{3}$ coreshell structures were used, as the MEN carriers. Using the described functionalization layers could also increase the bioavailability of the drug, as they provide higher binding efficacy and thus result in minimal drug loss before it reaches the target. Because of the quantum-mechanically caused high-energy efficiency of the MENdriven delivery, MENs could also carry higher drug payloads compared to the conventional MNs and release almost $100 \%$ of the drug on demand (via application of a low-energy a.c. field) to the target tissue. Using a popular anti-cancer drug, Paclitaxel, we demonstrated how to control the dissociation field for releasing drug from the MEN carrier by choosing the right functionalization layer. Through a comprehensive set of 
experiments including FTIR, photo-absorption, XRD, AFM, zeta-potential measurements, blood-circulation environment simulations, and in-vitro studies on SKOV-3 cell lines, a release of the drug on-demand was achieved at the following three different sub-ranges, (i) low (<10 Oe), (ii) moderate (100 Oe), and (iii) high ( $>200 \mathrm{Oe})$, respectively, by selecting the three functionalization layers, GMO, Tween-20, and EDC, respectively. Finally, the toxicity of the different MEN-functionalization layer combinations was studied on SKOV-3 cell lines using XTT assay at the particle concentration of $50 \mu \mathrm{g} / \mathrm{ml}$, which resulted in the following percentages of viable cells: (i) non-functionalized MENs: 96\%, (ii) GMO-MENs: 100\%, (iii) Tween-20-MENs: $89 \%$, (iii) EDC-MENs: 77\%. In summary, these results suggest that the functionalization of MENs paves a way for a substantially broader application of the new field-controlled nanocarriers (MENs) in targeted drug delivery.

Note: How to cite this chapter: The content of this chapter is published in my Particle \& Particle System Characterization article, hence please use this as a reference: Rakesh Guduru, Sakhrat Khizroev. "Magnetic Field-controlled Release of Paclitaxel Drug from Functionalized Magneto-Electric Nanoparticles” Particle \& Particle System (2013) 
Chapter 5:Magneto-electric Nanoparticles for Non-invasive Brain Stimulation 


\subsection{Introduction}

The signaling in a biological neural network is based on a highly collective system of electric charges, neurotransmitters and action potentials. The ability to incite the neuronal charge excitations from outside with the purpose to artificially stimulate the neural network remotely (non-invasively) remains an important roadblock to enable leapfrog advances in the important area of neuroscience and related applications in medicine and neural engineering. A neural network can be considered, as a complex electric circuit made of many neurons connected through synapses formed between axons and dendrites. Both types are known, as chemical and electrical synapses, respectively, transfer information between adjacent axons and dendrites directly or indirectly through electric field energy. Consequently, the neural network is sensitive to external electric fields. Moreover, the ability to efficiently control the network at micro- or even nano-scale can enable unprecedented control of important brain functions. The underlying physics is still an open question because of the many technical difficulties associated with direct studies of the brain functions. The existing technology typically relies on invasive direct-contact electrode techniques, such as Deep Brain Stimulation (DBS), which is one of only a few neurosurgical methods allowed for blinded studies. Existing non-invasive brain stimulation methods include repetitive transcranial magnetic stimulation (rTMS) (166, 167) and transcranial direct current stimulation (tDCS) $(168,169)$. rTMS and tDCS represent major advances of the state of the art in noninvasive brain stimulation, but the depth and locality focusing are limited in both methods (170). In rTMS, high intensity magnetic fields are required to stimulate deep brain regions but high intensity magnetic fields may lead to undesirable side effects (171). One potential solution to overcome the 
important roadblock for enabling non-invasive control of the neural network is to exploit the new concept of using magneto-electric (ME) nanoparticles we propose. ME materials represent a sub-group of multiferroic materials that are of great interest to the research community because of their ability to couple magnetic and electric fields at room temperature $(172,173)$. To our knowledge, our study for the first time describes the application of ME nanoparticles to the area of brain stimulation. Particularly, our approach relies on using ME nanoparticles to achieve the following important features for noninvasive monitoring and stimulation the brain activities. First, using ME material nanoparticles through temporarily injected aqueous solutions is the key enabler for efficient coupling between magnetic and electric fields at nano- and/or micro-scale over the entire brain volume. Particularly, remotely controlled magnetic fields, instead of electric fields, can be used to induce strong local electric charge oscillations (in ME nanoparticles) that can directly interact with the neural network and therefore be used for localized and targeted brain stimulation (Refer Figure 40). Unlike surface-limited electric fields that are typically generated by invasive contact electrodes, magnetic fields generated by injected ME nanoparticles can effectively penetrate the entire brain noninvasively, and be "switched" on and off remotely using external low-energy magnetic field sources. The nanoparticles must satisfy certain requirements on the strength of magneto-electric (ME) coupling (defined by the ME coefficient). Second, magneto-electric nanoparticles must be smaller than approximately $20 \mathrm{~nm}$ in diameter to penetrate the blood-brain barrier (BBB). Having the size of the ME nanoparticles within the BBB-defined boundary enables adequate delivery of the nanoparticles into selected brain regions. (There are many chemical and physical processes to synthesize ME 
nanoparticles with the required parameters. For instance, ion beam proximity lithography (IBPL) is a physical method that can be used to fabricate nanoparticles with a wide range of sizes, ranging from sub-10-nm to over $50 \mathrm{~nm}$ in diameter $(174,175)$. Third, only very low intensity external magnetic field is required to stimulate brain activity at any depth in the brain. The external magnetic field can be focused to act upon ME nanoparticles in any particular region of the brain. The external magnetic field generates AC signals in ME nanoparticles that are correlated with the frequency spectrum of the neural charge activity, which in turn causes neurons in the region to fire at similar frequencies (Figure 40). For example, provided an adequately large ME coefficient, low-energy magnetic coils can be used to trigger the required stimulation, as described below in more detail.

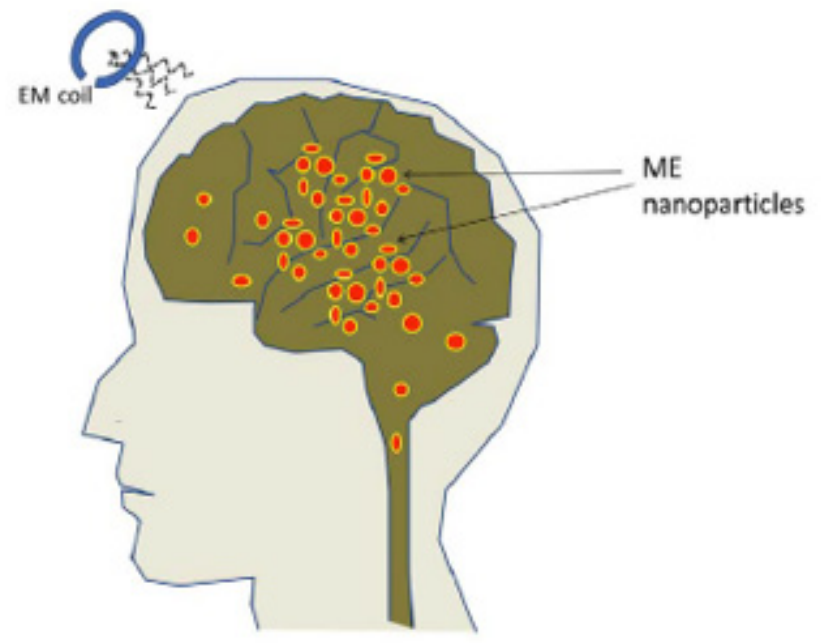

Figure 40: Illustration of MENs technology for deep brain stimulation 


\subsection{Experimental methods}

\subsubsection{Simulation}

Below we describe the foundation of the modeling approach we used to model the effects of MENs (with their externally excited electric and magnetic moments) on the brain activity. The computational procedure was built on top of a conventional model used to simulate the electric field dynamics in the neural network. For example, see the article by So et al to understand the underlying principles of the conventional model (176). To account for the effect of ME nanoparticles, we made the following assumptions that can be justified at this early stage of research. First, with the average diameter of the ME nanoparticles below $20 \mathrm{~nm}$ in a neural system with the smallest feature size of at least an order of magnitude higher, we can use a trivial point-dipole approximation to model the electric field by each local nanoparticle (177). Second, we assume a uniform distribution of nanoparticles in a brain region under study. The assumption is valid because the nanoparticles, due to their sub-20-nm average diameters, experience a relatively negligible resistance from the surrounding tissues and therefore their spatial distribution in the ground state can be controlled by an external magnetic field. The electric dipole moment of each nanoparticle, $\mathrm{P}$, is determined by the external magnetic field, $\mathrm{H}$, according to the expression, $P_{i}=\sum_{j} \alpha_{i j} H_{j}$, where $\alpha_{i j}$ is the $1^{\text {st }}$ order linear magnetoelectric (ME) tensor coefficient. Therefore, assuming an isotropic matrix (with identical diagonal coefficients and zero non-diagonal coefficients) with the typical value for $\alpha_{i j}$ of

$100 \mathrm{~V} \mathrm{~cm}^{-1} \mathrm{Oe}^{-1}$ and a local magnetic field of $300 \mathrm{Oe}$, the polarized moment at the location of the nanoparticle would be $30 \mathrm{kV} \mathrm{cm}^{-1}$. In the current arrangement, the purpose 
of MENs is to act, as additional deep brain sources of electric fields that can be precisely controlled by external magnetic fields because of the non-zero ME constant. The nanoparticles can be considered, as finely controlled deep brain local stimulation switches that can enable high-precision (with nanoscale localization) and high-throughput (energy-efficient) noninvasive medical procedures. To artificially trigger (stimulate) electric pulses in the brain region under study with the purpose to prevent or compensate for any illness-caused malfunctions or lapses in a periodic chain of electric signals in the parts of neural system occupied by the nanoparticles, we would apply a.c. magnetic fields at the matching frequencies, as described below in more detail.

\subsection{Results}

In this study, we computed the effects of MENs at different concentrations and matching frequencies to stimulate four different regions of the brain (healthy and Parkinson's): The four regions are: i) Thalamic, ii) Sub-thalamic (STN), iii) Globus pallidus external (GPe), and iv) Globus pallidus internal (GPi). The computed typical periodic pulses in four region of the healthy person's brain are presented in Figure 41. It should be noted that the healthy person's brain signals are very periodic and uniform in amplitude whereas, the signals in the individual with the Parkinson's disease are more random and discrete (Figure 42). Once optimized the simulation parameters, the results in four regions of the Parkinson's diseased brain using MENs procedure and using DBS procedure are shown in Figure 43 and Figure 44 respectively. 


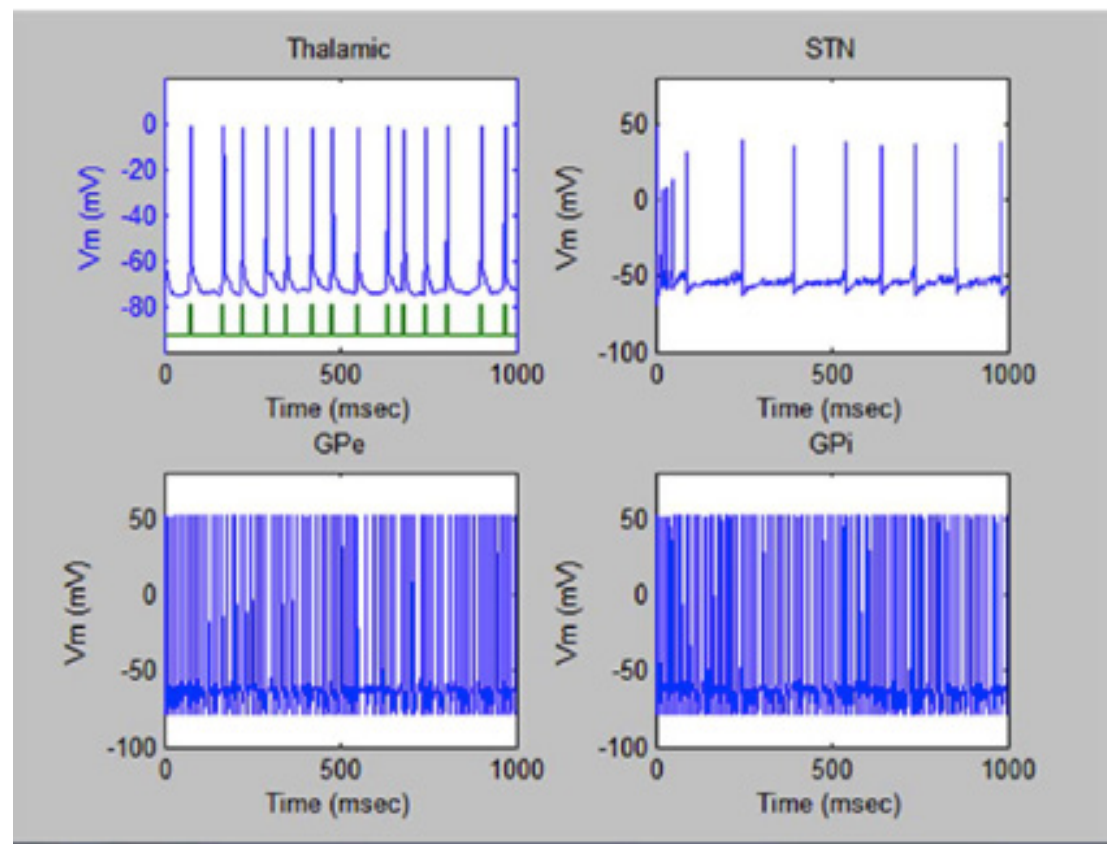

Figure 41: Typical electrical activity in four regions (thalamic, sub-thalamic, globus pallidus-external, and globus-internal) of the brain in a healthy individual.

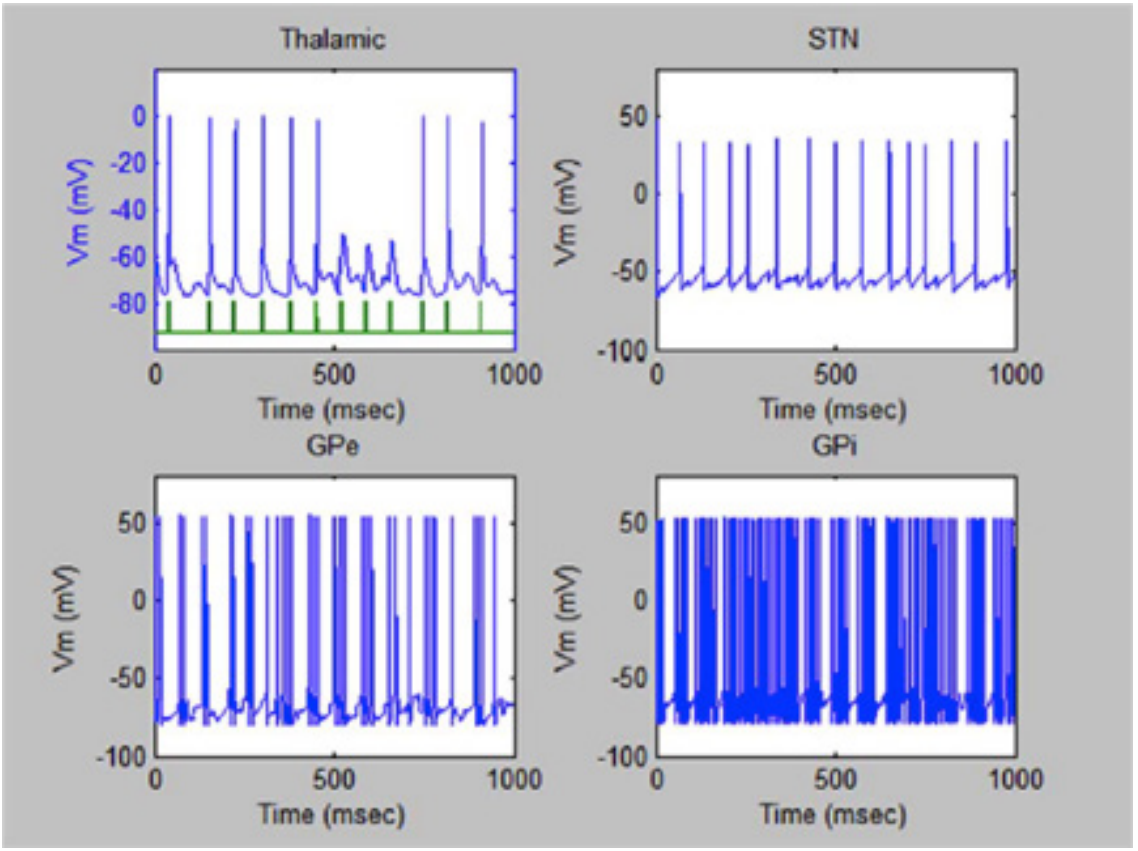

Figure 42: Discrete electrical activity in four regions (thalamic, sub-thalamic, globus pallidus-external, and globus-internal) of the brain in an individual suffering from Parkinson's. 


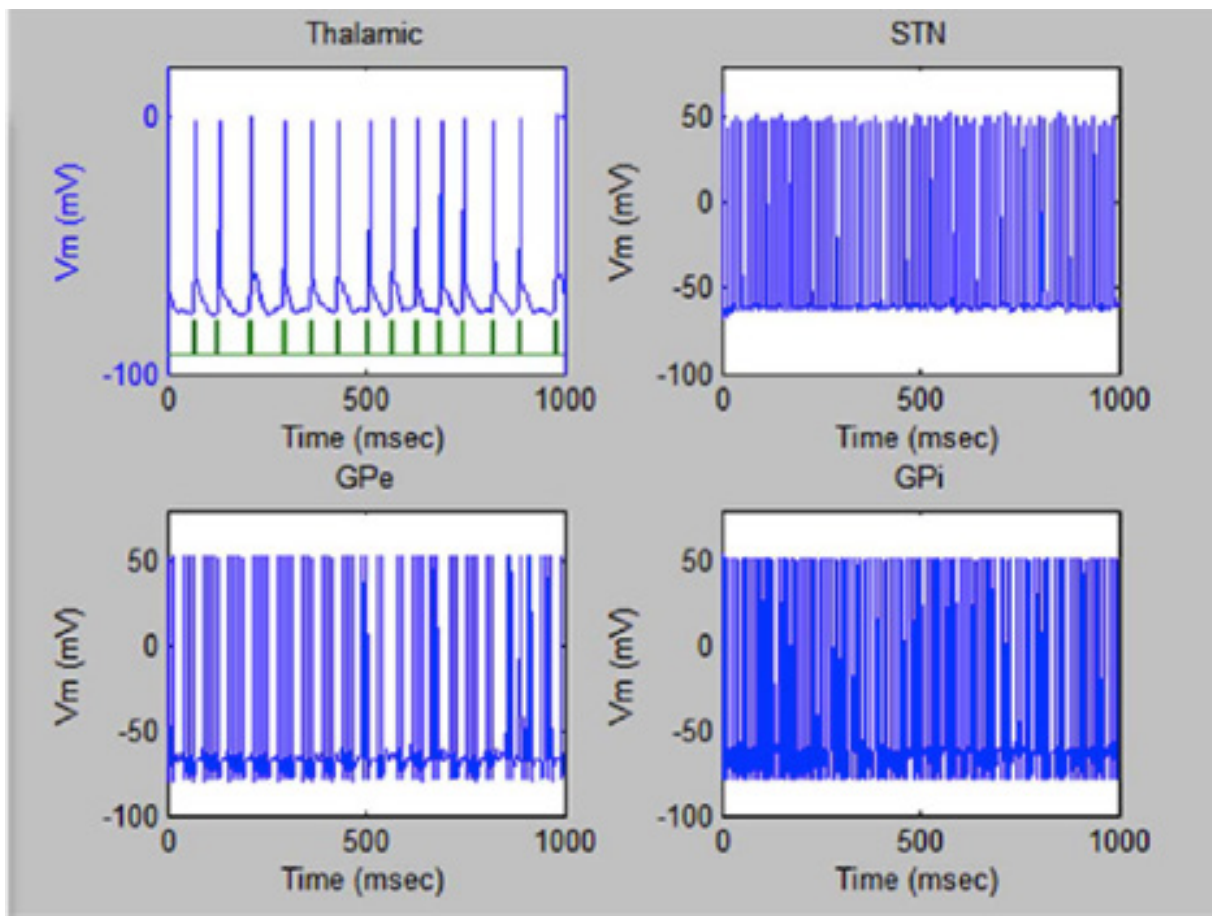

Figure 43: Restored-electrical activity in four regions (thalamic, sub-thalamic, globus pallidus-external, and globus-internal) of a brain in an individual suffering from Parkinson's after treating with MENs non-invasively.

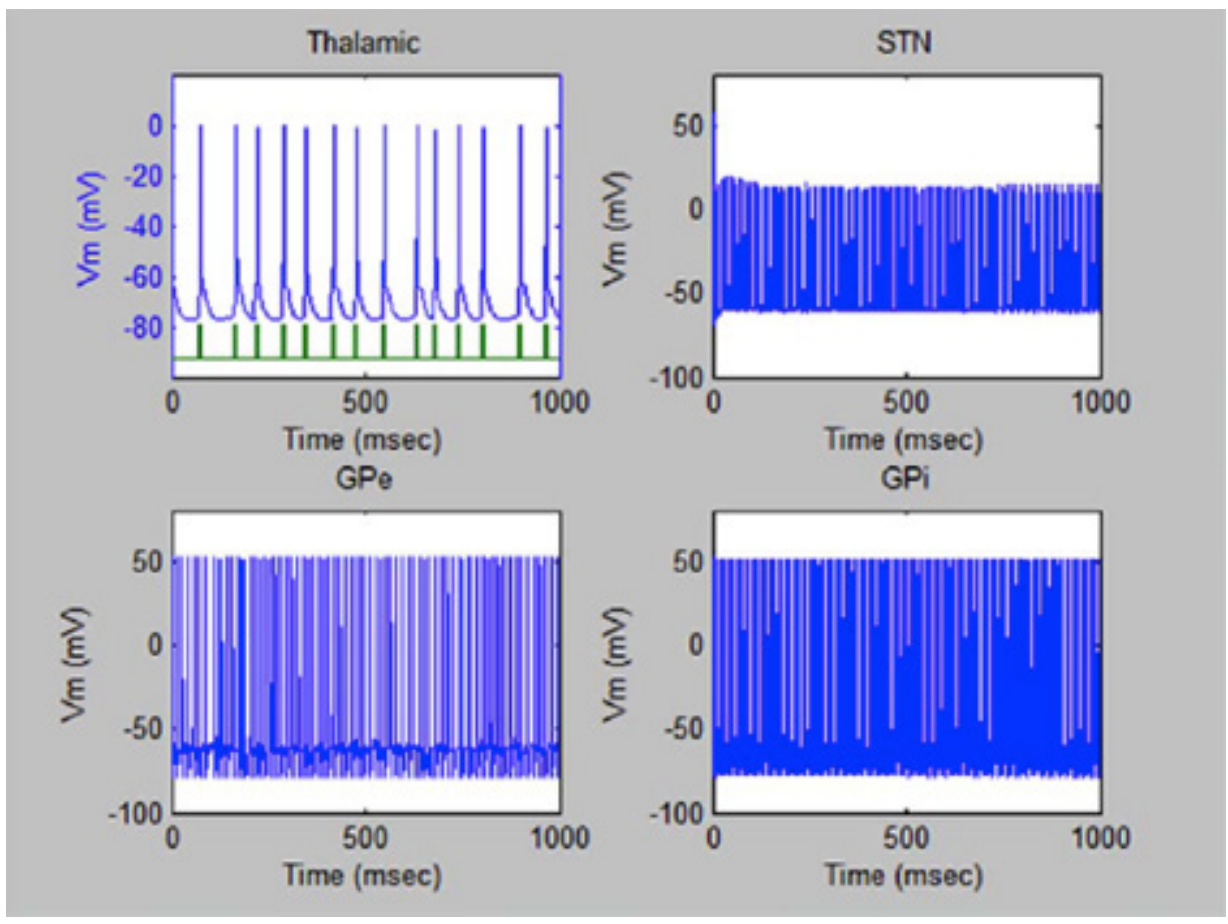

Figure 44: Restored-electrical activity in four regions (thalamic, sub-thalamic, globus pallidus-external, and globus-internal) of a brain in an individual suffering from Parkinson's after treating with invasive DBS treatment. 


\subsection{Discussion}

We studied the effects (on the potential recovery of the deteriorated electric field pulses) of the injected MENs (in solutions) at different concentrations and stimulation frequencies (generated by a 300-Oe external a.c. magnetic field source). The field amplitude of 300 Oe was chosen from the requirement to maintain an energy-efficient operation while keep MENs saturated during the procedure. The aqueous solution concentration of the nanoparticles was varied from 0 to over $10^{7}$ particles/cc while the frequency was varied in the range of interest, i.e. from 0 to over $1 \mathrm{kHz}$. Within the ranges of the modeling parameters, the optimum values for the nanoparticle concentration and the magnetic field excitation frequency were found to be $3 \times 10^{6}$ particles/cc and $80 \mathrm{~Hz}$, respectively. Figure 41 illustrates typical periodic pulsed time sequences generated in the four parts of the brain of a healthy person under normal conditions. It can be noted that all the electric field pulses are quite periodic and uniform in amplitude. No lapses in the periodic sequence can be detected. For comparison, Figure 42 illustrates typical signals in the same four parts of the brain of the patient suffering from Parkinson's disease. The most drastic difference from the case of the healthy person is an appearance of pronounced lapses in the periodic pulsed sequences particularly in the thalamic region. Also, the periodicity of the pulses in the other regions is broken. Figure 43 illustrates the four-pulsed sequences under study, as a result of the procedures with the optimized stimulation parameters. It can be observed that the most dramatically damaged signals in the thalamic region were fully recovered during the procedure. At least partial recovery of the periodicity in the other three regions can also be observed. For comparison, the ME nanoparticle stimulation still outperformed the invasive DBS procedure with electric 
signal stimulation. The "recovered" signals in the four regions of the brain during the DBS procedure are shown in Figure 44. In this case, not only the periodicity was not fully recovered, but also the amplitude of the signal in the STN region deteriorated compared to the normal operation.

\subsection{Conclusions}

In conclusion, we modeled the effect of magneto-electric nanoparticles to non-invasively stimulate the brain of a patient with Parkinson's disease. Using the optimized values for the concentration of the 20-nm nanoparticles (with the magneto- electric (ME) coefficient of $100 \mathrm{~V} \mathrm{~cm}^{-1} \mathrm{Oe}^{-1}$ in the aqueous solution) of $3 \times 10^{6}$ particles/cc and excitation frequency of the externally applied 300-Oe magnetic field of $80 \mathrm{~Hz}$, the pulsed sequences of the electric field were brought to the levels comparable to those of healthy people. The preliminary results of this study suggests that using ME nanoparticles can lead the way to implementing nanotechnology to improve our understanding of the biological neural network and develop new nano-medical methods for non-invasive monitoring, preventing, and treatment of brain and other neural system diseases.

Note: How to cite this chapter: The content of this chapter is published in my PLoS one article, hence please use this as a reference: Yue, Kun, Rakesh Guduru, Jeongmin Hong, Ping Liang, Madhavan Nair, and Sakhrat Khizroev. "Magneto-electric nano-particles for non-invasive brain stimulation." PloS one 7, no. 9 (2012): e44040. 
Chapter 6: Brain Activity Mapping Using Magneto-electric Nanoparticles Noninvasively 


\subsection{Introduction}

Ability to map the neuronal activity in the brain non-invasively is one of the greatest scientific challenges of all time. Complete mapping of the neuronal activities in the brain and correlating them to the behavioral or functional responses could help us in better understanding of the brain's function in different mental and behavioral states. Such an understanding is the key to accurately diagnose and treat several neurological and psychological disorders. Although, over the last two decades there have been numerous attempts to accomplish the same using the invasive and noninvasive methods, the complete theory of the brain's function is poorly understood till date (178). Several drawbacks of these current methods are discussed in detail in the previous section (1.6). One potential solution to overcome these drawbacks for non-invasive mapping of the neuronal activity in the brain is to exploit the use of magneto-electric property of MENs (Nobel properties of the MENs are presented in the section 1.3.3). To our knowledge, the use of MENs for monitoring the neuronal activity in the brain was never studied before. To accomplish this, MENs (in aqueous solution) need to be intravenously injected temporarily into the subject's body. Using external magnetic fields these particles can be distributed over the entire volume of the brain $(39,179)$. Once these particles are distributed, the surrounding electrical conductivity of the brain tissue can influence the surface charge of the MENs, which further affects the magnetic moment of the particles (1). This change in magnetic moment can be recorded using MRI and MNI imaging modalities (180), thus providing the electric field/conductive map over the entire brain volume. This property of the MENs can also be exploited to track the neuronal activity of the brain. For example, when neurons are fired, the surface charge distribution of the 
neuronal membrane will change, as a result of propagation of action potential (181), thus affecting the magnetic moment of the MENs in their vicinity. Figure 45 shows the change in the magnetic moment of the MENs (black to orange) in the vicinity of the neurons where action potential (blue) is generated. Dependence of magnetic moment of the MENs on surface charge is described in our previous publication (1).

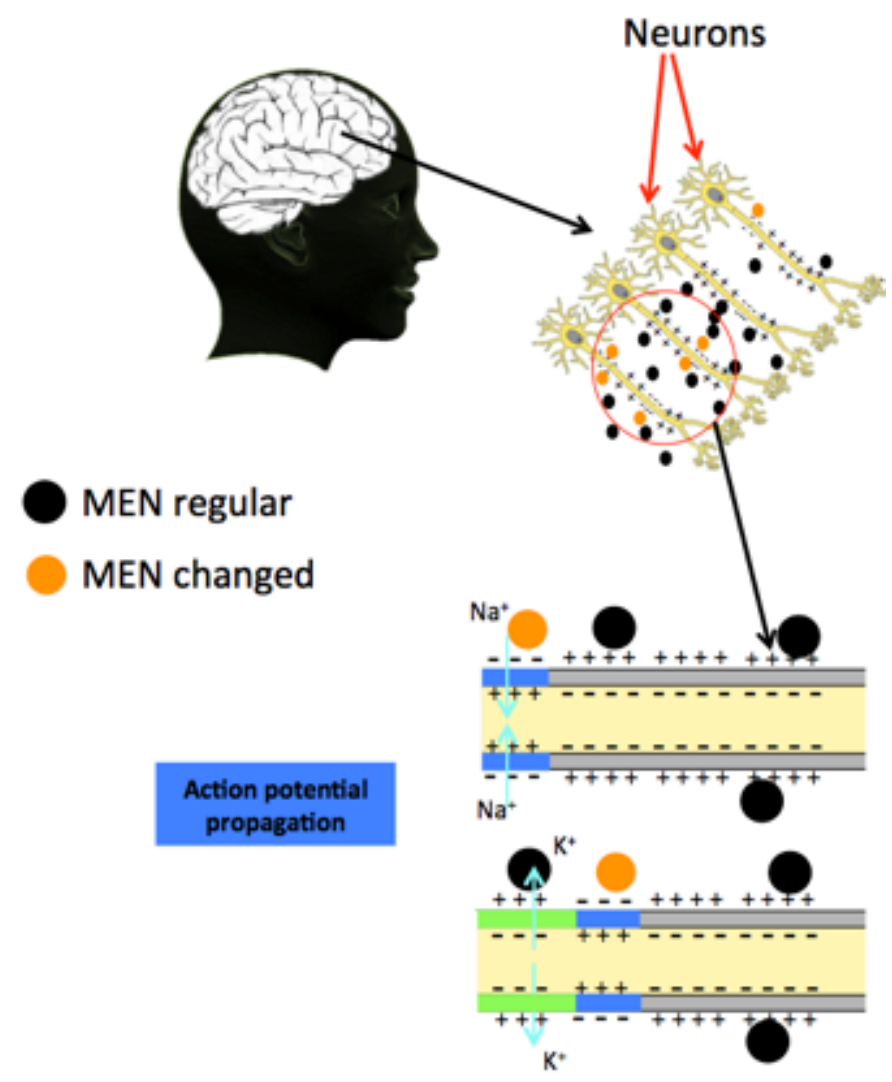

Figure 45: Artistic view of the MENs interaction with neurons. The surface property of the MENs changes under the influence of surroundings microenvironment thereby changing their magnetic moment. 
In this study, mathematical simulation results of MRI and MNI contrast dependence on the surface charge, size, and concentration of the MENs are presented. (Note: for comparison, contrast dependence of regular magnetic particles (MNs) is also presented). Of all properties (i.e. charge, size, and concentration), our results indicated that the only property that is influenced by the environmental conditions is the surface charge of the MENs. Hence, we exploited this dependence to map the electrical activities of the brain. The results showed a strong MRI signal in different regions of the brain based on the type of activity it performs.

\subsection{Experimental methods}

\subsubsection{Simulation of relative magnetization of nanoparticles}

Field dependent relative magnetization of MENs and MNs can be simulated using LANGEVIN function (L) shown in Equation 3 (182) where $\alpha$ is the relative magnetization $\left(\mathrm{M}_{\mathrm{rel}}\right)$. Equation 4 and Equation 7 were used to simulate the $\mathrm{M}_{\mathrm{rel}}$ for MNs and MENs respectively using a single particle model to study the effect of surface charge and size (183). Equation 5 and Equation 8 were used to simulate the $\mathrm{M}_{\mathrm{MMF}}$ for MNs and MENs respectively by expanding the single particle model to the bulk concentrations, i.e. from the second order modified mean-field theory to study the effect of concentration of the particle (183).

$$
L(\alpha)=\operatorname{coth}(\alpha)-\frac{1}{\alpha}
$$




\section{Magnetic nanoparticles:}

$\operatorname{Mrel}, S P M(H)=\rho M s\left\langle L\left(\frac{\mu o M s \frac{\pi}{6} d^{3} H}{K b T}\right)\right\rangle$

$M_{M M F}(H, \rho)=\rho M_{S}\left\langle L\left(\frac{\mu o M s \frac{\pi}{6} d^{3} H_{e}}{K b T}\right)\right\rangle$

Where,

$H_{e}=H+\frac{1}{3} \rho M_{S}\left\langle L\left(\frac{\mu o M s_{6}^{\frac{\pi}{6}} d^{3} H}{K b T}\right)\right\rangle \cdot\left(1+\frac{1}{48} \rho M_{S} \frac{d}{d H}\left\langle L\left(\frac{\mu o M s_{\frac{6}{6}} d^{3} H_{e}}{K b T}\right)\right\rangle\right.$

Magneto-electric nanoparticles:

$\operatorname{Mrel}, \operatorname{SPM}(H)=\left\langle L\left(\frac{\mu o \alpha \frac{Q}{\pi d^{2}} H}{K b T}\right)\right\rangle$

$M_{M M F}(H, \rho)=\rho M_{S}\left\langle L\left(\frac{\mu o \alpha\left(\frac{Q}{\pi d^{2}}\right) H_{e}}{K b T}\right)\right\rangle$

Where,

$H_{e}=H+\frac{1}{3} \rho M_{S}\left\langle L\left(\frac{\mu \circ \alpha\left(\frac{Q}{\pi d^{2}}\right) H}{K b T}\right)\right\rangle \cdot\left(1+\frac{1}{48} \rho M_{S} \frac{d}{d H}\left\langle L\left(\frac{\mu \circ \alpha\left(\frac{Q}{\pi d^{2}}\right) H}{K b T}\right)\right\rangle\right.$

Signal simulation for surface charge effect of the nanoparticle: To study the effect of the surface charge of the nanoparticle on MRI/MNI signal intensity, the following parameters were considered: nanoparticle size as $50 \mathrm{~nm}$ (for MEN and $\mathrm{MN}$ ), magnetic saturation as $480000 \mathrm{~A} / \mathrm{m}$ for magnetite, Magneto-electric coefficient $(\alpha)$ as $10,000 \mathrm{vm}^{-1} \mathrm{Oe}^{-1}$ (for MEN), Boltzmann constant $(\mathrm{Kb})$ as $1.38 \times 10^{-23}$, temperature as $300 \mathrm{~K}$, permeability $(\mu o)$ as $4 \pi \times 10^{-7} \mathrm{H} / \mathrm{m}$ and surface charge as $\mathrm{Q} 1=0.25 \times 10^{-35} \mathrm{C}, \mathrm{Q} 2=0.5 \times 10^{-35} \mathrm{C}, \mathrm{Q} 3=1 \times$ $10^{-35} \mathrm{C}, \mathrm{Q} 4=2 \times 10^{-35} \mathrm{C}, \mathrm{Q} 5=5 \times 10^{-35} \mathrm{C}$ (For MEN). Note: charge is not considered for the $\mathrm{MN}$ as the relative magnetization is not affected by its charge on the surface. 
Signal simulation for the size effect of the nanoparticle: To study the effect of the size of the nanoparticle on the MRI/MNI signal, the parameters were same, as above with the change of size of the nanoparticles from $20 \mathrm{~nm}$ to $100 \mathrm{~nm}$ (for both MEN and MN) and the surface charge for MEN was kept constant $\left(0.25 \times 10^{-35} \mathrm{C}\right)$.

Signal simulation for the concentration effect of the nanoparticles: To study the effect of the concentration of the nanoparticles on the MRI/MNI signal, the concentration of the nanoparticles was varied from $0.05 \mathrm{~mol} / \mathrm{lit}$ to $2 \mathrm{~mol} / \mathrm{lit}$. (i.e. $\mathrm{C} 1=0.05, \mathrm{C} 2=0.1, \mathrm{C} 3=$ $0.5, \mathrm{C} 4=2 \mathrm{~mol} / \mathrm{lit}$ ). The size and charge of the MENs were considered as $50 \mathrm{~nm}$ (same for MNs) and $0.25 \times 10^{-35} \mathrm{C}$, respectively.

\subsubsection{MRI and MNI signal intensity simulations}

The contrasting agent signal intensity in the MR imaging modality systems (i.e. signal equation for two-dimensional spoiled gradient echo, 2DSPGR and three-dimensional spoiled gradient echo, 3DSPGR) was generated based on the Equation 5 (180).

$$
S_{M R I}=M \frac{\sin (F A)\left(1-\exp \left(-\frac{T R}{T 1}\right)\right)}{1-\cos (F A) \exp \left(-\frac{T R}{T 1}\right)} \times \exp \left(-\frac{T E}{T 2 *}\right)
$$

Where, $\mathrm{S}_{\mathrm{MRI}}=\mathrm{MRI}$ signal, $\mathrm{M}$ is the magnetization of the contrasting agents, FA is the flip angle, TR is the repetition time, T1 is the longitudinal relaxation time and $\mathrm{T} 2 *$ : is the transverse relaxation time. For the particular concentration of the contrasting agents, instrumental parameters, and the tissue types, the MRI signal S depends only on the magnetization of the contrasting agents $\left(S_{M R I} \alpha M\right)$. In case of MNI, the signal intensity is only dependent on the magnetization of the contrasting agents and independent of instrumental parameters and tissue type $\left(S_{M N I}=M\right)$. Thus, in both MRI and MNI, magnetization of the contrasting agents plays a significant role in their signal intensities. 


\subsubsection{Brain segmentation of the MRI data}

The MRI head and the diffusion tensor image (DTI) data of the brain were obtained from the 3D-Sclicer open source software database (184). Both images (i.e. MRI and DTI) were co-registered using a Brainstorm, Matlab application software (185). The coregistered MRI data was segmented into white matter (WM), gray matter (GM), cerebrospinal fluid (CSF), skull, and scalp using Brainsuite (version 13a) image analysis software (185).

\subsubsection{Tissue properties}

All the segmented brain tissues were assumed to be isotropic (186), but the isotropic conductivity values were set only for WM $(0.14 \mathrm{~S} / \mathrm{m}), \mathrm{GM}(0.33 \mathrm{~S} / \mathrm{M})$, and CSF $(1.79$ $\mathrm{S} / \mathrm{m}$ ), as the nanoparticles in the scalp and skull region are absent at any given time point (187). The electrical field values for these conductive tissues were calculated using the

equation $E=\frac{J}{\sigma}$ where, $\mathrm{E}$ is the electrical field, $\sigma$ is the conductivity and $\mathrm{J}$ is the current density of the firing-neurons.

\subsubsection{Tracking the neuronal activity of the brain}

Intravenously injected aqueous solution of the MENs can be navigated to the brain and uniformly distributed over the entire volume using an external magnetic gradient system described elsewhere (41). Once the MENs are distributed over the entire brain, neuronal activity can be tracked using MRI or MNI imaging modalities. To simulate this idea, we segmented the MRI brain image based on the tissue type and the function it carries using Brainsuite 13a software. Depending on the type of function brain performs, the neurons 
in the associated tissues change their surface properties thereby changing the relative magnetization of the surrounding MENs.

\subsection{Results}

\subsubsection{MRI/MNI signal simulation}

In this study, we simulated the relative magnetization dependence on surface charge, size, and the concentration of the contrasting agents (MENs and MNs) under varying magnetic field (0 to $200 \mathrm{Oe})$.

\section{- The effect of surface charge of the nanoparticle}

MRI/MNI signal simulation results show that increasing the surface charge of a particle will increase the relative magnetization for magneto-electric nanoparticle (shown in Figure 46), as magnetic moment of the MEN depends on the surface charge (Equation 7). For magnetic nanoparticle, relative magnetization does not depend on the surface charge (from Equation 4), as shown in Figure 47. To show the effect of surface charge on the intensity of the MRI/MNI signal, relative magnetization at 50 Oe field was calculated from the chart shown in Figure 46 for MENs. Note: for comparison MRI/MNI signal for the MNs were also presented in Figure 47. These results indicate that increasing the surface charge of the MENs from $0.25 \times 10^{-35} \mathrm{C}$ to $5 \times 10^{-35} \mathrm{C}$ increased the MRI/MNI signal from $\sim 0.44$ to $\sim 0.96$, which is almost a $120 \%$ increase in the signal contrast. 


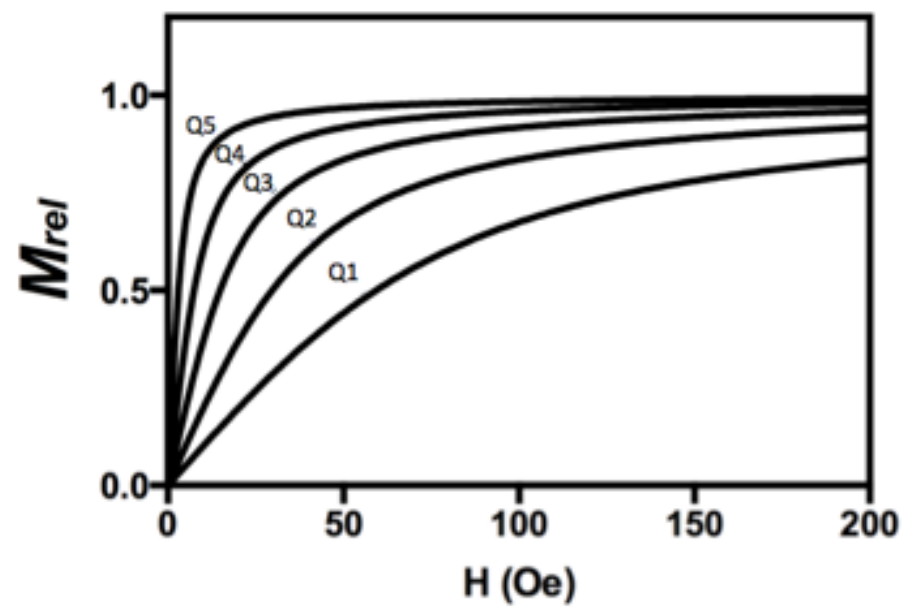

Figure 46: Relative magnetization curve showing the dependence on surface charge of the magneto-electric nanoparticles.

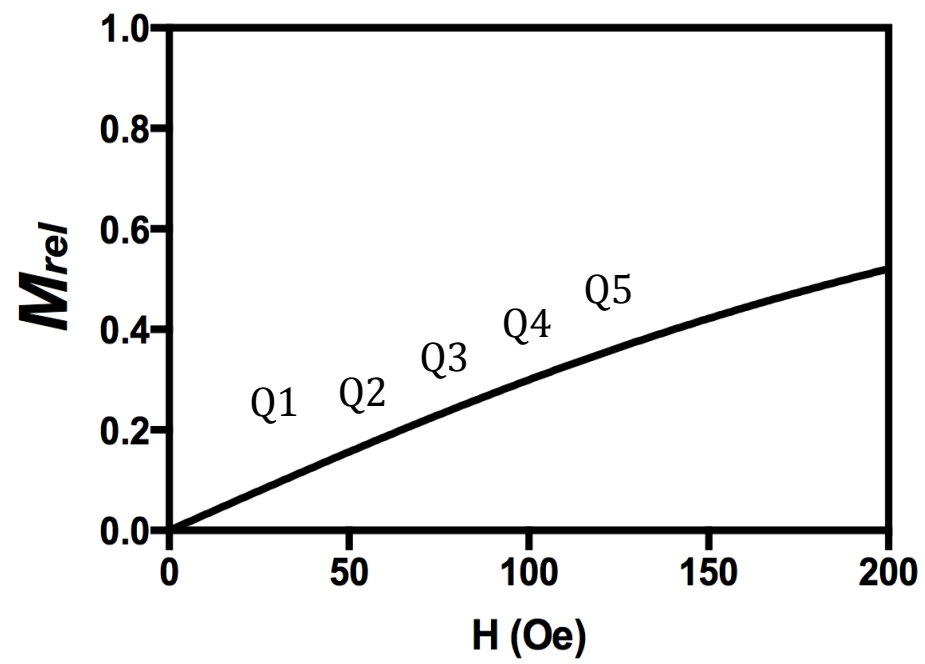

Figure 47: Relative magnetization curve for MNs. The chart shows no change in the magnetization curve for MNs having different surface charge $(Q 1, Q 2, Q 3, Q 4$, and Q5). 


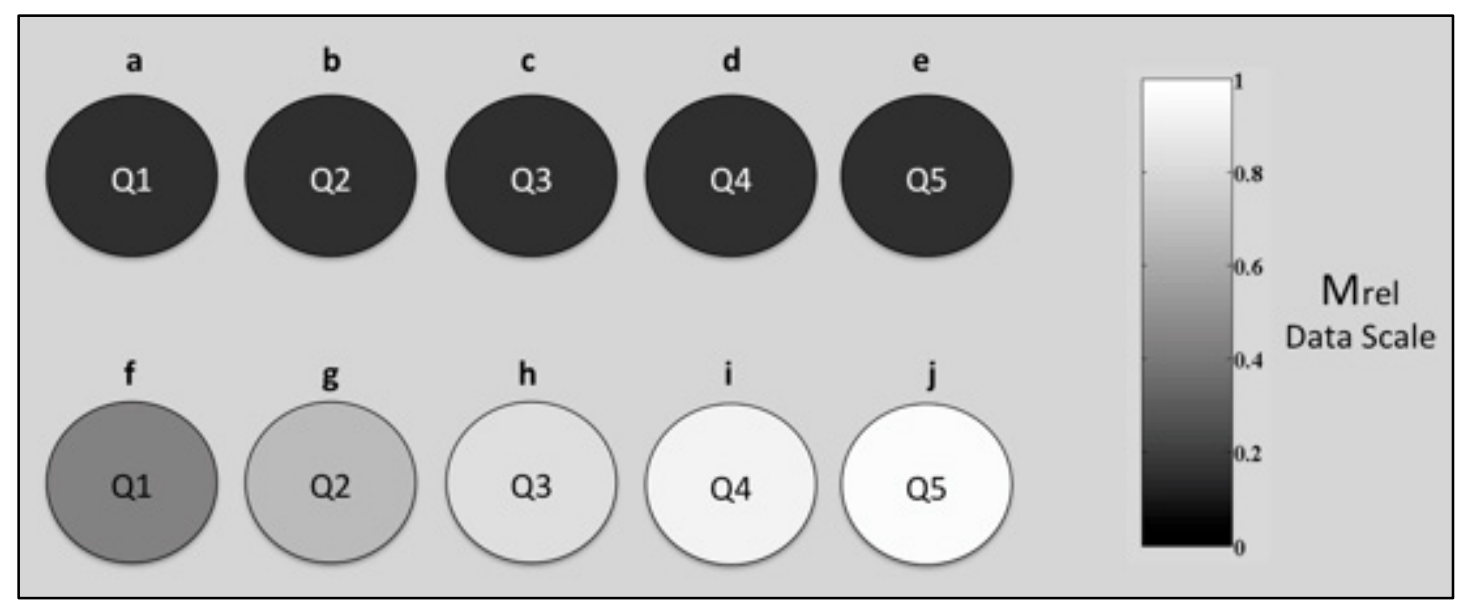

Figure 48: Showing the corresponding MRI/MNI signal for Relative magnetization (Mrel) change for different surface charge of the particle at $H=50$ Oe. Mrel for magnetite (a-e). Mrel for MENs (f-j).

- The effect of size of the nanoparticle

MRI/MNI single is also affected by the size of the nanoparticle (Equation 4 and 7). With the increase in the size of the MEN from $20 \mathrm{~nm}$ to $100 \mathrm{~nm}$, the relative magnetization of the nanoparticle significantly decreased (Figure 49), whereas increase in size of the MN decreases the relative magnetization (Figure 50). As a result, the MRI/MNI signal intensity decreases from $\sim 0.72$ (for $20 \mathrm{~nm}$ ) to $\sim 0.01$ (for $100 \mathrm{~nm}$ ) for MEN (e through $\mathrm{f}$ in Figure 51), and for $\mathrm{MN}$ it increases from 11.5 (for $20 \mathrm{~nm}$ ) to $~ 87.7$ (for $100 \mathrm{~nm}$ ) (a through d in Figure 51). 


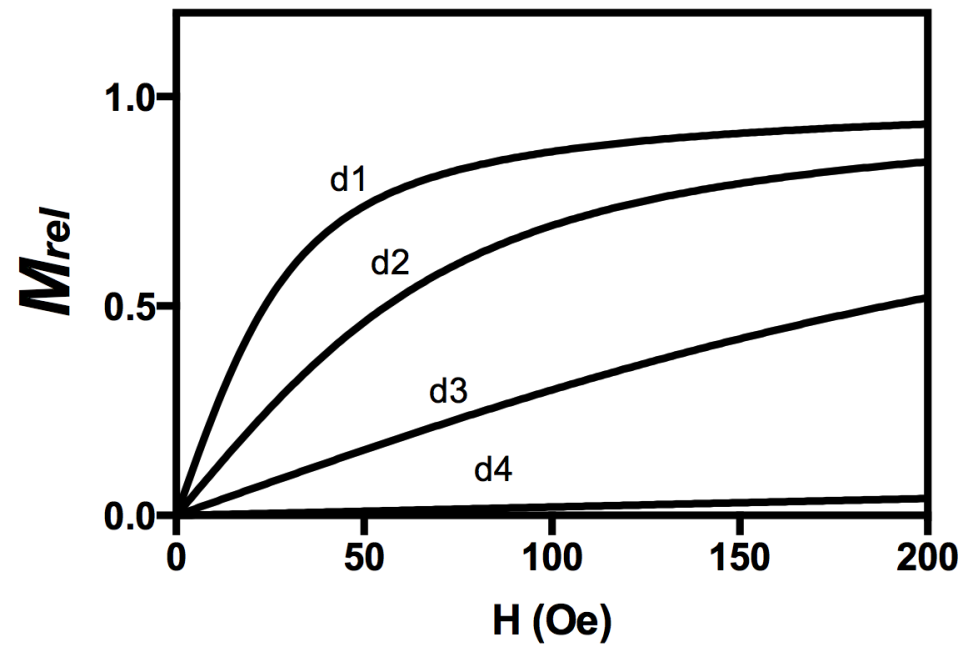

Figure 49: Relative Magnetization of the MEN for different particle sizes $(\mathrm{d} 1=\mathbf{2 0} \mathrm{nm}, \mathrm{d} 2=50 \mathrm{~nm}, \mathrm{~d} 3=\mathbf{7 5} \mathrm{nm}, \mathrm{d} 4=$ $100 \mathrm{~nm}$ ) keeping the charge $Q$ as constant. (Note: that Magnetization decreases when size is decreases, as $Q$ was kept constant. In reality surface charge depends on the size of the particle).

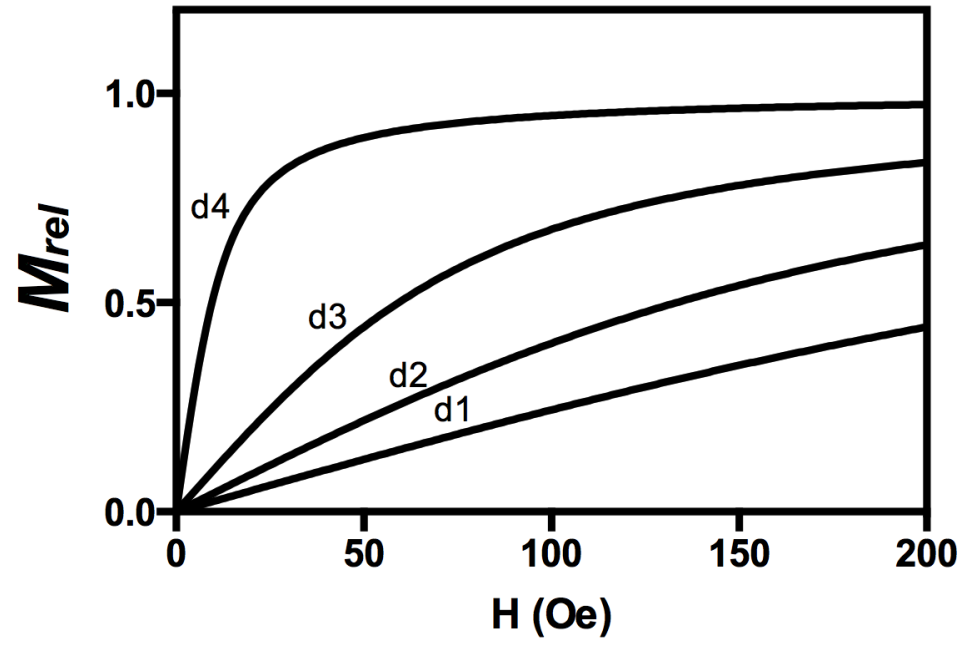

Figure 50: Relative Magnetization of the $M N$ for different particle sizes $(d 1=20 \mathrm{~nm}, d 2=50 \mathrm{~nm}, d 3=75 \mathrm{~nm}, d 4=$ $100 \mathrm{~nm})$. 


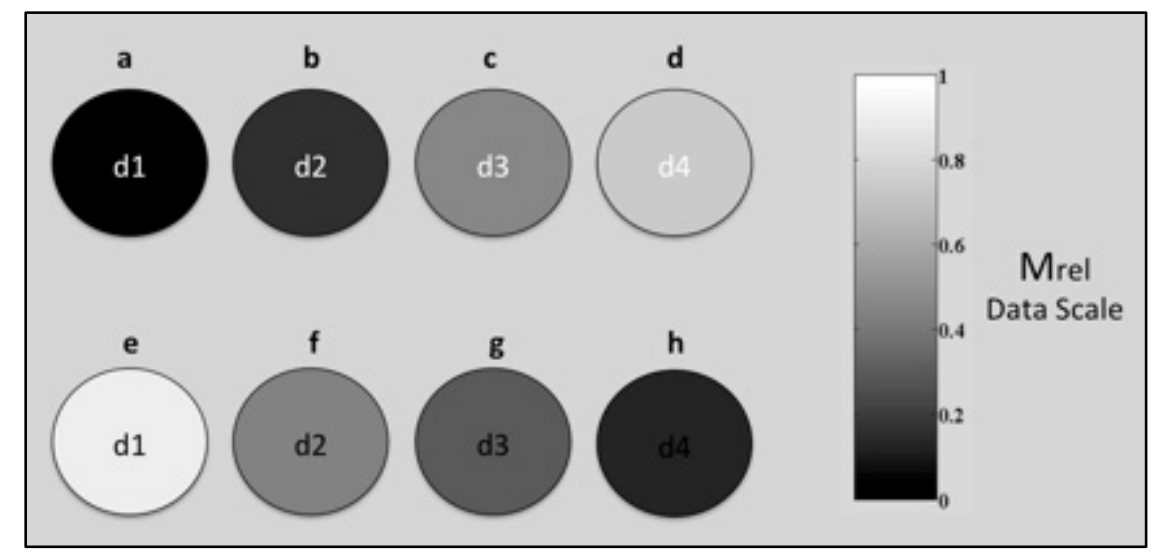

Figure 51: Showing the corresponding MRI/MPI signal for change in Relative magnetization (Mrel) at $\mathbf{H}=\mathbf{5 0}$ Oe for different size particles. Mrel for magnetite (a-d). Mrel for MENs (e-f).

- The effect of concentration of the nanoparticles

Increasing the concentration of the nanoparticles increases the relative magnetization of both the type of nanoparticles (MENs and MNs) from the Equation 5 and 8 (Figure 52 for MENs and Figure 53 for MNs). As a result, with the increase in the concentration of the MENs from 0.1 to $2 \mathrm{~mol} / \mathrm{lit}$, the MRI/MNI signal increased from $\sim 0.76$ to $\sim 0.98$ (a through d Figure 54) and for MNs the signal increased from $\sim 0.26$ to $\sim 0.98$ (e through $\mathrm{f}$ Figure 54). 


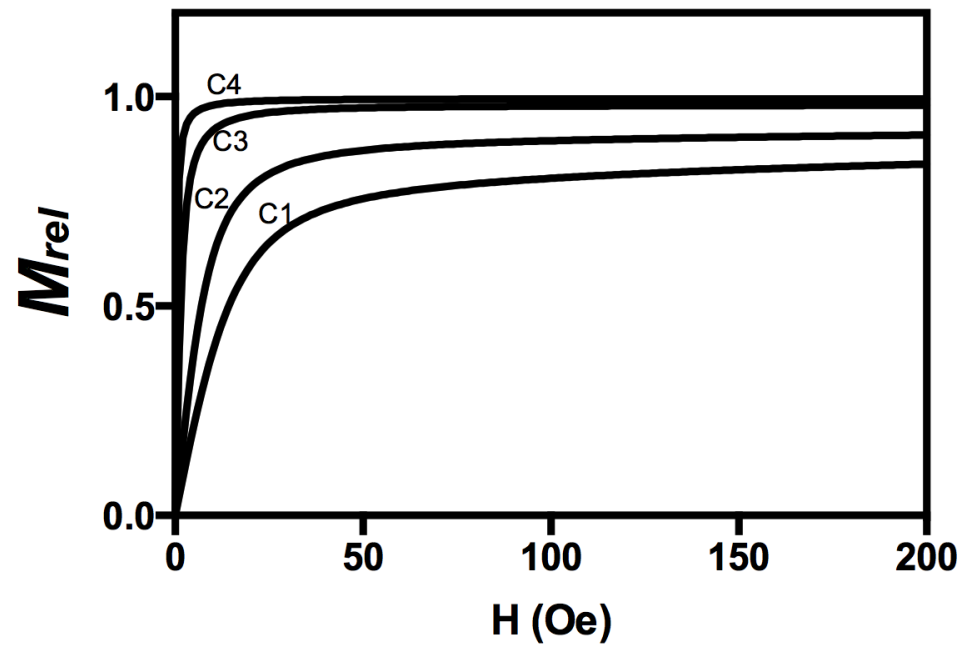

Figure 52: Relative Magnetization of Magnetoelctric nanoparticles at different concentration $(\mathrm{C} 1=0.05 \mathrm{~mol} / \mathrm{lit}$, C2 $=0.1 \mathrm{~mol} /$ lit, C3 $=0.5 \mathrm{~mol} / \mathrm{lit}, \mathrm{C} 4=2 \mathrm{~mol} / \mathrm{lit}$ ).

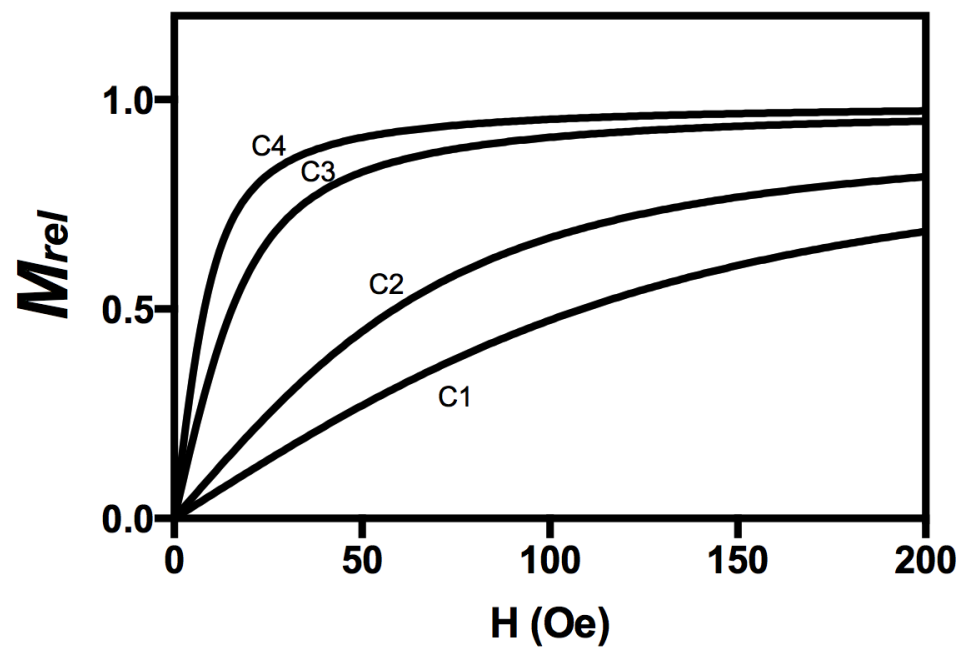

Figure 53: Relative Magnetization of Magnetic Particle (Magnetite) at different concentration $(\mathrm{C1}=0.05 \mathrm{~mol} / \mathrm{lit}$, C2 $=0.1 \mathrm{~mol} / \mathrm{lit}, \mathrm{C} 3=0.5 \mathrm{~mol} / \mathrm{lit}, \mathrm{C} 4=2 \mathrm{~mol} / \mathrm{lit}$ ). 


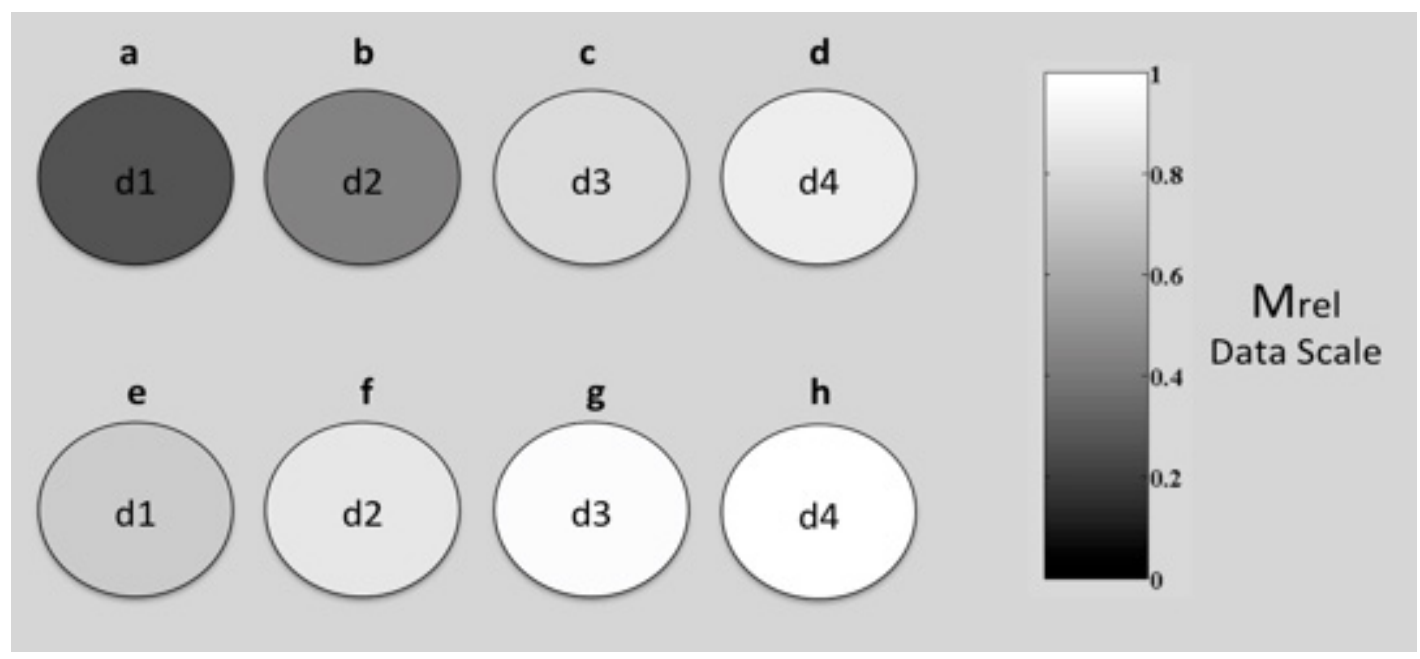

Figure 54: Showing the corresponding MRI/MPI signal for change in Relative magnetization (Mrel) at $\mathbf{H}=\mathbf{5 0}$ Oe for Concentrations. Mrel for magnetite (a-d). Mrel for MENs (e-f). (NOTE: (a,e at $=0.05 \mathrm{~mol} / \mathrm{lit}$ Concentration, b,f at $=0.1 \mathrm{~mol} / \mathrm{lit}$ Concentration, c,g at $=0.5 \mathrm{~mol} / \mathrm{lit}$ Concentration, $\mathrm{d}, \mathrm{h}$ at $=3 \mathrm{~mol} / \mathrm{lit}$ Concentration ).

\subsubsection{Brain segmentation}

Figure 55 shows a segmented MRI head data based on the conductive properties of the head.

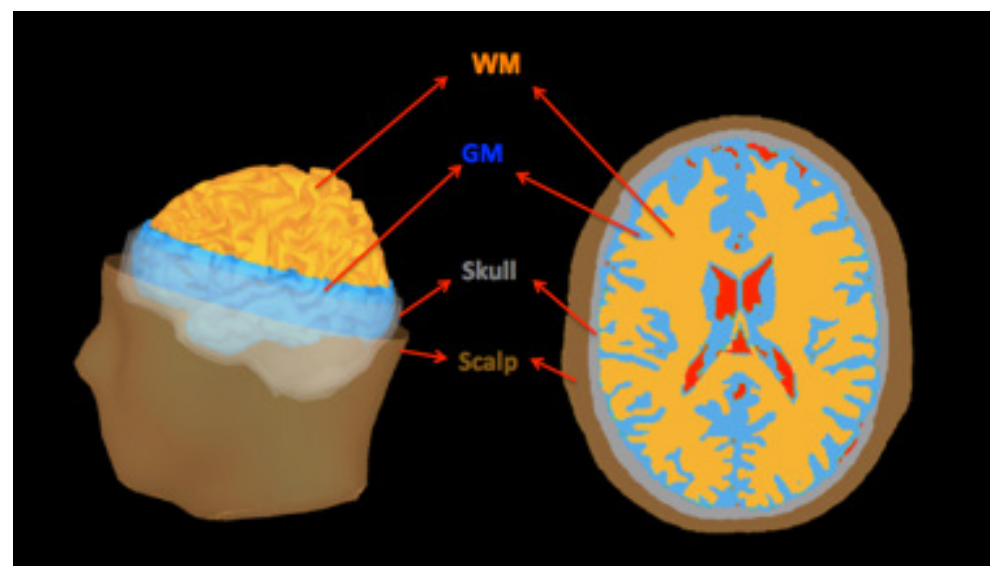

Figure 55: Segmented MRI data. White matter (orange), gray matter (blue), CSF (red), skull (gray), and scalp (brown). 


\subsubsection{Electrical activity mapping}

The electric field map was obtained for the neurons while firing with a current density of $\sim 70 \mathrm{~A} / \mathrm{F}$ (188) using the procedure described in the section 6.2.4. The results show the isotropic electric field for WM is $5 \mathrm{~V} / \mathrm{cm}^{-1}$, for GM is $2.1 \mathrm{~V} / \mathrm{cm}^{-1}$, and for CSF is $0 \mathrm{~V} / \mathrm{cm}^{-}$

${ }^{1}$. It should be noted that even though CSF exhibits higher conductivity $(1.79 \mathrm{~S} / \mathrm{m})$, the simulated electric field is $0 \mathrm{~V} / \mathrm{cm}^{-1}$, as neurons are absent in the CSF. Figure 56 shows the isotropic conductivity and electric field for different segments of the brain (assumption: all the neurons in the brain tissue are fired simultaneously).

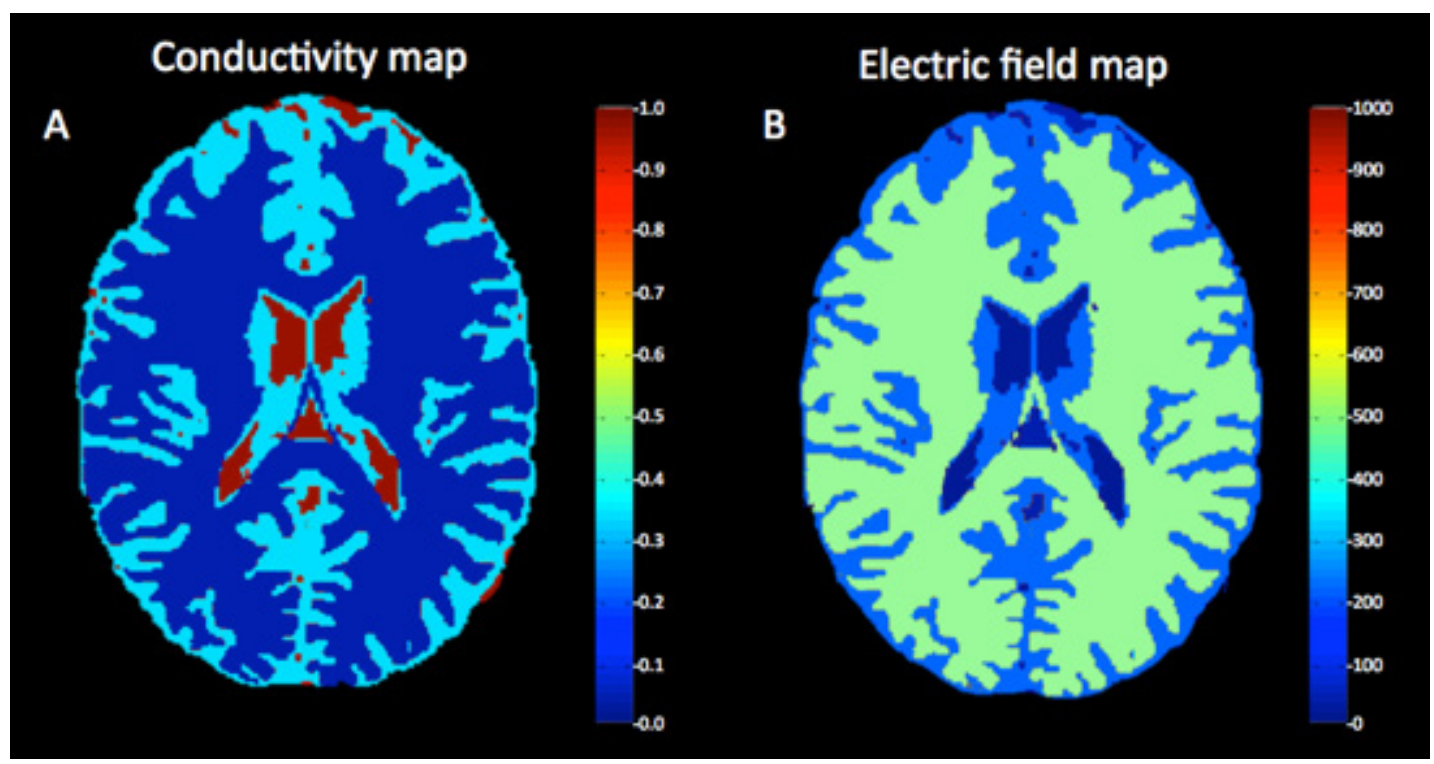

Figure 56: Isotropic electrical conductivity (A) and the electric field (B) map of the MRI segmented data.

\subsection{4. $\quad$ Tracking the neuronal activity}

Figure 57 show the neuronal activity of the brain in different regions based on the type of activity it performs. Figure 57A shows the regular MRI image based on the tissue type and the functions of the brain. Figure 57B through Figure 57E show the MRI image plus 
the electrical field image depending on the activities performed by the brain. It should be noted that by adding an additional signal generated solely by the change in the surface charge of the MENs gives additional information corresponding to the neuronal activities and further can be detected by the MRI/MNI imaging modality.

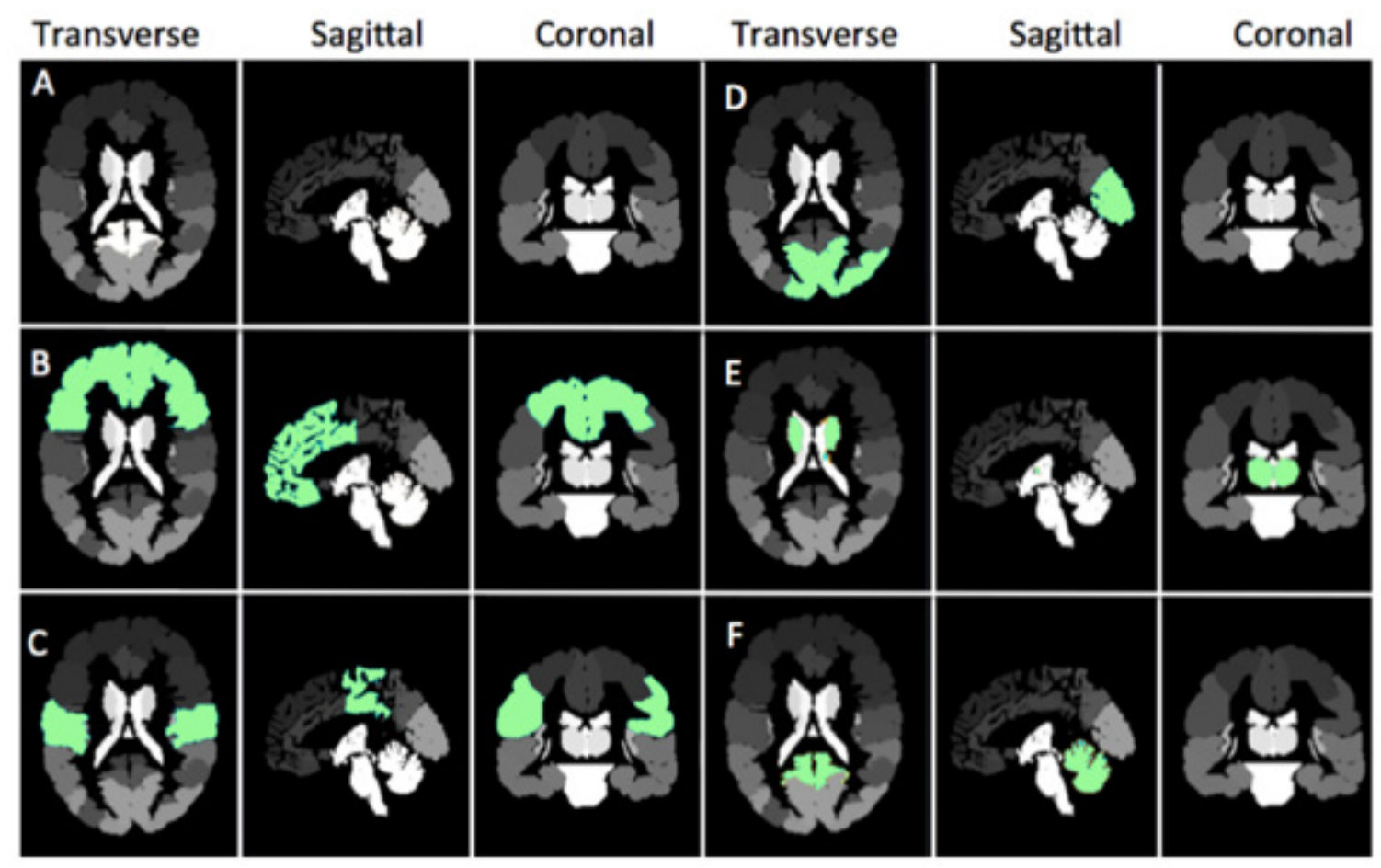

Figure 57: MRI image and electrical field image generated by using MENs as a contrasting agents. Images show a clear additional signal (green) solely generated by the change in surface charge of the MENs due to the change in membrane properties of the neurons during the propagation of the action potential.

\subsection{Discussion}

Figure 48 shows the MRI signal is strongly dependent on the surface charge of the MENs, as the relative magnetization of the MENs is significantly affected by the charge it carries (shown in Figure 46). Regular magnetic particle based contrasting agents do not show this unique phenomenon (Figure 47). We exploited this unique feature of the MENs to track the neuronal activity. The idea of using MENs for tracking neuronal activity is 
conceptualized in Figure 45. This figure clearly shows how MEN particle can change its relative magnetization in the vicinity of the action potential in the neurons. The relative magnetization is also affected by other properties, such as size and concentration of the contrasting agents. But such properties will not be influenced by the changes in the surrounding microenvironment and hence cannot be used, as contrasting agents for studying the molecular compositions of the surroundings. Figure 57 (B through E) shows the regular MRI signal (gray) image and an additional MRI signal (green) generated by the MENs contrasting agent. For example, when the neurons in the frontal lobes are fired (activities associated with the memory), the relative change in the magnetization of the MENs contrasting agents in the vicinity of the fired neurons provide additional signals shown in Figure 57B. Also, it should be noted that every activity (memory, vision, movement, and etc.) in the brain is performed by bunch of neurons (a minimum of $50,000)$ in a synchronous alpha rhythm rather than by a randomly fired single neuron (189). This property of the synchronous firing of neurons can further makes it possible to visualize neuronal activities using MRI/MNI imaging modalities. Moreover, most of the current MRI machines are capable of achieving close to microscale spatial resolutions $(190,191)$. Therefore, tracking these microenvironmental changes using MRI imaging modalities is fairly achievable. Using this approach one could track multiple activities related to brain. In this study, we showed that activities associated with frontal lobes like memory (Figure 57B), occipital lobes like vision (Figure 57D), parietal lobes like body movements (Figure 57C), cerebellum like co-ordination (Figure 57F), and thalamus like information process and relay (Figure 57E) can be tracked using MENs, as contrasting agents. 


\subsection{Conclusions}

In conclusion, we modeled the effect of surface charge of the MENs on the MRI/MNI signal intensities and compared it with the traditional contrasting agents. In this study we also presented a new concept of using these MENs, as contrasting agents to track the neuronal activity in the brain by MRI/MNI imaging modalities. The preliminary computational results of this study showed that using MENs, as contrasting agents, one could map neuronal activity independent of the origin (superficial or deep brain). This non-invasive brain mapping could benefit in understanding brain functions thoroughly in different mental and behavioral status, which is a key to treat several neurological diseases. 
Chapter 7: Conclusions and Future Work 


\subsection{Conclusions}

The ability to control the intrinsic electric properties of the magneto-electric nanoparticles using external magnetic field, provides several advantages to the fields of medicine (therapy and diagnosis). This dissertation aims to pave a way disease-specific medicine through a groundbreaking cross-disciplinary research in the fields of medicine, nano-engineering and physics to demonstrate the novel capabilities of magneto-electric nanoparticles (MENs) to enable unique mix of important functions, such as energyefficient and dissipation-free magnetic-field-controlled targeted drug delivery, ondemand release and high-specificity 3-D diagnostics. Specifically, MENs will be used for: (i) in-vitro delivery/release studies on anti-HIV drug AZTTP and anti-Cancer drug Paclitaxel, (ii) computational study on non-invasive targeted stimulation of CNS by lowenergy external magnetic field to treat Parkinson's Disease, Alzheimer's Disease, and other dementia, (iii) computation study to map the neuronal activity using MENs, as a highly sensitive MRI/MNI contrast agent.

Chapter 2 deals with the in vitro delivery/release study on anti-HIV drug AZTTP using magneto-electric nanoparticles (MENs), as carriers. Although highly active anti-retroviral therapy has resulted in remarkable decline in the morbidity and mortality in AIDS patients, inadequately low delivery of anti-retroviral drugs across the blood-brain barrier results in virus persistence. The capability of high-efficacy targeted drug delivery and ondemand release remains a formidable task. In this chapter we reported an in-vitro study to demonstrate the on-demand release of azidothymidine 5'-triphosphat, an anti-HIV drug, from 30-nm $\mathrm{CoFe}_{2} \mathrm{O}_{4} @ \mathrm{BaTiO}_{3}$ magneto-electric nanoparticles by applying a low a.c. magnetic field. Magneto-electric nanoparticles, as field-controlled drug carriers offer a 
unique capability of field-triggered release after crossing the blood-brain barrier. Due to the intrinsic magnetoelectricity, these nanoparticles can couple external magnetic fields with the electric forces in drug-carrier bonds to enable remotely controlled delivery without exploiting heat. Functional and structural integrity of the drug after the release was confirmed in in-vitro experiments with HIV-infected cells and through atomic force microscopy, spectrophotometry, FTIR and mass spectrometry studies.

Chapter 3 extends the application of MENs drug delivery technology to delivery a most widely used anti-cancer drug Paclitaxel (Taxol) for eradicating ovarian cancer cells. The development of a technology capable of high-specificity targeted delivery of antineoplastic drugs would be a significant breakthrough in Cancer in general and Ovarian Cancer in particular. We addressed this challenge through a nanotechnology that exploited (i) the difference in the membrane electric properties between the tumor and healthy cells and (ii) the capability of magneto-electric nanoparticles (MENs) to serve, as nanosized converters of remote magnetic field energy into the MENs' intrinsic electric field energy. This capability allows to remotely control the electric fields within the cell membranes and thus trigger high-specificity uptake of the drug through generation of localized nano-electroporation sites in the tumor cells only. In in-vitro studies on human ovarian carcinoma cell (SKOV-3) and healthy ovarian cell (HOMEC) lines, we triggered high-specificity uptake of paclitaxel that was loaded on 30-nm $\mathrm{CoFe}_{2} \mathrm{O}_{4} @ \mathrm{BaTiO}_{3} \mathrm{MENs}$ by a 30-Oe d.c. magnetic field. The drug penetrated through the membrane and completely eradicated the tumor cells within 24 hours without affecting the healthy cells. The conclusions were supported by atomic force microscopy, Fourier-transform infra-red 
and X-ray diffraction analyses, mass spectrometry, surface temperature measurements, and fluorescence imaging.

Chapter 4 involves in developing MENs technology for targeted drug delivery and ondemand field-controlled release can overcome the control challenges of the conventional delivery approaches. The magneto-electric effect allows to use an external magnetic field to remotely connect to the intrinsic electric fields that govern the binding forces between the functionalized MENs' surface and the drug load. Herein we report how tailoring the composition of the intermediate functionalized layer can be used to control the threshold magnetic field for the dissociation of the drug from 30-nm $\mathrm{CoFe}_{2} \mathrm{O}_{4}-\mathrm{BaTiO}_{3}$ coreshell MENs in a controllably wide field range, from below 10 to over 200 Oe, as required for enabling superficial, intermediate, and deep-tissue drug delivery. Paclitaxel is used, as the model drug. Specific experiments are described to achieve controllable dissociation of the drug molecules from the MENs' surface at the following three different sub-ranges, (i) low (<10 Oe), (ii) moderate (100 Oe), and (iii) high ( $>200$ Oe), respectively, by selecting the following 2-nm intermediate layers, (i) Glycerol monooleate (GMO), (ii) Tween-20, and (iii) Ethyl-3-(3-dimethylaminopropyl)carbodiimide (EDC), respectively. Toxicity study on SKOV-3 cell lines using XTT assay at the particle concentration of 50 $\mu \mathrm{g} / \mathrm{ml}$ resulted in the following percentages of viable cells: (i) non-functionalized MENs: 96\%, (ii) GMO-MENs: $100 \%$, (iii) Tween-20-MENs: 89\%, (iii) EDC-MENs: $77 \%$. Fielddependent FTIR, fluorescence imaging, absorption spectra, atomic force microscopy, magnetometry analysis, zeta-potential measurements, and blood circulation experiments are used to study the described functionalization effects. 
Chapter 5 describes a computational study of using MENs to artificially stimulate the neural activity deep in the brain. The new technology provides a unique way to couple electric signals in the neural network to the magnetic dipoles in the nanoparticles with the purpose to enable a non-invasive approach. Simulations of the effect of MENs for noninvasively stimulating the brain of a patient with Parkinson's Disease to bring the pulsed sequences of the electric field to the levels comparable to those of healthy people show that the optimized values for the concentration of the 20-nm nanoparticles (with the magneto-electric (ME) coefficient of $100 \mathrm{~V} \mathrm{~cm}^{-1} \mathrm{Oe}^{-1}$ in the aqueous solution) is $3 \times 10^{6}$ particles/cc, and the frequency of the externally applied 300-Oe magnetic field is $80 \mathrm{~Hz}$.

Chapter 6 presents a computational study of MENs for tracking the neuronal activities in the brain using imaging modalities, such as MRI and MNI. In this chapter a unique property of the MENs, i.e. magnetization dependence on its surface charge is computed to account for the change in the MR/MN signal intensity. Based on this property we computed the electric fields in different regions of the brain for the neuronal current density of $70 \mathrm{pA} / \mathrm{pF}$ and found that the active regions of brain increases MRI signal by $30 \%$ for $50 \mathrm{~nm}$ sized MENs with a ME coefficient $100 \mathrm{~V} \mathrm{~cm}^{-1} \mathrm{Oe}^{-1}$ at the concentration of $0.5 \mathrm{~mol} / \mathrm{L}$.

\subsection{Future Work}

The MENs based drug delivery/release of anti-HIV drug (AZTTP) will be further evaluated in the in vivo HIV-infected monkey models (Rhesus macaque) at Yerkes 
National Primate Research Center of Emory University, Georgia. The MENs based drug delivery/release of anti-cancer drug (Taxol) will be further evaluated in the in vivo ovarian cancer mice model at animal care facility of Florida International University, Florida. MENs technology for stimulating the neuronal activity will be further evaluated in the in vivo Parkinson's mice model at animal care facility of Florida International University, Florida. 


\section{REFERENCES}

1. M. Nair et al., Externally controlled on-demand release of anti-HIV drug using magneto-electric nanoparticles as carriers. Nature communications 4, 1707 (2013).

2. U. Pison, T. Welte, M. Giersig, D. A. Groneberg, Nanomedicine for respiratory diseases. European Journal of Pharmacology 533, 341 (2006).

3. L. Brannon-Peppas, J. O. Blanchette, Nanoparticle and targeted systems for cancer therapy. Advanced drug delivery reviews, (2012).

4. G. K. Stylios, P. V. Giannoudis, T. Wan, Applications of nanotechnologies in medical practice. Injury 36, S6 (2005).

5. M. Yokoyama, Drug targeting with nano-sized carrier systems. Journal of Artificial Organs 8, 77 (2005).

6. A. G. Schätzlein, Delivering cancer stem cell therapies-A role for nanomedicines? European Journal of Cancer 42, 1309 (2006).

7. N. T. K. Thanh, Z. Rosenzweig, Development of an aggregation-based immunoassay for anti-protein A using gold nanoparticles. Analytical chemistry 74, 1624 (2002).

8. C. A. Mirkin, R. L. Letsinger, R. C. Mucic, J. J. Storhoff, A DNA-based method for rationally assembling nanoparticles into macroscopic materials. (1996).

9. H. Li, L. Rothberg, Colorimetric detection of DNA sequences based on electrostatic interactions with unmodified gold nanoparticles. Proceedings of the National Academy of Sciences of the United States of America 101, 14036 (2004).

10. K. Sato, K. Hosokawa, M. Maeda, Rapid aggregation of gold nanoparticles induced by non-cross-linking DNA hybridization. Journal of the American Chemical Society 125, 8102 (2003).

11. Y. C. Cao, R. Jin, C. A. Mirkin, Nanoparticles with Raman spectroscopic fingerprints for DNA and RNA detection. Science 297, 1536 (2002).

12. G. Han, P. Ghosh, V. M. Rotello, Functionalized gold nanoparticles for drug delivery. Nanomedicine 2, 113 (2007).

13. S. Dhar, E. M. Reddy, A. Shiras, V. Pokharkar, B. e. L. e. V. Prasad, Natural gum reduced/stabilized gold nanoparticles for drug delivery formulations. Chemistry-A European Journal 14, 10244 (2008). 
14. L. Song et al., Efficient, $\mathrm{pH}$ - Triggered Drug Delivery Using a $\mathrm{pH}$ - Responsive DNA - Conjugated Gold Nanoparticle. Advanced healthcare materials, (2012).

15. A. S. Angelatos, B. Radt, F. Caruso, Light-responsive polyelectrolyte/gold nanoparticle microcapsules. The Journal of Physical Chemistry B 109, 3071 (2005).

16. G. Han et al., Light - Regulated Release of DNA and Its Delivery to Nuclei by Means of Photolabile Gold Nanoparticles. Angewandte Chemie 118, 3237 (2006).

17. L. Poon et al., Photothermal Release of Single-Stranded DNA from the Surface of Gold Nanoparticles Through Controlled Denaturating and $\mathrm{Au}-\mathrm{S}$ Bond Breaking. ACS nano 4, 6395 (2010).

18. S. H. Cho, B. L. Jones, S. Krishnan, The dosimetric feasibility of gold nanoparticle-aided radiation therapy (GNRT) via brachytherapy using low-energy gamma-/x-ray sources. Physics in medicine and biology 54, 4889 (2009).

19. I. H. El-Sayed, X. Huang, M. A. El-Sayed, Selective laser photo-thermal therapy of epithelial carcinoma using anti-EGFR antibody conjugated gold nanoparticles. Cancer letters 239, 129 (2006).

20. X. Huang, P. K. Jain, I. H. El-Sayed, M. A. El-Sayed, Plasmonic photothermal therapy (PPTT) using gold nanoparticles. Lasers in medical science 23, 217 (2008).

21. C. Loo, A. Lowery, N. Halas, J. West, R. Drezek, Immunotargeted nanoshells for integrated cancer imaging and therapy. Nano letters 5, 709 (2005).

22. J. Hainfeld et al., Micro-CT enables microlocalisation and quantification of Her2targeted gold nanoparticles within tumour regions. British Journal of Radiology 84, 526 (2011).

23. X. Huang, I. H. El-Sayed, W. Qian, M. A. El-Sayed, Cancer cell imaging and photothermal therapy in the near-infrared region by using gold nanorods. Journal of the American Chemical Society 128, 2115 (2006).

24. P. K. Jain, K. S. Lee, I. H. El-Sayed, M. A. El-Sayed, Calculated absorption and scattering properties of gold nanoparticles of different size, shape, and composition: applications in biological imaging and biomedicine. The Journal of Physical Chemistry B 110, 7238 (2006).

25. X. Liu, M. Atwater, J. Wang, Q. Huo, Extinction coefficient of gold nanoparticles with different sizes and different capping ligands. Colloids and Surfaces B: Biointerfaces 58, 3 (2007). 
26. Y. Sun, Y. Xia, Shape-controlled synthesis of gold and silver nanoparticles. Science 298, 2176 (2002).

27. N. R. Jana, L. Gearheart, C. J. Murphy, Wet chemical synthesis of high aspect ratio cylindrical gold nanorods. The Journal of Physical Chemistry B 105, 4065 (2001).

28. R. Goel, N. Shah, R. Visaria, G. F. Paciotti, J. C. Bischof, Biodistribution of TNF- $\alpha$-coated gold nanoparticles in an in vivo model system. Nanomedicine $\mathbf{4}$, 401 (2009).

29. E. I. Altınog $` u$ et al., Near-infrared emitting fluorophore-doped calcium phosphate nanoparticles for in vivo imaging of human breast cancer. ACS nano $\mathbf{2}$, 2075 (2008).

30. G. Zhang et al., Influence of anchoring ligands and particle size on the colloidal stability and $<\mathrm{i}>$ in vivo $<\mathrm{i}>$ biodistribution of polyethylene glycol-coated gold nanoparticles in tumor-xenografted mice. Biomaterials 30, 1928 (2009).

31. J. Hainfeld, D. Slatkin, T. Focella, H. Smilowitz, Gold nanoparticles: a new X-ray contrast agent. British Journal of Radiology 79, 248 (2006).

32. Y. Cheng et al., Highly efficient drug delivery with gold nanoparticle vectors for in vivo photodynamic therapy of cancer. Journal of the American Chemical Society 130, 10643 (2008).

33. R. Gilchrist et al., Selective inductive heating of lymph nodes. Annals of Surgery 146, 596 (1957).

34. J. L. Kirschvink, M. M. Walker, C. E. Diebel, Magnetite-based magnetoreception. Current opinion in neurobiology 11, 462 (2001).

35. D. Dunlop, Superparamagnetic and single-domain threshold sizes in magnetite. Journal of Geophysical Research 78, 1780 (1973).

36. Z. Li, L. Wei, M. Gao, H. Lei, One - Pot Reaction to Synthesize Biocompatible Magnetite Nanoparticles. Advanced Materials 17, 1001 (2005).

37. M. Strömberg et al., Interbead interactions within oligonucleotide functionalized ferrofluids suitable for magnetic biosensor applications. Journal of Physics D: Applied Physics 40, 1320 (2007).

38. Y. Sahoo et al., Aqueous ferrofluid of magnetite nanoparticles: fluorescence labeling and magnetophoretic control. The Journal of Physical Chemistry B 109, 3879 (2005). 
39. A. Jordan et al., Presentation of a new magnetic field therapy system for the treatment of human solid tumors with magnetic fluid hyperthermia. Journal of Magnetism and Magnetic Materials 225, 118 (2001).

40. S. Hosseini, M. Mehrtash, M. B. Khamesee, Design, fabrication and control of a magnetic capsule-robot for the human esophagus. Microsystem technologies 17, 1145 (2011).

41. R. Probst et al., Planar steering of a single ferrofluid drop by optimal minimum power dynamic feedback control of four electromagnets at a distance. Journal of magnetism and magnetic materials 323, 885 (2011).

42. W. S. Enochs, G. Harsh, F. Hochberg, R. Weissleder, Improved delineation of human brain tumors on MR images using a long - circulating, superparamagnetic iron oxide agent. Journal of Magnetic Resonance Imaging 9, 228 (1999).

43. U. Schoepf, E. Marecos, R. Melder, R. Jain, R. Weissleder, Intracellular magnetic labeling of lymphocytes for in vivo trafficking studies. Biotechniques 24, 642 (1998).

44. P. Cuatrecasas, T. Roth, Receptor-mediated endocytosis. (Chapman and Hall, 1983), vol. 15.

45. R. Weissleder, H. C. Cheng, A. Bogdanova, A. Bogdanov, Magnetically labeled cells can be detected by MR imaging. Journal of Magnetic Resonance Imaging 7, 258 (1997).

46. A. K. Gupta, M. Gupta, Synthesis and surface engineering of iron oxide nanoparticles for biomedical applications. Biomaterials 26, 3995 (2005).

47. B. Lobel, O. Eyal, N. Kariv, A. Katzir, Temperature controlled CO2 laser welding of soft tissues: Urinary bladder welding in different animal models (rats, rabbits, and cats). Lasers in surgery and medicine 26, 4 (2000).

48. P. Jendelová et al., Magnetic resonance tracking of transplanted bone marrow and embryonic stem cells labeled by iron oxide nanoparticles in rat brain and spinal cord. Journal of neuroscience research 76, 232 (2004).

49. J. Dobson, Magnetic nanoparticles for drug delivery. Drug Development Research 67, 55 (2006).

50. B. Pan et al., Dendrimer-modified magnetic nanoparticles enhance efficiency of gene delivery system. Cancer research 67, 8156 (2007).

51. X. Luo, S. Liu, J. Zhou, L. Zhang, In situ synthesis of Fe3O4/cellulose microspheres with magnetic-induced protein delivery. Journal of Materials Chemistry 19, 3538 (2009). 
52. G. Storm, S. O. Belliot, T. Daemen, D. D. Lasic, Surface modification of nanoparticles to oppose uptake by the mononuclear phagocyte system. Advanced Drug Delivery Reviews 17, 31 (1995).

53. S.-J. Lee et al., Nanoparticles of magnetic ferric oxides encapsulated with poly (D, L latide-co-glycolide) and their applications to magnetic resonance imaging contrast agent. Journal of magnetism and magnetic materials 272, 2432 (2004).

54. L. Wang, K.-G. Neoh, E.-T. Kang, B. Shuter, S.-C. Wang, Biodegradable magnetic-fluorescent magnetite/poly (dl-lactic acid-co- $<\mathrm{i}>\alpha</ \mathrm{i}>,<\mathrm{i}>\beta</ \mathrm{i}>$ malic acid) composite nanoparticles for stem cell labeling. Biomaterials 31, 3502 (2010).

55. C. Corot, P. Robert, J.-M. Idée, M. Port, Recent advances in iron oxide nanocrystal technology for medical imaging. Advanced drug delivery reviews $\mathbf{5 8}$, 1471 (2006).

56. M. Lewin et al., Tat peptide-derivatized magnetic nanoparticles allow in vivo tracking and recovery of progenitor cells. Nature biotechnology 18, 410 (2000).

57. R. Weissleder et al., In vivo magnetic resonance imaging of transgene expression. Nature medicine 6, 351 (2000).

58. M. Zhao, D. A. Beauregard, L. Loizou, B. Davletov, K. M. Brindle, Non-invasive detection of apoptosis using magnetic resonance imaging and a targeted contrast agent. Nature medicine 7, 1241 (2001).

59. O. S. Nielsen, M. Horsman, J. Overgaard, A future for hyperthermia in cancer treatment? European Journal of Cancer 37, 1587 (2001).

60. L. Miller, J. Leor, B. Rubinsky, Cancer cells ablation with irreversible electroporation. Technology in cancer research \& treatment 4, 699 (2005).

61. M. Abe et al., Multi - institutional studies on hyperthermia using an $8-\mathrm{MHz}$ radiofrequency capacitive heating device (thermotron RF - 8) in combination with radiation for cancer therapy. Cancer 58, 1589 (1986).

62. A. Jordan, R. Scholz, P. Wust, H. Fähling, R. Felix, Magnetic fluid hyperthermia (MFH): Cancer treatment with $\mathrm{AC}$ magnetic field induced excitation of biocompatible superparamagnetic nanoparticles. Journal of Magnetism and Magnetic Materials 201, 413 (1999).

63. M. Johannsen et al., Clinical hyperthermia of prostate cancer using magnetic nanoparticles: presentation of a new interstitial technique. International Journal of Hyperthermia 21, 637 (2005). 
64. W. Eerenstein, N. Mathur, J. F. Scott, Multiferroic and magnetoelectric materials. Nature 442, 759 (2006).

65. E. Ascher, H. Rieder, H. Schmid, H. Stössel, Some Properties of Ferromagnetoelectric Nickel - Iodine Boracite, NiBOI. Journal of Applied Physics 37, 1404 (1966).

66. J. Van den Boomgaard, D. Terrell, R. Born, H. Giller, An in situ grown eutectic magnetoelectric composite material. Journal of Materials Science 9, 1705 (1974).

67. C.-W. Nan et al., A three-phase magnetoelectric composite of piezoelectric ceramics, rare-earth iron alloys, and polymer. Applied Physics Letters 81, 3831 (2002).

68. N. Cai, C.-W. Nan, J. Zhai, Y. Lin, Large high-frequency magnetoelectric response in laminated composites of piezoelectric ceramics, rare-earth iron alloys and polymer. Applied physics letters 84, 3516 (2004).

69. J. Ryu, A. V. Carazo, K. Uchino, H.-E. Kim, Magnetoelectric properties in piezoelectric and magnetostrictive laminate composites. Japanese Journal of Applied Physics 40, 4948 (2001).

70. G. Srinivasan et al., Magnetoelectric bilayer and multilayer structures of magnetostrictive and piezoelectric oxides. Physical Review B 64, 214408 (2001).

71. L. Bracke, R. Van Vliet, A broadband magneto-electric transducer using a composite material. International Journal of Electronics Theoretical and Experimental 51, 255 (1981).

72. R. Petrov et al., Miniature antenna based on magnetoelectric composites. Electronics Letters 44, 506 (2008).

73. J. Scott, Data storage: Multiferroic memories. Nature materials 6, 256 (2007).

74. A. Khitun, D. E. Nikonov, K. L. Wang, Magnetoelectric spin wave amplifier for spin wave logic circuits. Journal of Applied Physics 106, 123909 (2009).

75. P. Li, Y. Wen, P. Liu, X. Li, C. Jia, A magnetoelectric energy harvester and management circuit for wireless sensor network. Sensors and actuators A: Physical 157, 100 (2010).

76. X. Chen, A. Hochstrat, P. Borisov, W. Kleemann, Magnetoelectric exchange bias systems in spintronics. Applied physics letters 89, 202508 (2006).

77. N. Kida et al., Optical magnetoelectric effect of patterned oxide superlattices with ferromagnetic interfaces. Physical review letters 99, 197404 (2007). 
78. P. Blomstedt, M. I. Hariz, Are complications less common in deep brain stimulation than in ablative procedures for movement disorders? Stereotactic and functional neurosurgery 84, 72 (2006).

79. A. Alkhani, A. M. Lozano, Pallidotomy for Parkinson disease: a review of contemporary literature. Journal of neurosurgery 94, 43 (2001).

80. D. Fontaine et al., Safety and efficacy of deep brain stimulation in refractory cluster headache: a randomized placebo-controlled double-blind trial followed by a 1-year open extension. The journal of headache and pain 11, 23 (2010).

81. H. S. Mayberg et al., Deep brain stimulation for treatment-resistant depression. Neuron 45, 651 (2005).

82. K. Kumar, C. Toth, R. K. Nath, Deep brain stimulation for intractable pain: a 15year experience. Neurosurgery 40, 736 (1997).

83. A. M. Lozano et al., Subcallosal cingulate gyrus deep brain stimulation for treatment-resistant depression. Biological psychiatry 64, 461 (2008).

84. J. S. Perlmutter, J. W. Mink, Deep brain stimulation. Annu. Rev. Neurosci. 29, 229 (2006).

85. F. C. Hummel, L. G. Cohen, Non-invasive brain stimulation: a new strategy to improve neurorehabilitation after stroke? The Lancet Neurology 5, 708 (2006).

86. P. C. Gandiga, F. C. Hummel, L. G. Cohen, Transcranial DC stimulation (tDCS): a tool for double-blind sham-controlled clinical studies in brain stimulation. Clinical Neurophysiology 117, 845 (2006).

87. T. Wagner, A. Valero-Cabre, A. Pascual-Leone, Noninvasive human brain stimulation. Annu. Rev. Biomed. Eng. 9, 527 (2007).

88. S. Sanei, J. A. Chambers, EEG signal processing. (Wiley. com, 2008).

89. G. Aston-Jones, F. Bloom, Activity of norepinephrine-containing locus coeruleus neurons in behaving rats anticipates fluctuations in the sleep-waking cycle. The Journal of Neuroscience 1, 876 (1981).

90. S. J. Lewis, A. Dove, T. W. Robbins, R. A. Barker, A. M. Owen, Cognitive impairments in early Parkinson's disease are accompanied by reductions in activity in frontostriatal neural circuitry. The Journal of Neuroscience 23, 6351 (2003).

91. J. W. Phillis, L. A. Horrocks, A. A. Farooqui, Cyclooxygenases, lipoxygenases, and epoxygenases in CNS: their role and involvement in neurological disorders. Brain research reviews 52, 201 (2006). 
92. D. Paré, E. Shink, H. Gaudreau, A. Destexhe, E. J. Lang, Impact of spontaneous synaptic activity on the resting properties of cat neocortical pyramidal neurons in vivo. Journal of neurophysiology 79, 1450 (1998).

93. D. B. Lindsley, J. Bowden, H. Magoun, Effect upon the EEG of acute injury to the brain stem activating system. Electroencephalography and clinical neurophysiology 1, 475 (1949).

94. B. P. Timko, T. Dvir, D. S. Kohane, Remotely triggerable drug delivery systems. Advanced Materials 22, 4925 (2010).

95. Z. M. Saiyed, N. H. Gandhi, M. P. Nair, Magnetic nanoformulation of azidothymidine 5'-triphosphate for targeted delivery across the blood-brain barrier. International journal of nanomedicine 5, 157 (2010).

96. E. V. Batrakova, H. E. Gendelman, A. V. Kabanov, Cell-mediated drug delivery. Expert opinion on drug delivery 8, 415 (2011).

97. K. Yue et al., Magneto-electric nano-particles for non-invasive brain stimulation. PloS one 7, e44040 (2012).

98. A. Mocroft, J. D. Lundgren, Starting highly active antiretroviral therapy: why, when and response to HAART. Journal of Antimicrobial Chemotherapy 54, 10 (2004).

99. X. Wang, H. Chai, P. H. Lin, Q. Yao, C. Chen, Roles and mechanisms of human immunodeficiency virus protease inhibitor ritonavir and other anti-human immunodeficiency virus drugs in endothelial dysfunction of porcine pulmonary arteries and human pulmonary artery endothelial cells. The American journal of pathology 174, 771 (2009).

100. S. K. Saxena, S. Tiwari, M. P. Nair, A global perspective on HIV/AIDS. Science 337, 798 (2012).

101. S. Ayre, New approaches to the delivery of drugs to the brain. Medical Hypotheses 29, 283 (1989).

102. S. Thomas, Anti-HIV drug distribution to the central nervous system. Current pharmaceutical design 10, 1313 (2004).

103. R. Tong, H. D. Hemmati, R. Langer, D. S. Kohane, Photoswitchable nanoparticles for triggered tissue penetration and drug delivery. Journal of the American Chemical Society 134, 8848 (2012).

104. I. Cheong et al., A bacterial protein enhances the release and efficacy of liposomal cancer drugs. Science 314, 1308 (2006). 
105. A. Senyei, K. Widder, G. Czerlinski, Magnetic guidance of drug - carrying microspheres. Journal of Applied Physics 49, 3578 (1978).

106. A. M. Derfus et al., Remotely triggered release from magnetic nanoparticles. Advanced Materials 19, 3932 (2007).

107. S. C. McBain, H. H. Yiu, J. Dobson, Magnetic nanoparticles for gene and drug delivery. International Journal of Nanomedicine 3, 169 (2008).

108. J. SZEBENI et al., Inhibition of HIV-1 in Monocyte/Macrophage Cultures by $2^{\prime}$ , 3' -Dideoxycytidine-5' -Triphosphate, Free and in Liposomes*. AIDS research and human retroviruses 6, 691 (1990).

109. L. Varatharajan, S. A. Thomas, The transport of anti-HIV drugs across bloodCNS interfaces: summary of current knowledge and recommendations for further research. Antiviral research 82, A99 (2009).

110. S. Xie, F. Ma, Y. Liu, J. Li, Multiferroic CoFe2O4-Pb (Zr0. 52Ti0. 48) O3 coreshell nanofibers and their magnetoelectric coupling. Nanoscale 3, 3152 (2011).

111. Y. Persidsky et al., A model for monocyte migration through the blood-brain barrier during HIV-1 encephalitis. The Journal of Immunology 158, 3499 (1997).

112. V. Corral-Flores, D. Bueno-Baques, R. Ziolo, Synthesis and characterization of novel $\mathrm{CoFe}<\mathrm{sub}>2</$ sub $>\mathrm{O}<\mathrm{sub}>4</$ sub $>-\mathrm{BaTiO}<\mathrm{sub}>3</$ sub $>$ multiferroic core-shell-type nanostructures. Acta Materialia 58, 764 (2010).

113. J. Litvinov et al., High-Throughput Top-Down Fabrication of Uniform Magnetic Particles. PloS one 7, e37440 (2012).

114. J. Hong et al., Room-temperature Magnetic Ordering in Functionalized Graphene. Scientific reports 2, (2012).

115. S. A. Rovers, R. Hoogenboom, M. F. Kemmere, J. T. Keurentjes, Repetitive ondemand drug release by magnetic heating of iron oxide containing polymeric implants. Soft Matter 8, 1623 (2012).

116. T. Hoare et al., A magnetically triggered composite membrane for on-demand drug delivery. Nano letters 9, 3651 (2009).

117. K. Chan, C. Koh, H. Li, Mitosis-targeted anti-cancer therapies: where they stand. Cell death \& disease 3, e411 (2012).

118. J. K. Vasir, V. Labhasetwar, Targeted drug delivery in cancer therapy. Technology in cancer research \& treatment 4, 363 (2005). 
119. M. Yoshida et al., Targeting Anticancer Drug Delivery to Pancreatic Cancer Cells Using a Fucose-Bound Nanoparticle Approach. PloS one 7, e39545 (2012).

120. D. K. Armstrong et al., Intraperitoneal cisplatin and paclitaxel in ovarian cancer. New England Journal of Medicine 354, 34 (2006).

121. R. K. Oldham, Monoclonal antibodies in cancer therapy. Journal of Clinical Oncology 1, 582 (1983).

122. M. Bichurin, V. Petrov, G. Srinivasan, Theory of low-frequency magnetoelectric coupling in magnetostrictive-piezoelectric bilayers. Physical Review B 68, 054402 (2003).

123. T. Kimura et al., Magnetic control of ferroelectric polarization. Nature 426, 55 (2003).

124. D. Wang, W. Goh, M. Ning, C. Ong, Effect of Ba doping on magnetic, ferroelectric, and magnetoelectric properties in mutiferroic $\mathrm{BiFeO}$ at room temperature. Applied physics letters 88, 212907 (2006).

125. S. Valencia et al., Interface-induced room-temperature multiferroicity in BaTiO3. Nature materials 10, 753 (2011).

126. T. Zhao et al., Electrical control of antiferromagnetic domains in multiferroic $\mathrm{BiFeO} 3$ films at room temperature. Nature materials 5, 823 (2006).

127. S. Ho, G. Mittal, Electroporation of cell membranes: a review. Critical reviews in biotechnology 16, 349 (1996).

128. R. Binggeli, I. L. Cameron, Cellular potentials of normal and cancerous fibroblasts and hepatocytes. Cancer research 40, 1830 (1980).

129. M. A. Bookman, Update of randomized trials in first-line treatment. Annals of Oncology 22, Viii52 (2011).

130. P. Kocbek, N. Obermajer, M. Cegnar, J. Kos, J. Kristl, Targeting cancer cells using PLGA nanoparticles surface modified with monoclonal antibody. Journal of Controlled Release 120, 18 (2007).

131. P. Kesarwani, R. K. Tekade, N. Jain, Spectrophotometric estimation of paclitaxel. International Journal of Advances in Pharmaceutical Sciences 2, (2011).

132. S. Douglas, S. Davis, L. Illum, Nanoparticles in drug delivery. Critical reviews in therapeutic drug carrier systems 3, 233 (1987). 
133. K. S. Soppimath, T. M. Aminabhavi, A. R. Kulkarni, W. E. Rudzinski, Biodegradable polymeric nanoparticles as drug delivery devices. Journal of controlled release 70, 1 (2001).

134. S. A. Agnihotri, N. N. Mallikarjuna, T. M. Aminabhavi, Recent advances on chitosan-based micro-and nanoparticles in drug delivery. Journal of Controlled Release 100, 5 (2004).

135. I. Slowing, B. G. Trewyn, V. S.-Y. Lin, Effect of surface functionalization of MCM-41-type mesoporous silica nanoparticles on the endocytosis by human cancer cells. Journal of the American Chemical Society 128, 14792 (2006).

136. Y. Zhang, N. Kohler, M. Zhang, Surface modification of superparamagnetic magnetite nanoparticles and their intracellular uptake. Biomaterials 23, 1553 (2002).

137. H. Gu, Z. Yang, J. Gao, C. Chang, B. Xu, Heterodimers of nanoparticles: Formation at a liquid-liquid interface and particle-specific surface modification by functional molecules. Journal of the American Chemical Society 127, 34 (2005).

138. C. Coester, K. Langer, H. Von Briesen, J. Kreuter, Gelatin nanoparticles by two step desolvation a new preparation method, surface modifications and cell uptake. Journal of microencapsulation 17, 187 (2000).

139. T. Neuberger, B. Schöpf, H. Hofmann, M. Hofmann, B. von Rechenberg, Superparamagnetic nanoparticles for biomedical applications: possibilities and limitations of a new drug delivery system. Journal of Magnetism and Magnetic Materials 293, 483 (2005).

140. A. zur Mühlen, C. Schwarz, W. Mehnert, Solid lipid nanoparticles (SLN) for controlled drug delivery-drug release and release mechanism. European journal of pharmaceutics and biopharmaceutics 45, 149 (1998).

141. T. K. Jain, M. A. Morales, S. K. Sahoo, D. L. Leslie-Pelecky, V. Labhasetwar, Iron oxide nanoparticles for sustained delivery of anticancer agents. Molecular pharmaceutics 2, 194 (2005).

142. C.-Y. Lin, C.-J. Yu, Y.-H. Lin, W.-L. Tseng, Colorimetric sensing of silver (I) and mercury (II) ions based on an assembly of Tween 20-stabilized gold nanoparticles. Analytical chemistry 82, 6830 (2010).

143. F. Dilnawaz, A. Singh, C. Mohanty, S. K. Sahoo, Dual drug loaded superparamagnetic iron oxide nanoparticles for targeted cancer therapy. Biomaterials 31, 3694 (2010).

144. M. Babic et al., Poly (L-lysine)-modified iron oxide nanoparticles for stem cell labeling. Bioconjugate chemistry 19, 740 (2008). 
145. J. R. McCarthy, R. Weissleder, Multifunctional magnetic nanoparticles for targeted imaging and therapy. Advanced drug delivery reviews 60, 1241 (2008).

146. M. J. Heffernan, N. Murthy, Polyketal nanoparticles: a new pH-sensitive biodegradable drug delivery vehicle. Bioconjugate chemistry 16, 1340 (2005).

147. I. I. Slowing, B. G. Trewyn, S. Giri, V. Y. Lin, Mesoporous silica nanoparticles for drug delivery and biosensing applications. Advanced Functional Materials 17, 1225 (2007).

148. S. Sershen, S. Westcott, N. Halas, J. West, Temperature - sensitive polymernanoshell composites for photothermally modulated drug delivery. Journal of biomedical materials research 51, 293 (2000).

149. Q. A. Pankhurst, J. Connolly, S. Jones, J. Dobson, Applications of magnetic nanoparticles in biomedicine. Journal of physics D: Applied physics 36, R167 (2003).

150. A. Sunters et al., FoxO3a transcriptional regulation of Bim controls apoptosis in paclitaxel-treated breast cancer cell lines. Journal of Biological Chemistry 278, 49795 (2003).

151. E. E. Vokes et al., Weekly carboplatin and paclitaxel followed by concomitant paclitaxel, fluorouracil, and hydroxyurea chemoradiotherapy: curative and organpreserving therapy for advanced head and neck cancer. Journal of Clinical Oncology 21, 320 (2003).

152. R. Mahajan et al., Magnetoelectric effect in cobalt ferrite-barium titanate composites and their electrical properties. Pramana 58, 1115 (2002).

153. Y. Zhang, C. Deng, J. Ma, Y. Lin, C.-W. Nan, Enhancement in magnetoelectric response in CoFe $2 \mathrm{O} 4-\mathrm{BaTiO} 3$ heterostructure. Applied Physics Letters 92, 062911 (2008).

154. W. Geldenhuys, T. Mbimba, T. Bui, K. Harrison, V. Sutariya, Brain-targeted delivery of paclitaxel using glutathione-coated nanoparticles for brain cancers. Journal of Drug Targeting 19, 837 (2011).

155. N. W. Roehm, G. H. Rodgers, S. M. Hatfield, A. L. Glasebrook, An improved colorimetric assay for cell proliferation and viability utilizing the tetrazolium salt XTT. Journal of immunological methods 142, 257 (1991).

156. R. A. Alvarez-Puebla, E. Arceo, P. J. Goulet, J. J. Garrido, R. F. Aroca, Role of nanoparticle surface charge in surface-enhanced Raman scattering. The Journal of Physical Chemistry B 109, 3787 (2005). 
157. A. Borges, P. Bourban, J. Månson, A composite hydrogel for the replacement of the nucleus pulposus. (2009).

158. X. Li, Y. He, M. T. Swihart, Surface functionalization of silicon nanoparticles produced by laser-driven pyrolysis of silane followed by HF-HNO3 etching. Langmuir 20, 4720 (2004).

159. L. Zhu, J. Ma, N. Jia, Y. Zhao, H. Shen, Chitosan-coated magnetic nanoparticles as carriers of 5-fluorouracil: preparation, characterization and cytotoxicity studies. Colloids and Surfaces B: Biointerfaces 68, 1 (2009).

160. E. Acosta, Bioavailability of nanoparticles in nutrient and nutraceutical delivery. Current opinion in colloid \& interface science 14, 3 (2009).

161. V. Labhasetwar, C. Song, W. Humphrey, R. Shebuski, R. J. Levy, Arterial uptake of biodegradable nanoparticles: effect of surface modifications. Journal of pharmaceutical sciences 87, 1229 (1998).

162. R. P. Bagwe, L. R. Hilliard, W. Tan, Surface modification of silica nanoparticles to reduce aggregation and nonspecific binding. Langmuir 22, 4357 (2006).

163. D. J. Duncker, R. J. Bache, Regulation of coronary blood flow during exercise. Physiological Reviews 88, 1009 (2008).

164. J. Reeff et al., Characterization and optimization of GMO-based gels with long term release for intraarticular administration. International journal of pharmaceutics, (2013).

165. M. H. Shah, S. V. Biradar, A. R. Paradkar, Spray dried glyceryl monooleatemagnesium trisilicate dry powder as cubic phase precursor. International journal of pharmaceutics 323, 18 (2006).

166. A. T. Barker, R. Jalinous, I. L. Freeston, Non-invasive magnetic stimulation of human motor cortex. The Lancet 325, 1106 (1985).

167. A. Pascual-Leone, Transcranial magnetic stimulation: studying the brain-behaviour relationship by induction of 'virtual lesions'. Philosophical Transactions of the Royal Society of London. Series B: Biological Sciences 354, 1229 (1999).

168. M. A. Nitsche et al., Modulation of cortical excitability by weak direct current stimulation-technical, safety and functional aspects. Supplements to Clinical neurophysiology 56, 255 (2003).

169. M. A. Nitsche, W. Paulus, Sustained excitability elevations induced by transcranial DC motor cortex stimulation in humans. Neurology 57, 1899 (2001). 
170. F. Fregni, A. Pascual-Leone, Technology insight: noninvasive brain stimulation in neurology - perspectives on the therapeutic potential of rTMS and tDCS. Nature Clinical Practice Neurology 3, 383 (2007).

171. A. Zangen, Y. Roth, B. Voller, M. Hallett, Transcranial magnetic stimulation of deep brain regions: evidence for efficacy of the H-coil. Clinical Neurophysiology 116, 775 (2005).

172. Y. Tokura, S. Seki, Multiferroics with spiral spin orders. Advanced materials 22, 1554 (2010).

173. M. Fiebig, Revival of the magnetoelectric effect. Journal of Physics D: Applied Physics 38, R123 (2005).

174. V. Parekh et al., Fabrication of a high anisotropy nanoscale patterned magnetic recording medium for data storage applications. Nanotechnology 17, 2079 (2006).

175. S. Khizroev, D. Litvinov, Focused-ion-beam-based rapid prototyping of nanoscale magnetic devices. Nanotechnology 15, R7 (2004).

176. R. Q. So, A. R. Kent, W. M. Grill, Relative contributions of local cell and passing fiber activation and silencing to changes in thalamic fidelity during deep brain stimulation and lesioning: a computational modeling study. Journal of computational neuroscience 32, 499 (2012).

177. L. Abelmann et al., Micromagnetic simulation of an ultrasmall single-pole perpendicular write head. Journal of Applied Physics 87, 6636 (2000).

178. S. Assaf, S.-H. Chung, Release of endogenous $\mathrm{Zn} 2 \&$ plus; from brain tissue during activity. (1984).

179. N. S. Satarkar, J. Z. Hilt, Magnetic hydrogel nanocomposites for remote controlled pulsatile drug release. Journal of Controlled Release 130, 246 (2008).

180. C.-C. Hsiao, P.-C. Chen, H.-B. Pan, J.-C. Jao, Optimal Concentrations of Gadovist in T1-Weighted Magnetic Resonance Imaging: Phantom Study and Computer Simulation. Biomedical Engineering: Applications, Basis and Communications 23, 237 (2011).

181. G. Stuart, N. Spruston, B. Sakmann, M. Häusser, Action potential initiation and backpropagation in neurons of the mammalian CNS. Trends in neurosciences $\mathbf{2 0}$, 125 (1997).

182. M. Mikhaylova et al., BSA immobilization on amine-functionalized superparamagnetic iron oxide nanoparticles. Chemistry of materials 16, 2344 (2004). 
183. A. O. Ivanov et al., Magnetic properties of polydisperse ferrofluids: A critical comparison between experiment, theory, and computer simulation. Physical Review E 75, 061405 (2007).

184. S. Pieper, M. Halle, R. Kikinis, in Biomedical Imaging: Nano to Macro, 2004. IEEE International Symposium on. (IEEE, 2004), pp. 632-635.

185. F. Tadel, S. Baillet, J. C. Mosher, D. Pantazis, R. M. Leahy, Brainstorm: a userfriendly application for MEG/EEG analysis. Computational intelligence and neuroscience 2011, 8 (2011).

186. C. Nicholson, Diffusion and related transport mechanisms in brain tissue. Reports on progress in Physics 64, 815 (2001).

187. C. Wolters et al., Influence of tissue conductivity anisotropy on EEG/MEG field and return current computation in a realistic head model: a simulation and visualization study using high-resolution finite element modeling. NeuroImage 30, 813 (2006).

188. A. Tottene et al., Familial hemiplegic migraine mutations increase $\mathrm{Ca} 2+$ influx through single human CaV2. 1 channels and decrease maximal CaV2. 1 current density in neurons. Proceedings of the National Academy of Sciences 99, 13284 (2002).

189. D. T. Liley, D. M. Alexander, J. J. Wright, M. D. Aldous, Alpha rhythm emerges from large-scale networks of realistically coupled multicompartmental model cortical neurons. Network: Computation in Neural Systems 10, 79 (1999).

190. V. Badilita et al., On-chip three dimensional microcoils for MRI at the microscale. Lab on a Chip 10, 1387 (2010).

191. D. S. Strick et al., in Engineering in Medicine and Biology Society, 2008. EMBS 2008. 30th Annual International Conference of the IEEE. (IEEE, 2008), pp. 2047-2050. 


\section{VITA}

\section{RAKESH GUDURU}

Bachelor of Technology, Biotechnology, 2004-2008, Jawaharlal Nehru Technological University, India

Master of Science, Biomedical Engineering, 2009-2011, Florida International University, Miami, FL

Ph.D. Candidate, Electrical and Computer Engineering, 2011-present, (GPA 3.67/4.00)

Florida International University, Miami, FL (Expected Graduation: December 2013)

\section{PUBLICATIONS AND PRSENTATIONS}

1. Yue, Kun, Rakesh Guduru, Jeongmin Hong, Ping Liang, Madhavan Nair, and Sakhrat Khizroev. "Magneto-Electric Nano-Particles for Non-Invasive Brain Stimulation." PloS one 7, no. 9 (2012): e44040.

2. E. Stefanescu, J. Hong, R. Guduru, A. Lavrenov, D. Litvinov, S. Khizroev. "Magneto-resistive Nanojunctions Fabricated Via Focused Ion Beam Implantation," J. Nanoparticle Research 15 (1), 1387-1390 (2013).

3. Katsenovich, Yelena, Denny Carvajal, Rakesh Guduru, Leonel Lagos, and Chen-Zhong Li. "Assessment of the Resistance to Uranium (VI) Exposure by Arthrobacter sp. Isolated from Hanford Site Soil." Geomicrobiology Journal 30, no. 2 (2013): 120-130.

4. Madhavan Nair, Rakesh Guduru, Ping Liang, Jeongmin Hong, Vidya Sagar, \& Sakhrat Khizroev, (2013). Externally controlled on-demand release of anti-HIV drug using magneto-electric nanoparticles as carriers. Nature communications, 4 , 1707.

5. Vidya Sagar, SK Pilakka-Kanthikeel, H Priestap, VSR Atluri, H Ding, R Guduru, S Khizroev, MP Nair. "Magnetic-nano Formulation of M-opioid Receptor Antagonist (ctop) for Treatment of Morphine-induced Neuropathogenesis in HIV Infection" Journal of Neuroimmune Pharmacology 8 (2), 432-433 (2013).

6. S Khizroev, K Yue, R Guduru, P Liang, J Hong, M Nair. "Magneto-electric Nanoparticles for Non-invasive Brain Stimulation". Journal of Neuroimmune Pharmacology 8 (2), 388-389 (2013). 
7. H Ding, M Agudelo, SP Kanthikeel, R Guduru, V Sagar, V Atluri, S Thangavel, MN Nair. "Dual Mechanism Enhanced BBB Crossing by Transferrin Conjugated Fluorescent Magnetic Liposome". Journal of Neuroimmune Pharmacology 8 (2), 414-414 (2013).

8. Rakesh Guduru, John Bryant, Jeongmin Hong, Ping Liang, Sakhrat Khizroev. "Transport and Photo-voltaic Properties of Bacteriorhodopsin Thin Films for Next-generation Protein Electronics" - MRS conference (2012), San Francisco, CA, April 2012.

9. Rakesh Guduru, Madhavan Nair, Ping Liang, Jeongmin Hong, and Sakhrat Khizroev, "On-Demand Release of Therapeutic Drug Using Magneto-Electric Nanoparticles as a Drug Delivery Vehicle": Submitted to Journal of Visualized Experiments (Invited paper, accepted July 2013. Reference \# 50777).

10. Guduru, Rakesh, Ping Liang, Carolyn Runowicz, Madhavan Nair, Venkata Atluri, and Sakhrat Khizroev. "Magneto-electric Nanoparticles to Enable Fieldcontrolled High-Specificity Drug Delivery to Eradicate Ovarian Cancer Cells." Scientific reports 32953 (2013).

11. Rakesh Guduru and Sakhrat Khizroev, "Magnetic Field-controlled Release of Paclitaxel Drug from Functionalized Magneto-Electric Nanoparticles" Particle (accepted, October 2013. Reference \# ppsc201300238).

12. Rakesh Guduru and Sakhrat Khizroev, "Magneto-Electric Particles for Mapping Electrical Conductivity of brain" Nature Scientific Reports (manuscript in preparation). 